\title{
COMPUTATIONAL MODELING OF ELECTRICAL AND PHONON PROPERTIES OF SKUTTERUDITES AND TWO-DIMENSIONAL TRANSITION METAL DICHALCOGENIDES
}

\author{
by \\ Izaak Gene Williamson
}

\author{
A dissertation \\ submitted in partial fulfillment \\ of the requirements for the degree of \\ Doctorate of Philosophy in Materials Science and Engineering \\ Boise State University
}

May 2017 
(C) 2017

Izaak Gene Williamson

ALL RIGHTS RESERVED 


\title{
BOISE STATE UNIVERSITY GRADUATE COLLEGE
}

\section{DEFENSE COMMITTEE AND FINAL READING APPROVALS}

\author{
of the dissertation submitted by
}

Izaak Gene Williamson

Dissertation Title: Computational Modeling of Electrical and Phonon Properties of Skutterudites and Two-Dimensional Transition Metal Dichalcogenides

Date of Final Oral Examination: $\quad 24$ March 2017

The following individuals read and discussed the dissertation submitted by student Izaak Gene Williamson, and they evaluated his presentation and response to questions during the final oral examination. They found that the student passed the final oral examination.

Lan Li, Ph.D.

Rick Ubic, Ph.D.

Winnie Wong-Ng, Ph.D.

Eric Jankowski, Ph.D.

David Estrada, Ph.D.
Co-Chair, Supervisory Committee

Co-Chair, Supervisory Committee

Member, Supervisory Committee

Member, Supervisory Committee

Member, Supervisory Committee

The final reading approval of the dissertation was granted by Lan Li, Ph.D. and Rick Ubic, Ph.D., Co-Chairs of the Supervisory Committee. The dissertation was approved by the Graduate College. 


\section{DEDICATION}

This dissertation is dedicated to my wife and children. Delanie, you have been a constant companion for me throughout my entire career in higher education. Our love has inspired, encouraged, and supported me through every obstacle, test, setback, success, and achievement. Without you, I would not have come this far. You have shown exceptional strength and patience by taking care of our family whenever I travelled and through late nights, long weekends, and impending deadlines. Kyah and Tayson, I love you more than you will ever realize. Your joy and laughter keeps me smiling, your wonder keeps me motivated, your struggles keep me grounded, and your hearts keep me inspired. The world would be a brighter place if we could all love as you do. 


\section{ACKNOWLEDGEMENTS}

Many factors have contributed to the progress and success I have experienced during my educational career. I am thankful to Boise State University, the Micron School of Materials Science and Engineering, and the Idaho National Laboratory (INL) for their facilities, programs, and computational resources. Also, I have had exceptional support from family, friends, teachers, professors, advisors, collaborators, and classmates. This dissertation would not have been possible without a great deal of help, so I am exceedingly grateful for all the encouragement, assistance, and advice.

First, I would like to acknowledge my advisor, Dr. Lan Li. She has been instrumental for the inception of this dissertation by providing the foundation for my quantum mechanics and DFT knowledge and introducing me to the topics presented in this work. With her assistance and support, I have garnered a number of travel, presentation, collaboration, and internship opportunities which have been crucial for my personal development and the development of this work. Dr. Li remains driven and dedicated to my success for which I will always be grateful.

Next, I would like to thank the members of my committee for their time, support, and suggestions over the past few years. Dr. Rick Ubic has been a large factor in my education, including contributions on: crystallography and microscopy courses, advising my undergraduate senior project, and working with him as editorial assistant for the Materials Research Bulletin (MRB). Dr. Winnie Wong-Ng at the National Institute of Standards and Technology (NIST) has collaborated with our group on numerous projects 
and opened the door for me to research in the fields of energy and thermoelectrics. I am grateful to Drs. Dave Estrada and Eric Jankowski for many helpful discussions and advice in both research and in planning for my future.

Next, I would like to acknowledge the numerous collaborators, internship hosts, and group members that have made this dissertation possible. Dr. Eric Whiting at INL, Dr. Jin Zhao at the University of Science and Technology of China (USTC), Dr. Eric Cockayne at NIST, Dr. Zhun-Yong Ong at the Institute of High Performance Computing in Singapore, Shasha Li and Dr. Yue Chen at the University of Hong Kong, Eric Nelson, Andres Correa Hernandez, Tim Hachigian, Matthew Lawson, Thiago Henrique da Silva, Ember Sikorski, and Kevin Tolman have acted as advisors, colleagues, and friends. I also want to acknowledge the support staff that are the backbone of this research including: Jason Cook, Maureen Moore, Tyler Bevan, Ken Blair, Cormac Garvey, Ben Nickell, Jane Hokanson, Jessica Economy, and Jamie Hayward. The work in this dissertation was partially supported by the NSF EAPSI Fellowship Grant No. 1414593 and computing facilities were provided by Boise State University's R1 cluster and INL's high performance computing (HPC) center.

Finally, I am vastly grateful to my family and friends for the support, encouragement, and reprieve they provide for me. My wife and children uplift, humor, and motivate me every day. It is for them that I am driven to complete this work. My parents, Kali Sherrill and Brad Williamson, have taught me the importance of work ethic, responsibility, respect, critical thinking, and compassion. Growing up, I was pushed, inspired, and cheered by my grandfather, David VanLeeuwen, whom we lost this year. Without the help of him and the rest of my family, I would not be where I am today. 


\begin{abstract}
This dissertation documents the computational modeling of skutterudite and twodimensional transition metal dichalcogenide (2D TMD) materials for energy and electronic applications by analyzing the effects of materials doping and heterostructure formation on structural, energetic, electrical, phonon, and thermal properties. These topics remain largely unexplored and can accelerate materials development by providing insight on structure-properties-performance relationships.
\end{abstract}

Skutterudites are commonly studied for thermoelectric applications because they are low-cost, easy to process, and offer good intrinsic transport properties. They also exist as large, open structures which can be altered through filler atoms or substitutional dopants. A density functional theory (DFT)-based investigation of dopant effects on skutterudite compounds provided insight to advance the understanding of electrical and phonon properties that experiments could not measure. This also offered a good benchmark material for developing a modeling scheme that was employed for 2D TMD materials. 2D TMD nanosheets also exhibit large variability in structure type, dimensionality, and composition and have attracted much interest for their magnetic, electronic, optoelectronic, catalytic, and thermoelectric properties. Their low dimensionality makes them promising candidates for field-effect transistor (FET) device applications and introduces quantum confinement effects and diffusive boundary scattering, potentially improving their electrical and transport properties. The exploration 
of composition, substitutional doping, and heterostructure effects is needed for further 2D TMD materials development and property improvement.

This dissertation offers an analysis of the structure-property relationships for a wide range of properties on bulk skutterudite and 2D TMD materials. The three key outcomes of this work are: (1) a high throughput approach to compute and analyze electrical and phonon properties, (2) a screening method for investigating 2D TMD materials and highlighting preferred compositions, and (3) design principles for predicting structures and properties to guide experiments. The optimized high throughput approach encompasses: DFT-based total energy minimization calculations to investigate the geometric, energetic, and electronic structure data; Boltzmann transport theory, in combination with electronic band energies, to estimate electrical conductivity $(\sigma)$, Seebeck coefficient $(S)$, and power factor $\left(S^{2} \sigma\right)$ values; density functional perturbation theory (DFPT)-based second-order force constant calculations to determine phonon dispersion and density of states (DoS) spectra; and the atomistic Green's function (AGF) method, using force constants as input, to compute interfacial heat flux, phonon transmission coefficients, and thermal boundary conductance (TBC). Error mitigation was handled by optimizing model parameters and validating results through comparison with literature and experimental data. Through the optimized high throughput approach, dozens of 2D TMD structures can now be analyzed within days, whereas initial optimization calculations for each structure took up to one week to compute. Overall, these materials offer great potential for materials-by-design exploration and understanding their structural, electrical, and phonon properties are essential for advancement towards commercial applications. 
TABLE OF CONTENTS

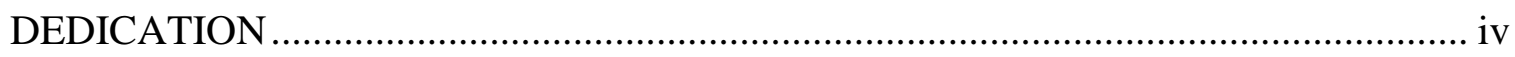

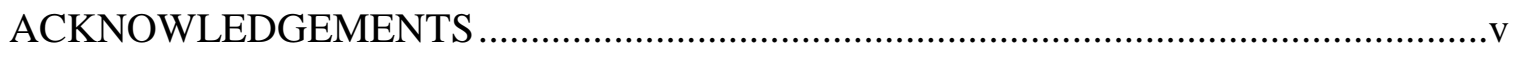

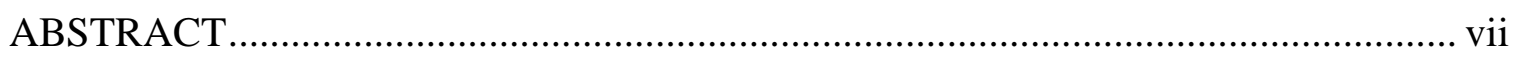

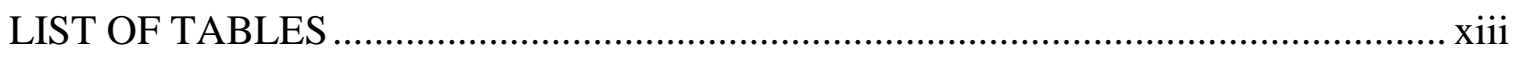

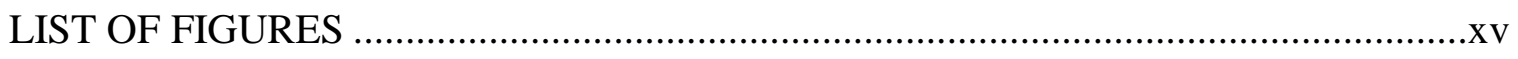

LIST OF ABBREVIATIONS ............................................................................

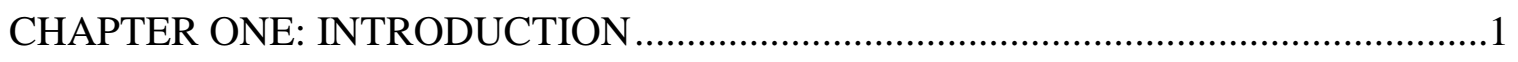

1.1 Research Goal and Motivation...................................................................

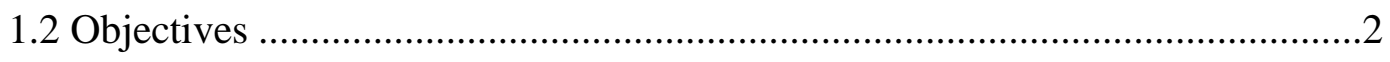

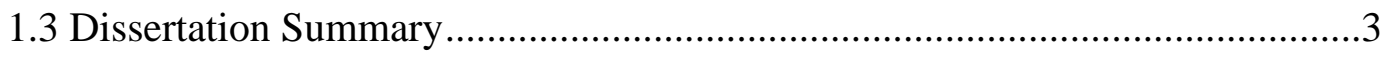

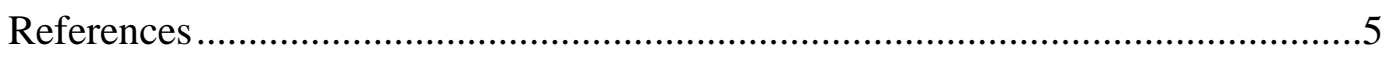

CHAPTER TWO: MATERIALS SYSTEMS …………...........................................

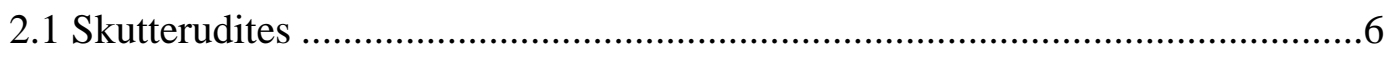

Current State of Skutterudite Materials Research.......................................8

2.2 Two-Dimensional Transition Metal Dichalcogenides (2D TMDs) ...................9

Current State of TMD Materials Research ……………...........................12

2.3 Computational Modeling Approaches …………….........................................13

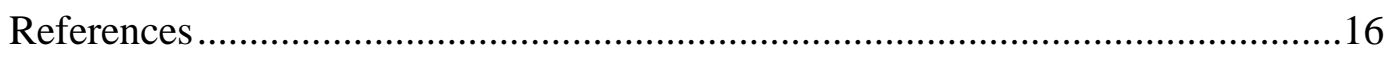

CHAPTER THREE: MODELING ELECTRICAL AND THERMAL PROPERTIES IN

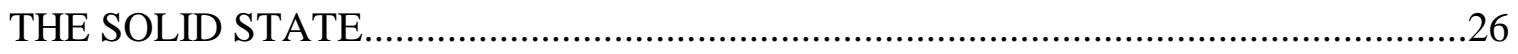




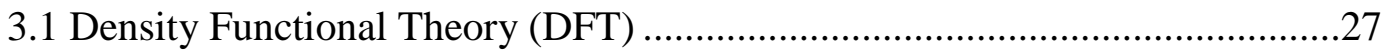

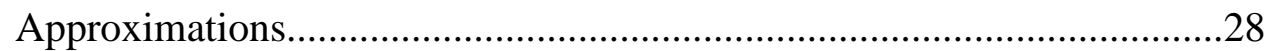

3.2 Electrical Transport Properties ..............................................................34

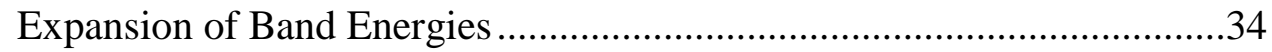

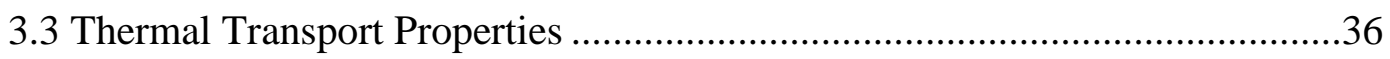

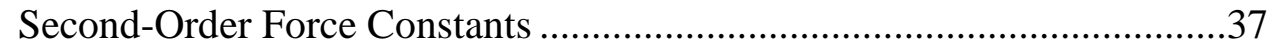

Atomistic Green's Function (AGF) Method..........................................38

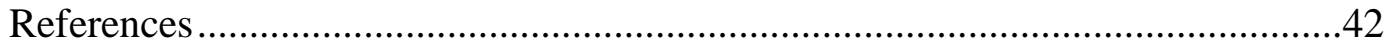

CHAPTER FOUR: IMPROVED THERMOELECTRIC PERFORMANCE OF (Fe,Co)Sb3-TYPE SKUTTERUDITES FROM FIRST-PRINCIPLES* ........................45

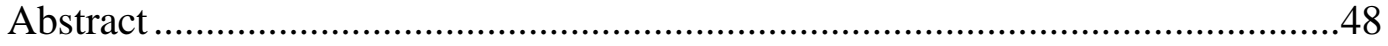

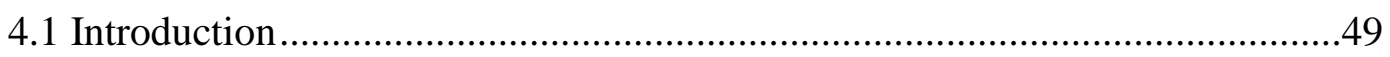

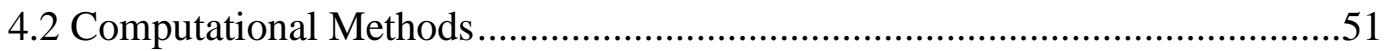

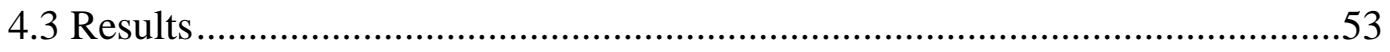

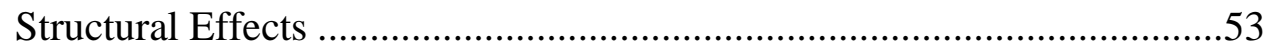

Electrical Properties ....................................................................55

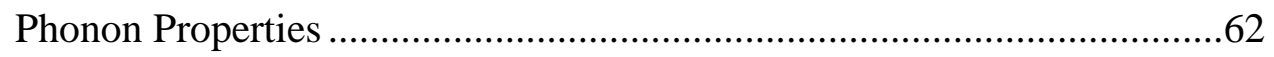

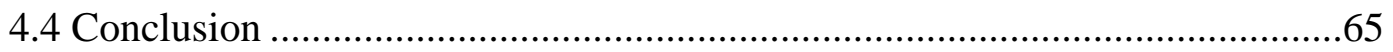

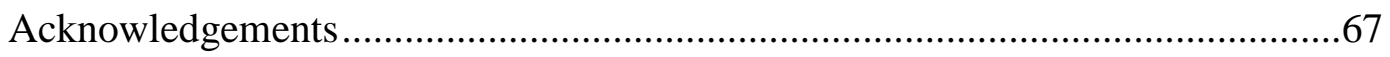

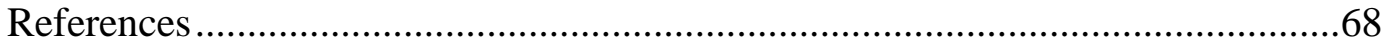

CHAPTER FIVE: HIGH-THROUGHPUT COMPUTATIONAL SCREENING OF ELECTRICAL AND PHONON PROPERTIES OF TWO-DIMENSIONAL TRANSITION METAL DICHALCOGENIDES*

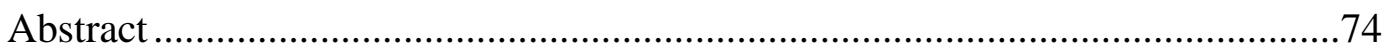

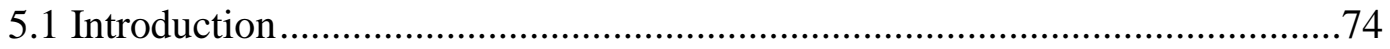


5.2 Computational Methods

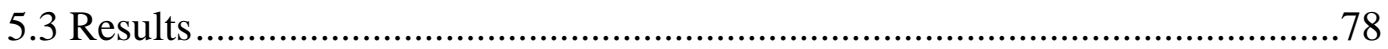

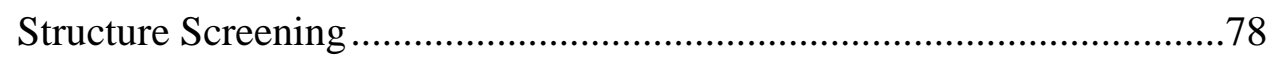

Electrical Property Screening ……………………………………….... 81

Phonon Property Screening........................................................................8

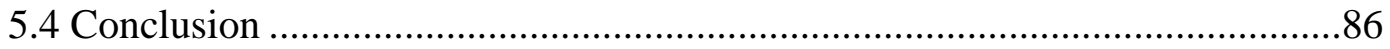

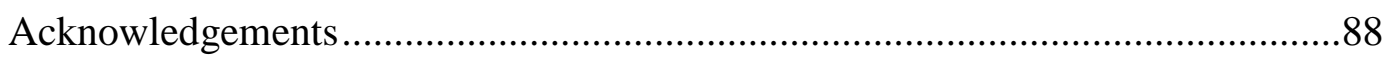

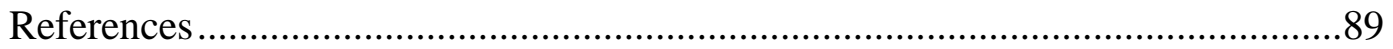

CHAPTER SIX: IMPROVEMENT OF 2D TMD MATERIALS THROUGH METALSITE SUBSTITUTIONAL DOPANTS*...................................................................94

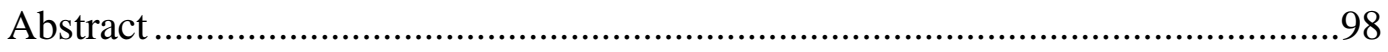

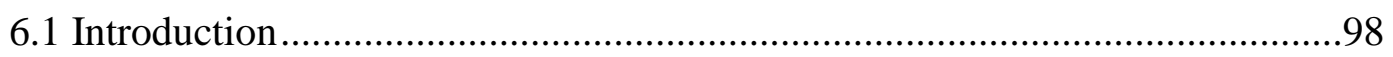

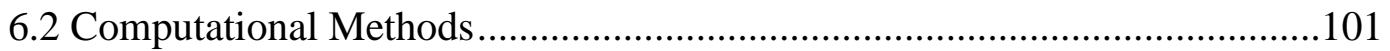

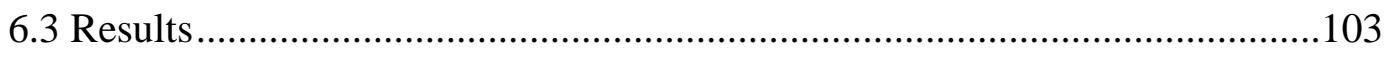

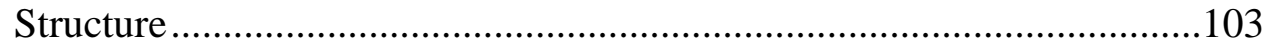

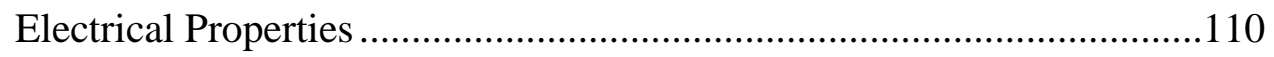

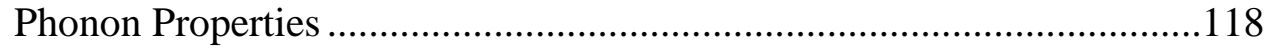

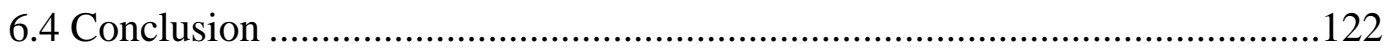

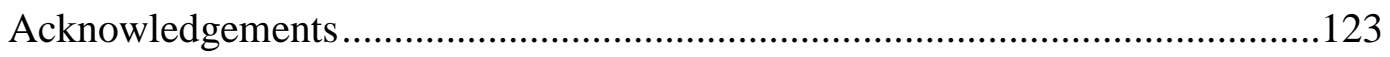

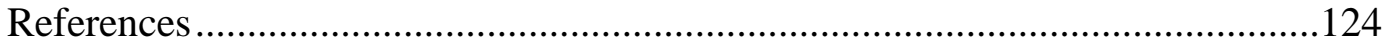

CHAPTER SEVEN: HETEROSTRUCTURE EFFECTS ON STRUCTURAL, ELECTRICAL, AND PHONON PROPERTIES OF TWO-DIMENSIONAL TRANSITION METAL DICHALCOGENIDES

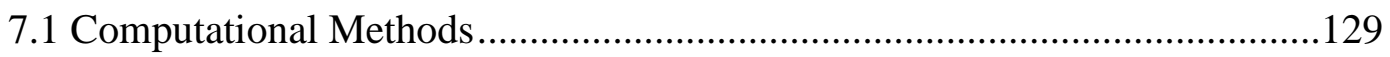

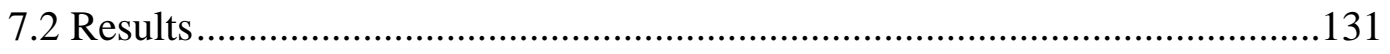




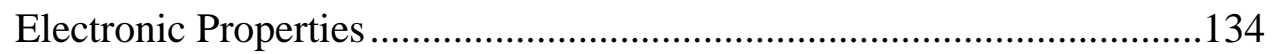

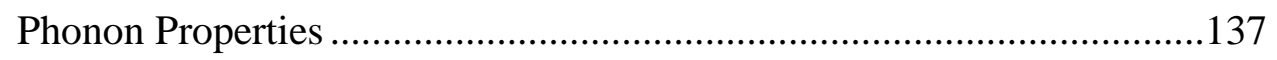

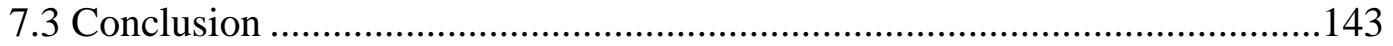

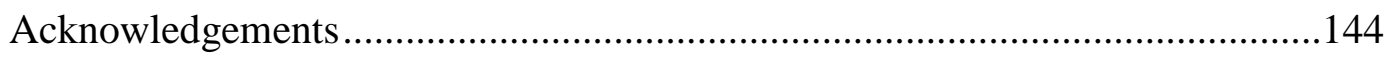

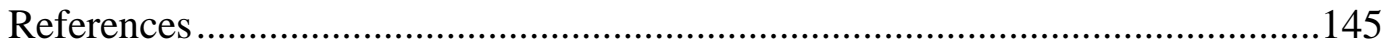

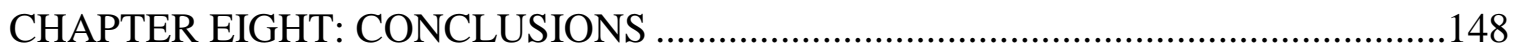




\section{LIST OF TABLES}

Table 4.1 Calculated cell angle $\gamma$ in degrees and lattice parameter $a$ in units of $\AA$ for $\mathrm{Co}_{4} \mathrm{Sb}_{12-2 \mathrm{x}} \mathrm{Te}_{\mathrm{x}} \mathrm{Ge}_{\mathrm{x}}(x=0,0.5,1,2,3$, and 6). The experimentally determined lattice parameter $a_{\text {exp }}$ and the percent error between calculated and experimental values is also given (subscript $\exp _{\text {denotes experimental }}$ values). ${ }^{31}$ The symbol $\theta$ represents the smallest distortion angle in the pnictogen rings. Superscript ${ }^{a}$ denotes values taken for the structure with the highest dopant site order (configuration 3).

Table 5.1 Oxidation state, atomic mass $(\mathrm{u})$, and atomic radius $(\AA)$ for each transition metal. The 2D TMDs $\left(\mathrm{MX}_{2}\right)$, consisting of a transition metal $(M)$ and chalcogen $(X)$, have either $2 \mathrm{H}$ - or 1T-type structures. Lattice parameter $a$ $(\AA)$, layer thickness $t(\AA)$, and electronic bandgap $E_{g}(\mathrm{eV})$ of the corresponding TMD are also given. ${ }^{\text {a}}$ Reference. ${ }^{41}$ beference. ${ }^{42}$ Values taken from list of crystal radii with +4 charge and six-fold coordination. ${ }^{\mathrm{c}}$ Value for +3 charge. ${ }^{\mathrm{d}} E_{F}$ sits on mid-gap state.

Table 5.2 Power factor $\left(S^{2} \sigma\right)$ values for the 18 2D TMDs. Values are reported as $10^{3}$ times larger than actual for the purpose of clarity in the table. Each value, when multiplied by $10^{-3}$, yields units of $\mathrm{W} / \mathrm{m} / \mathrm{K}^{2}$. These are calculated at $300 \mathrm{~K}$ for both $n$ - and $p$-type

Table 6.1. Geometric structure results for pure and $\mathrm{M}^{\prime}$-doped $\left(\mathrm{M}^{\prime}=\mathrm{Ti}\right) 2 \mathrm{D} \mathrm{MoS}_{2}$. The average lattice parameter with respect to the original unit cell, $a_{u c}$, the M-S and S-S bond distances, $d_{M-S}$ and $d_{S-S}, \mathrm{~S}-\mathrm{M}-\mathrm{S}$ bond angles, $\theta$, and binding energies, $E_{b}$, are given. All distances are given in angstroms $(\AA)$ and all angles are given in degrees $\left(^{\circ}\right)$. Superscripts ${ }^{M}$ and ${ }^{M o}$ indicate values taken at the dopant site and at the Mo site farthest from the defect, respectively. 105

Table 6.2. Structure properties of metal-site doped $\mathrm{MX}_{2}$ (having the formula $\mathrm{M}^{\prime} \mathrm{M}_{15} \mathrm{X}_{32}$ where $\mathrm{M}^{\prime}=$ dopant atom Mo, Ni, Sc, Ti, V, or W; M = Mo or $\mathrm{W}$; and $\mathrm{X}=\mathrm{S}$, Se, or Te) compared to the undoped values. The average lattice parameter per unit cell ( $4 \mathrm{x} 4$ unit cells per structure), $\mathrm{a}_{\mathrm{uc}}, \mathrm{M}^{\prime}-\mathrm{X}$ bond length, $\mathrm{X}-\mathrm{X}$ bond length, and $\mathrm{X}-\mathrm{M}-\mathrm{X}$ bond angle are given in units of either angstroms $(\AA)$ or degrees $\left({ }^{\circ}\right)$. The percent difference from the corresponding undoped value is listed as $\Delta \%$ 
Table 7.1. Physical features of the transition metals, $\mathrm{M}$, in $2 \mathrm{D} \mathrm{MoS}_{2}-\mathrm{MS}_{2}$ lateral heterostructures. The lattice, $\Delta a$, and S-S distance, $\Delta d_{s-S}$, mismatches are based off of the pure $2 \mathrm{D} \mathrm{MoS} 2$ and $\mathrm{MS}_{2}$ structures. The formation energy and electronic bandgap of the heterostructure is given as $E_{f}$ and $E_{g}$, respectively. 


\section{LIST OF FIGURES}

Figure 2.1. Crystal structure of a filled $\mathrm{M}_{2} \mathrm{~A}_{8} \mathrm{~B}_{24}$ skutterudite with filler atom $\mathrm{M}$ in red, $\mathrm{B}$ in white, and A in black. The gray substructures outlined by $\mathrm{B}$ atoms are referred to as pnictogen rings.

Figure 2.2. Top-down view of 2D TMD sheets having 2H- or 1T-type structures. Cyan and yellow spheres represent $\mathrm{M}$ and $\mathrm{X}$ atoms, respectively. Thin black lines frame the unit cell. Armchair and zigzag directions are depicted with bold black arrows.

Figure 3.1. Flowchart describing the computational approach used to calculate electrical and thermal transport properties in this dissertation. DFT is used to minimize the total energy of the system in order to determine the electronic ground state. This can then be used to calculate structural features, band energies, and DFPT force constants. The band energies can be expanded to calculate electrical transport coefficients such as $S, \sigma$, and $\kappa_{\mathrm{e}}$. The second-order force constants can be used to calculate phonon DoS or combined with the AGF method to determine phonon transmission coefficients across a heterojunction.

Figure 3.2. Schematic demonstrating the pseudo-electron (PE) (dotted lines) and allelectron (AE) (solid lines) wave function, $\Psi$, and potential, V. The PE and AE values must match at $r \geq r_{c}$ where $r_{c}$ represents the core radius. 31

Figure 3.3. Flowchart describing the scf and structural relaxation method performed during a DFT calculation. A single scf step is completed when the first "convergence" step is reached. By calculating the forces after an scf step and adjusting the atomic positions, the forces can be minimized until a "relaxed" ground state configuration is determined.

Figure 4.1. Schematic of the $\mathrm{Ca}_{4} \mathrm{Ce}_{4} \mathrm{Fe}_{32} \mathrm{Sb}_{96}$ crystal structure where $\mathrm{Ca}$ (blue), Ce (green), Fe (black), and $\mathrm{Sb}$ (gray) atoms are represented by spheres. The preferred ordering of the filler atoms is to arrange all $\mathrm{Ca}$ atoms along (220) planes and all Ce atoms along (110) planes in this 4x4x2 supercell.

Figure 4.2. Model depicting pnictogen rings within the filled skutterudite structure. Upon Te/Ge substitution, the ring distorts to an angle of down to $81^{\circ}$ for $\mathrm{Co}_{4} \mathrm{Sb}_{12-2 \mathrm{x}} \mathrm{Te}_{\mathrm{x}} \mathrm{Ge}_{\mathrm{x}}$ at $x=3$. 
Figure 4.3. Electronic density of states for the unfilled (black line) and CaCe-filled (red line) $\mathrm{FeSb}_{3}$ structure. In order to account for the correct $\mathrm{Ca}-\mathrm{Ce}$ ordering as shown in Figure 4.1, a large $\mathrm{Ca}_{4} \mathrm{Ce}_{4} \mathrm{Fe}_{32} \mathrm{Sb}_{96}$ supercell structure was used. For the sake of comparison, the DoS magnitude for the unfilled structure is increased by a factor of four. Dotted lines depict the zero values on both axes and the $\mathrm{x}$-axis is shifted so the zero-point represents the Fermi level (depicted by the blue dotted line). The up- and down-spin DoS are given by positive and negative values, respectively. The difference in shape between spin-up and spin-down is primarily caused by the magnetism of the Fe atoms in the system. The unfilled $\mathrm{Fe}_{8} \mathrm{Sb}_{24}$ (black) results show a bandgap only for the positive DoS while neither the up- nor down-spin CaCe-filled (red) DoS has a significant bandgap

Figure 4.4. Electronic density of states for $\mathrm{Co}_{4} \mathrm{Sb}_{12-2 \mathrm{x}} \mathrm{Te}_{\mathrm{x}} \mathrm{Ge}_{\mathrm{x}}$, Fermi energy $=0 \mathrm{eV}$ : (a) Total DoS for $x=0,0.5,1,2$, and 3; and (b) local DoS (LDoS) for $\mathrm{Co}_{8} \mathrm{Sb}_{22}$ (black), Te (red), and Ge (blue) in $\mathrm{Co}_{8} \mathrm{Sb}_{22} \mathrm{TeGe}$ (i.e., $\mathrm{Co}_{4} \mathrm{Sb}_{11} \mathrm{Te}_{0.5} \mathrm{Ge}_{0.5}$ with $x=0.5$ ). DoS results in (a) indicate that the bandgap decreases with increasing $x$. Part (b) shows an interaction between the local Te and Ge densities indicating orbital hybridization... 58

Figure 4.5. Electronic band structure diagrams of $\mathrm{Co}_{4} \mathrm{Sb}_{12-2 \mathrm{x}} \mathrm{Te}_{\mathrm{x}} \mathrm{Ge}_{\mathrm{x}}$ for (a) $x=0$, (b) $x$ $=0.5$, and (c) $x=3$. Blue arrows indicate the changes in the direct and indirect bandgaps. As substitution increases, the bandgap decreases from (a) $0.16 \mathrm{eV}$ to (b) $0.13 \mathrm{eV}$, and eventually to (c) $0.11 \mathrm{eV}$ for the highestdoped structure investigated $(x=3)$. Also note the transition from direct to indirect bandgap semiconducting behavior in (c). 60

Figure 4.6. Density of states plots for $\mathrm{Co}_{4} \mathrm{Sb}_{12-2 \mathrm{x}} \mathrm{Te}_{\mathrm{x}} \mathrm{Ge}_{\mathrm{x}}$ where (a) $x=3$ and (b) $x=6$. Fermi energy is zero. The black, green, and blue plots depict the randomly ordered (configuration 1), the partially ordered (configuration 2), and fully ordered (configuration 3) pnictogen ring configurations. The pnictogen ring ordering increasingly opens up the bandgap. An example of each pnictogen ring configuration (depicted using the $x=3$ structure) is shown in part (c) where the blue line shows Co-Co bonds and the gray, yellow, and red spheres depict $\mathrm{Sb}, \mathrm{Te}$, and Ge atoms, respectively.

Figure 4.7. Phonon density of states for unfilled $\mathrm{Fe}_{8} \mathrm{Sb}_{24}$ (black) and CaCe-filled $\mathrm{Ca}_{4} \mathrm{Ce}_{4} \mathrm{Fe}_{32} \mathrm{Sb}_{96}$ (blue). To account for the proper $\mathrm{Ca} / \mathrm{Ce}$ filler atom ordering which provides the most stable structure (and most promising thermoelectric performance) of the $\mathrm{Ca}_{0.5} \mathrm{Ce}_{0.5} \mathrm{Fe}_{4} \mathrm{Sb}_{12}$ system, the larger 136-atom supercell was used for the "CaCe-filled" structure.

Figure 4.8. Phonon property calculations. (a) Phonon dispersion relation for $\mathrm{Co}_{4} \mathrm{Sb}_{12-}$ ${ }_{2 \mathrm{x}} \mathrm{Te}_{\mathrm{x}} \mathrm{Ge}_{\mathrm{x}}$ at $x=0$. Similar plots were made for $x=3$ and $x=6$ but for brevity, only their acoustic modes (along with those of $x=0$ ) are given in 
(b), and (c) total phonon density of states for each compound, projected across the full range of mode frequencies.

Figure 5.1. Effects of (a) atomic mass and (b) atomic radius of the transition metal on the lattice constant, $a$, and layer thickness of the resulting 2D TMD structure. Circles and diamonds refer to lattice constant and layer thickness values, respectively. Open and filled data points refer to 1T- and $2 \mathrm{H}$-type $\mathrm{MX}_{2}$ structures, respectively. Solid and dashed linear trend lines have been added to plot (b) to guide the eye for lattice constant and layer thickness data, respectively.

Figure 5.2. Seebeck coefficient, $S(\mu \mathrm{V} / \mathrm{K})$, calculated as a function of carrier concentration $N\left(\mathrm{e} / \mathrm{cm}^{2}\right)$ for $2 \mathrm{D}$ TMDs. Plots (a)-(c) show the $p$-type $S$ values while plots (d)-(f) depict $n$-type values. The first (a,d), second (b, e), and third (c, f) columns compare the $\mathrm{MS}_{2}, \mathrm{MSe}_{2}$, and $\mathrm{MTe}_{2}$ systems, respectively. The $\mathrm{MoX}_{2}, \mathrm{TiX}_{2}$, and $\mathrm{WX}_{2}$ systems all exhibit semiconducting behavior and are distinguished by lines with diamonds (Mo diamonds-solid line, Ti diamonds-dashed line, and $\mathrm{W}$ diamonds-dotdashed line). The more-conductive $\mathrm{NiX}_{2}, \mathrm{ScX}_{2}$, and $\mathrm{VX}_{2}$ systems all exhibit low $S$ values and are depicted using lines (Ni dotted line, Sc dotdot-dashed line, and V dash-dash-dotted line). All calculations were performed at the temperature of $300 \mathrm{~K}$.

Figure 5.3. Projected phonon DoS for each member of the $\mathrm{MS}_{2}$ system. Each plot depicts the total phonon DoS with a solid line, the contribution from the transition metal with a dot-dashed line, and the contribution from the $\mathrm{S}$ atoms with a dashed line. Plots (a)-(f) are shown in order of increasing atomic mass of the transition metal as follows (a) $\mathrm{ScS}_{2}$, (b) $\mathrm{TiS}_{2}$, (c) $\mathrm{VS}_{2}$, (d) $\mathrm{NiS}_{2}$, (e) $\mathrm{MoS}_{2}$, and (f) $\mathrm{WS}_{2}$

Figure 6.1. Top-down view of $\mathrm{M}^{\prime}$-doped $\mathrm{MX}_{2}$ where the $\mathrm{M}^{\prime}$ dopant atom is depicted with a black sphere, the $\mathrm{M}$ atoms are gray spheres, and the $\mathrm{X}$ chalcogens are yellow spheres.

Figure 6.2. Schematic of an $\mathrm{MS}_{2}$ octahedron where the violet and yellow spheres represent $\mathrm{M}$ and $\mathrm{S}$ atoms, respectively. In this work, the $\mathrm{M}$ atom depicts either the dopant, M' (Ti or V), or the constituent, Mo. The bond lengths are represented by red arrows where $\mathrm{d}_{\mathrm{S}-\mathrm{S}}$ and $\mathrm{d}_{\mathrm{M}-\mathrm{S}}$ depict the distance between two $\mathrm{S}$ atoms and between the $\mathrm{M}$ and $\mathrm{S}$ atoms, respectively. The S-M-S bond angle is represented by the blue lines and the symbol $\theta$... 105

Figure 6.3. Dopant atom binding energy $\left(E_{b}\right)$ for each system listed in order of increasing dopant atomic radius. Solid black, dashed blue, and dotted red lines represent $\mathrm{MS}_{2}, \mathrm{MSe}_{2}$, and $\mathrm{MTe}_{2}$ systems, respectively. Circle-lines depict $\mathrm{MoX}_{2}$ structures while diamond-lines depict $\mathrm{WX}_{2}$ structures. More- 
negative $E_{b}$ values (greater absolute values) correspond to greater stability.

Figure 6.4. Electronic DoS for $2 \mathrm{D} \mathrm{MoS}_{2}$ having increasing atomic percentage concentrations of Ti dopants. These concentrations include 0.926 (blue), 1.333 (red), 2.083 (green), 3.704 (orange), and 8.333 (violet) at.\% Ti. The pure $\mathrm{MoS}_{2}$ data is shown for comparison and depicted with a black line. All data is shifted to align each plot's respective Fermi levels $\left(E_{F}\right.$ 's) to $x=$ 0 (represented by the vertical dashed black line). Inset focuses on the area immediately surrounding $E_{F}$. 111

Figure 6.5. LDoS calculated as a function of electron energy (shifted to $E_{F}=0 \mathrm{eV}$ ) for the $\mathrm{Mo}_{15} \mathrm{M}_{1} \mathrm{~S}_{32}$ system (2.083 at.\% M' where M' = Ti or V). The black line represents pure $\mathrm{MoS}_{2}$ while the blue and red lines depict Ti-doped and $\mathrm{V}$-doped $\mathrm{MoS}_{2}$ systems, respectively. The top plot has the total DoS for each system while the bottom three show local contributions per atom (avg.) from each of the Mo, S, and M' atoms, respectively. The vertical dashed black line indicates $E_{F}$.

Figure 6.6. Electronic DoS for each doped system. Column 1 (a, c, and e) contains the $\mathrm{MoX}_{2}$ data while column 2 (b, d, and f) contains the $\mathrm{WX}_{2}$ data. The rows depict sulfide ( $\mathrm{a}$ and $\mathrm{b}$ ), selenide ( $\mathrm{c}$ and $\mathrm{d}$ ), and telluride (e and f) systems, respectively. Undoped, Ni-, Sc-, Ti-, and V-doped systems are represented by black, blue, red, green, and orange lines, respectively. Both the $\mathrm{W}$ doped systems in column 1 (a, c, and e) and the Mo-doped systems in column 2 (b, d, and f) are shown as violet lines.

Figure 6.7. Boltzmann transport properties calculated as a function of carrier concentration, $N$, for $\mathrm{W}$-doped $\mathrm{MoX}_{2}$ (plots a, c, and e) and Mo-doped $\mathrm{WX}_{2}$ (plots b, d, and f). The Seebeck coefficient, $S$, electrical conductivity, $\sigma / \tau$, and power factor, $S^{2} \sigma / \tau$, are shown in rows (a-b), (c-d), and (e-f), respectively. Sulfide, selenide, and telluride systems are represented by solid black, dashed blue, and dotted red plots, respectively, while diamond-line plots and circle-line plots depict W- and Mo-doped systems, respectively.

Figure 6.8. Phonon DoS calculated for various concentrations of Ti dopants in 2D $\mathrm{MoS}_{2}$. Data is shown normalized per formula unit. The black line represents pure $\mathrm{MoS}_{2}$ with 0 at.\% Ti while the colored lines represent 0.926 (blue), 1.333 (red), 2.083 (green), 3.704 (orange), and 8.333 (violet) at.\% $\mathrm{Ti}$.

Figure 6.9. Total phonon DoS calculated for $\mathrm{MoS}_{2}$ doped with 2.083 at.\% Ti (blue) and 2.083 at.\% V (red). The black plot represents pure $\mathrm{MoS}_{2}$. Data is shown normalized per formula unit. Insert compares these results against 
the partial contributions from the dopant atoms (dashed lines) and $\mathrm{S}$ atoms bonded to the dopants (circles), focusing on the frequency gap region.. 121

Figure 7.1. Schematic of the $\mathrm{MoS}_{2}-\mathrm{MS}_{2}(\mathrm{M}=\mathrm{Cr}, \mathrm{Sc}, \mathrm{Ti}$, or W) heterostructure where the Mo, S, and $\mathrm{M}$ atoms are depicted using gray, yellow, and black spheres, respectively

Figure 7.2. Electronic band structure diagrams for $\mathrm{MoS}_{2}-\mathrm{CrS}_{2}$ and $\mathrm{MoS}_{2}-\mathrm{WS}_{2}$ lateral heterostructures. The $y$-axis for both plots has been shifted to $E_{F}=0 \mathrm{eV}$. The $\mathrm{MoS}_{2}-\mathrm{WS}_{2}$ structure is determined to be a direct semiconductor at the $\Gamma$ point whereas the lowest conduction band in the $\mathrm{MoS}_{2}-\mathrm{CrS}_{2}$ structure has shifted slightly towards the $\mathrm{K}$ point exhibiting indirect semiconducting behavior (as indicated by the blue arrow) ......................................... 135

Figure 7.3. Band alignments for each of the pure 2D $\mathrm{MS}_{2}(\mathrm{M}=\mathrm{Cr}, \mathrm{Mo}, \mathrm{Sc}, \mathrm{Ti}$, and W) structures shifted to the vacuum level. Horizontal black lines represent the Fermi level $\left(E_{F}\right)$ for each structure.

Figure 7.4. Phonon density of states calculated for each 2D $\mathrm{MoS}_{2}-\mathrm{MS}_{2}$ heterostructure where the yellow $(\mathrm{Cr})$, green $(\mathrm{Sc})$, blue $(\mathrm{Ti})$, and black $(\mathrm{W})$ plots represent each respective transition metal, M. Negative (imaginary) phonon frequencies are found for the two least stable (lowest formation energy) heterostructures: $\mathrm{M}=\mathrm{Sc}$ and Ti.

Figure 7.5. Phonon transmission coefficients across the $\mathrm{MoS}_{2}-\mathrm{WS}_{2}$ interface for the ZA ( $a$ and $b$ ), TA ( $c$ and d), and LA (e and f) acoustic phonon modes. The left column (a, c, and e) represents $\mathrm{MoS}_{2}$ phonons contributing to leftward interfacial heat flux whereas the right column (b, d, and f) represents $\mathrm{WS}_{2}$ phonons contributing to rightward interfacial heat flux. 140

Figure 7.6. Thermal boundary conductance (TBC) for $\mathrm{MoS}_{2}-\mathrm{WS}_{2}$ lateral interface. The dotted black plot represents the total TBC for the system and the dotted pink plot represents the TBC contribution from the acoustic phonon modes. 


\section{LIST OF ABBREVIATIONS}

$2 \mathrm{D}$

2D TMD

$3 \mathrm{D}$

$a$

$\AA$

AGF

BoltzTraP

CBM

DFT

$\mathrm{DFT}+\mathrm{U}$

DoS

$\mathrm{d}_{\mathrm{S}-\mathrm{S}}$

$\mathrm{E}_{\text {cut }}$

$\mathrm{E}_{\mathrm{f}}$

$\mathrm{E}_{\mathrm{F}}$

$\mathrm{E}_{\mathrm{g}}$

EMD

$\mathrm{eV}$

FET

GKF
Two-dimensional

Two-dimensional transition metal dichalcogenide

Three-dimensional

Lattice constant

Angström

Atomistic Green's function

Boltzmann transport properties

Conduction band minimum

Density functional theory

Density functional theory with Hubbard U correction

Density of States

Vertical distance between $\mathrm{S}$ chalcogens in $2 \mathrm{D} \mathrm{MS}$ 's

Cutoff energy

Formation energy

Fermi level

Electronic bandgap

Equilibrium molecular dynamics

Electron volts

Field-effect transistor

Green Kubo formula 


$\begin{array}{ll}\text { GGA } & \text { Generalized gradient approximation } \\ h \text {-BN } & \text { Hexagonal boron nitride } \\ \kappa & \text { On-site exchange potential } \\ \text { LDA } & \text { Thermal conductivity } \\ \text { PAW } & \text { Local density approximation } \\ \text { PBE } & \text { Projector augmented wave } \\ \sigma & \text { Perdew-Burke-Ernzerhof } \\ S & \text { Electrical conductivity } \\ S^{2} \sigma & \text { Seebeck coefficient } \\ \tau & \text { Power factor } \\ \text { TBC } & \text { Relaxation time } \\ \text { TMD } & \text { Thermal boundary conductance } \\ \text { TMO } & \text { Transition metal dichalcogenide } \\ \mu \text { V } & \text { Transition metal dioxide } \\ \mathrm{U} & \text { Micro-volts } \\ \text { VASP } & \text { On-site Coulomb potential } \\ \text { VBM } & \text { Vienna } a b \text { initio simulation package } \\ \text { vdW } & \text { Thermoelectric figure of merit } \\ \text { ZT } & \text { vand }\end{array}$




\section{CHAPTER ONE: INTRODUCTION}

\subsection{Research Goal and Motivation}

To accelerate materials development, this dissertation aims to derive basic materials design principles through high-throughput computational screening methods. By generating large and rich data sets and analyzing the data to capture changes in trends and relationships between structure, properties, and performance, we can optimize materials' properties and performance by tailoring their structures.

This dissertation focuses on the area of electronic and energy applications and how they can be directly improved through the implementation and successful completion of this research goal. For the past fifty years, the semiconductor industry, for example, has been striving to maintain growth in line with Moore's Law, which predicts that the number of transistors present in an integrated circuit would double every two years. The industry has managed to keep pace with this prediction through a number of innovations in chip design, photolithography, and processing techniques. Smaller feature sizes approach physical limitations as well as dimensionality constraints. Thus, it is crucial to understand electrical transport behavior in this regime as quantum effects take over.

With reduction in the size of device features comes an increase in energy density within the chips. Joule heating, via the conversion of electrical power to thermal energy, causes internal temperatures to exceed that of safe, operable conditions. This leads to excessive wear, premature device failure, and safety hazards for people operating these 
devices. The issue of understanding electrical and thermal properties is nontrivial and further work is needed to improve such devices.

This work investigates the key parameters that influence electrical and thermal properties. They include the analysis of electron and phonon density of states (DoS) spectra and the calculation of electrical conductivity, $\sigma$, and the Seebeck coefficient, $S$, which is a material's voltage response to a temperature gradient (useful for thermoelectric and thermocouple applications). Materials systems with potential applications in these fields include skutterudites and two-dimensional transition metal dichalcogenides (2D TMDs). These have good intrinsic electrical properties and tailorable structures, making them good candidates for energy and electronic applications, but more work is needed to further improve their properties. To achieve the research goal, the effects of two main structural changes have been investigated: (1) doping and (2) the formation of a heterojunction.

\subsection{Objectives}

This project has four research objectives.

\section{Objective 1: Understand dopant and filler atom effects on skutterudite materials}

(Chapter Four) This includes the analysis of bulk (Fe,Co)Sb3 skutterudites and how they are affected by $\mathrm{Ca} / \mathrm{Ce}$ filler and Te/Ge substitutional dopants. This allows for the analysis of dopant effects on electrical and phonon properties in a three-dimensional material while also acting as a foundation for the development of the computational approach. Objective 2: Computationally screen 2D TMD material compositions (Chapter Five) The physical features of six different constituent metals (Mo, Ni, Sc, Ti, V, and W) are 
compared with respect to three different chalcogenide (S, Se, and Te) compounds while translating the previous computational approach to a lower dimensionality.

Objective 3: Understand the effects of substitutional dopants in 2D TMDs (Chapter Six)

Each of the six transition metals from objective 2 are individually substituted into metalsites within the $2 \mathrm{D} \mathrm{MoX}_{2}$ and $\mathrm{WX}_{2}(\mathrm{X}=\mathrm{S}, \mathrm{Se}$, or Te) structures. Structural, electrical, and phonon properties are analyzed, focusing on the effect of substitution concentration and type.

Objective 4: Evaluate the impact of a lateral heterojunction between 2D TMDs (Chapter $\underline{\text { Seven })}$ The effects of laterally joining $2 \mathrm{D} \mathrm{MoS} 2$ with $2 \mathrm{D} \mathrm{MS} 2(\mathrm{M}=\mathrm{Cr}, \mathrm{Sc}, \mathrm{Ti}$, and W) to form a heterostructure are investigated. Structural, electrical, and phonon effects are compared.

\subsection{Dissertation Summary}

This dissertation includes the investigation of structural, electrical, and phonon properties of 3D skutterudite materials and 2D TMD nanosheets from first-principles. At the time this dissertation is being written, three peer-reviewed papers have been published from this work in the Journal of Applied Physics, JOM, and Chemical Physics Letters and another has been submitted to the Journal of Applied Physics. The chapters are arranged as follows. Chapter 2 gives a more in-depth explanation of the structure and current state of research for the skutterudite and 2D TMD materials systems while chapter 3 explains the methodology and theory behind the models employed in this work. Chapter 4 includes the investigation of $\mathrm{Ca} / \mathrm{Ce}$ filler atoms in $\mathrm{FeSb}_{3}$ and $\mathrm{Te} / \mathrm{Ge}$ substitutional dopants on the $\mathrm{Sb}$ site in $\mathrm{CoSb}_{3} .{ }^{1}$ The work in chapter 5 screens a range of transition metals in 2D TMD materials comparing common oxidation states, atomic 
masses, and atomic radii. ${ }^{2}$ Chapter 6 investigates the effects of metal-site substitutional dopants in $2 \mathrm{D} \mathrm{MoS}_{2}$ and $\mathrm{WS}_{2}$ sheets. The work presented in this chapter is divided into two subsections: dopant concentration ${ }^{3}$ and dopant type. ${ }^{4}$ Chapter 7 investigates the effect of heterostructure formation between $2 \mathrm{D} \mathrm{MoS} 2$ and $\mathrm{MS}_{2}(\mathrm{M}=\mathrm{Cr}, \mathrm{Sc}, \mathrm{Ti}, \mathrm{W})$. Chapter 8 summarizes and concludes this dissertation. 


\section{References}

(1) Williamson, I.; Her, L. J. Y.; Su, X. L.; Yan, Y. G.; Wong-Ng, W.; Li, L. Improved thermoelectric performance of $(\mathrm{Fe}, \mathrm{Co}) \mathrm{Sb}$-3-type skutterudites from first-principles. J Appl Phys 2016, 119, 055101.

(2) Williamson, I.; Correa Hernandez, A.; Wong-Ng, W.; Li, L. HighThroughput Computational Screening of Electrical and Phonon Properties of Two-Dimensional Transition Metal Dichalcogenides. JOM 2016, 68, 2666.

(3) Williamson, I.; Li, S.; Correa Hernandez, A.; Lawson, M.; Chen, Y.; Li, L. Structural, electrical, phonon, and optical properties of Ti- and V-doped twodimensional MoS2. Chemical Physics Letters 2017, 674, 157.

(4) Williamson, I.; Wilson, N.; Lawson, M.; Li, S.; Chen, Y.; Li, L. Computational Screening of Metal-Site Dopants in Two-Dimensional MX2 (M = Mo, W; X = S, Se, Te). J Appl Phys 2017, submitted. 


\section{CHAPTER TWO: MATERIALS SYSTEMS}

The materials of interest in this dissertation are bulk skutterudites and twodimensional transition metal dichalcogenides (2D TMDs). Both offer a variety of compositional options and tailorable structures and geometries which can significantly affect materials properties and performance, specifically in electronic and energy applications. High-throughput computational screening methods were employed to analyze the effects of doping (including filler atoms and substitutions) and heterostructures for the two materials that are of interest.

\subsection{Skutterudites}

Skutterudite compounds are low-cost and easy to process materials with intrinsically good electrical transport properties and tunable thermal transport properties. ${ }^{1-10}$ The term "skutterudite" refers to minerals that have a cobalt arsenide, $\mathrm{CoAs}_{3}$-type crystal structure. They form in space group $\operatorname{Im} 3$ and consist of cage-like structures with the general formula $\mathrm{AB}_{3}$, where $\mathrm{A}$ is a transition metal and $\mathrm{B}$ is a pnictogen atom. Figure 2.1 shows the crystal structure of a filled skutterudite featuring M filler atoms and $\mathrm{B}$ rings in an $\mathrm{A}$ sublattice, where $\mathrm{M}=\mathrm{La}, \mathrm{Te}, \mathrm{Ge}, \mathrm{Ba}$, $\mathrm{In}$, or $\mathrm{Yb} ; \mathrm{A}=\mathrm{Fe}$, $\mathrm{Ru}, \mathrm{Co}, \mathrm{Ni}$, or Os; and B = Sb, P, or As. Sb-based skutterudites are extensively studied due to their high mobility, atomic masses, electrical conductivity, and Seebeck coefficients. ${ }^{11}$ 


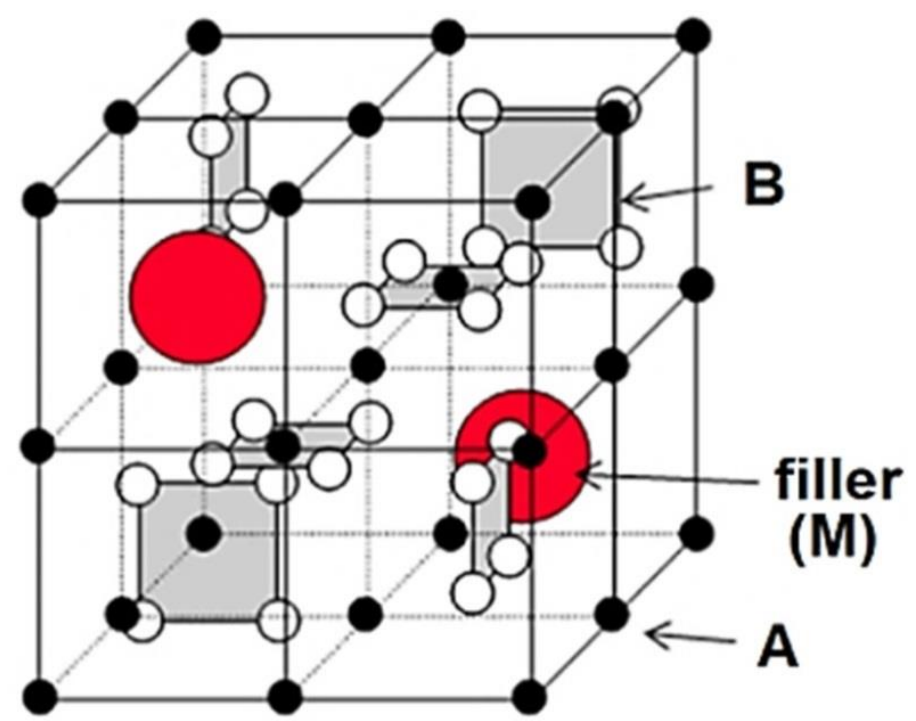

Figure 2.1. Crystal structure of a filled $M_{2} A_{8} B_{24}$ skutterudite with filler atom $M$ in red, $B$ in white, and $A$ in black. The gray substructures outlined by $B$ atoms are referred to as pnictogen rings.

Specifically, these novel characteristics make skutterudites of great interest for thermoelectric applications. Thermoelectric devices can be used to recover waste heat by converting it back into electrical power. This is known as the Seebeck effect. The performance metric of thermoelectric materials is known as the thermoelectric figure of merit,

$$
Z T=\frac{S^{2} \sigma}{\kappa} T
$$

where $S$ is the Seebeck coefficient, $\sigma$ is the electrical conductivity, the combined $S^{2} \sigma$ term is referred to as the power factor, $\kappa$ is the thermal conductivity, and $T$ is the absolute temperature. The implementation of thermoelectric devices has many promising commercial applications including in-home power and heating, automobile energy recovery, solar cells, and semiconductors. Unfortunately, it is difficult to obtain thermoelectric figures of merit that exceed unity (i.e., $Z T>1$ ) in macroscale devices. In order to improve these devices and bring them into large-scale use, there are a number of issues left to resolve. A greater understanding of transport phenomena is needed to 
overcome these obstacles. Skutterudite compounds generally have a ZT value around 1 and maintain large carrier concentrations and moderate Seebeck coefficients. ${ }^{12,13} \mathrm{CoSb}_{3}$ is of particular interest because of its high power factor and moderately high temperature stability. ${ }^{12}$ The most important task for improvement then comes from the reduction of thermal conductivity (without affecting carrier mobility).

\section{Current State of Skutterudite Materials Research}

The structure of a skutterudite has large degrees of freedom for doping and alloying, making them attractive for customization and design studies. A promising approach to improving skutterudite performance is therefore to modify their crystal structure by either filling structural voids with guest atoms or through substitutional dopants. ${ }^{11,14}$ The introduction of filler atoms has been widely studied because they offer increased electrical conductivity with negligible effects on Seebeck coefficient and decreased thermal phonon conductivity through a "rattling effect." 15 Calculations performed by Feldman et al., ${ }^{16}$ and eventually experimental work by Koza et al., ${ }^{17}$ have since challenged the rattling effect. Nevertheless, filler atoms can modify the phonon dispersion and ab initio calculations have shown a reduction in thermal conductivity through an increase in anharmonic scattering rates and reduction of group velocities. ${ }^{18}$ Alkaline earths have previously been used as fillers ${ }^{2}$ and Yang et al. ${ }^{19}$ have shown that a double-filled skutterudite, having one alkaline earth and one lanthanide (Ba and $\mathrm{Ce}$ ) filler, is even more effective at reducing the lattice thermal conductivity than using two alkaline earths (Ba and Sr). Previous experimental work from our collaborators ${ }^{20}$ used $^{\mathrm{Ca}}$ and $\mathrm{Ce}$ fillers (which have a greater mass difference, $71 \%$, than that of $\mathrm{Ba}$ and $\mathrm{Ce}, 2 \%$ ) and found that for the double-filled $\left(\mathrm{Ca}_{x} \mathrm{Ce}_{1-x}\right) \mathrm{Fe}_{4} \mathrm{Sb}_{12}$ series $(x=0,0.25,0.5,0.75$, and 
1 ), only the $x=0.5$ compound was stable. They also measured this compound to have a higher $Z T$ value than that of the end members due to its decreased thermal conductivity.

Alternatively, substitutional doping has been shown to improve skutterudite performance. Co site substitutions that have been investigated for the $\mathrm{CoSb}_{3}$ structure include $\mathrm{Cr},{ }^{21} \mathrm{Ni},{ }^{22}$ or $\mathrm{Fe}^{20}$ These substitutions reveal significant changes in transport properties such as an increase in electrical conductivity and a decrease in thermal conductivity; however, experimental and theoretical calculations have shown that substitution on the pnictogen rings ( $\mathrm{Sb}$ site) is more effective for reducing thermal conductivity due to the rings' dominance of the phonon thermal conductivity spectrum. ${ }^{16,23,24}$ Laser flash thermal diffusivity measurements on $\mathrm{CoSb}_{3-\mathrm{x}} \mathrm{Te}_{\mathrm{x}}$ compounds have shown a reduction in lattice thermal conductivity through atomic mass fluctuation and size strain. ${ }^{25}$ This effect is increased through the incorporation of electric-charge compensating Ge and Sn substitution on the Sb site which disrupt the bonding and symmetry of the rings. ${ }^{26}$

Despite the amount of research conducted for this material system, there have not been major improvements in the thermoelectric figure of merit for skutterudites since the early 1990s. Therefore, a fundamental investigation of fillers and substitutions and their effects on the electrical and phonon properties of skutterudite compounds is important for materials development.

\subsection{Two-Dimensional Transition Metal Dichalcogenides (2D TMDs)}

The variability and interesting transport properties inherent in skutterudites makes them a great benchmark material for building a transport properties modeling scheme. 
The approach can be translated to study similar phenomena in other materials. Here, we turned to a lower dimensionality and investigated 2D TMDs.

TMDs, having the chemical formula $\mathrm{MX}_{2}(\mathrm{M}=$ transition metal, $\mathrm{X}=$ chalcogen, e.g., $\mathrm{S}, \mathrm{Se}, \mathrm{Te}$ ), have received attention over the years for having interesting magnetic ${ }^{27,28}$ and photoelectric ${ }^{29}$ properties. Bulk three-dimensional transition metal dichalcogenides (3D TMDs) can exist as layered structures wherein each layer consists of a transition metal (M) sheet sandwiched between two chalcogen (X) sheets. These layers exist in one of two structure types: $2 \mathrm{H}$-type having $D_{6 h}$ point group symmetry and 1T-type having $D_{3 d}$ point group symmetry (see Figure 2.2). Strong covalent bonding exists within the $\mathrm{MX}_{2}$ layers (along the $a$ and $b$ axes) while much weaker van der Waals (vdW) interactions exist between the layers (along the $c$ axis). This allows individual $\mathrm{MX}_{2}$ layers to be separated via mechanical exfoliation. ${ }^{30}$
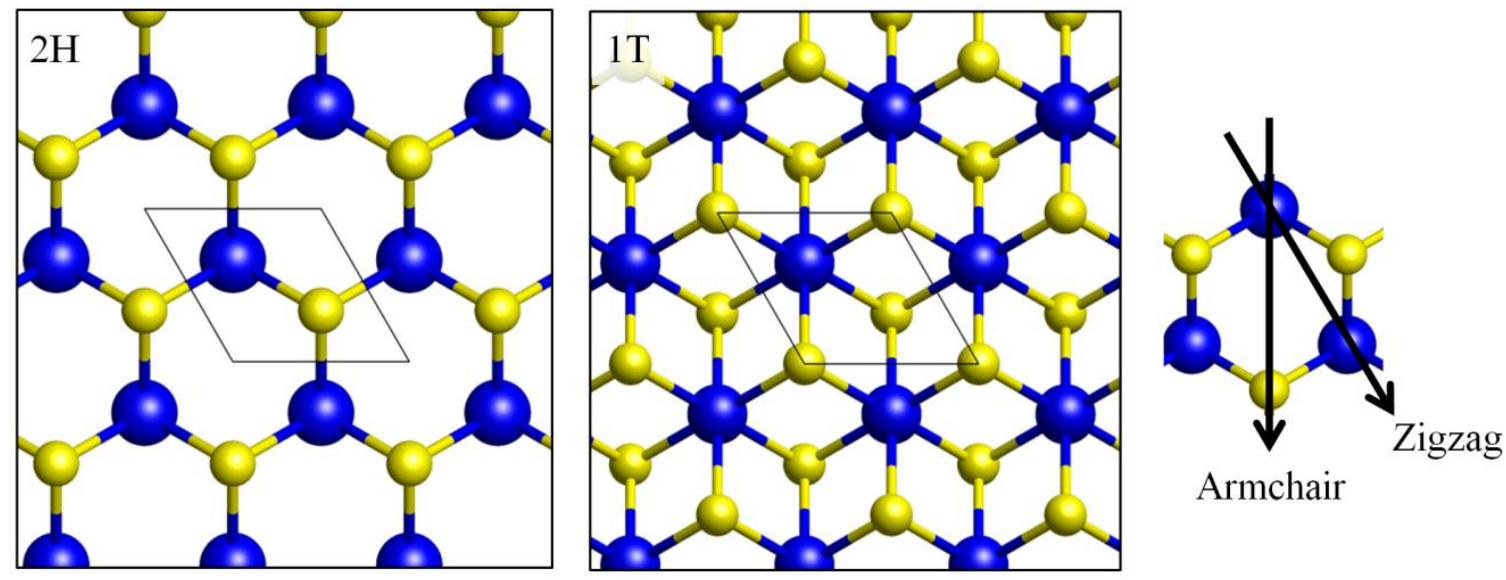

Figure 2.2. Top-down view of 2D TMD sheets having 2H- or 1T-type structures. Cyan and yellow spheres represent $M$ and $X$ atoms, respectively. Thin black lines frame the unit cell. Armchair and zigzag directions are depicted with bold black arrows. 
The separated 2D TMD materials have recently attracted much interest for a variety of applications including electronics, ${ }^{31-35}$ optoelectronics, ${ }^{36-39}$ catalysts, ${ }^{40,41}$ and thermoelectrics. ${ }^{42-45}$ In addition, the quantum confinement effect and sharp features in the electronic density of states (DoS) are beneficial for improving thermoelectric properties, such as increasing the Seebeck coefficient, $S$, in 2D TMDs compared to that of the bulk structures. ${ }^{46-48}$ It is also predicted that the lower dimensionality increases diffusive boundary scattering, effectively reducing the thermal conductivity, $\kappa$. Lowering the dimensionality of $\mathrm{Si}$, for example, has shown strong reductions in $\kappa$ and implications for enhanced thermoelectric performance. ${ }^{49,50}$ With a large number of potential 2D TMD compounds, it is important to identify key structure-property-performance relationships in order to screen preferred materials.

2D TMD materials are of particular interest for applications in electronic devices. With their wide compositional variety comes a variety of electronic structures including metals and direct or indirect bandgap semiconductors. The lower dimensionality further expands the range of properties beyond that of the bulk TMD materials. For example, bulk $\mathrm{MoS}_{2}$ has an indirect bandgap of $1.2 \mathrm{eV}$ while $2 \mathrm{D} \mathrm{MoS}_{2}$ has a direct bandgap of 1.8 eV. ${ }^{51,52}$ Reduced dimensionality also offers the potential for smaller device features and has been shown to greatly reduce short-channel effects, increase the on-off ratio, and reduce switching voltage in field-effect transistors (FETs) ${ }^{33,36}$ With these characteristics, 2D TMDs could potentially substitute for $\mathrm{Si}$ in electronic devices. ${ }^{53-55}$ Each of these factors contributes to the strong motivation for further understanding the behavior of $2 \mathrm{D}$ TMDs. 


\section{Current State of TMD Materials Research}

TMD studies focus on the stability and electronic structure of 2D TMDs and TMOs (transition metal dioxides) using density functional theory (DFT). ${ }^{31,32}$ These offer a general analysis of trends across a broad range of compositions and identify most structures to be semiconducting with small indirect bandgaps. Rasmussen et al. ${ }^{32}$ found that only $\mathrm{CrX}_{2}, \mathrm{MoX}_{2}$, and $\mathrm{WX}_{2}$ systems exhibit direct bandgaps. From these works, it is useful to identify more favorable 2D TMD compositions in terms of stability, electrical properties, and optical properties; however, none have been directed at what compositions are possible. Graedel et al. ${ }^{56}$ developed a method for quantifying the environmental implications, supply risk, and vulnerability to supply restrictions for 62 transition metals and metalloids. These "criticality scores" can be used to identify preferred materials and narrow the focus for screening potential compositions in order to develop high-performance, cost-effective, and environmentally friendly materials. Along with reduced dimensionality and identification of novel compositions and structures, strategies for further improvement of 2D TMD properties and performance include doping and heterostructure formation..$^{57,58}$

Metal-site substitutional dopants yield interesting changes in the intrinsic properties of 2D TMDs. These dopants can cause enhancements such as inducing magnetism ${ }^{59-62}$ and tuning of the electrical conductivity. ${ }^{62}$ They also offer the ability to modify the bandgap and photoluminescence ${ }^{63}$ and control whether it is a $p$ - or $n$-type semiconductor. ${ }^{64}$ Experimental work has shown that these dopants are stable in 2D TMDs and that they are a substitutional dopant rather than an interstitial impurity. ${ }^{65-68}$ 
This type of defect tends to be more stable because the dopant inherits the strong covalent bonds within the TMD sheet.

The formation of lateral heterostructures is another promising approach for improving optical and electrical properties. ${ }^{57}$ Lateral epitaxy has been used to synthesize high quality heterostructures with type II (staggered) band alignment which has potential for optoelectronic applications. ${ }^{69,70}$ Raman spectroscopy techniques have been used to analyze both the physical and phonon structures of heterostructures. ${ }^{71}$ Experimental work reveals that 2D TMDs prefer to form heterostructures along the "zigzag" direction of the honeycomb structure (see Figure 2.2). ${ }^{72}$ First-principles calculations have found interesting electronic structure effects of these zigzag junctions. For example, the bandgap of the lateral $\mathrm{MoS}_{2}-\mathrm{WS}_{2}$ heterostructure has been calculated to be about 1.58 $\mathrm{eV}$, smaller than that of $\mathrm{MoS}_{2}$ and $\mathrm{WS}_{2} \cdot{ }^{73}$ Band offset calculations confirm type II band alignment between $\mathrm{MoX}_{2}-\mathrm{WX}_{2}$ heterostructures and reveal an increase in both the conduction band minimum $(\mathrm{CBM})$ and valence band maximum (VBM) with increasing $\mathrm{M}$ and $\mathrm{X}$ atomic numbers. ${ }^{74}$

A thorough investigation into the structural, electrical, and phonon properties of 2D TMDs is crucial for the development of these materials. This can be achieved by identifying novel compositions and understanding the effects of substitutional dopants and heterostructures.

\subsection{Computational Modeling Approaches}

The choice of computational modeling technique can be based on a number of factors including: length scale, what variables are needed/relevant (e.g., time, temperature), and the governing physics of the properties in question. For example, an 
investigation of bonding characteristics requires the use of quantum mechanics and a very small length scale (nanometer) whereas thermal energy propagation in a nuclear reactor core may be on the order of meters and involve a continuum modeling approach. For this dissertation, the scope is narrowed to smaller length scales and methods that can be used to investigate electrical and phonon properties.

Equilibrium molecular dynamics (EMD) is one approach that can be used to calculate transport coefficients via the Green-Kubo formula (GKF). ${ }^{75,76}$ This is a widely studied approach due to its ability to yield accurate thermal properties of large materials; ${ }^{77-79}$ however, it does not account for electrical properties and is not well suited for $2 \mathrm{D}$ materials. For investigating the nanoscale regime, the use of density functional theory (DFT) is preferred because it incorporates electron density.

In this dissertation, the quantum mechanics-based method of DFT was employed to investigate these materials. Within DFT, there are a number of options for calculating transport properties of a material. Traditionally, DFT has been shown to underestimate bandgaps ${ }^{80}$ so many approaches have been proposed for improving band structure calculations. Among the more rigorous calculations include the use of hybrid functionals ${ }^{81-84}$ which incorporate some exact exchange into the exchange correlation interaction term and the $G W$ approximation ${ }^{85}$ which involves the expansion of selfenergies with respect to the single-particle Green's function, $G$, and the screened Coulomb interaction, $W$. These calculations have the advantage of generating more accurate band structures without relying on empirical data; however, they greatly increase computational costs and are not easily suited for screening purposes. The semi-empirical method of DFT $+\mathrm{U},{ }^{86}$ which adds a Hubbard-like on-site Coulomb potential term to 
account for strongly correlated electronic states, can be quickly and easily implemented to correct the overestimation and produce good quality band structures with negligible computational costs.

The calculation of thermal conductivity from first-principles is made possible through the full linearization of the Boltzmann transport equation for phonons. ${ }^{87}$ This is a very rigorous approach and involves the calculation of third-order force constants to account for phonon anharmonicity within the material. The computational costs are too high for screening purposes and it is not yet fully implemented for 2D materials; however, second-order force constant calculations can account for normal processes with only moderate computational costs. The use of density functional perturbation theory $(\mathrm{DFPT})^{88}$ allows for the calculation of necessary force constants and can produce phonon dispersion and density of states (DoS). This does not allow for the calculation of thermal conductivity but can provide useful phonon property information for materials screening. This dissertation employs the use of DFT to screen structural, bonding, electrical, and phonon properties of 3D skutterudite and 2D TMD materials focusing on the effects of doping and heterostructures. The problem of understanding electrical and thermal transport in these materials is one of multiscale and multiphysics. There remains a lack of general knowledge that must be addressed through the use of large-scale materials screening processes before more-rigorous calculations can be utilized. More information about the methods used in this work can be found in Chapter 3. 


\section{References}

(1) Tan, G. J.; Wang, S. Y.; Li, H.; Yan, Y. G.; Tang, X. F. Enhanced thermoelectric performance in zinc substituted p-type filled skutterudites CeFe4xZnxSb12. Journal of Solid State Chemistry 2012, 187, 316.

(2) Dyck, J. S.; Chen, W. D.; Uher, C.; Chen, L.; Tang, X. F.; Hirai, T. Thermoelectric properties of the n-type filled skutterudite Ba0.3Co4Sb12 doped with Ni. J Appl Phys 2002, 91, 3698.

(3) Wong-Ng, W.; Kaduk, J. A.; Tan, G.; Yan, Y.; Tang, X. X-ray powder reference patterns of the $\mathrm{Fe}(\mathrm{Sb} 2+x \mathrm{Te} 1-\mathrm{x})$ skutterudites for thermoelectric applications. Powder Diffraction 2014, 29, 260.

(4) Tan, G. J.; Liu, W.; Chi, H.; Su, X. L.; Wang, S. Y.; Yan, Y. G.; Tang, X. F.; Wong-Ng, W.; Uher, C. Realization of high thermoelectric performance in ptype unfilled ternary skutterudites FeSb2+xTe1-x via band structure modification and significant point defect scattering. Acta Materialia 2013, 61, 7693.

(5) Yan, Y. G.; Wong-Ng, W.; Kaduk, J. A.; Tan, G. J.; Xie, W. J.; Tang, X.

F. Correlation of thermoelectric and microstructural properties of $\mathrm{p}$-type CeFe4Sb12 melt-spun ribbons using a rapid screening method. Appl Phys Lett 2011, 98 .

(6) He, T.; Chen, J. Z.; Rosenfeld, H. D.; Subramanian, M. A. Thermoelectric properties of indium-filled skutterudites. Chem Mater 2006, 18, 759.

(7) Huang, B.; Kaviany, M. Filler-reduced phonon conductivity of thermoelectric skutterudites: Ab initio calculations and molecular dynamics simulations. Acta Materialia 2010, 58, 4516.

(8) Hara, R.; Inoue, S.; Kaibe, H. T.; Sano, S. Aging effects of large-size ntype CoSb3 prepared by spark plasma sintering. Journal of Alloys and Compounds 2003, 349, 297. 
(9) Kim, H.; Kaviany, M.; Thomas, J. C.; Van der Ven, A.; Uher, C.; Huang, B. L. Structural Order-Disorder Transitions and Phonon Conductivity of Partially Filled Skutterudites. Phys Rev Lett 2010, 105, 265901.

(10) Luo, P. F.; Tang, X. F.; Xiong, C.; Zhang, Q. J. Effect of multiwalled carbon nanotubes on the thermoelectric properties of p-type $\mathrm{Ba} 0.3 \mathrm{FeCo} 3 \mathrm{Sb} 12$ compounds. Acta Phys Sin-Ch Ed 2005, 54, 2403.

(11) Rull-Bravo, M.; Moure, A.; Fernandez, J. F.; Martin-Gonzalez, M. Skutterudites as thermoelectric materials: revisited. Rsc Adv 2015, 5, 41653.

(12) Morelli, D. T.; Caillat, T.; Fleurial, J. P.; Borshchevsky, A.; Vandersande, J.; Chen, B.; Uher, C. Low-Temperature Transport-Properties of P-Type Cosb3. Phys Rev B 1995, 51, 9622.

(13) Caillat, T.; Borshchevsky, A.; Fleurial, J. P. Properties of single crystalline semiconducting CoSb3. J Appl Phys 1996, 80, 4442.

(14) Sootsman, J. R.; Chung, D. Y.; Kanatzidis, M. G. New and Old Concepts in Thermoelectric Materials. Angew Chem Int Edit 2009, 48, 8616.

(15) Slack, G. A.; Tsoukala, V. G. Some Properties of Semiconducting Irsb3. J Appl Phys 1994, 76, 1665.

(16) Feldman, J. L.; Singh, D. J. Lattice dynamics of skutterudites: Firstprinciples and model calculations for CoSb3. Phys Rev B 1996, 53, 6273.

(17) Koza, M. M.; Johnson, M. R.; Viennois, R.; Mutka, H.; Girard, L.; Ravot, D. Breakdown of phonon glass paradigm in La- and Ce-filled $\mathrm{Fe}(4) \mathrm{Sb}(12)$ skutterudites. Nature Materials 2008, 7, 805.

(18) Li, W.; Mingo, N. Thermal conductivity of fully filled skutterudites: Role of the filler. Phys Rev B 2014, 89, 184304.

(19) Yang, J.; Zhang, W.; Bai, S. Q.; Mei, Z.; Chen, L. D. Dual-frequency resonant phonon scattering in BaxRyCo4Sb12 (R=La, Ce, and Sr). Appl Phys Lett 2007, 90, 192111. 
(20) Yan, Y. G.; Wong-Ng, W.; Li, L.; Levin, I.; Kaduk, J. A.; Suchomel, M. R.; Sun, X.; Tan, G. J.; Tang, X. F. Structures and thermoelectric properties of double-filled (CaxCe1-x)Fe4Sb12 skutterudites. Journal of Solid State Chemistry 2014, 218.

(21) Yang, J.; Endres, M. G.; Meisner, G. P. Valence of Cr in skutterudites: Electrical transport and magnetic properties of Cr-doped CoSb3. Phys Rev B 2002, 66 .

(22) Yang, J.; Morelli, D. T.; Meisner, G. P.; Chen, W.; Dyck, J. S.; Uher, C. Influence of electron-phonon interaction on the lattice thermal conductivity of Co1-xNixSb3. Phys Rev B 2002, 65.

(23) Dimitrov, I. K.; Manley, M. E.; Shapiro, S. M.; Yang, J.; Zhang, W.; Chen, L. D.; Jie, Q.; Ehlers, G.; Podlesnyak, A.; Camacho, J.; Li, Q. A. Einstein modes in the phonon density of states of the single-filled skutterudite Yb0.2Co4Sb12. Phys Rev B 2010, 82, 174301.

(24) Chi, H.; Kim, H.; Thomas, J. C.; Su, X. L.; Stackhouse, S.; Kaviany, M.; Van der Ven, A.; Tang, X. F.; Uher, C. Configuring pnicogen rings in skutterudites for low phonon conductivity. Phys Rev B 2012, 86, 195209.

(25) Liu, W. S.; Zhang, B. P.; Zhao, L. D.; Li, J. F. Improvement of Thermoelectric Performance of CoSb3-xTex Skutterudite Compounds by Additional Substitution of IVB-Group Elements for Sb. Chem Mater 2008, 20, 7526.

(26) Su, X. L.; Li, H.; Wang, G. Y.; Chi, H.; Zhou, X. Y.; Tang, X. F.; Zhang, Q. J.; Uher, C. Structure and Transport Properties of Double-Doped CoSb2.75Ge0.25-xTex (x=0.125-0.20) with in Situ Nanostructure. Chem Mater 2011, 23, 2948.

Ogawa, S. Magnetic-Properties of 3d Transition-Metal Dichalcogenides with the Pyrite Structure. J Appl Phys 1979, 50, 2308.

(28) Shishidou, T.; Freeman, A. J.; Asahi, R. Effect of GGA on the halfmetallicity of the itinerant ferromagnet CoS2. Phys Rev B 2001, 64, 180401. 
(29) Tributsch, H. Solar Energy-Assisted Electrochemical Splitting of Water. Z Naturforsch A 1977, 32, 972.

(30) Coleman, J. N.; Lotya, M.; O'Neill, A.; Bergin, S. D.; King, P. J.; Khan, U.; Young, K.; Gaucher, A.; De, S.; Smith, R. J.; Shvets, I. V.; Arora, S. K.; Stanton, G.; Kim, H. Y.; Lee, K.; Kim, G. T.; Duesberg, G. S.; Hallam, T.; Boland, J. J.; Wang, J. J.; Donegan, J. F.; Grunlan, J. C.; Moriarty, G.; Shmeliov, A.; Nicholls, R. J.; Perkins, J. M.; Grieveson, E. M.; Theuwissen, K.; McComb, D. W.; Nellist, P. D.; Nicolosi, V. Two-Dimensional Nanosheets Produced by Liquid Exfoliation of Layered Materials. Science 2011, 331, 568.

(31) Ataca, C.; Sahin, H.; Ciraci, S. Stable, Single-Layer MX2 TransitionMetal Oxides and Dichalcogenides in a Honeycomb-Like Structure. Journal of Physical Chemistry C 2012, 116, 8983.

(32) Rasmussen, F. A.; Thygesen, K. S. Computational 2D Materials Database: Electronic Structure of Transition-Metal Dichalcogenides and Oxides. Journal of Physical Chemistry C 2015, 119, 13169.

(33) Butler, S. Z.; Hollen, S. M.; Cao, L. Y.; Cui, Y.; Gupta, J. A.; Gutierrez, H. R.; Heinz, T. F.; Hong, S. S.; Huang, J. X.; Ismach, A. F.; Johnston-Halperin, E.; Kuno, M.; Plashnitsa, V. V.; Robinson, R. D.; Ruoff, R. S.; Salahuddin, S.; Shan, J.; Shi, L.; Spencer, M. G.; Terrones, M.; Windl, W.; Goldberger, J. E. Progress, Challenges, and Opportunities in Two-Dimensional Materials Beyond Graphene. Acs Nano 2013, 7, 2898.

(34) McDonnell, S.; Addou, R.; Buie, C.; Wallace, R. M.; Hinkle, C. L. DefectDominated Doping and Contact Resistance in MoS2. Acs Nano 2014, 8, 2880.

(35) Gong, C.; Colombo, L.; Wallace, R. M.; Cho, K. The Unusual Mechanism of Partial Fermi Level Pinning at Metal-MoS2 Interfaces. Nano Lett 2014, 14, 1714.

(36) Ganatra, R.; Zhang, Q. Few-Layer MoS2: A Promising Layered Semiconductor. Acs Nano 2014, 8, 4074. 
(37) Radisavljevic, B.; Radenovic, A.; Brivio, J.; Giacometti, V.; Kis, A. Single-layer MoS2 transistors. Nat Nanotechnol 2011, 6, 147.

(38) Lopez-Sanchez, O.; Lembke, D.; Kayci, M.; Radenovic, A.; Kis, A. Ultrasensitive photodetectors based on monolayer MoS2. Nat Nanotechnol 2013, $8,497$.

(39) Baugher, B. W. H.; Churchill, H. O. H.; Yang, Y. F.; Jarillo-Herrero, P. Optoelectronic devices based on electrically tunable $\mathrm{p}$-n diodes in a monolayer dichalcogenide. Nat Nanotechnol 2014, 9, 262.

(40) Chen, W.; Santos, E. J. G.; Zhu, W. G.; Kaxiras, E.; Zhang, Z. Y. Tuning the Electronic and Chemical Properties of Monolayer MoS2 Adsorbed on Transition Metal Substrates. Nano Lett 2013, 13, 509.

(41) Jaramillo, T. F.; Jorgensen, K. P.; Bonde, J.; Nielsen, J. H.; Horch, S.; Chorkendorff, I. Identification of active edge sites for electrochemical H-2 evolution from MoS2 nanocatalysts. Science 2007, 317, 100.

(42) Huang, W.; Da, H. X.; Liang, G. C. Thermoelectric performance of MX2 (M = Mo, W; X = S, Se) monolayers. J Appl Phys 2013, 113, 104304.

(43) Huang, W.; Luo, X.; Gan, C. K.; Quek, S. Y.; Liang, G. C. Theoretical study of thermoelectric properties of few-layer MoS2 and WSe2. Physical Chemistry Chemical Physics 2014, 16, 10866.

(44) Lee, C.; Hong, J.; Whangbo, M. H.; Shim, J. H. Enhancing the Thermoelectric Properties of Layered Transition-Metal Dichalcogenides 2HMQ(2) (M = Mo, W; Q = S, Se, Te) by Layer Mixing: Density Functional Investigation. Chem Mater 2013, 25, 3745.

(45) Gu, X. K.; Yang, R. G. Phonon transport in single-layer transition metal dichalcogenides: A first-principles study. Appl Phys Lett 2014, 105, 131903.

(46) Hicks, L. D.; Dresselhaus, M. S. Effect of Quantum-Well Structures on the Thermoelectric Figure of Merit. Phys Rev B 1993, 47, 12727. 
(47) Hicks, L. D.; Dresselhaus, M. S. Thermoelectric Figure of Merit of a OneDimensional Conductor. Phys Rev B 1993, 47, 16631.

(48) Mahan, G. D.; Sofo, J. O. The best thermoelectric. P Natl Acad Sci USA 1996, 93, 7436 .

(49) Hochbaum, A. I.; Chen, R. K.; Delgado, R. D.; Liang, W. J.; Garnett, E. C.; Najarian, M.; Majumdar, A.; Yang, P. D. Enhanced thermoelectric performance of rough silicon nanowires. Nature 2008, 451, 163.

(50) Boukai, A. I.; Bunimovich, Y.; Tahir-Kheli, J.; Yu, J. K.; Goddard, W. A.; Heath, J. R. Silicon nanowires as efficient thermoelectric materials. Nature 2008, $451,168$.

(51) Kam, K. K.; Parkinson, B. A. Detailed Photocurrent Spectroscopy of the Semiconducting Group-Vi Transition-Metal Dichalcogenides. J Phys Chem-Us 1982, 86, 463.

(52) Mak, K. F.; Lee, C.; Hone, J.; Shan, J.; Heinz, T. F. Atomically Thin MoS2: A New Direct-Gap Semiconductor. Phys Rev Lett 2010, 105, 136805.

(53) Roy, K.; Padmanabhan, M.; Goswami, S.; Sai, T. P.; Ramalingam, G.; Raghavan, S.; Ghosh, A. Graphene-MoS2 hybrid structures for multifunctional photoresponsive memory devices. Nat Nanotechnol 2013, 8, 826.

(54) Schmidt, H.; Wang, S. F.; Chu, L. Q.; Toh, M.; Kumar, R.; Zhao, W. J.; Neto, A. H. C.; Martin, J.; Adam, S.; Ozyilmaz, B.; Eda, G. Transport Properties of Monolayer MoS2 Grown by Chemical Vapor Deposition. Nano Lett 2014, 14, 1909.

(55) Yu, L. L.; Lee, Y. H.; Ling, X.; Santos, E. J. G.; Shin, Y. C.; Lin, Y. X.; Dubey, M.; Kaxiras, E.; Kong, J.; Wang, H.; Palacios, T. Graphene/MoS2 Hybrid Technology for Large-Scale Two-Dimensional Electronics. Nano Lett 2014, 14, 3055 .

(56) Graedel, T. E.; Harper, E. M.; Nassar, N. T.; Nuss, P.; Reck, B. K. Criticality of metals and metalloids. P Natl Acad Sci USA 2015, 112, 4257. 
Wang, H. T.; Yuan, H. T.; Hong, S. S.; Li, Y. B.; Cui, Y. Physical and chemical tuning of two-dimensional transition metal dichalcogenides. Chem Soc Rev 2015, 44, 2664.

Liu, G. B.; Xiao, D.; Yao, Y. G.; Xu, X. D.; Yao, W. Electronic structures and theoretical modelling of two-dimensional group-VIB transition metal dichalcogenides. Chem Soc Rev 2015, 44, 2643.

(59) Zhou, Y. G.; Su, Q. L.; Wang, Z. G.; Deng, H. Q.; Zu, X. T. Controlling magnetism of MoS2 sheets by embedding transition-metal atoms and applying strain. Physical Chemistry Chemical Physics 2013, 15, 18464.

(60) Cheng, Y. C.; Zhu, Z. Y.; Mi, W. B.; Guo, Z. B.; Schwingenschlogl, U. Prediction of two-dimensional diluted magnetic semiconductors: Doped monolayer MoS2 systems. Phys Rev B 2013, 87, 100401.

(61) Ramasubramaniam, A.; Naveh, D. Mn-doped monolayer MoS2: An atomically thin dilute magnetic semiconductor. Phys Rev B 2013, 87, 195201.

(62) Saab, M.; Raybaud, P. Tuning the Magnetic Properties of MoS2 Single Nanolayers by 3d Metals Edge Doping. Journal of Physical Chemistry C 2016, 120, 10691.

(63) Chen, Y. F.; Xi, J. Y.; Dumcenco, D. O.; Liu, Z.; Suenaga, K.; Wang, D.; Shuai, Z. G.; Huang, Y. S.; Xie, L. M. Tunable Band Gap Photoluminescence from Atomically Thin Transition-Metal Dichalcogenide Alloys. Acs Nano 2013, 7,4610 .

(64) Suh, J.; Park, T. E.; Lin, D. Y.; Fu, D. Y.; Park, J.; Jung, H. J.; Chen, Y. B.; Ko, C.; Jang, C.; Sun, Y. H.; Sinclair, R.; Chang, J.; Tongay, S.; Wu, J. Q. Doping against the Native Propensity of MoS2: Degenerate Hole Doping by Cation Substitution. Nano Lett 2014, 14, 6976.

(65) Hsu, W. K.; Zhu, Y. Q.; Yao, N.; Firth, S.; Clark, R. J. H.; Kroto, H. W.; Walton, D. R. M. Titanium-doped molybdenum disulfide nanostructures. Advanced Functional Materials 2001, 11, 69. 
(66) Deepak, F. L.; Cohen, H.; Cohen, S.; Feldman, Y.; Popovitz-Biro, R.; Azulay, D.; Millo, O.; Tenne, R. Fullerene-like (IF) NbxMo1-xS2 nanoparticles. $J$ Am Chem Soc 2007, 129, 12549.

(67) Sun, Q. C.; Yadgarov, L.; Rosentsveig, R.; Seifert, G.; Tenne, R.; Musfeldt, J. L. Observation of a Burstein-Moss Shift in Rhenium-Doped MoS2 Nanoparticles. Acs Nano 2013, 7, 3506.

(68) Yadgarov, L.; Rosentsveig, R.; Leitus, G.; Albu-Yaron, A.; Moshkovich, A.; Perfilyev, V.; Vasic, R.; Frenkel, A. I.; Enyashin, A. N.; Seifert, G.; Rapoport, L.; Tenne, R. Controlled Doping of MS2 (M=W, Mo) Nanotubes and Fullerenelike Nanoparticles. Angew Chem Int Edit 2012, 51, 1148.

(69) Huang, C. M.; Wu, S. F.; Sanchez, A. M.; Peters, J. J. P.; Beanland, R.; Ross, J. S.; Rivera, P.; Yao, W.; Cobden, D. H.; Xu, X. D. Lateral heterojunctions within monolayer MoSe2-WSe2 semiconductors. Nature Materials 2014, 13, 1096.

(70) Chen, K.; Wan, X.; Wen, J. X.; Xie, W. G.; Kang, Z. W.; Zeng, X. L.; Chen, H. J.; Xu, J. B. Electronic Properties of MoS2-WS2 Heterostructures Synthesized with Two-Step Lateral Epitaxial Strategy. Acs Nano 2015, 9, 9868.

(71) Zhang, X.; Qiao, X. F.; Shi, W.; Wu, J. B.; Jiang, D. S.; Tan, P. H. Phonon and Raman scattering of two-dimensional transition metal dichalcogenides from monolayer, multilayer to bulk material. Chem Soc Rev 2015, 44, 2757.

(72) Zhang, X. Q.; Lin, C. H.; Tseng, Y. W.; Huang, K. H.; Lee, Y. H. Synthesis of Lateral Heterostructures of Semiconducting Atomic Layers. Nano Lett 2015, 15, 410.

(73) Wang, Q. W.; Wu, P.; Cao, G. Y.; Huang, M. First-principles study of the structural and electronic properties of MoS2-WS2 and MoS2-MoTe2 monolayer heterostructures. J Phys D Appl Phys 2013, 46, 505308.

(74) Kang, J.; Tongay, S.; Zhou, J.; Li, J. B.; Wu, J. Q. Band offsets and heterostructures of two-dimensional semiconductors. Appl Phys Lett 2013, 102, 012111. 
(75) Green, M. S. Markoff Random Processes and the Statistical Mechanics of Time-Dependent Phenomena .2. Irreversible Processes in Fluids. Journal of Chemical Physics 1954, 22, 398.

(76) Kubo, R. Statistical-Mechanical Theory of Irreversible Processes .1. General Theory and Simple Applications to Magnetic and Conduction Problems. J Phys Soc Jpn 1957, 12, 570.

(77) Che, J. W.; Cagin, T.; Deng, W. Q.; Goddard, W. A. Thermal conductivity of diamond and related materials from molecular dynamics simulations. Journal of Chemical Physics 2000, 113, 6888.

(78) Volz, S. G.; Chen, G. Molecular-dynamics simulation of thermal conductivity of silicon crystals. Phys Rev B 2000, 61, 2651.

(79) Yao, Z. H.; Wang, J. S.; Li, B. W.; Liu, G. R. Thermal conduction of carbon nanotubes using molecular dynamics. Phys Rev B 2005, 71, 085417.

(80) Perdew, J. P. Density Functional Theory and the Band-Gap Problem. Int J Quantum Chem 1985, 497.

(81) Becke, A. D. A New Mixing of Hartree-Fock and Local DensityFunctional Theories. Journal of Chemical Physics 1993, 98, 1372.

(82) Perdew, J. P.; Emzerhof, M.; Burke, K. Rationale for mixing exact exchange with density functional approximations. Journal of Chemical Physics 1996, 105, 9982.

(83) Adamo, C.; Barone, V. Toward reliable density functional methods without adjustable parameters: The PBE0 model. Journal of Chemical Physics 1999, $110,6158$.

(84) Heyd, J.; Scuseria, G. E.; Ernzerhof, M. Hybrid functionals based on a screened Coulomb potential. Journal of Chemical Physics 2003, 118, 8207.

(85) Aryasetiawan, F.; Gunnarsson, O. The GW method. Rep Prog Phys 1998, $61,237$. 
(86) Liechtenstein, A. I.; Anisimov, V. I.; Zaanen, J. Density-functional theory and strong interactions: Orbital ordering in Mott-Hubbard insulators. Phys Rev B 1995, 52, R5467.

(87) Li, W.; Carrete, J.; Katcho, N. A.; Mingo, N. ShengBTE: A solver of the Boltzmann transport equation for phonons. Computer Physics Communications 2014, 185, 1747.

(88) Parlinski, K.; Li, Z. Q.; Kawazoe, Y. First-principles determination of the soft mode in cubic ZrO2. Phys Rev Lett 1997, 78, 4063. 


\section{CHAPTER THREE: MODELING ELECTRICAL AND THERMAL PROPERTIES IN THE SOLID STATE}

The field of computational materials modeling is a fast growing area that offers a means of prediction and understanding beyond that of conventional research. By applying numerical models to emulate physical phenomena, we can analyze more wholly the relationships between structure, properties, and performance. Through the utilization of high-performance computing facilities, rigorous and complex calculations can be conducted on numerous materials systems faster than experiments. This work demonstrates how density functional theory (DFT), in conjunction with Boltzmann transport theory and density functional perturbation theory (DFPT), can be implemented to analyze structures, energetics, electrical, and thermal transport properties of skutterudite and two dimensional transition metal dichalcogenides (2D TMDs).

The flowchart in Figure 3.1 is an overview of different computational methods used in this dissertation. All structure, energetic, electronic, and force calculations performed in this work begin with DFT and the total energy minimization calculation. After optimizing the appropriate DFT parameters (see the Approximations subsection in 3.1 Density Functional Theory (DFT)), the energetic and structural ground state can be determined. Section 3.2 Electrical Transport Properties explains how the band energies can then be expanded to calculate semi-classic transport coefficients such as $S, \sigma$, and $\kappa_{\mathrm{e}}$. Next, section 3.3 Thermal Transport Properties explains how the second-order force constants calculated with DFPT can be used to determine phonon dispersion and DoS 
spectra. Finally, the incorporation of the recently developed atomistic Green's function (AGF) method, along with the second-order force constants, can be used to calculate transmission coefficients across a 2D TMD heterojunction as well as thermal boundary conductance.

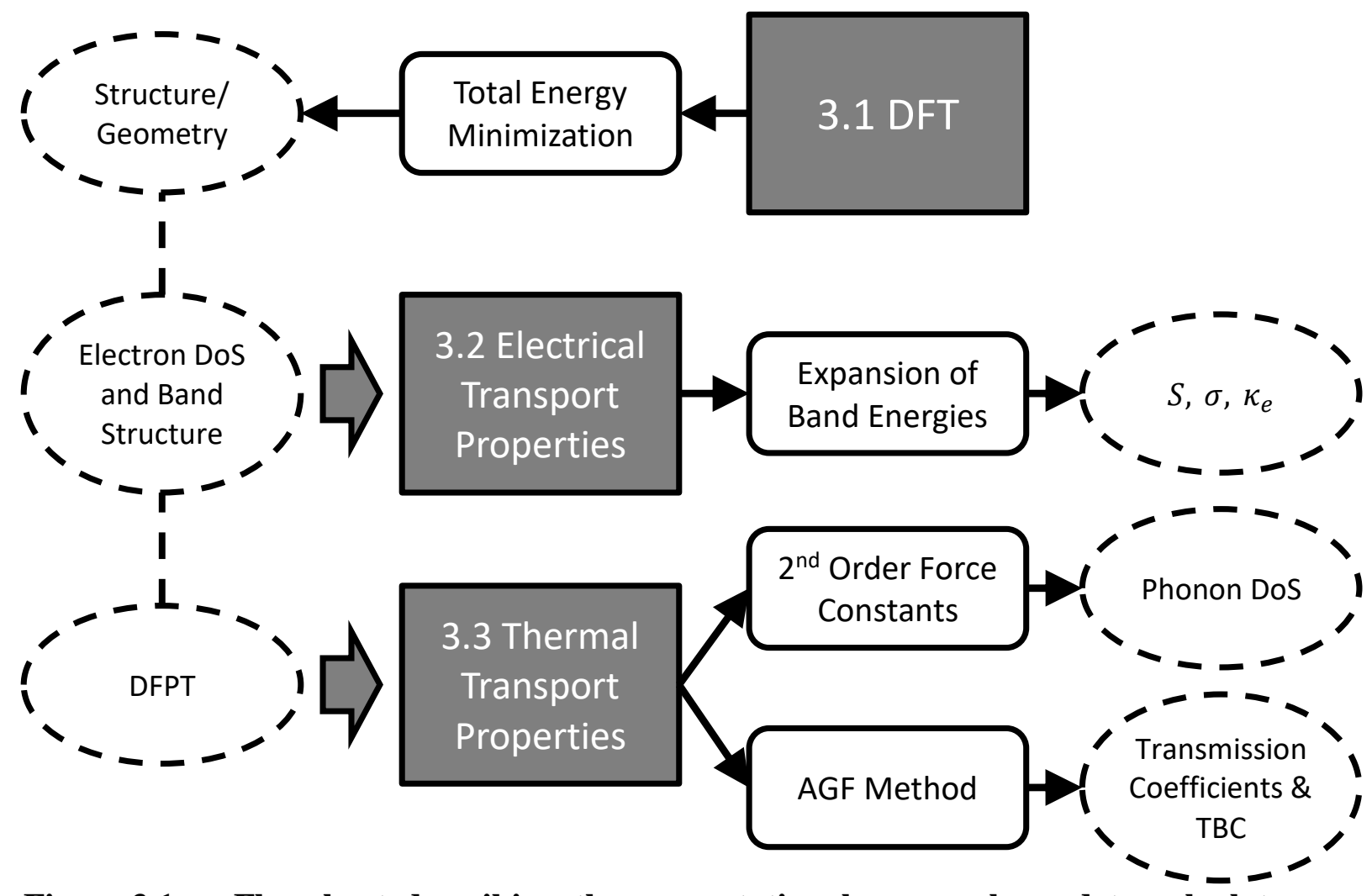

Figure 3.1. Flowchart describing the computational approach used to calculate electrical and thermal transport properties in this dissertation. DFT is used to minimize the total energy of the system in order to determine the electronic ground state. This can then be used to calculate structural features, band energies, and DFPT force constants. The band energies can be expanded to calculate electrical transport coefficients such as $S, \sigma$, and $\kappa_{\mathrm{e}}$. The second-order force constants can be used to calculate phonon DoS or combined with the AGF method to determine phonon transmission coefficients across a heterojunction.

\subsection{Density Functional Theory (DFT)}

The many-body problem describes the fact that wavefunctions needed to define a system having more than two electrons (up to infinity) range from extremely difficult to impossible to solve due to extensive particle interaction. DFT is an approach to handling 
the many-body problem by approximating the energy of a system by means of the charge density which is a function of the electronic wavefunctions. Despite a number of approximations, DFT can reasonably estimate the geometry and structure of various materials.

This approach revolves primarily around solving the Kohn-Sham (KS) equation, which is an adaptation of the Schrödinger equation for non-interacting particles.

$$
\widehat{H}_{K S} \Psi(\boldsymbol{r})=E(\boldsymbol{r}) \Psi(\boldsymbol{r})
$$

The symbol $\Psi$ is the electron wavefunction, $E$ is the total energy of the system, and $\widehat{H}_{K S}$ is the KS Hamiltonian operator defined by,

$$
\widehat{H}_{K S}=E_{k i n}^{n o n}+U_{e x t}+U_{H}+U_{x c}
$$

Here, the energy terms are broken down into $E_{\text {kin }}^{\text {non }}$,- the kinetic energy of the noninteracting electron $\left(-\frac{1}{2} \nabla^{2}\right), U_{\text {ext }}$ - the external potential felt by the electron from the nuclei, $U_{H}$ - the Hartree potential which assumes the electrons are independent and interact in an averaged way (alternatively known as the mean-field approximation), and $U_{x c}$ - the exchange correlation which approximates all interaction terms. This eigenvalue problem is solved self-consistently where a trial charge density is used to calculate a total energy for the system to compare with the starting value. The method is repeated until the energy is consistent within a given range. By calculating interatomic forces and iteratively translating atoms to minimize those forces, the ground state geometry can be determined. ${ }^{1}$

\section{Approximations}

There are four primary approximations considered in this dissertation that directly affect the calculations: supercell, exchange-correlation, pseudopotential theory, and the 
self-consistent field (scf) approach. To minimize error in the calculations and validate the models, each approximation was investigated via convergence tests. In general, this is done by running calculations with various parameters and comparing results (e.g., lattice constants, bond lengths, cell volume, and electronic bandgaps). Each approximation affects different aspects of the calculation so each is addressed differently.

The use of Bloch's theorem in DFT treats materials systems as periodic and repeating. The supercell approximation is used to handle aperiodic configurations such as dopants and surfaces. 2D systems in this dissertation are treated as single- or few-layer atomic sheets separated by large ( $>20 \AA$ ) vacuum layers. The choice of vacuum thickness came from convergence tests which showed negligible interaction between repeating sheets with this thickness.

Electron-electron interactions remain one of the most difficult aspects of DFT needed to accurately describe a system. Exchange-correlation is a term added to the $\widehat{H}_{K S}$ operator that accounts for all electron interaction terms. The simplest approach to calculating this is through the local density approximation (LDA) ${ }^{2}$ which assumes the exchange-correlation energy per electron at a given point is equal to the exchangecorrelation energy for a homogeneous electron gas of the same density. Alternately, the generalized gradient approximation (GGA) accounts for semi-local information by adding an additional term to account for the rate of change in exchange-correlation energy at a given point. This is known to produce slightly more accurate structures than LDA and is generally better suited for more materials systems. ${ }^{1}$ There are many different forms of GGA, but the method of Perdew, Burke, and Ernzerhof $(\mathrm{PBE})^{3}-$ which is commonly used for its simplicity, accuracy, and independence from empirical input - 
was chosen for this dissertation. Convergence tests confirmed that it is useful for determining skutterudite and 2D TMD structures.

The pseudopotential theory involves replacing the strong potential between electrons and ions with a much weaker pseudopotential, which are designed to maintain the features of the valence electrons while "freezing" and simplifying the core electrons. A graphical representation of the pseudopotential and pseudo wave function can be seen in Figure 3.2. A commonly used approach for generating pseudopotentials that is both efficient and accurate is called the projector-augmented wave (PAW) method, ${ }^{4,5}$ which uses a radial grid projection from the atom center to map the core wave functions while mapping the valence wave functions with the plane-wave expansion. ${ }^{1}$ The set of planewaves used in DFT is determined through the optimization of a "cutoff energy" $\left(E_{\text {cut }}\right)$ which incorporates all valence electrons with kinetic energies at or below $E_{c u t}$. Electrons with kinetic energies greater than $E_{c u t}$ are assumed to be associated with the core and are therefore not considered. Convergence tests are used to determine this value. The PAW pseudopotential approach was determined to be the most accurate and useful method for this dissertation work. 


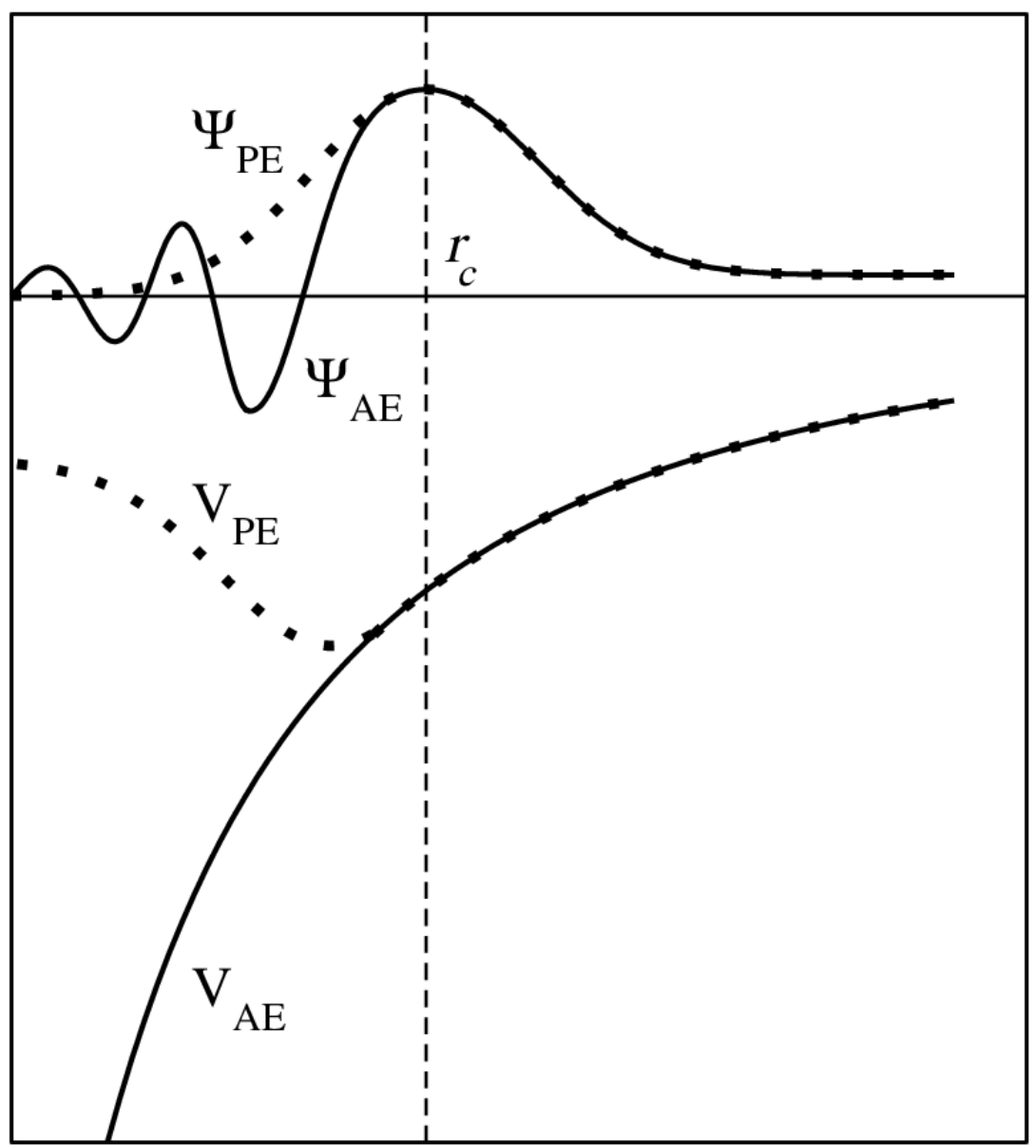

Figure 3.2. $\quad$ Schematic demonstrating the pseudo-electron (PE) (dotted lines) and all-electron (AE) (solid lines) wave function, $\Psi$, and potential, $V$. The PE and AE values must match at $r \geq r_{c}$ where $r_{c}$ represents the core radius.

The scf approach describes the method used to solve the KS equation and determine the final ground state energy. The approach, outlined in Figure 3.3, begins with rough estimates for the KS wave functions (taken from the pseudopotentials of each element) and uses them to calculate electron densities, which are used to calculate $\widehat{H}_{K S}$; and $\widehat{H}_{K S}$ is then used to generate new electron densities. This method is iterated until the input and output orbitals converge and "self-consistency" is reached. There are a number of parameters that govern the scf calculation. These include matrix diagonalization schemes, charge density mixing, and energy convergence thresholds. Once the first scf step is completed, the forces are calculated and atomic positions are updated to minimize 
the total energy of the system. Then, new scf steps are performed and the cycle is repeated until a predetermined force convergence threshold is reached and the effective ground state is determined (i.e., the structure is "relaxed"). Convergence tests were conducted to determine necessary parameters involved for relaxing these structures. 


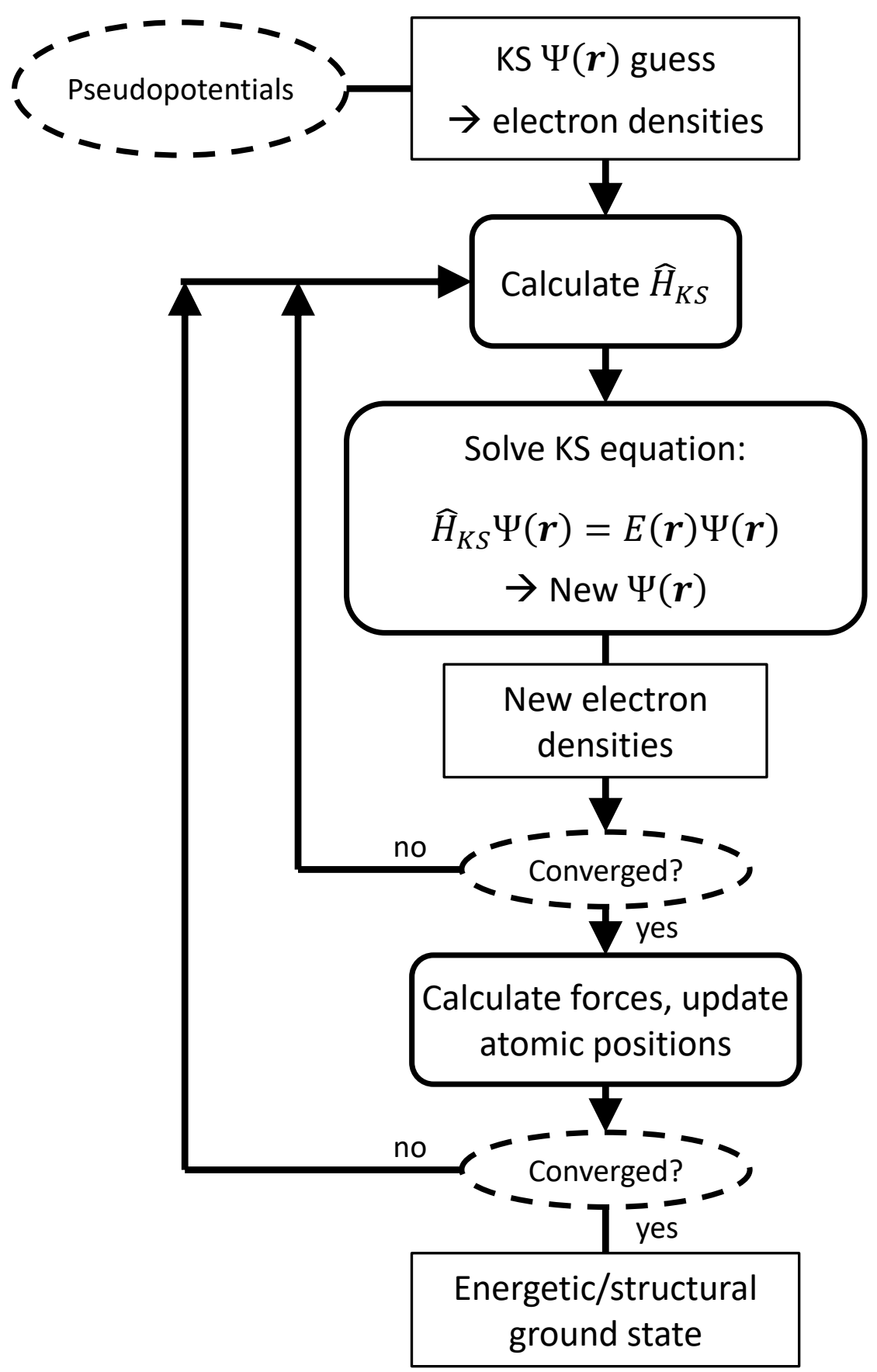

Figure 3.3. Flowchart describing the scf and structural relaxation method performed during a DFT calculation. A single scf step is completed when the first "convergence" step is reached. By calculating the forces after an scf step and adjusting the atomic positions, the forces can be minimized until a "relaxed" ground state configuration is determined. 


\subsection{Electrical Transport Properties}

After the ground state is determined for a given system, the electronic density of states (DoS) and energy band diagrams can be calculated; however, a known shortcoming of DFT is its tendency to underestimate and even eliminate bandgaps. This effect is especially present with strong localized d- and f-orbitals. A method for correcting this involves the use of DFT+U which adds an on-site Coulomb potential term (U) and an onsite exchange term $(\mathrm{J}){ }^{6}$ Though typically obtained semi-empirically, the application of these terms can greatly improve the accuracy of electronic structure calculations without increasing computational costs. As described in Figure 3.1, the optimized band energies can be used as input to Boltzmann transport property calculations. ${ }^{7}$

\section{Expansion of Band Energies}

The ground-state band energies of a material contain much information about various material properties. Madsen demonstrated that transport properties such as $\mathrm{S}, \sigma$, and the Hall coefficient $\left(R_{H}\right)$ can be calculated from the band energies. ${ }^{7}$ Through the use of star functions, the band energies can be expanded in Fourier space according to

$$
\begin{gathered}
\tilde{\varepsilon}_{i}(\boldsymbol{k})=\sum_{\boldsymbol{R}} c_{\boldsymbol{R}, i} S_{\boldsymbol{R}}(\boldsymbol{k}) \\
S_{\boldsymbol{R}}(\boldsymbol{k})=\frac{1}{n} \sum_{\{\Lambda\}} e^{i \boldsymbol{k} \cdot \Lambda \mathbf{R}}
\end{gathered}
$$

where $\mathbf{R}$ is the direct lattice vector and $\{\Lambda\}$ are the $n$ point group rotations.

The group velocity, $v_{\alpha}$, and inverse mass tensor, $M_{\beta u}^{-1}$, can be calculated from the first and second derivative of the band energies, respectively: 


$$
\begin{aligned}
& v_{\alpha}(i, \boldsymbol{k})=\frac{1}{\hbar} \frac{\partial \varepsilon_{i, \boldsymbol{k}}}{\partial k_{\alpha}} \\
& M_{\beta u}^{-1}(i, \boldsymbol{k})=\frac{1}{\hbar^{2}} \frac{\partial^{2} \varepsilon_{i, \boldsymbol{k}}}{\partial k_{\beta} \partial k_{u}}
\end{aligned}
$$

Here, $\hbar$ represents the reduced Planck's constant while the symbol $\varepsilon_{i, k}$ represents the energy of band $i$ at a given $\boldsymbol{k}$-point. Then the conductivity tensors can be found using

$$
\begin{aligned}
& \sigma_{\alpha \beta}(i, \boldsymbol{k})=e^{2} \tau_{i, \boldsymbol{k}} v_{\alpha}(i, \boldsymbol{k}) v_{\beta}(i, \boldsymbol{k}) \\
& \sigma_{\alpha \beta \gamma}(i, \boldsymbol{k})=e^{3} \tau_{i, \boldsymbol{k}}^{2} \epsilon_{\gamma u v} v_{\alpha}(i, \boldsymbol{k}) v_{v}(i, \boldsymbol{k}) M_{\beta u}^{-1}
\end{aligned}
$$

where the latter tensor is written with respect to the Levi-Civita symbol, $\epsilon_{\gamma u v}{ }^{8,9}$ These can be expressed as a function of band energy, $\varepsilon$

$$
\begin{gathered}
\sigma_{\alpha \beta}(\varepsilon)=\frac{1}{N} \sum_{i, \boldsymbol{k}} \sigma_{\alpha \beta}(i, \boldsymbol{k}) \frac{\delta\left(\varepsilon-\varepsilon_{i, \boldsymbol{k}}\right)}{d \varepsilon} \\
\sigma_{\alpha \beta \gamma}(\varepsilon)=\frac{1}{N} \sum_{i, \boldsymbol{k}} \sigma_{\alpha \beta \gamma}(i, \boldsymbol{k}) \frac{\delta\left(\varepsilon-\varepsilon_{i, \boldsymbol{k}}\right)}{d \varepsilon}
\end{gathered}
$$

with $N$ number of $\boldsymbol{k}$-points. The resulting transport tensors can be calculated as functions of temperature and chemical potential

$$
\begin{aligned}
& v_{\alpha \beta}(T ; \mu)=\frac{1}{e T \Omega} \int \sigma_{\alpha \beta}(\varepsilon)(\varepsilon-\mu)\left[-\frac{\partial f_{u}(T ; \varepsilon)}{\partial \varepsilon}\right] d \varepsilon \\
& \sigma_{\alpha \beta}(T ; \mu)=\frac{1}{\Omega} \int \sigma_{\alpha \beta}(\varepsilon)\left[-\frac{\partial f_{u}(T ; \varepsilon)}{\partial \varepsilon}\right] d \varepsilon \\
& \sigma_{\alpha \beta \gamma}(T ; \mu)=\frac{1}{\Omega} \int \sigma_{\alpha \beta \gamma}(\varepsilon)\left[-\frac{\partial f_{u}(T ; \varepsilon)}{\partial \varepsilon}\right] d \varepsilon
\end{aligned}
$$

along with the Seebeck coefficient

$$
S_{i j}=E_{i}\left(\nabla_{j} T\right)^{-1}=\left(\sigma^{-1}\right)_{\alpha i} v_{\alpha j}
$$

The electron relaxation time, $\tau_{i, \boldsymbol{k}}$, is an important metric for calculating the transport properties as it describes the average time between scattering events. In 
practice, this is a non-trivial variable to calculate. The total relaxation time, $\tau_{t}$, can be found using Matthiesen's rule

$$
\frac{1}{\tau_{t}}=\sum_{j} \frac{1}{\tau_{j}}
$$

where $\tau_{j}$ represents individual relaxation times from each different scattering mechanism. ${ }^{10}$ Rather than calculating this for each band and $\boldsymbol{k}$ direction, we will use a constant relaxation time in this work. This approximation is favorable because the relaxation time has been shown to be relatively independent of direction, even in strongly anisotropic systems. ${ }^{11,12}$

The electronic contribution to the thermal conductivity, $\kappa_{e}$, can also be written in terms of the conductivity distribution

$$
\kappa_{e, \alpha \beta}(T ; \mu)=\frac{1}{e^{2} T \Omega} \int \sigma_{\alpha \beta}(\varepsilon)(\varepsilon-\mu)^{2}\left[-\frac{\partial f_{u}(T ; \varepsilon)}{\partial \varepsilon}\right] d \varepsilon
$$

This term dominates the thermal conductivity for metals but is less impactful in semiconductors and insulators. For nonmetals, the thermal conductivity is dominated instead by the lattice thermal conductivity, $\kappa_{l}$, where

$$
\kappa=\kappa_{e}+\kappa_{l}
$$

To calculate $\kappa_{l}$, the phonon properties of the material must be investigated.

\subsection{Thermal Transport Properties}

In order to estimate the thermal transport properties of a material, it is necessary to analyze the phonon behavior. In general, the thermal conductivity is given in Equation 3.17 where $\kappa_{l}$ is the contribution from the lattice waves (phonons). For semiconductors, this term is expected to dominate as the contribution from electrons, $\kappa_{e}$, would be negligible. There are a few different methods for modeling phonon behavior; however, the phenomenon of heat conduction is a problem of multiphysics and multilength scales. 
Nevertheless, if one is drawing a comparison between similar systems, it is reasonable to identify trends via first-principles phonon property calculations. In this regard, DFT is often used as a calculator to estimate second- and third-order interatomic force constant matrices. These matrices are then used as input for phonon calculations which can be used to calculate phonon dispersion and DoS data (see Figure 3.1). Though third-order force constants are needed to account for Umklapp phonon scattering processes and determine $\kappa_{l}$, the second-order force constants can provide useful information about the phonon properties with only a fraction of the computational cost. For the screening purposes of this dissertation, only the second-order force constants are calculated to compare phonon property effects of dopants and heterostructures.

\section{$\underline{\text { Second-Order Force Constants }}$}

This work employs the method of DFPT to calculate the second-order force constants of a system. In this approach, an external perturbation is used to displace the atoms in order to determine the Hessian matrix. By defining the potential, $V$, of the phonon system as a function of atomic positions, the force, $F$, and second-order force constant, $\Phi_{\alpha \beta}$, are given as

$$
\begin{gathered}
F_{\alpha}(j l)=-\frac{\partial V}{\partial r_{\alpha}(j l)} \\
\Phi_{\alpha \beta}\left(j l, j^{\prime} l^{\prime}\right)=\frac{\partial^{2} V}{\partial r_{\alpha}(j l) \partial r_{\beta}\left(j^{\prime} l^{\prime}\right)}=-\frac{\partial F_{\beta}\left(j^{\prime} l^{\prime}\right)}{\partial r_{\alpha}(j l)}
\end{gathered}
$$

where the indices $\alpha$ and $\beta$ are Cartesian, $j$ and $j^{\prime}$ represent atoms in a unit cell, and $l$ and $l^{\prime}$ represent the unit cell. ${ }^{13}$

Through the implementation of the Phonopy software package, ${ }^{14}$ the force constants can be determined following the method of Parlinski, Li, and Kawazoe. ${ }^{15}$ In this approach, Equation 3.19 is given as the matrices, 


$$
\begin{aligned}
& \mathbf{F}=-\mathbf{U P} \\
& \mathbf{F}=\left(\begin{array}{l}
F_{x} \\
F_{y} \\
F_{z}
\end{array}\right) \\
& \mathbf{P}=\left(\begin{array}{c}
\Phi_{x x} \\
\Phi_{x y} \\
\Phi_{x z} \\
\Phi_{y x} \\
\Phi_{y y} \\
\Phi_{y z} \\
\Phi_{z x} \\
\Phi_{z y} \\
\Phi_{z z}
\end{array}\right) \\
& \mathbf{U}=\left(\begin{array}{ccccccccc}
\Delta r_{x} & 0 & 0 & \Delta r_{y} & 0 & 0 & \Delta r_{z} & 0 & 0 \\
0 & \Delta r_{x} & 0 & 0 & \Delta r_{y} & 0 & 0 & \Delta r_{z} & 0 \\
0 & 0 & \Delta r_{x} & 0 & 0 & \Delta r_{y} & 0 & 0 & \Delta r_{z}
\end{array}\right)
\end{aligned}
$$

where the $\mathbf{U}$ matrix represents atomic displacements. Through the use of $\mathbf{A}$ (9x9 sitepoint symmetry matrices) terms, the system can be solved by pseudo inverse via the following equation

$$
\left(\begin{array}{c}
\mathbf{F}_{1}^{(1)} \\
\mathbf{F}_{1}^{(2)} \\
\vdots \\
\mathbf{F}_{2}^{(1)} \\
\mathbf{F}_{2}^{(2)} \\
\vdots
\end{array}\right)=-\left(\begin{array}{c}
\mathbf{U}_{1} \mathbf{A}^{(1)} \\
\vdots \\
\mathbf{U}_{1} \mathbf{A}^{(2)} \\
\mathbf{U}_{2} \mathbf{A}^{(1)} \\
\mathbf{U}_{2} \mathbf{A}^{(2)} \\
\vdots
\end{array}\right) \mathbf{P}
$$

where the superscript numbers indicate the index of site-symmetry operations. ${ }^{13}$ Atomistic Green's Function (AGF) Method

Thermal transport across a boundary, such as the boundary between two 2D TMDs in a lateral heterostructure, is limited by the Kapitza, or thermal resistance of the interface, ${ }^{16}$ which determines the ratio of the boundary temperature difference to the interfacial heat flux. A higher thermal resistance corresponds to a greater temperature 
difference at the interface for a given heat flux and can be useful for thermal insulation. This phenomenon has also received attention for potential thermoelectric applications. ${ }^{17-}$ 20

In semiconductors and insulators, the interfacial heat flux consists of phonons which are transmitted at the interface in a manner analogous to conventional acoustic and optical wave transmission. The probability of each phonon propagating across the interface, given by its transmission coefficient, depends on the phonon dispersion of the constituent materials and the atomistic structure of the interface and varies between 0 and 1 because of impedance to its transmission from the abrupt change in crystallographic structure at the interface. The interfacial heat flux, $Q$, in the $x$-direction can be intuitively expressed as the sum of the total phonon population weighted by their transmission coefficients as well as their frequencies and group velocities in the direction of the flux, i.e., in a two-dimensional material,

$$
Q=\frac{1}{4 \pi^{2} d} \int_{B Z} d^{2} k \sum_{n=1}^{9} \hbar \omega_{n}\left(k_{x}, k_{y}\right) N\left(\omega_{n}, T\right) t_{n}\left(k_{x}, k_{y}\right) v_{n}\left(k_{x}, k_{y}\right)
$$

where the reciprocal $\left(k_{x}, k_{y}\right)$ space integral is taken over the entire Brillouin zone, and $d$, $\hbar, T$, and $n$ are the film thickness, reduced Planck constant, temperature, and phonon branch index, respectively. The variables $\omega_{n}, N, t_{n}$, and $v_{n}$ represent the phonon frequency, Bose-Einstein distribution function, transmission coefficient, and phonon group velocity in the direction of the flux, respectively. The expression in Equation 3.25 can be simplified to obtain the expression

$$
Q(T)=\frac{1}{4 \pi^{2} d} \int_{0}^{\infty} d \omega \hbar \omega N(\omega, T) \int_{-\pi / a}^{+\pi / a} d k_{y} \Xi\left(k_{y}, \omega\right)
$$

where $\Xi\left(k_{y}, \omega\right)$ is the Fourier $\left(k_{y}\right)$ component of the overall transmission function given by 


$$
\Theta(\omega)=\frac{1}{2 \pi} \int_{-\pi / a}^{+\pi / a} d k_{y} \Xi\left(k_{y}, \omega\right) .
$$

It is necessary to decompose the overall transmission function into its Fourier components because they can be computed piece-wise in a more computationally efficient fashion, especially if the interface has translational symmetry.

Although it is a straightforward exercise to calculate the phonon frequencies and group velocities, the computation of the transmission function $\Xi\left(k_{y}, \omega\right)$ is decidedly more involved and requires the application of a more sophisticated numerical technique such as the atomistic Green's function (AGF) method. ${ }^{21}$ The AGF method, developed from the nonequilibrium Green's function (NEGF) theory for electrons, describes the lattice dynamics of a finite body, corresponding to the interface region and couple to two semi-infinite thermal reservoirs commonly referred to as leads, and determines how an incoming phonon propagates across the interface region from one lead to the other. Conceptually, the leads define the available bulk phonon channels while the lattice structure of the interface region determines the transition between each pair of channels (in-coming and out-going).

The AGF for the interface region $G_{C}\left(k_{y}, \omega\right)$ can be written as

$$
G_{C}\left(k_{y}, \omega\right)=\lim _{\eta \rightarrow 0}\left[\omega^{2}+i \eta-H_{C}\left(k_{y}\right)-\Sigma_{L}\left(k_{y}\right)-\Sigma_{R}\left(k_{y}\right)\right]^{-1}
$$

where $H_{C}\left(k_{y}\right), \Sigma_{L}\left(k_{y}\right)$, and $\Sigma_{R}\left(k_{y}\right)$ are the mass-normalized force constant, left-lead self-energy, and right-lead self-energy matrices for the Fourier component $k_{y}$, respectively. The self-energy matrix for the left lead is given by

$$
\Sigma_{L}\left(k_{y}\right)=H_{C L} g_{L}(\omega) H_{C L}^{\dagger}
$$


where $H_{C L}$ is the mass-normalized force constant matrix representing the interaction between the left lead and the interface region, and $g_{L}(\omega)$ is the uncoupled surface Green's function for the left lead. The self-energy matrix for the right lead is similarly defined. The transmission function, $\Xi\left(k_{y}, \omega\right)$, which determines the total transmission for the given $\omega$ and $k_{y}$ is given by

$$
\Xi\left(k_{y}, \omega\right)=\operatorname{trace}\left[\Gamma_{L}\left(k_{y}\right) \mathrm{G}_{C}\left(k_{y}, \omega\right) \Gamma_{R}\left(k_{y}\right) \mathrm{G}_{C}\left(k_{y}, \omega\right)^{\dagger}\right] .
$$

In Equations 3.28 and 3.29, the force constant matrices are calculated with DFT. A separate set of force-constant matrices is calculated for each lead and also for the interface region. At each frequency, the total transmission function in Equation 3.27 can be combined with the Landauer formula ${ }^{22,23}$ to yield the heat current and thermal conductance of the interface.

Additional information on the distribution of the transmitted phonons can be obtained through the use of mode-matching ${ }^{24,25}$ combined with the AGF method to extract the transmission coefficients of individual phonon modes and identify the constituent phonon modes in the interfacial heat flux. ${ }^{26}$ This approach has already been used to analyze the lateral heterostructure of single-atomic layer graphene - hexagonal boron nitride $(h$ - $\mathrm{BN}){ }^{26,27}$ This dissertation similarly uses this newly developed approach to investigate the phonon transmission across the $2 \mathrm{D} \mathrm{MoS} 2-\mathrm{WS}_{2}$ heterostructure interface and to obtain insights into the microscopic physical processes underlying heat transfer at the interface. 


\section{References}

(1) Lee, J. G. Computational Materials Science; CRC Press Taylor and Francis Group: Boca Raton, FL, 2012.

(2) Kohn, W.; Sham, L. J. Self-Consistent Equations Including Exchange and Correlation Effects. Phys Rev 1965, 140, 1133.

(3) Perdew, J. P.; Burke, K.; Ernzerhof, M. Generalized gradient approximation made simple. Phys Rev Lett 1996, 77, 3865.

(4) Blöchl, P. E. Projector augmented-wave method. Phys Rev B 1994, 50, 17953.

(5) Kresse, G.; Joubert, D. From ultrasoft pseudopotentials to the projector augmented-wave method. Phys Rev B 1999, 59, 1758.

(6) Liechtenstein, A. I.; Anisimov, V. I.; Zaanen, J. Density-functional theory and strong interactions: Orbital ordering in Mott-Hubbard insulators. Phys Rev B 1995, 52, R5467.

(7) Madsen, G. K. H.; Singh, D. J. BoltzTraP. A code for calculating bandstructure dependent quantities. Computer Physics Communications 2006, 175, 67.

(8) Hurd, C. M. The Hall Effect in Metals and Alloys; Plenum Press: New York, 1972.

(9) Arfken, G. B.; Weber, H. J. Mathematical Methods for Physicists; Sixth ed.; Elsevier Academic Press: San Diego, 2005.

(10) Chen, G. Nanoscale Energy Transport and Conversion; Oxford University Press: New York, 2005.

(11) Schulz, W. W.; Allen, P. B.; Trivedi, N. Hall-Coefficient of Cubic Metals. Phys Rev B 1992, 45, 10886.

(12) Allen, P. B.; Pickett, W. E.; Krakauer, H. Anisotropic Normal-State Transport-Properties Predicted and Analyzed for High-Tc Oxide Superconductors. Phys Rev B 1988, 37, 7482. 
(13) Togo, A.; Release 1.6.2 ed. https://atztogo.github.io/phonopy/, 2012.

(14) Togo, A.; Tanaka, I. First principles phonon calculations in materials science. Scripta Mater 2015, 108, 1.

(15) Parlinski, K.; Li, Z. Q.; Kawazoe, Y. First-principles determination of the soft mode in cubic ZrO2. Phys Rev Lett 1997, 78, 4063.

(16) Swartz, E. T.; Pohl, R. O. Thermal-Boundary Resistance. Reviews of Modern Physics 1989, 61, 605.

(17) Cahill, D. G.; Ford, W. K.; Goodson, K. E.; Mahan, G. D.; Majumdar, A.; Maris, H. J.; Merlin, R.; Sr, P. Nanoscale thermal transport. J Appl Phys 2003, 93, 793.

(18) Pop, E. Energy Dissipation and Transport in Nanoscale Devices. Nano Res 2010, 3, 147.

(19) Chen, P. X.; Katcho, N. A.; Feser, J. P.; Li, W.; Glaser, M.; Schmidt, O. G.; Cahill, D. G.; Mingo, N.; Rastelli, A. Role of Surface-Segregation-Driven Intermixing on the Thermal Transport through Planar Si/Ge Superlattices. Phys Rev Lett 2013, 111, 115901.

(20) Cahill, D. G.; Braun, P. V.; Chen, G.; Clarke, D. R.; Fan, S. H.; Goodson, K. E.; Keblinski, P.; King, W. P.; Mahan, G. D.; Majumdar, A.; Maris, H. J.; Phillpot, S. R.; Pop, E.; Shi, L. Nanoscale thermal transport. II. 2003-2012. Appl Phys Rev 2014, 1 .

(21) Mingo, N.; Yang, L. Phonon transport in nanowires coated with an amorphous material: An atomistic Green's function approach. Phys Rev B 2003, $68,245406$.

(22) Zhang, W.; Fisher, T. S.; Mingo, N. The atomistic Green's function method: An efficient simulation approach for nanoscale phonon transport. Numer Heat Tr B-Fund 2007, 51, 333.

(23) Landauer, R. Conductance Determined by Transmission - Probes and Quantized Constriction Resistance. J Phys-Condens Mat 1989, 1, 8099. 
(24) Khomyakov, P. A.; Brocks, G.; Karpan, V.; Zwierzycki, M.; Kelly, P. J. Conductance calculations for quantum wires and interfaces: Mode matching and Green's functions. Phys Rev B 2005, 72, 035450.

(25) Wang, J. S.; Wang, J.; Lu, J. T. Quantum thermal transport in nanostructures. Eur Phys J B 2008, 62, 381.

(26) Ong, Z. Y.; Zhang, G. Efficient approach for modeling phonon transmission probability in nanoscale interfacial thermal transport. Phys Rev $B$ 2015, $91,174302$.

(27) Ong, Z. Y.; Zhang, G.; Zhang, Y. W. Controlling the thermal conductance of graphene/h-BN lateral interface with strain and structure engineering. Phys Rev B 2016, 93, 075406. 


\section{CHAPTER FOUR: IMPROVED THERMOELECTRIC PERFORMANCE OF (Fe,Co)Sb3-TYPE SKUTTERUDITES FROM FIRST-PRINCIPLES*}

This chapter is published by AIP Publishing in the Journal of Applied Physics and should be referenced appropriately.

Reference:

I. Williamson, L. J.-Y. Her, X. Su, Y. Yan, W. Wong-Ng, L. Li, “Improved thermoelectric performance of $(\mathrm{Fe}, \mathrm{Co}) \mathrm{Sb}_{3}$-type skutterudites from first-principles." Journal of Applied Physics. 119055101 (2016).

Reproduced from Journal of Applied Physics, 119055101 (2016), with the permission of AIP Publishing.

*This chapter includes modifications from the originally published version. 
Improved thermoelectric performance of $(\mathrm{Fe}, \mathrm{Co}) \mathrm{Sb}_{3}$-type skutterudites from firstprinciples

\author{
Izaak Williamson $^{\mathrm{a}}$ \\ Logan Ju-Yee Her ${ }^{\mathrm{b}}$ \\ Xianli $\mathrm{Su}^{\mathrm{c}}$ \\ Yonggao $\mathrm{Yan}^{\mathrm{c}}$ \\ Winnie Wong-Ng ${ }^{\mathrm{d}}$ \\ Lan $\mathrm{Li}^{\mathrm{a}, \mathrm{e}}$
}

\begin{abstract}
Published in:
Journal of Applied Physics

January 2016

${ }^{a}$ Department of Materials Science and Engineering, Boise State University, Boise, ID 83725 .

${ }^{b}$ Department of Science, Math, and Engineering, Cosumnes River College, Sacramento, CA 95823.
\end{abstract}

${ }^{c}$ State Key Laboratory of Advanced Technology for Materials Synthesis and Processing, Wuhan University of Technology, Wuhan, Hubei 430070, China. 
${ }^{d}$ Materials Measurement Science Division, National Institute of Standards and Technology,

Gaithersburg, MD 20899.

${ }^{e}$ Center for Advanced Energy Studies,

Idaho Falls, ID 83401. 


\begin{abstract}
Skutterudite materials have been considered as promising thermoelectric candidates due to intrinsically good electrical conductivity and tailorable thermal conductivity. Options for improving thermal-to-electrical conversion efficiency include identifying novel materials, adding filler atoms, and substitutional dopants. Incorporating filler or substitutional dopant atoms in the skutterudite compounds can enhance phonon scattering, resulting in reduction of thermal conductivity, as well as improving electrical conductivity. The structures, electronic properties, and thermal properties of double-filled $\mathrm{Ca}_{0.5} \mathrm{Ce}_{0.5} \mathrm{Fe}_{4} \mathrm{Sb}_{12}$ and $\mathrm{Co}_{4} \mathrm{Sb}_{12-2 \mathrm{x}} \mathrm{TexGe}_{\mathrm{x}}$ compounds $(x=0,0.5,1,2,3$, and 6$)$ have been studied using density functional theory (DFT) based calculations. Both $\mathrm{Ca} / \mathrm{Ce}$ filler atoms in $\mathrm{FeSb}_{3}$ and $\mathrm{Te} / \mathrm{Ge}$ substitution in $\mathrm{CoSb}_{3}$ cause a decrease in lattice constant for the compounds. As Te/Ge substitution concentration increases, lattice constant decreases and structural distortion of pnictogen rings in the compounds occurs. This indicates a break in cubic symmetry of the structure. The presence of fillers and substitutions causes an increase in electrical conductivity and a decrease in electronic bandgap. A transition from direct to indirect band-gap semiconducting behavior is found at $x=3$. Phonon density of states for both compounds indicate phonon band broadening by the incorporation of fillers and substitutional atoms. The acoustic phonon modes are assumed to dominate thermal transport for both systems. For the $\mathrm{Co}_{4} \mathrm{Sb}_{12-2 \mathrm{x}} \mathrm{Te}_{\mathrm{x}} \mathrm{Ge}_{\mathrm{x}}$ compounds, $x=3$ exhibits the lowest phonon dispersion gradient and lattice thermal conductivity, agreeing well with experimental measurements. Our results present the improvement of thermoelectric properties of skutterudite compounds through fillers and substitutional doping.
\end{abstract}




\subsection{Introduction}

The combustion of fuel in automobiles is strikingly inefficient where approximately $75 \%$ of energy produced during this process is lost as waste heat. Thermoelectric materials can be used to recover some of this waste heat by converting it to useful electrical power for the vehicle. The performance of these materials is determined by a dimensionless figure of merit $Z T$ (see Equation 2.1). Materials with higher $Z T$ values $(Z T>1)$ have greater thermoelectric performance. Such materials must exhibit a high power factor while having low thermal conductivity. In order to meet these criteria, it is important to identify novel materials or substitute existing materials with different species to optimize the $Z T$ value. ${ }^{1-11}$

One class of materials that has potential for thermoelectric applications are skutterudite compounds. These are relatively low-cost and easy to process materials that intrinsically exhibit good electrical transport properties and tunable thermal transport properties with site substitutions. ${ }^{2,5,9-16}$ Skutterudites have the space group $\operatorname{Im} 3$ and consist of cage-like structures with the general formula $\mathrm{AB}_{3}$ where $\mathrm{A}$ is a transition metal and $\mathrm{B}$ is a pnictogen. Figure 2.1 shows the crystal structure of a filled skutterudite featuring $\mathrm{M}$ filler atoms and $\mathrm{B}$ rings within an A sublattice, where $\mathrm{M}=\mathrm{La}, \mathrm{Te}, \mathrm{Ge}, \mathrm{Ba}$, In, or $\mathrm{Yb} ; \mathrm{A}=\mathrm{Fe}, \mathrm{Ru}, \mathrm{Co}, \mathrm{Ni}$, or Os; $\mathrm{B}=\mathrm{Sb}, \mathrm{P}$, or As. Skutterudite compounds generally have a $Z T$ value around 1 and maintain large carrier concentrations and moderate Seebeck coefficients. ${ }^{17,18}$ The incorporation of filler atoms reduces their thermal conductivity through the "rattling motion." Alkaline earths have previously been used as fillers ${ }^{5}$ and Yang et al. ${ }^{19}$ have shown that a double-filled skutterudite, having one alkaline earth and one lanthanide $(\mathrm{Ba}$ and $\mathrm{Ce}$ ) filler, is even more effective at reducing the lattice 
thermal conductivity than using two alkaline earths ( $\mathrm{Ba}$ and $\mathrm{Sr}$ ). The combination of $\mathrm{Ca}$ and $\mathrm{Ce}$ used in this report mimics this approach but for elements with greater mass difference (71\% mass difference compared to just $2 \%)$. The structure of a skutterudite also has large degrees of freedom for doping and alloying. This makes it attractive for customization and design studies.

In addition to incorporating filler atoms, substituting B sites with different species is another approach to improve the $Z T$ value. Cobalt triantimonide $\left(\mathrm{CoSb}_{3}\right)$ is a skutterudite of particular interest for power generation because it can operate at high temperatures $(\approx 900 \mathrm{~K})$. Substitutions that have been investigated for the $\mathrm{CoSb}_{3}$ structure include $\mathrm{Cr},{ }^{20} \mathrm{Ni},{ }^{21}$ or $\mathrm{Fe},{ }^{22}$ on the Co site, and $\mathrm{Ge}$ or Te on the Sb site. These substitutions reveal significant changes in transport properties such as an increase in electrical conductivity and a decrease in thermal conductivity; therefore, a fundamental investigation of fillers and substitutions and their effects on the thermoelectric properties of skutterudite compounds is important for materials development.

As a follow-up to our previous work, ${ }^{22}$ this paper investigates the effect of $\mathrm{Ca} / \mathrm{Ce}$ filler atoms in $\mathrm{FeSb}_{3}$ and $\mathrm{Te} / \mathrm{Ge}$ substitutions on the $\mathrm{Sb}$ site in $\mathrm{CoSb}_{3}$. The stoichiometry of the $\mathrm{Ca} / \mathrm{Ce}$ double-filled system follows the form $\mathrm{CaCeFe}_{8} \mathrm{Sb}_{24}$ but the proper ordering of the $\mathrm{Ca}$ and $\mathrm{Ce}$ atoms follows that of Figure 4.1 which requires a supercell of $\mathrm{Ca}_{4} \mathrm{Ce}_{4} \mathrm{Fe}_{32} \mathrm{Sb}_{96}$ to describe the system. In $\mathrm{Co}_{4} \mathrm{Sb}_{12-2 \mathrm{x}} \mathrm{Te}_{\mathrm{x}} \mathrm{Ge}_{\mathrm{x}}$, six compositions are considered where $\mathrm{x}=0,0.5,1,2,3$, and 6 . This range of compositions offers a complete look at the substitution up to a complete replacement of the $\mathrm{Sb}$ atoms $\left(\mathrm{Co}_{4} \mathrm{Te}_{6} \mathrm{Ge}_{6}\right)$. To investigate the atomic structure features, we calculated the lattice constant, $a$, for the $\mathrm{Ca}_{0.5} \mathrm{Ce}_{0.5} \mathrm{Fe}_{4} \mathrm{Sb}_{12}$ system and lattice constant, $a$, and cell angle, $\gamma$, as a function of $x$ for 
the $\mathrm{Co}_{4} \mathrm{Sb}_{12-2 \mathrm{x}} \mathrm{Te}_{\mathrm{x}} \mathrm{Ge}_{\mathrm{x}}$ system. The atomic, electronic, and phonon dispersion effects were also investigated for each compound. This work helps identify the (Fe,Co)Sb$b_{3}$-type compounds with a stable structure and optimized electrical and thermal conductivity properties, hence improved $Z T$ values.

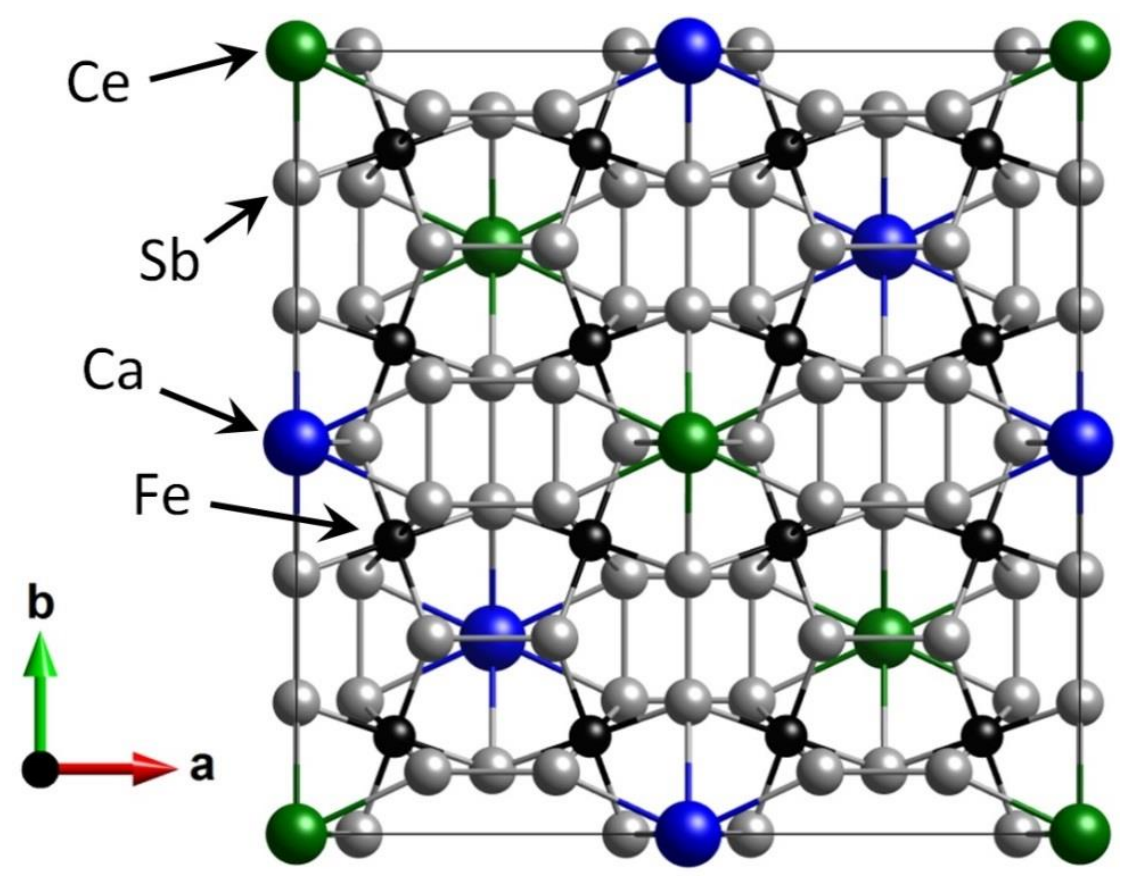

Figure 4.1. Schematic of the $\mathrm{Ca}_{4} \mathrm{Ce}_{4} \mathrm{Fe}_{32} \mathrm{Sb}_{96}$ crystal structure where $\mathrm{Ca}$ (blue), Ce (green), Fe (black), and $\mathrm{Sb}$ (gray) atoms are represented by spheres. The preferred ordering of the filler atoms is to arrange all $\mathrm{Ca}$ atoms along (220) planes and all $\mathrm{Ce}$ atoms along (110) planes in this $4 \times 4 \times 2$ supercell.

\subsection{Computational Methods}

Crystal structure and electronic structure calculations were performed within the DFT through implementation of the VASP code. ${ }^{23}$ The spin-dependent GGA functional was used with the Perdew-Burke-Ernzerhof (PBE) formalism. ${ }^{24}$ Projector augmented wave (PAW) pseudopotentials were employed with a plane wave expansion cutoff of 500 $\mathrm{eV}$ and a $6 \times 6 \times 6$-centered $k$-point mesh for Brillouin zone integration. ${ }^{25,26}$ Fermi surface 
broadening was accounted for by a Gaussian smearing of $0.05 \mathrm{eV}$ for the $\mathrm{FeSb}_{3}$ structures while $0.025 \mathrm{eV}$ was used for the $\mathrm{CoSb}_{3}$ structures. Atomic positions and lattice vectors were relaxed until the residual forces were reduced to less than $0.01 \mathrm{eV} / \AA$. Electronic structure calculations, including DoS and band structure calculations, were carried out on the DFT-optimized structures. A larger $k$-point mesh of $12 \times 12 \times 12$ was used for the Brillouin zone integration. These calculations were performed in order to investigate the effects of $\mathrm{Ca} / \mathrm{Ce}$ fillers and $\mathrm{Te} / \mathrm{Ge}-\mathrm{Sb}$ substitution on the electrical conductivity of the systems. Strong correlation effects were also tested via the DFT+U scheme for the Fe atoms and Co atoms (on-site Coulomb potential $\mathrm{U}_{\mathrm{Fe}}=4.5 \mathrm{eV}$ and $\mathrm{U}_{\mathrm{Co}}=7.8 \mathrm{eV}$; exchange potential $\mathrm{J}_{\mathrm{Fe}}=0.89 \mathrm{eV}$ and $\left.\mathrm{J}_{\mathrm{Co}}=0.92 \mathrm{eV}\right) .{ }^{27}$ This approach is often used to correct the bandgap which is underestimated with DFT alone.

To estimate the thermal properties of each system, DFPT was used to calculate the necessary force constants by means of the Parlinski-Li-Kawazoe method. ${ }^{28}$ The phonon density of states and phonon dispersion relations were then generated using the PHONOPY software package. ${ }^{29}$ Force constant calculations were performed using an energy cutoff of $400 \mathrm{eV}$ and a $k$-point mesh of $1 \mathrm{x} 1 \mathrm{x} 1$ for each structure. To account for long-range phonon interactions, a supercell convergence test was performed for each of the $\mathrm{Co}_{4} \mathrm{Sb}_{12-2 \mathrm{x}} \mathrm{Te}_{\mathrm{x}} \mathrm{Ge}_{\mathrm{x}}$ structures.

The use of these methods and input parameters comes from optimization tests which balance system accuracy with computation costs. For most parameters, this point is reached when a further reduction in total system energy is negligible yet computation time is greatly increased. Once the DFT environment has been optimized, resulting lattice 
constants and cell volume data were compared with experimental results to ensure accuracy in the models.

\subsection{Results}

\section{$\underline{\text { Structural Effects }}$}

The $\mathrm{FeSb}_{3}$ skutterudite has a cubic crystal structure with the $\operatorname{Im} 3$ space group and consists of $\mathrm{Fe}$ and $\mathrm{Sb}$ at $\mathrm{A}$ and B sites, respectively (Figure 2.1). It has a cage-like structure with two icosahedral voids filled with guest atoms M. Previous work indicated that $\mathrm{Ca}$ and $\mathrm{Ce}$ atoms as fillers with a 1:1 ratio form $\mathrm{Ca}_{0.5} \mathrm{Ce}_{0.5} \mathrm{Fe}_{4} \mathrm{Sb}_{12}$ and exhibit a higher $Z T$ value than that of other $\left(\mathrm{Ca}_{\mathrm{x}} \mathrm{Ce}_{1-\mathrm{x}}\right) \mathrm{Fe}_{4} \mathrm{Sb}_{12}$ compounds where $x=0,0.25,0.75$, and $1 .{ }^{22}$ This is the consequence of large mass difference between $\mathrm{Ca}$ and $\mathrm{Ce}$, generating a wider range of resonant rattling frequencies and leading to phonon scattering enhancement and thermal conductivity decrease.

This work further studies the $\mathrm{Ca}_{0.5} \mathrm{Ce}_{0.5} \mathrm{Fe}_{4} \mathrm{Sb}_{12}$ compound. DFT structural calculations indicate a decrease in lattice constant from $9.1805 \AA$ for the unfilled compound to $9.1777 \AA$ ( $~ 0.03 \%)$ for the double-filled compound, which agrees well with the experimental lattice constant of $9.149056 \AA$ for $\mathrm{Ca}_{0.5} \mathrm{Ce}_{0.5} \mathrm{Fe}_{4} \mathrm{Sb}_{12}$. As shown in Figure 4.1, a large 136 atom $4 \times 4 \times 2$ supercell (i.e., $\mathrm{Ca}_{4} \mathrm{Ce}_{4} \mathrm{Fe}_{32} \mathrm{Sb}_{96}$ ) was used where the $\mathrm{Ca}$ and Ce were arranged with each occupying alternating (110) planes (defined using the $2 \times 2 \times 2$ supercell). The structure has average $\mathrm{Ca}-\mathrm{Sb}$ and $\mathrm{Ce}-\mathrm{Sb}$ bond lengths of $3.353 \AA$ and $3.361 \AA$, respectively; $\mathrm{Sb}-\mathrm{Ca}-\mathrm{Sb}$ bond angles of $52.00^{\circ}$ and $66.85^{\circ}$; and $\mathrm{Sb}-\mathrm{Ce}-\mathrm{Sb}$ bond angles of $52.09^{\circ}$ and $66.82^{\circ}$.

The effects of B-site substitution in skutterudites were also investigated using the $\mathrm{CoSb}_{3}$ system where two $\mathrm{Sb}$ atoms are incrementally replaced with $\mathrm{Te}$ and Ge atoms, 
resulting in the formula $\mathrm{Co}_{4} \mathrm{Sb}_{12-2 \mathrm{x}} \mathrm{Te}_{\mathrm{x}} \mathrm{Ge}_{\mathrm{x}}$, where $x=0,0.5,1,2,3$, and 6. Our optimized crystal structures and calculated lattice constants, $a$, were compared with experimental values, determined by the Rietveld refinement technique and a Rietveld pattern decomposition technique. ${ }^{30,31}$ Table 4.1 shows the calculated and experimental lattice parameters for $\mathrm{Co}_{4} \mathrm{Sb}_{12-2 \mathrm{x}} \mathrm{Te}_{\mathrm{x}} \mathrm{Ge}_{\mathrm{x}}$ to illustrate the structural effects of B-site substitutions Te/Ge. The lattice constant $a$ appears to decrease almost linearly as $x$ increases, with the exception of $x=2$. These results are expected, given that the covalent radius of $\mathrm{Sb}(1.40$ $\AA)$ is larger than that of the substituents Te $(1.36 \AA)$ and Ge $(1.21 \AA) .{ }^{32}$ Cell angle $\gamma$ slightly shrinks at $x=0.5,1$, and 3 , but it enlarges at $x=6$. These changes indicate that the symmetry of the $\mathrm{Co}_{4} \mathrm{Sb}_{12-2 \mathrm{x}} \mathrm{Te}_{\mathrm{x}} \mathrm{Ge}_{\mathrm{x}}$ compound is no longer cubic with $\mathrm{Te} / \mathrm{Ge}$ substitution. Our DFT results agree well with experimental XRD structural analysis results $(<1.0 \%$ error $)$.

Table 4.1 Calculated cell angle $\gamma$ in degrees and lattice parameter $\boldsymbol{a}$ in units of $\AA$ for $\mathrm{Co}_{4} \mathrm{Sb}_{12-2 \mathrm{x}} \mathrm{Te}_{\mathrm{x}} \mathrm{Ge}_{\mathrm{x}}(x=0,0.5,1,2,3$, and 6$)$. The experimentally determined lattice parameter $a_{\text {exp }}$ and the percent error between calculated and experimental values is also given (subscript $\exp$ denotes experimental values). ${ }^{31}$ The symbol $\theta$ represents the smallest distortion angle in the pnictogen rings. Superscript ${ }^{\text {a }}$ denotes values taken for the structure with the highest dopant site order (configuration 3).

\begin{tabular}{|c|c|c|c|c|c|}
\hline $\mathrm{x}$ & $\gamma\left(^{\circ}\right)$ & $a(\AA)$ & $a_{\exp }(\AA)$ & Error $\%$ & $\theta\left(^{\circ}\right)$ \\
\hline 0 & 90.00 & 9.11086 & 9.03662 & 0.82 & 90.00 \\
\hline 0.5 & 89.90 & 9.08122 & 9.01633 & 0.72 & 88.72 \\
\hline 1 & 89.83 & 9.06154 & 8.99555 & 0.73 & 85.62 \\
\hline 2 & 90.00 & 8.88705 & 8.94781 & -0.68 & 86.40 \\
\hline 3 & 89.96 & 8.96417 & 8.89584 & 0.77 & $80.87^{\mathrm{a}}$ \\
\hline 6 & 90.26 & 8.79712 & -- & -- & $81.84^{\mathrm{a}}$ \\
\hline
\end{tabular}


In order to better understand the atomic structures, we measured the distances between $\mathrm{Co}$ atoms and the rings consisting of $\mathrm{Sb} / \mathrm{Te} / \mathrm{Ge}$. In experiments, the Co$\mathrm{Sb} / \mathrm{Te} / \mathrm{Ge}$ distance decreases from $2.53 \AA$ to $2.49 \AA$ as $x$ increases from 0 to 3 . While the average $\mathrm{Sb} / \mathrm{Te} / \mathrm{Ge}$-Sb/Te/Ge distances in the 4-member rings also decreases from $2.91 \AA$ to $2.86 \AA$, they are longer than the typical Sb-Sb distance of $2.80 \AA{ }^{32}$ DFT calculations reveal that the average $\mathrm{Co}-\mathrm{Sb} / \mathrm{Te} / \mathrm{Ge}$ bond distances decrease from $2.54 \AA$ to $2.47 \AA$ from $x=0$ to 3 and even further decreased to $2.41 \AA$ at $x=6$, which is consistent with experimental results. From $x=0.5$ to 2 , the Co-Ge bond distance is the shortest compared to the $\mathrm{Co}-\mathrm{Sb}$ and $\mathrm{Co}-\mathrm{Te}$ bonds; however, in the $x=3$ compound, the Co-Te bond distance decreases rapidly and becomes the shortest. Te/Ge substitution at $x=3$ results in a unique structure. Due to different $\mathrm{Co}-\mathrm{Sb} / \mathrm{Te} / \mathrm{Ge}$ bond distances and large $\mathrm{Te} / \mathrm{Ge}$ substitution concentration, the 4-member rings consisting of $\mathrm{Sb} / \mathrm{Te} / \mathrm{Ge}$ are distorted, and turn into the parallelogram rings shown in Figure 4.2. The distortions depend on the concentration of $\mathrm{Te} / \mathrm{Ge}$ substitution in the rings. Its angles range from $81^{\circ}$ to $93^{\circ}$.
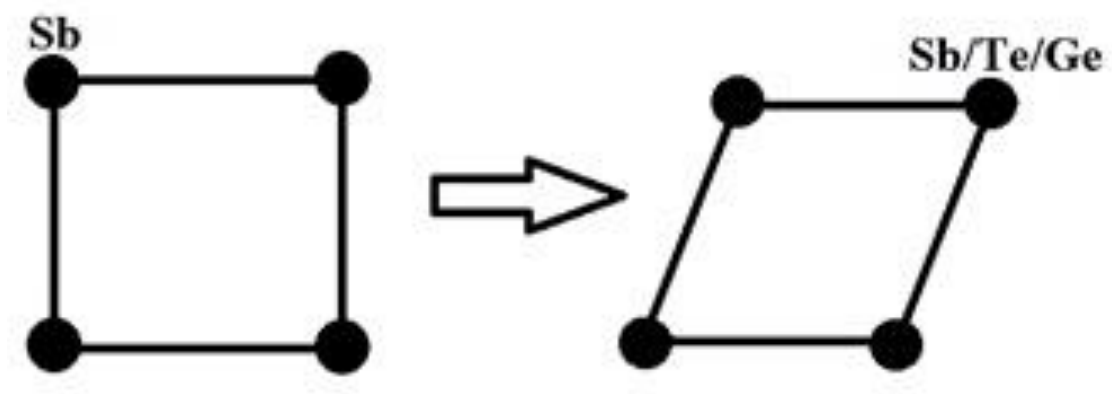

Figure 4.2. Model depicting pnictogen rings within the filled skutterudite structure. Upon $\mathrm{Te} / \mathrm{Ge}$ substitution, the ring distorts to an angle of down to $81^{\circ}$ for $\mathrm{Co4Sb}_{12-2 \mathrm{x}} \mathrm{Te}_{\mathrm{x}} \mathrm{Ge}_{\mathrm{x}}$ at $x=3$.

\section{Electrical Properties}

The $\mathrm{Ca}$ and $\mathrm{Ce}$ filler atoms in $\mathrm{FeSb}_{3}$ affect the electronic structure as well as the crystal structure. As shown in Figure 4.3, the electronic DoS for the $\mathrm{Fe}_{8} \mathrm{Sb}_{24}$ structure 
shows that the material is already electronically conductive because, at the Fermi level, there is low DoS for the spin-up (positive y-values) and relatively large DoS for the spindown. The difference in spin-up and spin-down DoS is caused by the magnetism of Fe in the system. The $\mathrm{Ca} / \mathrm{Ce}$-filled structure, having the proper $\mathrm{Ca}-\mathrm{Ce}$ ordering as shown in Figure 4.1, is also electronically conductive but with a more subtle difference between spin-up and spin-down DoS than the unfilled structure. Experimentally, it is shown that this particular $\mathrm{Ca}-\mathrm{Ce}$ ordered structure is not necessarily the most electronically conductive type of $\mathrm{Ca} / \mathrm{Ce}$-filled $\mathrm{FeSb}_{3}$ but is conductive enough to allow for high $\mathrm{ZT}$ values. ${ }^{22}$ Indeed, in Figure 4.3 the presence of a zero-DoS point near the Fermi level could indicate potential for this material to exhibit semi-metallic behavior.

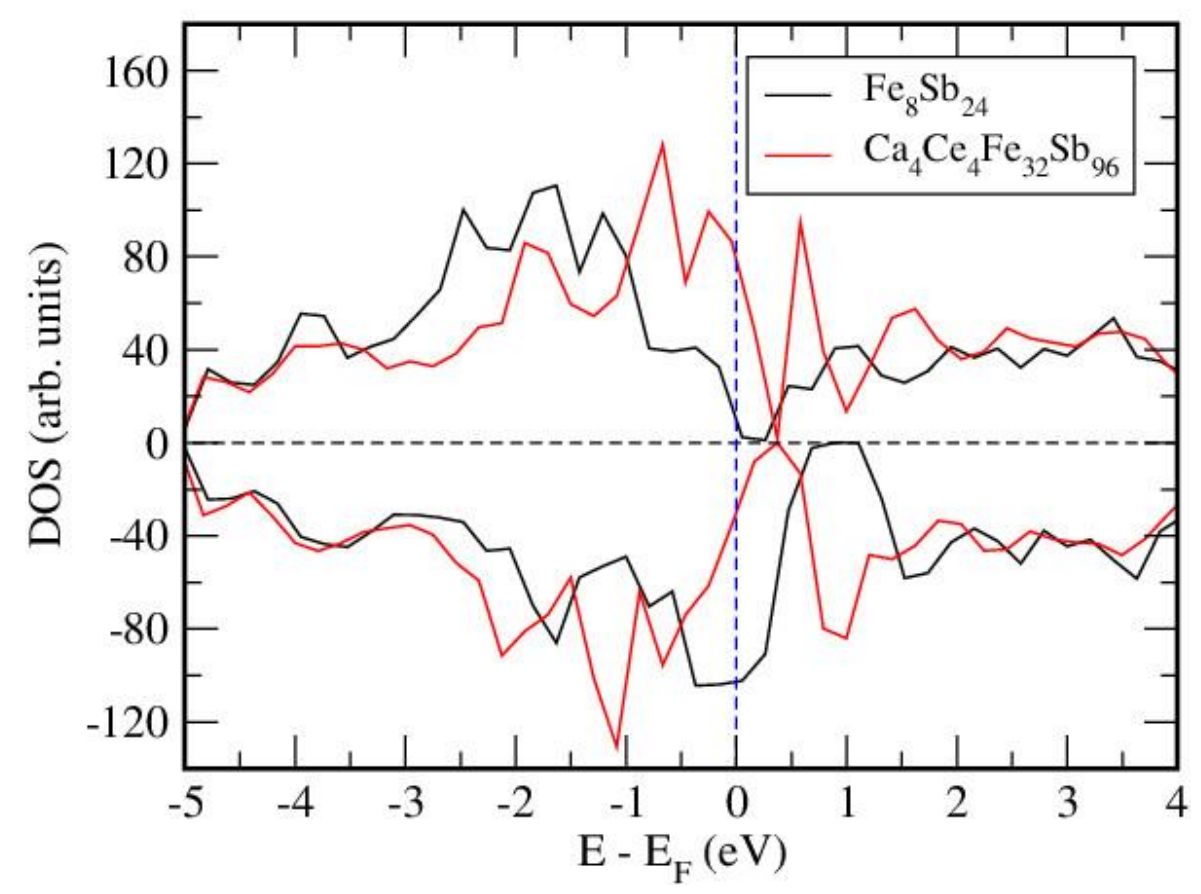

Figure 4.3. Electronic density of states for the unfilled (black line) and CaCe-filled (red line) FeSb3 structure. In order to account for the correct $\mathrm{Ca}-\mathrm{Ce}$ ordering as shown in Figure 4.1, a large $\mathrm{Ca}_{4} \mathrm{Ce}_{4} \mathrm{Fe}_{32} \mathrm{Sb96}$ supercell structure was used. For the sake of comparison, the DoS magnitude for the unfilled structure is increased by a factor of four. Dotted lines depict the zero values on both axes and the $x$-axis is shifted so the zero-point represents the Fermi level (depicted by the blue dotted line). The up- and down-spin DoS are given by positive and negative values, respectively. The difference 
in shape between spin-up and spin-down is primarily caused by the magnetism of the $\mathrm{Fe}$ atoms in the system. The unfilled $\mathrm{Fe}_{8} \mathrm{Sb}_{24}$ (black) results show a bandgap only for the positive DoS while neither the up- nor down-spin CaCe-filled (red) DoS has a significant bandgap.

Similarly, the electronic conductivity for the $\mathrm{CoSb}_{3}$ structure is affected by the concentration of $\mathrm{Te} / \mathrm{Ge}$ substitution. Through electronic structure calculations, we studied the Te/Ge substitution effect on the bandgap of each $\mathrm{Co}_{4} \mathrm{Sb}_{12-2 x} \mathrm{Te}_{\mathrm{x}} \mathrm{Ge}_{\mathrm{x}}$ compound $(x=0$, $0.5,1,2$, and 3), which allowed us to predict the electrical conductivity change. Comparison with experimental data ${ }^{30}$ validated the computational prediction. Further studies of local density of states provided insight into Co-Te/Ge bonding.

Figure 4.4a shows DoS for $\mathrm{Co}_{4} \mathrm{Sb}_{12-2 \mathrm{x}} \mathrm{Te}_{\mathrm{x}} \mathrm{Ge}_{\mathrm{x}}$. Fermi energy is shifted to $0 \mathrm{eV}$. At $x=0$, the compound is semiconducting with a bandgap of $\sim 0.16 \mathrm{eV}$. As $x$ increases to 0.5 , the bandgap decreases. Interestingly, when Te/Ge concentration continues increasing, the bandgap first opens wider but then completely closes at $x=3$. Such bandgap changes reveal the effect of $\mathrm{Te} / \mathrm{Ge}$ substitution on the electrical conductivity of the compound. The compound at $x=0$ is expected to have the smallest electrical conductivity while the highest electrical conductivity occurs at $x=3$ due to the absence of a bandgap. From $x=0.5$ to 1 , the electrical conductivity should slightly decrease due to the wider bandgap. These computational predictions are consistent with experimental measurements which show that increasing Te concentration in Ge-doped $\mathrm{CoSb}_{3}$ increases the electrical conductivity. ${ }^{33,34}$ 
(a)

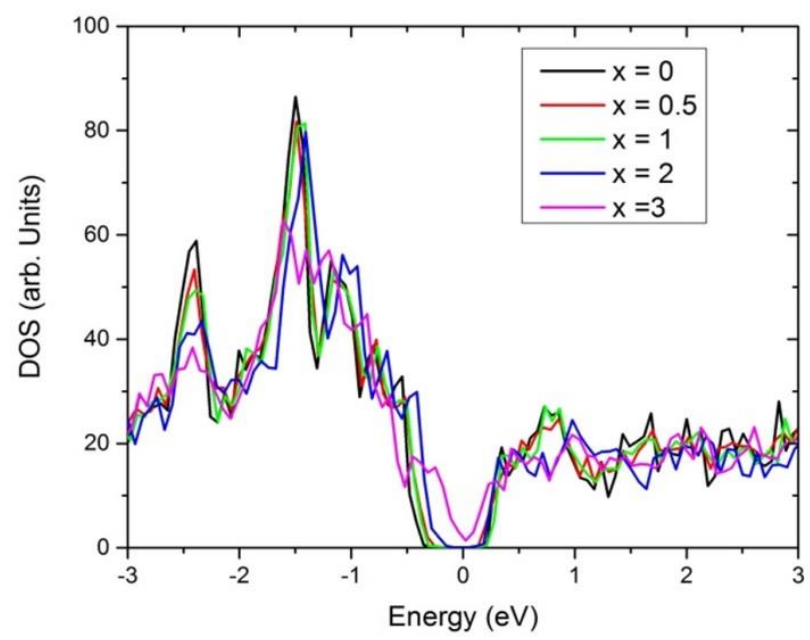

(b)

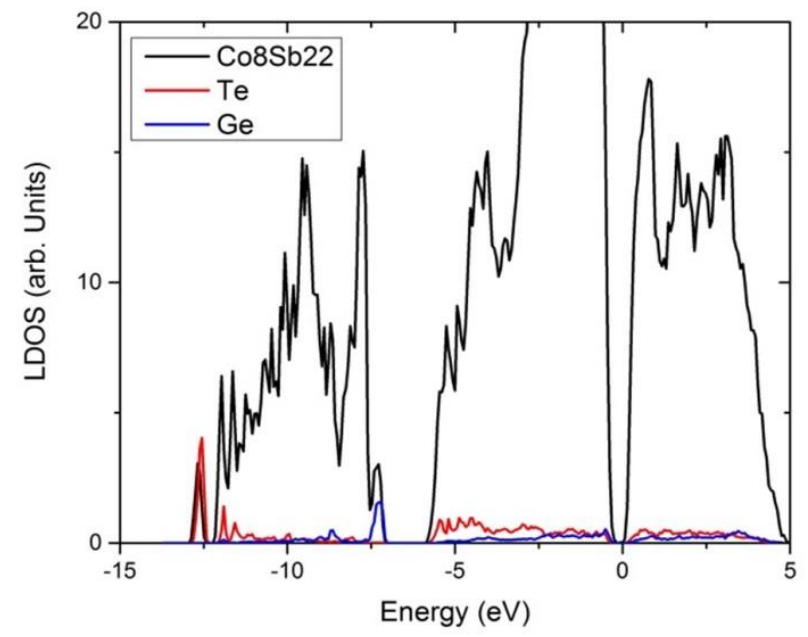

Figure 4.4. Electronic density of states for $\mathrm{Co}_{4} \mathrm{Sb}_{12-2 x} \mathrm{Te}_{\mathrm{x}} \mathrm{Ge}_{\mathrm{x}}$, Fermi energy $=\mathrm{O} \mathrm{eV}$ : (a) Total DoS for $x=0,0.5,1,2$, and 3; and (b) local DoS (LDoS) for CosSb22 (black), Te (red), and Ge (blue) in $\mathrm{CosSb}_{22} \mathrm{TeGe}$ (i.e., Co4Sb11 Te0.5Ge 0.5 with $x=0.5$ ). DoS results in (a) indicate that the bandgap decreases with increasing $x$. Part (b) shows an interaction between the local Te and Ge densities indicating orbital hybridization.

We further studied the local DoS (LDoS), which projected the density on each metal site. Through this approach, the density of states can be split into contributions from each individual atom. When these corresponding plots overlap, it suggests that each atom is contributing to the density of states at that given energy level, implying orbital hybridization. Figure $4.4 \mathrm{~b}$ illustrates the orbital hybridization through the energy bands of $-13 \mathrm{eV}$ to $5 \mathrm{eV}$ between $\mathrm{Co}$ and $\mathrm{Te} / \mathrm{Ge}$ substitution at $x=0.5$. It indicates the covalent characteristics of $\mathrm{Co}-\mathrm{Te} / \mathrm{Ge}$ bonds due to the overlap of orbital energies. As the $\mathrm{Te} / \mathrm{Ge}$ concentration increases, the average $\mathrm{Co}-\mathrm{Te} / \mathrm{Ge}$ bond distance decreases because of the stronger covalent nature of the bond.

Further analysis of band alignment facilitates the engineering of the skutterudite compounds for specific thermoelectric applications. The band structures of $\mathrm{Co}_{4} \mathrm{Sb}_{12}-$ ${ }_{2 \mathrm{x}} \mathrm{Te}_{\mathrm{x}} \mathrm{Ge}_{\mathrm{x}}$ for $x=0,0.5$, and 3 were calculated along lines connecting high symmetry points in the Brillouin zone as shown in Figure 4.5. The perfect $\operatorname{CoSb}_{3}(x=0)$ is a direct 
bandgap semiconductor with a calculated gap of $0.16 \mathrm{eV}$. The valence band maximum $(\mathrm{VBM})$ and conduction band minimum $(\mathrm{CBM})$ are both located at the $\Gamma$ point. There also exists an indirect gap at some lower-symmetry point having a local maximum between $\mathrm{M}$ and $\Gamma$ that is separated from the CBM by $0.50 \mathrm{eV}$. As Te/Ge substitutions are introduced $(x=0.5)$, the material remains a direct bandgap semiconductor but with a narrower size of $0.13 \mathrm{eV}$. This is caused by the decrease in the $\mathrm{CBM}$ at the $\Gamma$ point from $0.084 \mathrm{eV}$ to $0.066 \mathrm{eV}$. Also, the local maximum at the $\mathrm{M}$ point increases (from $-0.536 \mathrm{eV}$ to -0.355 $\mathrm{eV}$, giving rise to a shorter indirect gap of $0.42 \mathrm{eV}$ that now exists from $\mathrm{M} \rightarrow \Gamma$ highsymmetry points. This implies that with adjusting $x$ values, the compound could shift from a direct to an indirect bandgap semiconductor, creating more potential applications for the compounds. To confirm our prediction, we observed $x=3$ (Figure 4.5c) which shows the smaller bandgap of $0.11 \mathrm{eV}$, suggesting a high electrical conductivity. Both the VBM peak and CBM trough at the $\Gamma$ point split, forming an indirect gap. 

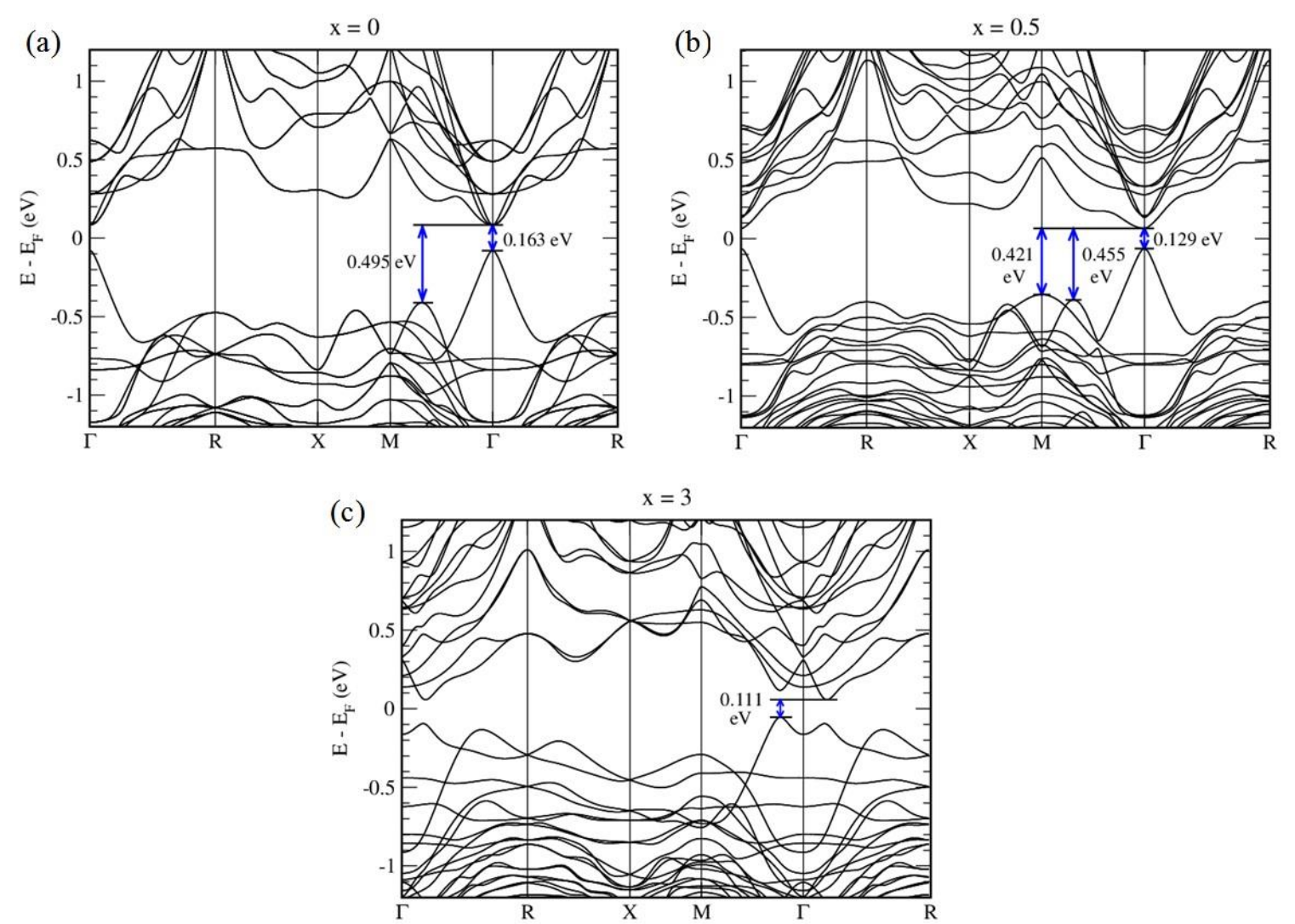

Figure 4.5. Electronic band structure diagrams of Co4Sb12-2xTexGe for (a) $x=0$, (b) $x=0.5$, and (c) $x=3$. Blue arrows indicate the changes in the direct and indirect bandgaps. As substitution increases, the bandgap decreases from (a) $0.16 \mathrm{eV}$ to (b) $0.13 \mathrm{eV}$, and eventually to (c) $0.11 \mathrm{eV}$ for the highest-doped structure investigated $(x$ $=3$ ). Also note the transition from direct to indirect bandgap semiconducting behavior in (c).

The electronic structure results indicate a strong Te/Ge substitution dependence in $\mathrm{Co}_{4} \mathrm{Sb}_{12-2 \mathrm{x}} \mathrm{Te}_{\mathrm{x}} \mathrm{Ge}_{\mathrm{x}}$, motivating us to investigate further increasing $x$ to the full substitution of $\mathrm{Sb}$ with $\mathrm{Te} / \mathrm{Ge}\left(x=6\right.$ or $\left.\mathrm{Co}_{4} \mathrm{Te}_{6} \mathrm{Ge}_{6}\right)$. Figure 4.6 shows electronic DoS for $x=6$ (full substitution) in comparison with that for $x=3$ (half substitution) and different atomic arrangements for $\mathrm{Sb}, \mathrm{Te}$, and Ge. In Figure 4.6b, the black line depicts the electronic DoS for $x=6$, where there is no bandgap and the local minimum in DoS at the Fermi level also has a higher value than that of $x=3$ (see Figure 4.6a). This suggests a generally increasing conductivity with increasing $x$. Interestingly, we found that atomic arrangements for $\mathrm{Sb}, \mathrm{Te}$, and $\mathrm{Ge}$ in the pnictogen rings affect the bandgap size. As seen 
in Figure 4.6c, configuration 1 involves the least amount of "order" where the Sb, Te, and Ge atoms are randomly arranged throughout the $\mathrm{Sb}$ sites. This is the configuration used for all substituted structures in Figure 4.4a. Ordering refers to having some pnictogen rings composed of entirely $\mathrm{Sb}$ and/or having some only $2 \mathrm{Te} / 2 \mathrm{Ge}$ rings arranged such that the Te and Ge atoms are at alternating sites around the ring. Configuration 2 is an example of this for the $x=3$ system. Finally, configuration 3 involves no randomly ordered rings, demonstrating that all $\mathrm{Sb}$ atoms are only sharing $\mathrm{Sb}$ rings at $x=3$ and all Te and Ge atoms are only sharing $2 \mathrm{Te} / 2 \mathrm{Ge}$ rings arranged as explained above. As a result, pnictogen ring "ordering” increases from configuration 1 to configuration 3 . We found that increasing the ring ordering opens up a bandgap and further increases the gap to as much as $0.32 \mathrm{eV}$ and $0.33 \mathrm{eV}$ for $x=3$ and $x=6$, respectively (see Figure 4.6a and Figure 4.6b). Our computational results emphasize the importance for understanding the atomic structure of these materials and the atomic structure-electrical property relationships. 

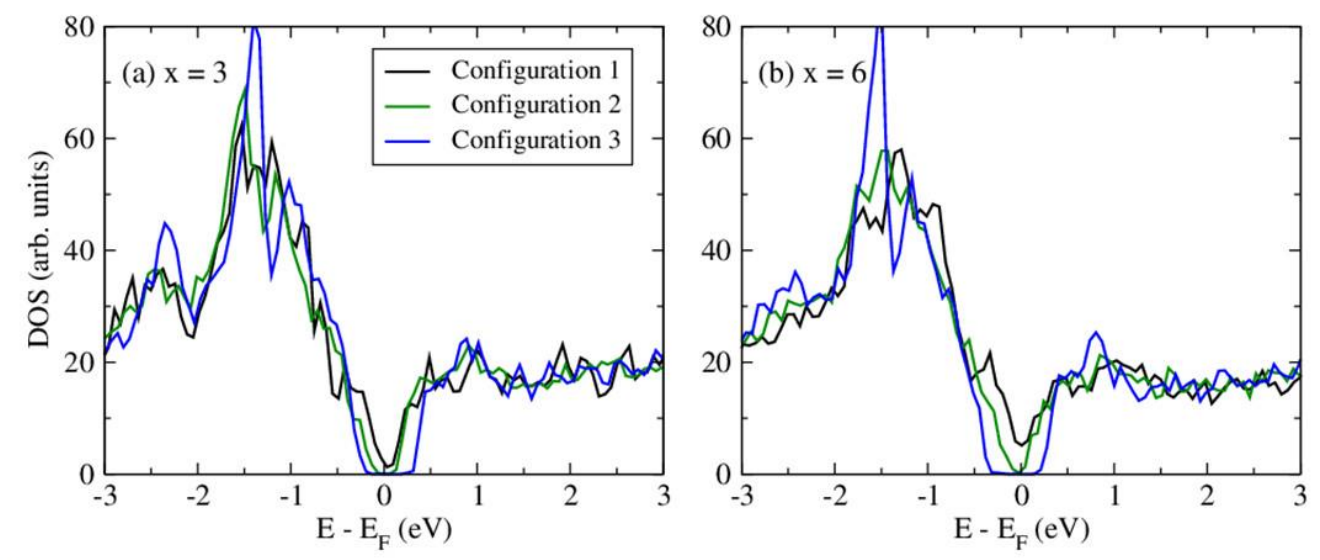

(c)

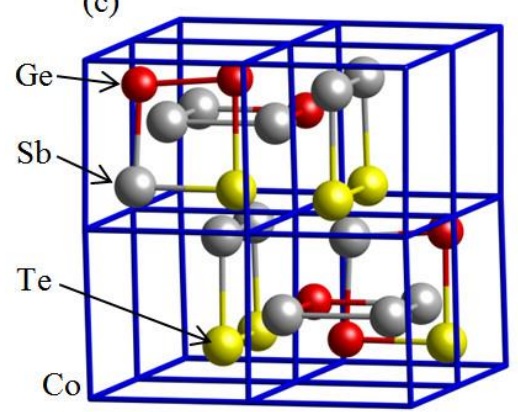

Configuration 1

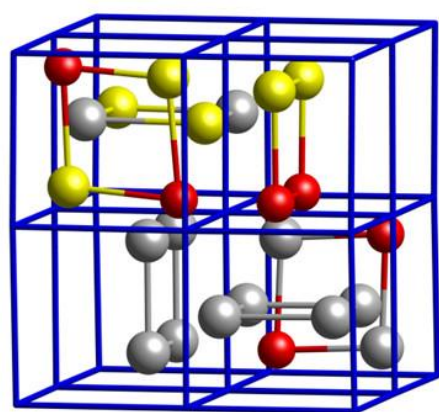

Configuration 2

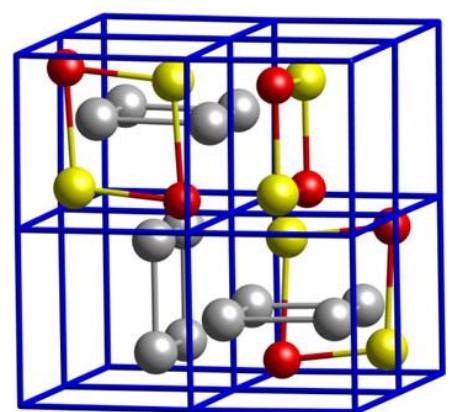

Configuration 3

Figure 4.6. Density of states plots for $\mathrm{Co4Sb}_{12-2 \mathrm{x}} \mathrm{Te}_{\mathrm{x}} \mathrm{Ge}_{\mathrm{x}}$ where (a) $x=3$ and (b) $x=$ 6. Fermi energy is zero. The black, green, and blue plots depict the randomly ordered (configuration 1), the partially ordered (configuration 2), and fully ordered (configuration 3) pnictogen ring configurations. The pnictogen ring ordering increasingly opens up the bandgap. An example of each pnictogen ring configuration (depicted using the $x=3$ structure) is shown in part (c) where the blue line shows CoCo bonds and the gray, yellow, and red spheres depict Sb, Te, and Ge atoms, respectively.

\section{$\underline{\text { Phonon Properties }}$}

The scattering of phonon modes in a material hinders thermal transport, which results in reduced lattice thermal conductivity $\kappa_{l}$. There are a variety of methods for increasing phonon scattering, including nanostructuring, alloying, introducing more disorder or interfaces, and changing the structure or composition. ${ }^{35}$ By analyzing the phonon dispersion relations of a material, one can estimate the effects of fillers or substitutions on the resulting phonon properties. For example, the phonon group velocity 
$v_{k \lambda}$ can be estimated by observing the slopes or gradients of the phonon dispersion data through the equation

$$
v_{k \lambda}=\frac{\partial \omega}{\partial k}
$$

where $\omega$ is the phonon frequency and $k$ is the wave vector. The phonon group velocity is then related to the lattice thermal conductivity through the following equation:

$$
\kappa_{l}=\frac{1}{3 V N_{k}} \sum_{k \lambda} c_{k \lambda} v_{k \lambda}^{2} \tau_{k \lambda}
$$

where $V$ is the volume of the unit cell, $N_{k}$ is the number of $k$-points, $c$ is the heat capacity, and $\tau$ is the phonon relaxation time for the given phonon branch, $\lambda \cdot{ }^{36,37}$ From this, the lattice thermal conductivity can be estimated qualitatively by analyzing the slope of the phonon dispersion results. Figure 4.7 shows phonon DoS data for the large 136-atom $\mathrm{Ca}_{4} \mathrm{Ce}_{4} \mathrm{Fe}_{32} \mathrm{Sb}_{96}$ compound compared to that of $\mathrm{Fe}_{8} \mathrm{Sb}_{24}$. Phonon dispersion data were also calculated but due to the large number of modes $(136 \times 3=408$ modes $)$ for the CaCefilled system, the results were more clearly displayed via the phonon DoS instead. We found that the incorporation of $\mathrm{Ca}$ and $\mathrm{Ce}$ filler atoms broadens the phonon bands, implying an increase in phonon scattering typically observed in filled skutterudite systems. The optical modes are also significantly flatter than the acoustic modes, suggesting that the lattice thermal conductivity for this material is dominated by the acoustic modes. 


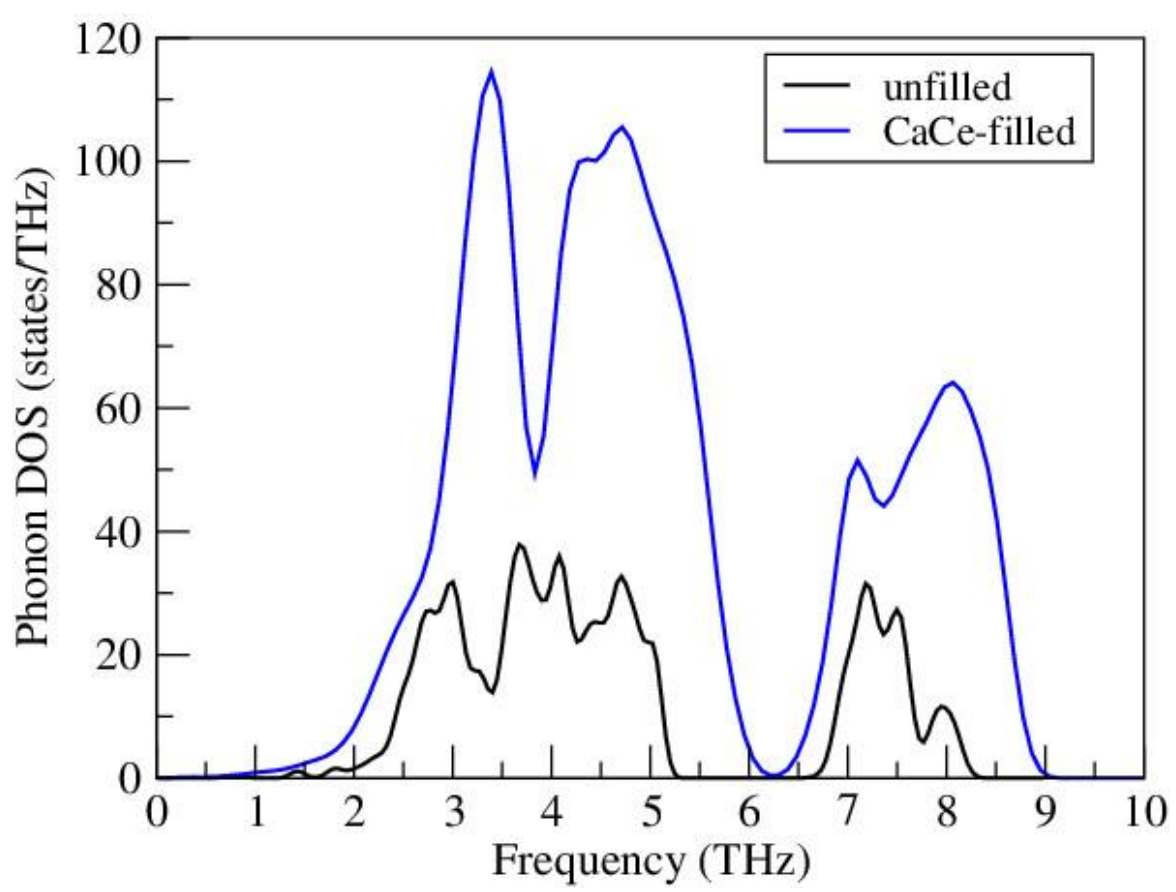

Figure 4.7. Phonon density of states for unfilled $\mathrm{Fe}_{8} \mathrm{Sb}_{24}$ (black) and CaCe-filled $\mathrm{Ca}_{4} \mathrm{Ce}_{4} \mathrm{Fe}_{32} \mathrm{Sb}_{96}$ (blue). To account for the proper $\mathrm{Ca} / \mathrm{Ce}$ filler atom ordering which provides the most stable structure (and most promising thermoelectric performance) of the $\mathrm{Ca}_{0.5} \mathrm{Ce}_{0.5} \mathrm{Fe}_{4} \mathrm{Sb}_{12}$ system, the larger 136-atom supercell was used for the "CaCefilled" structure.

Similarly, the effects of substitution on lattice thermal conductivity were also estimated by analyzing the phonon properties. Figure $4.8 \mathrm{a}$ shows the phonon dispersion relation for the $\mathrm{Co}_{4} \mathrm{Sb}_{12-2 \mathrm{x}} \mathrm{Te}_{\mathrm{x}} \mathrm{Ge}_{\mathrm{x}}$ compound at $x=0$. Figure $4.8 \mathrm{~b}$ compares the acoustic modes for the $\mathrm{Co}_{4} \mathrm{Sb}_{12-2 \mathrm{x}} \mathrm{Te}_{\mathrm{x}} \mathrm{Ge}_{\mathrm{x}}$ compounds at $x=0,3$, and 6 , where the $\mathrm{x}$-axes for each data set is normalized to the values of the compound at $x=6$ (for the purpose of comparison). The compound at $x=3$ has the lowest phonon dispersion gradient which could indicate that it has the lowest lattice thermal conductivity. Figure $4.8 \mathrm{c}$ compares the phonon density of states for the three compounds. It shows a broadening of the phonon bands, particularly for the higher-frequency band, for the compounds with Te and Ge substitutions at $x=3$ and 6 . Ge substitutions contribute to the higher frequency modes since Ge has a lower atomic weight than $\mathrm{Sb}$. In contrast, the modes at the lower 
frequencies are associated with the heavier Te substitutions. $x=0$ exhibits a sizable gap between the higher-frequency band and the lower band. As substitution increases, this gap decreases and is eventually eliminated at $x=6$. This spreading of optical modes indicates a reduction in lattice thermal conductivity. For either $x=3$ or $x=6$, the optical modes are significantly flatter than the acoustic modes, suggesting that the lattice thermal conduction is likely dominated by the acoustic modes in these compounds.

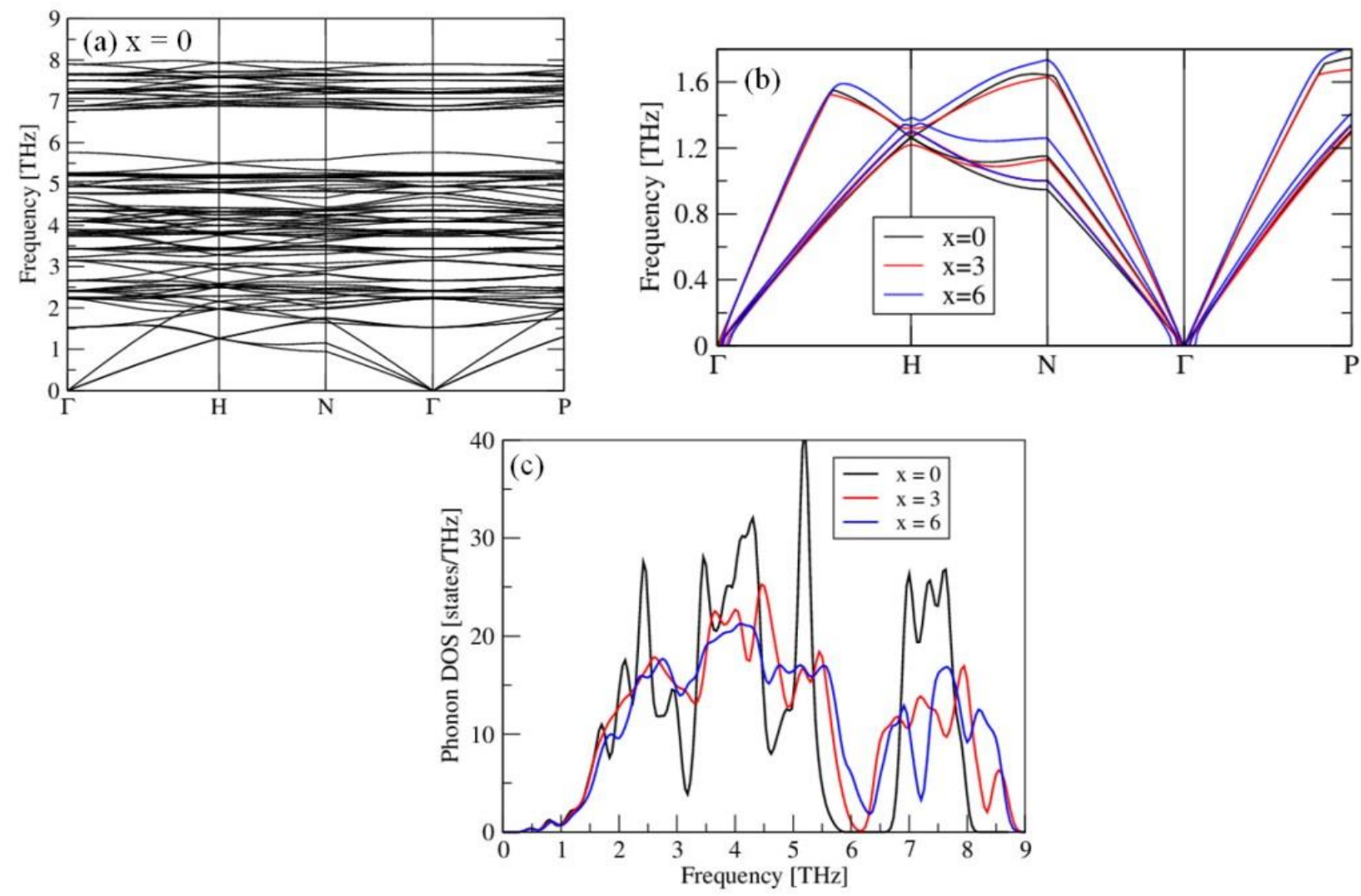

Figure 4.8. Phonon property calculations. (a) Phonon dispersion relation for $\mathrm{Co}_{4} \mathrm{Sb}_{12-2 \mathrm{x}} \mathrm{Te}_{\mathrm{x}} \mathrm{Ge}_{\mathrm{x}}$ at $x=0$. Similar plots were made for $x=3$ and $x=6$ but for brevity, only their acoustic modes (along with those of $x=0$ ) are given in (b), and (c) total phonon density of states for each compound, projected across the full range of mode frequencies.

\subsection{Conclusion}

We have investigated the crystal structures, electrical properties, and thermal properties of filled $\mathrm{Ca}_{0.5} \mathrm{Ce}_{0.5} \mathrm{Fe}_{4} \mathrm{Sb}_{12}$ and substituted $\mathrm{Co}_{4} \mathrm{Sb}_{12-2 \mathrm{x}} \mathrm{Te}_{\mathrm{x}} \mathrm{Ge}_{\mathrm{x}}$ compounds $(x=0$, 
$0.5,1,2,3$, and 6) using a DFT-based approach. The stable $\mathrm{Ca} / \mathrm{Ce}$ ordering required the use of a large $\mathrm{Ca}_{4} \mathrm{Ce}_{4} \mathrm{Fe}_{32} \mathrm{Sb}_{96}$ supercell with each filler atom occupying alternating (110) planes. For the $\mathrm{Co}_{4} \mathrm{Sb}_{12-2 \mathrm{x}} \mathrm{Te}_{\mathrm{x}} \mathrm{Ge}_{\mathrm{x}}$ compounds, structural distortion occurs with $\mathrm{Te} / \mathrm{Ge}$ substitution. At $x=3$ (i.e., 1:1 Sb:Te/Ge ratio), four-member pnictogen square rings have the largest distortion and become parallelograms with an angle of $81^{\circ}$. Electronic structure calculations indicate that the $\mathrm{Ca} / \mathrm{Ce}$ double-filled $\mathrm{FeSb}_{3}$ is conductive with no bandgap. The bandgap of $\mathrm{Co}_{4} \mathrm{Sb}_{12-2 \mathrm{x}} \mathrm{Te}_{\mathrm{x}} \mathrm{Ge}_{\mathrm{x}}$ varies with Te/Ge substitution concentration leading to a reduction in bandgap and therefore an increase of electrical conductivity. The covalent characteristics of $\mathrm{Co}-\mathrm{Te} / \mathrm{Ge}$ bonds account for orbital hybridizations between Co and Te/Ge. Band structures exhibit the change of band alignments near the Fermi energy as $x$ increases. At $x=3$, the compound transfers from a direct to indirect band-gap semiconductor. We also found a strong correlation between the ordering/configuration of pnictogen rings and the electronic bandgap for these compounds. We predicted the thermal conductivity change through the analysis of phonon DoS and phonon dispersion relations for the compounds. The phonon bands are broadened with the incorporation of either fillers or substitutions. Lattice thermal conductivity change is controlled by the acoustic phonon modes in both cases. For the $\mathrm{Co}_{4} \mathrm{Sb}_{12-2 \mathrm{x}} \mathrm{Te}_{\mathrm{x}} \mathrm{Ge}_{\mathrm{x}}$ compounds, phonon scattering and acoustic mode gradient change are evident as substitution increases. The compound at $x=3$ is expected to have the lowest lattice thermal conductivity. Our work predicts improved thermoelectric properties via filler and substitutional doping. More work will be conducted to confirm the predictions through further $Z T$ calculations and measurements. 
The overall approach for analyzing these skutterudite systems and investigating their filler and substitution effects can be used to facilitate research on 2D TMD systems in the following chapters. Similar DFT calculations were performed to relax and analyze the structure of 2D TMD materials. Both the methodology for calculating electron and phonon dispersion spectra and the analysis of electrical and phonon properties were applied to our 2D TMD research. The supercell approach for investigating dopants was also used to build metal-site substituted $\mathrm{MX}_{2}$ structures for Chapter Six and lateral heterostructures for Chapter Seven.

\section{Acknowledgements}

This work was partially supported by the NSF EAPSI Fellowship Grant No. 1414593. Computing facilities were provided by Boise State University's R1 cluster, and Idaho National Laboratory's high performance computing (HPC) center. The authors thank Dr. Jin Zhao at the University of Science and Technology of China for helpful discussions. 


\section{References}

(1) Bjerg, L.; Madsen, G. K. H.; Iversen, B. B. Enhanced Thermoelectric Properties in Zinc Antimonides. Chem Mater 2011, 23, 3907.

(2) Tan, G. J.; Wang, S. Y.; Li, H.; Yan, Y. G.; Tang, X. F. Enhanced thermoelectric performance in zinc substituted p-type filled skutterudites $\mathrm{CeFe}_{4}$ ${ }_{x} \mathrm{Zn}_{\mathrm{x}} \mathrm{Sb}_{12}$. Journal of Solid State Chemistry 2012, 187, 316.

(3) Chrissafis, K.; Bikiaris, D. Can nanoparticles really enhance thermal stability of polymers? Part I: An overview on thermal decomposition of addition polymers. Thermochimica Acta 2011, 523, 1.

(4) Dragoman, D.; Dragoman, M. Giant thermoelectric effect in graphene. Appl Phys Lett 2007, 91, 203116.

(5) Dyck, J. S.; Chen, W. D.; Uher, C.; Chen, L.; Tang, X. F.; Hirai, T. Thermoelectric properties of the n-type filled skutterudite Ba0.3Co4Sb12 doped with Ni. J Appl Phys 2002, 91, 3698.

(6) Freeman, D. D.; Choi, K.; Yu, C. N-Type Thermoelectric Performance of Functionalized Carbon Nanotube-Filled Polymer Composites. Plos One 2012, 7, e47822.

(7) Zhao, Y.; Tang, G. S.; Yu, Z. Z.; Qi, J. S. The effect of graphite oxide on the thermoelectric properties of polyaniline. Carbon 2012, 50, 3064.

(8) Lan, Y.; Minnich, A. J.; Chen, G.; Ren, Z. Enhancement of Thermoelectric Figure-of-Merit by a Bulk Nanostructuring Approach. Advanced Functional Materials 2010, 20, 357.

(9) Wong-Ng, W.; Kaduk, J. A.; Tan, G.; Yan, Y.; Tang, X. X-ray powder reference patterns of the $\mathrm{Fe}(\mathrm{Sb} 2+\mathrm{xTe} 1-\mathrm{x})$ skutterudites for thermoelectric applications. Powder Diffraction 2014, 29, 260.

(10) Tan, G. J.; Liu, W.; Chi, H.; Su, X. L.; Wang, S. Y.; Yan, Y. G.; Tang, X. F.; Wong-Ng, W.; Uher, C. Realization of high thermoelectric performance in p- 
type unfilled ternary skutterudites FeSb2+xTe1-x via band structure modification and significant point defect scattering. Acta Materialia 2013, 61, 7693.

(11) Yan, Y. G.; Wong-Ng, W.; Kaduk, J. A.; Tan, G. J.; Xie, W. J.; Tang, X.

F. Correlation of thermoelectric and microstructural properties of $\mathrm{p}$-type CeFe4Sb12 melt-spun ribbons using a rapid screening method. Appl Phys Lett 2011, 98 .

(12) He, T.; Chen, J. Z.; Rosenfeld, H. D.; Subramanian, M. A. Thermoelectric properties of indium-filled skutterudites. Chem Mater 2006, 18, 759.

(13) Huang, B.; Kaviany, M. Filler-reduced phonon conductivity of thermoelectric skutterudites: Ab initio calculations and molecular dynamics simulations. Acta Materialia 2010, 58, 4516.

(14) Hara, R.; Inoue, S.; Kaibe, H. T.; Sano, S. Aging effects of large-size ntype CoSb3 prepared by spark plasma sintering. Journal of Alloys and Compounds 2003, 349, 297.

(15) Kim, H.; Kaviany, M.; Thomas, J. C.; Van der Ven, A.; Uher, C.; Huang, B. L. Structural Order-Disorder Transitions and Phonon Conductivity of Partially Filled Skutterudites. Phys Rev Lett 2010, 105, 265901.

(16) Luo, P. F.; Tang, X. F.; Xiong, C.; Zhang, Q. J. Effect of multiwalled carbon nanotubes on the thermoelectric properties of p-type $\mathrm{Ba} 0.3 \mathrm{FeCo} 3 \mathrm{Sb} 12$ compounds. Acta Phys Sin-Ch Ed 2005, 54, 2403.

(17) Morelli, D. T.; Caillat, T.; Fleurial, J. P.; Borshchevsky, A.; Vandersande, J.; Chen, B.; Uher, C. Low-Temperature Transport-Properties of P-Type Cosb3. Phys Rev B 1995, 51, 9622.

(18) Caillat, T.; Borshchevsky, A.; Fleurial, J. P. Properties of single crystalline semiconducting CoSb3. J Appl Phys 1996, 80, 4442.

(19) Yang, J.; Zhang, W.; Bai, S. Q.; Mei, Z.; Chen, L. D. Dual-frequency resonant phonon scattering in BaxRyCo4Sb12 (R=La, Ce, and Sr). Appl Phys Lett 2007, 90, 192111. 
(20) Yang, J.; Endres, M. G.; Meisner, G. P. Valence of Cr in skutterudites: Electrical transport and magnetic properties of Cr-doped CoSb3. Phys Rev B 2002, 66 .

(21) Yang, J.; Morelli, D. T.; Meisner, G. P.; Chen, W.; Dyck, J. S.; Uher, C. Influence of electron-phonon interaction on the lattice thermal conductivity of Co1-xNixSb3. Phys Rev B 2002, 65.

(22) Yan, Y. G.; Wong-Ng, W.; Li, L.; Levin, I.; Kaduk, J. A.; Suchomel, M. R.; Sun, X.; Tan, G. J.; Tang, X. F. Structures and thermoelectric properties of double-filled (CaxCe1-x)Fe4Sb12 skutterudites. Journal of Solid State Chemistry 2014, 218.

(23) Kresse, G.; Furthmuller, J. Efficient iterative schemes for ab initio totalenergy calculations using a plane-wave basis set. Phys Rev B 1996, 54, 11169.

(24) Perdew, J. P.; Burke, K.; Ernzerhof, M. Generalized gradient approximation made simple. Phys Rev Lett 1996, 77, 3865.

(25) Blöchl, P. E. Projector augmented-wave method. Phys Rev B 1994, 50, 17953.

(26) Kresse, G.; Joubert, D. From ultrasoft pseudopotentials to the projector augmented-wave method. Phys Rev B 1999, 59, 1758.

(27) Liechtenstein, A. I.; Anisimov, V. I.; Zaanen, J. Density-functional theory and strong interactions: Orbital ordering in Mott-Hubbard insulators. Phys Rev B 1995, 52, R5467.

(28) Parlinski, K.; Li, Z. Q.; Kawazoe, Y. First-principles determination of the soft mode in cubic ZrO2. Phys Rev Lett 1997, 78, 4063.

(29) Togo, A.; Oba, F.; Tanaka, I. First-principles calculations of the ferroelastic transition between rutile-type and $\mathrm{CaCl}(2)$-type $\mathrm{SiO}(2)$ at high pressures. Phys Rev B 2008, 78, 134106.

(30) Su, X.; Yan, Y.; Wong-Ng, W.; Williamson, I.; Her, L. J.-Y.; Kaduk, J. A.; Li, L. Powder Diffraction 2015, in preparation. 
(31) Rietveld, H. M. A Profile Refinement Method for Nuclear and Magnetic Structures. J Appl Crystallogr 1969, 2, 65.

(32) Pyykko, P.; Atsumi, M. Molecular Single-Bond Covalent Radii for Elements 1-118. Chemistry: A European Journal 2009, 15, 186.

(33) Su, X. L.; Li, H.; Guo, Q. S.; Tang, X. F.; Zhang, Q. J.; Uher, C. Structure and Thermoelectric Properties of Te- and Ge-Doped Skutterudites CoSb2.875xGe0.125Tex. Journal of Electronic Materials 2011, 40, 1286.

(34) Su, X. L.; Li, H.; Wang, G. Y.; Chi, H.; Zhou, X. Y.; Tang, X. F.; Zhang, Q. J.; Uher, C. Structure and Transport Properties of Double-Doped CoSb2.75Ge0.25-xTex (x=0.125-0.20) with in Situ Nanostructure. Chem Mater 2011, 23, 2948.

(35) Snyder, G. J.; Toberer, E. S. Complex thermoelectric materials. Nature Materials 2008, 7, 105.

(36) Luo, T. F.; Garg, J.; Shiomi, J.; Esfarjani, K.; Chen, G. Gallium arsenide thermal conductivity and optical phonon relaxation times from first-principles calculations. Europhysics Letters 2013, 101, 16001.

(37) Chung, J. D.; McGaughey, A. J. H.; Kaviany, M. Role of phonon dispersion in lattice thermal conductivity modeling. Journal of Heat Transfer: Transactions of the ASME 2004, 126, 376. 


\section{CHAPTER FIVE: HIGH-THROUGHPUT COMPUTATIONAL SCREENING OF ELECTRICAL AND PHONON PROPERTIES OF TWO-DIMENSIONAL TRANSITION METAL DICHALCOGENIDES*}

\footnotetext{
*This chapter includes modifications from the originally published version and is adapted from:
}

I. Williamson, A. Correa Hernandez, W. Wong-Ng, L. Li, "High-throughput computational screening of electrical and phonon properties of two-dimensional transition metal dichalcogenides.” JOM, 68(10) 2666 (2016). doi:10.1007/s11837-0162068-X.

Reproduced with permission of The Minerals, Metals, \& Materials Society 
High-throughput computational screening of electrical and phonon properties of two-dimensional transition metal dichalcogenides

\author{
Izaak Williamson $^{\mathrm{a}}$ \\ Andres Correa Hernandez ${ }^{\mathrm{a}}$ \\ Winnie Wong- $\mathrm{Ng}^{\mathrm{b}}$ \\ Lan $\mathrm{Li}^{\mathrm{a}, \mathrm{c}}$ \\ Published in: \\ JOM \\ October 2016 \\ ${ }^{a}$ Micron School of Materials Science and Engineering, Boise State University, \\ Boise, ID 83725. \\ ${ }^{b}$ Materials Measurement Science Division, National Institute of Standards and \\ Technology, \\ Gaithersburg, MD 20899. \\ ${ }^{c}$ Center for Advanced Energy Studies, \\ Idaho Falls, ID 83401.
}




\begin{abstract}
Two-dimensional transition metal dichalcogenides (2D TMDs) are of broadening research interest due to their novel physical, electrical, and thermoelectric properties. Having the chemical formula $\mathrm{MX}_{2}$, where $\mathrm{M}$ is a transition metal and $\mathrm{X}$ is a chalcogen, such materials present many possible combinations to consider for materials-by-design exploration. By identifying novel compositions and utilizing the lower dimensionality, which allows for improved thermoelectric performance (e.g., increased Seebeck coefficients without sacrificing electron concentration), $\mathrm{MX}_{2}$ materials are potential candidates for electronic and energy applications; however, to develop these materials for wide-scale use, it is crucial to comprehensively understand the compositional affects. This work investigates the structure, electronic, and phonon properties of 18 different $\mathrm{MX}_{2}$ material compositions as a benchmark to explore the impact of various elements. There is significant correlation between properties of constituent transition metals (atomic mass and radius) and the structure/properties of the corresponding 2D TMDs. As the mass of $\mathrm{M}$ increases, the $n$-type power factor and phonon frequency gap increases. Similarly, increases in the radius of M lead to increased layer thickness and Seebeck coefficient, $S$. Our results identify key factors to optimize $\mathrm{MX}_{2}$ compositions for desired performance.
\end{abstract}

\title{
5.1 Introduction
}

TMDs, having the chemical formula $\mathrm{MX}_{2}(\mathrm{M}=$ transition metal, $\mathrm{X}=$ chalcogen, e.g., $\mathrm{S}, \mathrm{Se}, \mathrm{Te}$ ), have received attention over the years for having interesting magnetic ${ }^{1,2}$ and photoelectric ${ }^{3}$ properties. Bulk three-dimensional transition metal dichalcogenides (3D TMDs) can exist as layered structures wherein each layer consists of a transition 
metal (M) sheet sandwiched between two chalcogen (X) sheets. These layers exist in one of two structure types: $2 \mathrm{H}$-type having $D_{6 h}$ point group symmetry and 1T-type having $D_{3 d}$ point-group symmetry. Strong covalent bonding exists within the $\mathrm{MX}_{2}$ layers (along the $a$ and $b$ axes) while much weaker van der Waals (vdW) interactions exist between the layers (along the $c$ axis). This allows individual $\mathrm{MX}_{2}$ layers to be separated via mechanical exfoliation. ${ }^{4}$ The separated 2D TMD materials have recently attracted much interest for a variety of applications including electronics, ${ }^{5-9}$ optoelectronics, ${ }^{10-13}$ catalysis, ${ }^{14,15}$ and thermoelectrics. ${ }^{16-19}$ Thermoelectrics convert waste heat into electric power, governed by figure of merit, $Z T$ (see Equation 2.1). Common thermoelectric materials include clathrates, ${ }^{20}$ half-Heusler alloys, ${ }^{21}$ and skutterudites.${ }^{22-25}$ Reduced dimensionality offers the potential for smaller device features and has been shown to greatly reduce short-channel effects, increase the on-off ratio, and reduce switching voltage in field-effect transistors (FET). ${ }^{7,10}$ In addition, the quantum confinement effect and sharp features in the electronic DoS are beneficial for greatly improving thermoelectric properties, such as increasing $S$ in 2D TMDs compared to that of the bulk structures. ${ }^{26-28}$ It is also predicted that the lower dimensionality increases diffusive boundary scattering, effectively reducing the thermal conductivity, $\kappa$. Lowering the dimensionality of $\mathrm{Si}$, for example, has shown strong reductions in thermal conductivity and implications for enhanced thermoelectric performance. ${ }^{29,30}$ Each of these factors contributes to the strong motivation for further understanding the behavior of 2D TMDs.

With a large number of potential 2D TMD compounds, it is important to identify key structure-property-performance relationships in order to screen preferred materials. A few studies have been performed to analyze the stability and electronic structure of 2D 
TMDs and TMOs (transition metal dioxides) using DFT. ${ }^{5,6}$ These offer a general analysis of trends across a broad range of compositions and identify most structures to be semiconducting with small indirect bandgaps. Rasmussen et al. ${ }^{6}$ found that only $\mathrm{CrX}_{2}$, $\mathrm{MoX}_{2}$, and $\mathrm{WX}_{2}$ systems exhibit direct bandgaps. From these works, it is useful to identify more favorable 2D TMD compositions in terms of stability, electrical properties, and optical properties; however, none have been directed at what compositions are possible. Graedel et al. ${ }^{31}$ developed a method for quantifying the environmental implications, supply risk, and vulnerability to supply restrictions for 62 transition metals and metalloids. These "criticality scores" can be used to identify preferred materials and narrow the focus for screening potential compositions in order to develop highperformance, cost-effective, and environmentally friendly materials.

In this work, we used existing stability reference data in conjunction with criticality scores to identify transition metals and resulting 2D TMD compounds to represent a narrowed scope of feasible options for $\mathrm{MX}_{2}$ materials screening purposes. $\mathrm{A}$ list of six transition metals was selected to offer a range of common oxidation states, atomic masses, and atomic radii. Combined with three chalcogens $-\mathrm{S}, \mathrm{Se}$, and $\mathrm{Te}-$ this amounted to 18 total compounds. The geometries, electrical properties, and phonon properties of these compounds were investigated through first-principles calculation and analyzed with respect to physical properties of the transition metals. This unique approach offers a means of identifying general trends in constituent elements in order to predict structure-properties-performance relationships in resulting 2D TMDs. 


\subsection{Computational Methods}

Structural and electronic structure calculations were conducted using the PAW method $^{32,33}$ within the framework of DFT via the VASP code. ${ }^{34}$ The PBE ${ }^{35}$ formalism was employed within the spin-dependent GGA. A plane-wave expansion cutoff of 500 $\mathrm{eV}$ was used along with an automatic $\Gamma$-centered $12 \times 12 \times 1 k$-point mesh Brillouin zone integration for the initial geometry optimizations. A Gaussian smearing of $0.025 \mathrm{eV}$ was used to account for Fermi surface broadening while atomic positions and basis vectors were relaxed to within $0.01 \mathrm{eV} / \AA$. To avoid image interaction, $\mathrm{MX}_{2}$ sheets were separated by a distance $>20 \AA$. After geometry optimizations, the electronic structure calculations were performed with an increased $k$-point mesh of $24 \times 24 \times 1$ while strong correlation effects were accounted for using the DFT+U scheme for all transition metals. The on-site Coulomb potentials of $\mathrm{U}=4.38,8.0,3.0,8.0,6.0$, and $8.0 \mathrm{eV}$ were used for Mo, Ni, Sc, Ti, V, and $\mathrm{W}$, respectively. ${ }^{36}$ The on-site exchange potential, $\mathrm{J}=1.0 \mathrm{eV}$, was used for all metals.

Force constants used to estimate phonon properties were calculated using DFPT following the method of Parlinski-Li-Kawazoe. ${ }^{37}$ To calculate the real-space force constants, long-range effects were accounted for by using a converged supercell size of $3 \times 3 \times 2$ and a corresponding $k$-point mesh of $4 \times 4 \times 2$. The resulting force constants were used to generate phonon DoS and partial phonon DoS. ${ }^{38}$

In bulk TMD structures, it is necessary to employ vdW corrections to account for the inter-sheet interactions as they are determined to be largely controlled by vdW forces. Indeed, our own comparison of bulk $\mathrm{MoS}_{2}$ (using the Grimme DFT-D2 method ${ }^{39}$ and a $12 \times 12 \times 4 k$-point mesh) with that of reported experimental values ${ }^{40}$ revealed that 
accounting for vdW forces increases the accuracy by about $24.5 \%$ and $11.9 \%$ for sheet separation distance and cell volume, respectively; however, since the bonding within the 2D TMD sheets is primarily strongly covalent, it may not be necessary to include such corrections for calculations involving the isolated sheets. We tested for $2 \mathrm{D} \mathrm{MoS}_{2}$ and found that incorporating vdW corrections gave the same lattice constant and layer thickness values (to within $0.3 \%$ error) yet produced an indirect bandgap of $1.65 \mathrm{eV}$. Since $2 \mathrm{D} \mathrm{MoS} 2$ is known to be a direct bandgap semiconductor, ${ }^{41}$ it would seem that DFT-D2-based vdW corrections actually have a negative impact on 2D TMD systems. Due to the minimal difference in geometry and potential error in the electronic structure calculations, we did not employ vdW in this work.

Similar to the skutterudite modeling approach, each of the DFT parameters in this chapter were optimized through convergence tests. These tests are meant to provide the most accurate ground state that can be reasonably obtained while investigating eighteen different compounds. Our calculated lattice constants, bond lengths and angles, and electronic bandgaps were compared with literature values where available ${ }^{5,6}$ to validate our results.

\subsection{Results}

\section{$\underline{\text { Structure Screening }}$}

The unique physical, electrical, and thermal properties of 2D TMDs are of great research interest for a wide range of applications. However, there are many possible $\mathrm{MX}_{2}$ combinations to consider. Work by Graedel et al. highlights the "criticality scores" for transition metals, allowing for the comparison of three important factors to consider for materials design: environmental implications, supply risk, and vulnerability to supply 
restrictions. ${ }^{31}$ By comparing this list with the stability results from Ataca et al. ${ }^{5}$ and Rasmussen et al. ${ }^{6}$ we have narrowed the list of favorable transition metals to six: Mo, Ni, $\mathrm{Sc}, \mathrm{Ti}, \mathrm{V}$, and $\mathrm{W}$. These metals offer a broad range of atomic masses $(44.96-183.85 \mathrm{u}){ }^{42}$ atomic radii $(0.62-0.885 \AA),{ }^{43}$ and most common oxidation state $(+2$ to +6$)$. The comparison of the calculated properties with regard to these factors offers an effective approach towards identifying key relationships between constituent elements and desired structural, electrical, and thermal properties.

Table 5.1 Oxidation state, atomic mass (u), and atomic radius $(\AA)$ for each transition metal. The 2D TMDs $\left(\mathrm{MX}_{2}\right)$, consisting of a transition metal $(M)$ and chalcogen $(X)$, have either $2 \mathrm{H}$ - or 1T-type structures. Lattice parameter $a(\AA)$, layer thickness $t(\AA)$, and electronic bandgap $E_{g}(\mathrm{eV})$ of the corresponding TMD are also given. ${ }^{a}$ Reference. ${ }^{41}{ }^{b}$ Reference. ${ }^{42}$ Values taken from list of crystal radii with +4 charge and six-fold coordination. ${ }^{\mathrm{c}}$ Value for +3 charge. ${ }^{\mathrm{d}} \boldsymbol{E}_{F}$ sits on mid-gap state.

\begin{tabular}{|c|c|c|c|c|c|c|c|c|}
\hline$M$ & $\begin{array}{c}\text { Oxidation } \\
\text { state }\end{array}$ & $\begin{array}{c}\text { Atomic } \\
\text { mass }^{\mathrm{a}}(\mathrm{u})\end{array}$ & $\begin{array}{c}\text { Atomic } \\
\text { radius }^{\mathrm{b}}(\AA)\end{array}$ & $X$ & Type & $a(\AA)$ & $t(\AA)$ & $E_{g}(\mathrm{eV})$ \\
\hline $\mathrm{Mo}$ & +6 & 95.94 & 0.79 & $\mathrm{~S}$ & $2 \mathrm{H}$ & 3.182 & 3.128 & 1.69 \\
\hline & & & & $\mathrm{Se}$ & $2 \mathrm{H}$ & 3.319 & 3.338 & 1.46 \\
\hline & & & & $\mathrm{Te}$ & $2 \mathrm{H}$ & 3.549 & 3.611 & 1.10 \\
\hline $\mathrm{Ni}$ & +2 & 58.69 & 0.62 & $\mathrm{~S}$ & $1 \mathrm{~T}$ & 3.351 & 2.336 & 0.01 \\
\hline & & & & $\mathrm{Se}$ & $1 \mathrm{~T}$ & 3.545 & 2.479 & - \\
\hline & & & & $\mathrm{Te}$ & $2 \mathrm{H}$ & 3.720 & 2.927 & - \\
\hline $\mathrm{Sc}$ & +3 & 44.956 & $0.885^{\mathrm{c}}$ & $\mathrm{S}$ & $2 \mathrm{H}$ & 3.777 & 2.708 & $1.72^{\mathrm{d}}$ \\
\hline & & & & $\mathrm{Se}$ & $2 \mathrm{H}$ & 3.512 & 3.635 & - \\
\hline & & & & $\mathrm{Te}$ & $2 \mathrm{H}$ & 3.728 & 4.009 & - \\
\hline $\mathrm{Ti}$ & +4 & 47.88 & 0.745 & $\mathrm{~S}$ & $1 \mathrm{~T}$ & 3.398 & 2.854 & 1.18 \\
\hline & & & & $\mathrm{Se}$ & $2 \mathrm{H}$ & 3.485 & 3.239 & 0.92 \\
\hline & & & & $\mathrm{Te}$ & $2 \mathrm{H}$ & 3.736 & 3.571 & 0.30 \\
\hline $\mathrm{V}$ & +5 & 50.942 & 0.72 & $\mathrm{~S}$ & $1 \mathrm{~T}$ & 3.174 & 2.938 & - \\
\hline & & & & $\mathrm{Se}$ & $2 \mathrm{H}$ & 3.335 & 3.197 & 0.72 \\
\hline & & & & $\mathrm{Te}$ & $2 \mathrm{H}$ & 3.596 & 3.502 & 0.02 \\
\hline $\mathrm{W}$ & +6 & 183.85 & 0.80 & $\mathrm{~S}$ & $2 \mathrm{H}$ & 3.181 & 3.139 & 1.85 \\
\hline & & & & $\mathrm{Se}$ & $2 \mathrm{H}$ & 3.317 & 3.355 & 1.58 \\
\hline & & & & $\mathrm{Te}$ & $2 \mathrm{H}$ & 3.552 & 3.623 & 1.07 \\
\hline
\end{tabular}


The complete list of eighteen 2D TMDs used in this study is given in Table 5.1. The most common oxidation state, atomic mass, and atomic radius are listed for each transition metal (M). The resultant TMD forms either a $2 \mathrm{H}$ - or 1T-type phase. The TMD lattice parameter, $a$, is typically similar to the layer thicknesses except in the case of $\mathrm{ScS}_{2}$ and the $\mathrm{NiX}_{2}$ system (with layer thicknesses about $1 \AA$ smaller than $a$ ). Also, both the lattice parameter and layer thickness increase with the atomic number of $\mathrm{X}$. The range of values for both the lattice parameter and layer thickness noticeably decreases with increasing oxidation state of the transition metal. Similarly, this range also decreases with increasing atomic mass of the transition metal down to within $<0.5 \AA$ for both the $\mathrm{MoX}_{2}$ and $\mathrm{WX}_{2}$ systems $\left(\mathrm{m}_{\mathrm{Mo}}=95.94 \mathrm{u}, \mathrm{mw}_{\mathrm{w}}=183.85 \mathrm{u}\right)$. Figure 5.1 shows how the lattice parameter and layer thickness of the resulting 2D TMD are influenced by the atomic mass and atomic radius of the constituent transition metal. Interestingly, the layer thickness increases with atomic radius of the transition metal while the lattice parameter remains constant. The results reveal some materials design principles that lattice mismatch - causing a large instability in the formation of heterostructures - is minimal or negligible, even though 2D TMDs have large layer thickness differences. 

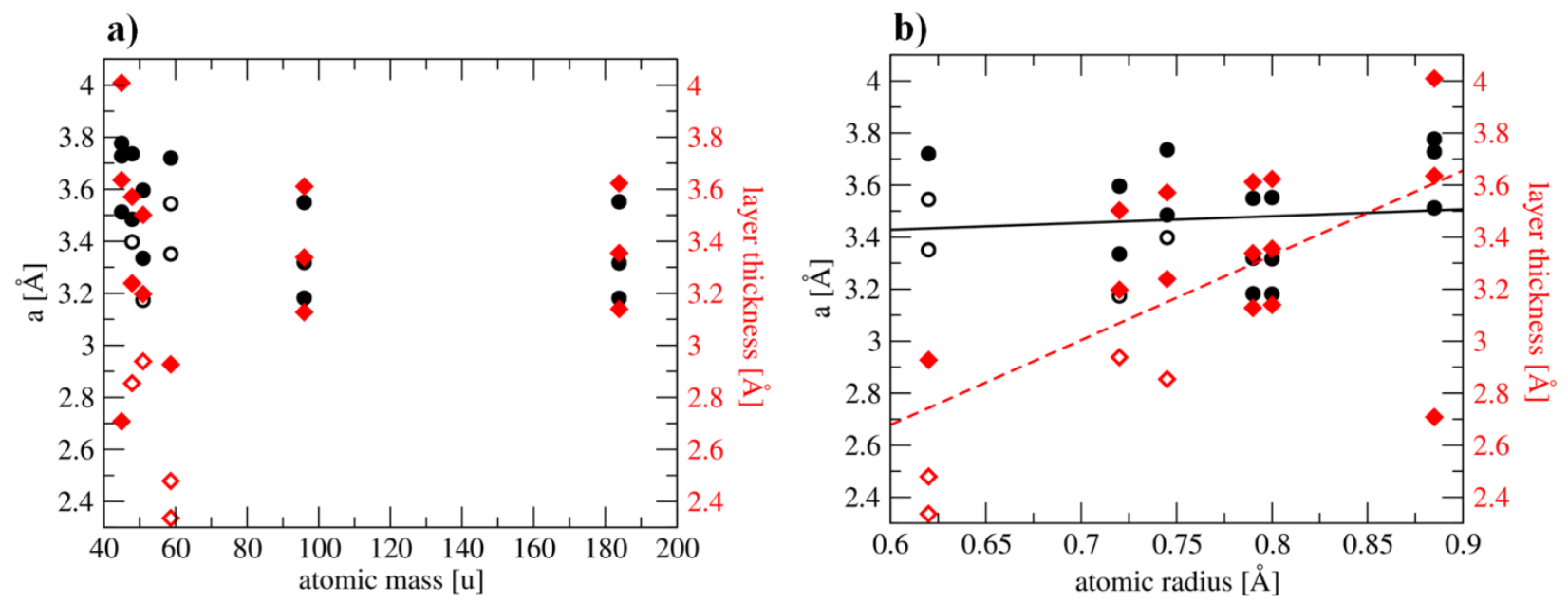

Figure 5.1. Effects of (a) atomic mass and (b) atomic radius of the transition metal on the lattice constant, $a$, and layer thickness of the resulting 2D TMD structure. Circles and diamonds refer to lattice constant and layer thickness values, respectively. Open and filled data points refer to 1T- and 2H-type $\mathbf{M X}_{2}$ structures, respectively. Solid and dashed linear trend lines have been added to plot (b) to guide the eye for lattice constant and layer thickness data, respectively.

\section{Electrical Property Screening}

Electronic structure calculations were performed for the 2D TMDs and determined that many of the systems exhibit semiconducting behavior. The $\mathrm{MoX}_{2}, \mathrm{TiX}_{2}$, and $\mathrm{WX}_{2}$ systems all have nonzero bandgaps with Fermi levels just above their respective VBM. The magnitude for each bandgap decreases with the increasing atomic number of $\mathrm{X}$. Specifically, the $\mathrm{MoX}_{2}$ and $\mathrm{WX}_{2}$ systems have bandgaps similar to each other, and they are the only systems with direct bandgaps. These results are consistent with the findings of Rasmussen et al. ${ }^{6}$ In contrast, the $\mathrm{NiX}_{2}, \mathrm{ScX}_{2}$, and $\mathrm{VX}_{2}$ systems all exhibit $p$ type semiconducting behavior with Fermi levels below the VBM. The only member of the $\mathrm{VX}_{2}$ system with a mid-gap Fermi level is $\mathrm{VSe}_{2}-$ a semiconductor with an indirect bandgap of $0.72 \mathrm{eV}$. Generally speaking, the compounds exhibiting the largest bandgaps are the ones that involve mid-level transition metal radii. The most conductive 
compounds have either very large $(\mathrm{Sc})$ or very small $(\mathrm{Ni})$ atomic radii, implying a Gaussian distribution of bandgaps across the range of atomic radii.

To further investigate electrical properties, the power factor $S^{2} \sigma$ (a crucial metric for the performance of thermoelectric materials, see Equation 2.1) and $S$ were also calculated for each TMD. The BTE was solved in order to calculate semi-classic transport coefficients. ${ }^{44}$ The $S$ data is shown in Figure 5.2 as a function of carrier concentration. Each calculation was evaluated for the temperature of $300 \mathrm{~K}$. Consistent with the bandgaps, the $\mathrm{MoX}_{2}, \mathrm{TiX}_{2}$, and $\mathrm{WX}_{2}$ systems show the largest-magnitude $S$ values (nearly $1000 \mu \mathrm{V} / \mathrm{K}$ for the given carrier concentration range) while the other systems produced much smaller values $(<50 \mu \mathrm{V} / \mathrm{K})$. Also, the $\mathrm{MoX}_{2}$ and $\mathrm{WX}_{2}$ systems yield similar plots for all cases except the $n$-type $S$ calculation for the $\mathrm{MTe}_{2}$ structures (see Figure 5.2f). The $\mathrm{TiTe}_{2}$ results show a noticeable peak in the $S$ of about $450-500$ $\mu \mathrm{V} / \mathrm{K}$ for both $p$ - and $n$-type calculations. It suggests a bipolar conduction for carrier concentrations below $10^{11}$ carriers $/ \mathrm{cm}^{2}$ and an optimum $S$ value for this system. This bipolar effect could cause degradation in the thermoelectric performance. 

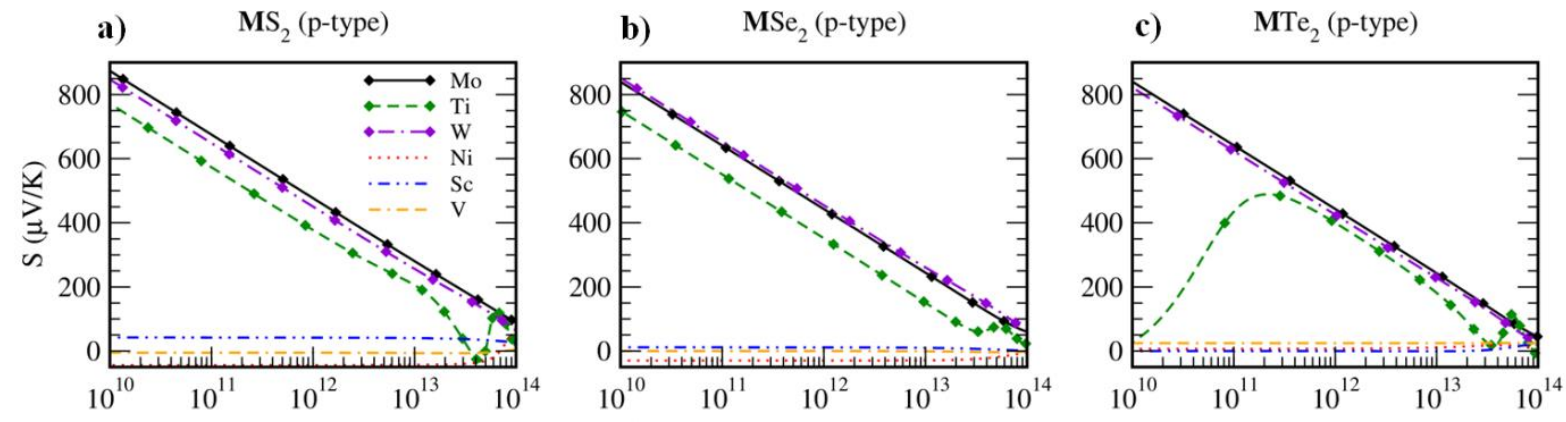

d) $\quad \mathrm{MS}_{2}$ (n-type)

e) $\quad \mathrm{MSe}_{2}$ (n-type)
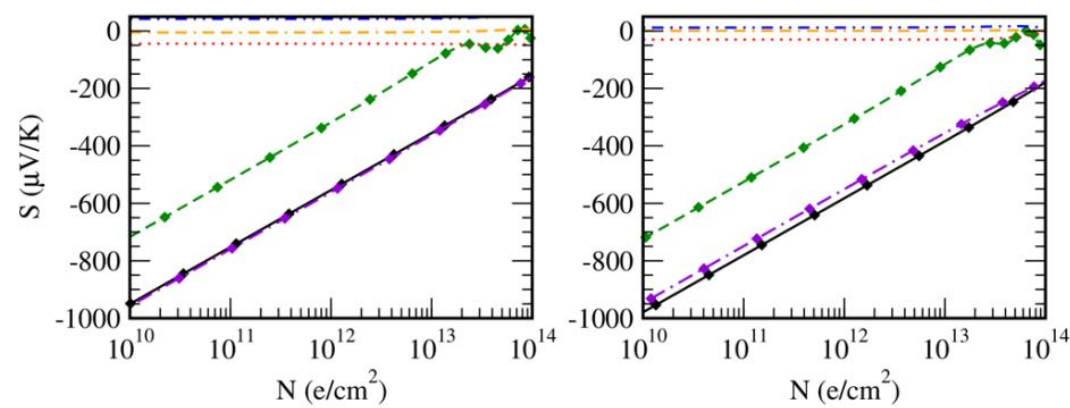

f) $\quad \mathbf{M T e}_{2}($ n-type)

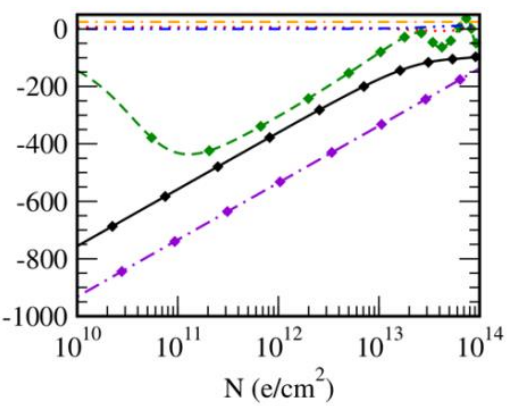

Figure 5.2. Seebeck coefficient, $S(\mu \mathrm{V} / \mathrm{K})$, calculated as a function of carrier concentration $N\left(\mathrm{e} / \mathrm{cm}^{2}\right)$ for $2 D$ TMDs. Plots (a)-(c) show the $p$-type $S$ values while plots (d)-(f) depict $n$-type values. The first (a,d), second (b, e), and third (c, f) columns compare the MS2, MSe2, and MTe2 systems, respectively. The $\mathrm{MoX}_{2}, \mathrm{TiX}_{2}$, and $\mathrm{WX}_{2}$ systems all exhibit semiconducting behavior and are distinguished by lines with diamonds (Mo diamonds-solid line, Ti diamonds-dashed line, and W diamonds-dotdashed line). The more-conductive $\mathrm{NiX}_{2}, \mathrm{ScX}_{2}$, and $\mathrm{VX}_{2}$ systems all exhibit low $S$ values and are depicted using lines (Ni dotted line, Sc dot-dot-dashed line, and V dashdash-dotted line). All calculations were performed at the temperature of $300 \mathrm{~K}$.

The $S$ values were combined with electrical conductivity $(\sigma / \tau)$ calculations, and using the constant relaxation time approximation, ${ }^{45,46}$ the $S^{2} \sigma$ values were calculated. The choice of relaxation time $(\tau)$ comes from the work of Kaasbjerg et al. ${ }^{47}$ who used DFT to calculate the effective electron mass $\left(m^{*}=0.48 m_{e}\right)$ and electron mobility in single layer $\mathrm{MoS}_{2}$. At $300 \mathrm{~K}$, and for carrier densities between $10^{11}$ and $10^{13} \mathrm{~cm}^{-2}$, they calculated an electron mobility of about $\mu=400 \mathrm{~cm}^{2} \mathrm{~V}^{-1} \mathrm{~s}^{-1}$. Using the equation $\mu=\frac{q}{m^{*}} \tau$, where $q$ is the electronic charge, the average relaxation time equates to about $\tau \approx 10^{-13} \mathrm{~s}$. This value was used for each system and the resulting first-peak maximum $S^{2} \sigma$ values are given in Table 5.2. The $n$-type results indicate a correlation between the magnitude of the 
$S^{2} \sigma$ and the mass of the constituent transition metal. The heaviest metals, W and Mo, produce the largest $S^{2} \sigma$ of $7.90 * 10^{-3}$ and $5.82 * 10^{-3} \mathrm{~W} / \mathrm{m} / \mathrm{K}^{2}$, respectively, while the lightest metal, Sc, has the smallest $S^{2} \sigma(0.78 \text { to } 1.39)^{*} 10^{-3} \mathrm{~W} / \mathrm{m} / \mathrm{K}^{2}$. The type of chalcogen does not have a large impact on the $S^{2} \sigma$ of the resultant TMD. For example, the $\mathrm{TiX}_{2}$ system has a range of only $0.04 * 10^{-3} \mathrm{~W} / \mathrm{m} / \mathrm{K}^{2}$. This could be useful for design purposes, where necessary restrictions on the use of chalcogen would likely not affect the overall performance of the material. The $p$-type $S^{2} \sigma$ results, however, are not as consistent. Many of the systems exhibit large dependence on the type of chalcogen used. For example, the $\mathrm{NiX}_{2}$ system has a range of values exceeding $7.83 * 10^{-3} \mathrm{~W} / \mathrm{m} / \mathrm{K}^{2}$.

Table 5.2 Power factor $\left(S^{2} \sigma\right)$ values for the 18 2D TMDs. Values are reported as $10^{3}$ times larger than actual for the purpose of clarity in the table. Each value, when multiplied by $10^{-3}$, yields units of $\mathrm{W} / \mathrm{m} / \mathrm{K}^{2}$. These are calculated at $300 \mathrm{~K}$ for both $n$ and $p$-type.

\begin{tabular}{|c|c|c|c|c|}
\hline \multirow[b]{2}{*}{$M$} & \multirow[b]{2}{*}{$X$} & \multirow[b]{2}{*}{ Type } & \multicolumn{2}{|c|}{$S^{2} \sigma^{*} 10^{-3}\left(\mathrm{~W} / \mathrm{m} / \mathrm{K}^{2}\right)$} \\
\hline & & & $n$-type & $p$-type \\
\hline \multirow{3}{*}{ Mo } & S & $2 \mathrm{H}$ & 5.82 & 5.09 \\
\hline & $\mathrm{Se}$ & $2 \mathrm{H}$ & 5.49 & 3.70 \\
\hline & $\mathrm{Te}$ & $2 \mathrm{H}$ & 5.26 & 3.26 \\
\hline \multirow[t]{3}{*}{$\mathrm{Ni}$} & $S$ & $1 \mathrm{~T}$ & 3.36 & 8.62 \\
\hline & $\mathrm{Se}$ & $1 \mathrm{~T}$ & 2.97 & 0.79 \\
\hline & $\mathrm{Te}$ & $2 \mathrm{H}$ & 0.45 & 3.03 \\
\hline \multirow[t]{3}{*}{$\mathrm{Sc}$} & $\mathrm{S}$ & $2 \mathrm{H}$ & 1.16 & 0.75 \\
\hline & $\mathrm{Se}$ & $2 \mathrm{H}$ & 1.39 & 0.96 \\
\hline & $\mathrm{Te}$ & $2 \mathrm{H}$ & 0.78 & 2.81 \\
\hline \multirow[t]{3}{*}{$\mathrm{Ti}$} & $S$ & $1 \mathrm{~T}$ & 2.07 & 9.32 \\
\hline & $\mathrm{Se}$ & $2 \mathrm{H}$ & 2.11 & 3.16 \\
\hline & $\mathrm{Te}$ & $2 \mathrm{H}$ & 2.10 & 6.05 \\
\hline \multirow[t]{3}{*}{ V } & $\mathrm{S}$ & $1 \mathrm{~T}$ & 1.29 & 0.86 \\
\hline & $\mathrm{Se}$ & $2 \mathrm{H}$ & 5.18 & 1.68 \\
\hline & $\mathrm{Te}$ & $2 \mathrm{H}$ & 4.25 & 0.79 \\
\hline
\end{tabular}




\begin{tabular}{|c|c|c|c|c|}
\hline $\mathrm{W}$ & $\mathrm{S}$ & $2 \mathrm{H}$ & 7.39 & 5.14 \\
\hline & $\mathrm{Se}$ & $2 \mathrm{H}$ & 7.90 & 5.05 \\
\hline & $\mathrm{Te}$ & $2 \mathrm{H}$ & 6.10 & 4.21 \\
\hline
\end{tabular}

\section{Phonon Property Screening}

A thorough understanding of heat transfer is important for device design. In particular, 2D TMDs have attracted much interest in this regard for the unique transport of thermal energy at the low dimension. Developing high-performance thermoelectric materials, for example, requires a large $Z T$ which is dependent upon having low thermal conductivity. Thermal conductivity calculations involve a detailed investigation of the Boltzmann transport equation, namely second- and third-order solutions to account for both normal and Umklapp phonon modes. Here, we focus primarily on phonon DoS calculations as a means of qualitatively comparing phonon properties for 2D TMDs in order to identify trends. Figure 5.3 shows the projected phonon DoS calculation results for the $\mathrm{MS}_{2}$ system. The total phonon DoS is shown with a solid black line in each case while contributions from the transition metal and sulfur atoms are represented by dotdashed red and dashed black lines, respectively. Figure 5.3a-f lists each $\mathrm{MS}_{2}$ in order of increasing atomic mass of the constituent transition metal. As the mass difference between transition metal and sulfur atoms $(32.066 \mathrm{u})$ increases, the frequency gap between the acoustic and optical phonon bands generally increases, suggesting that, although mass difference can be beneficial for increased phonon scattering (and therefore decreased thermal conductivity), there might be an optimum mass difference before the different modes no longer scatter one another. If the mass difference is too large, the impurity (or defect) scattering term decreases. The $\mathrm{WS}_{2}$ results, for example, have the largest mass difference (151.784 u) and consequently show the least amount of overlap 
between $\mathrm{M}$ and $\mathrm{S}$ modes. Such features imply minimal phonon scattering and thus a larger thermal conductivity, $\kappa$. It has been evidenced by Gu et al. that $\mathrm{WS}_{2}$ does indeed have a larger $\kappa$ than that of $\operatorname{MoS}_{2} \cdot{ }^{19}$ In addition, their results reveal a monotonically increasing lattice thermal conductivity with increasing acoustic-optical frequency bandgap. Sc, Ti, and $\mathrm{V}$ sulfides have negligibly small frequency gaps while $\mathrm{Ni}$, Mo, and W sulfides have substantial gaps. In this regard, $\mathrm{Sc}, \mathrm{Ti}$, and $\mathrm{V}$ sulfides should exhibit the lowest lattice thermal conductivity.

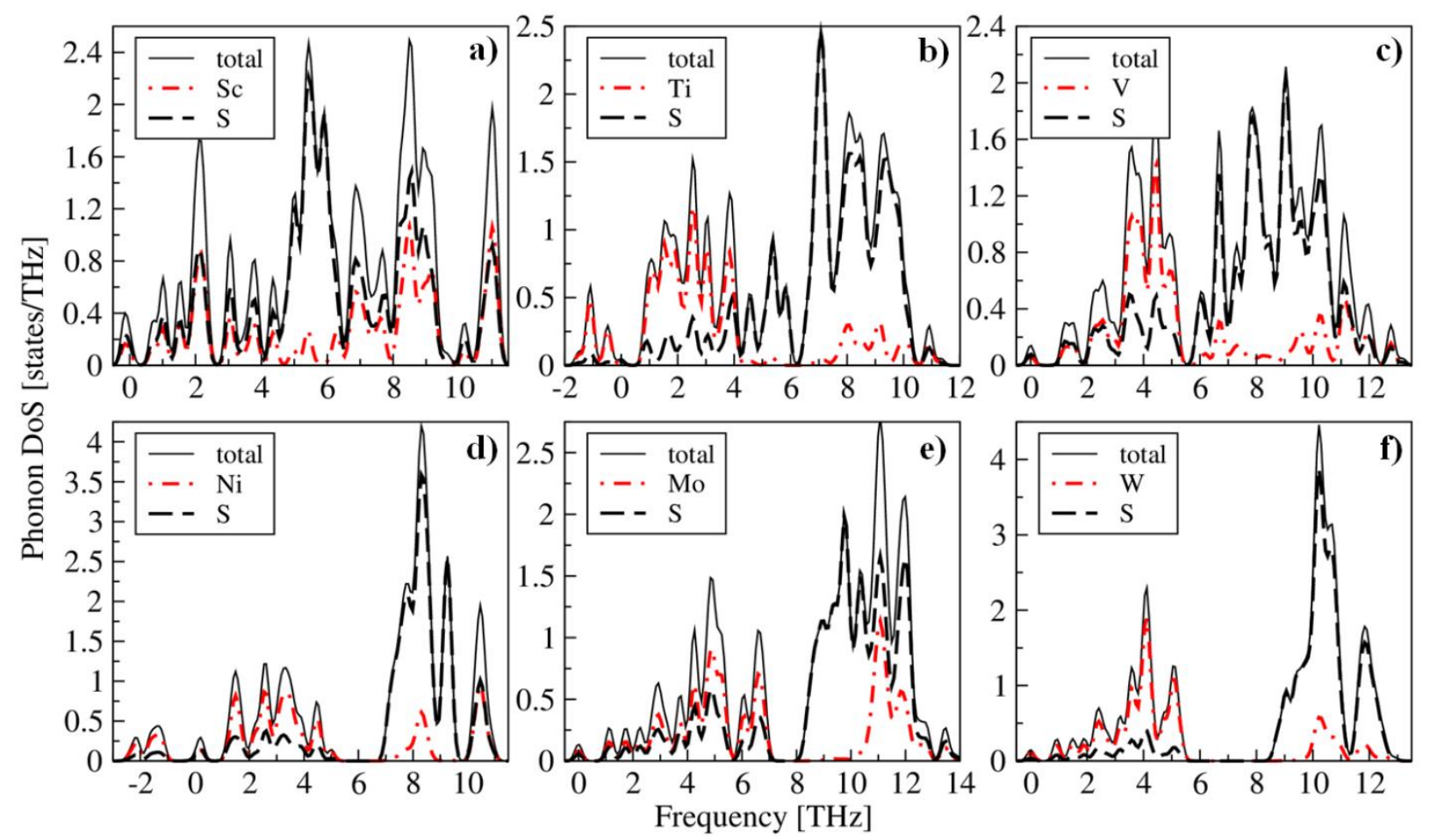

Figure 5.3. Projected phonon DoS for each member of the $\mathbf{M S}_{2}$ system. Each plot depicts the total phonon DoS with a solid line, the contribution from the transition metal with a dot-dashed line, and the contribution from the $S$ atoms with a dashed line. Plots (a)-(f) are shown in order of increasing atomic mass of the transition metal as follows (a) $\mathrm{ScS}_{2}$, (b) $\mathrm{TiS}_{2}$, (c) $\mathrm{VS}_{2}$, (d) $\mathrm{NiS}_{2}$, (e) $\mathrm{MoS}_{2}$, and (f) $\mathrm{WS}_{2}$.

\subsection{Conclusion}

A list of eighteen 2D TMD compounds, having the chemical formula $\mathrm{MX}_{2}(\mathrm{M}=$ Mo, Ni, Sc, Ti, V, and W; X = S, Se, Te), were chosen based on their potential stability 
and on the criticality scores of the constituent transition metals. First-principles geometry, electrical property, and phonon property calculations were performed. Results were compared with features of the transition metals in order to identify predictive property trends. The atomic radius of the transition metal correlates with the 2D TMD properties. While the layer thickness of the compounds increases linearly with the transition metal radius, the lattice parameter remains constant. The electronic bandgaps of TMDs follow a Gaussian-like distribution across the range of transition metal radii wherein the smallest and largest constituent metals yield negligibly small bandgaps. In contrast, the mid-sized metals yield bandgaps of greater than $1 \mathrm{eV} . \mathrm{MoX}_{2}, \mathrm{TiX}_{2}$, and $\mathrm{WX}_{2}$ compounds have the largest $S$. TiTe 2 compound exhibits bipolar conduction behavior with a noticeable $S$ peak of about 450 to $500 \mu \mathrm{V} / \mathrm{K}$ for both $p$ - and $n$-type calculations. The atomic mass of constituent metals also affects the compound properties. The heaviest metals, W and Mo, produce the largest $n$-type $S^{2} \sigma$. Mass difference between transition metal and sulfur atom enlarges the frequency gap between acoustic and optical phonon bands, indicating decreased phonon scattering. The Sc, Ti, and V sulfides have the smallest mass differences and the largest interaction between the metal and sulfur contributions to phonon DoS, so they should exhibit a lower lattice thermal conductivity than the other $\mathrm{MX}_{2}$ compounds. Our work provides a new approach to draw correlations between physical properties of constituent transition metals and resultant 2D TMDs.

Expanding on the methodology from the skutterudite work in Chapter Four, this chapter explored the calculation of semi-classic transport coefficients as another metric for investigating electrical properties. The method of calculating $S^{2} \sigma$ and $S$ was employed in Chapters Six to further study dopant effects. 


\section{Acknowledgements}

The authors would like to thank Dr. Dave Estrada at Boise State for helpful discussions of the experiment, Ken Blair at Boise State for assistance with computing facilities, as well as Idaho National Laboratory's high performance computing (HPC) center for computing support. 


\section{References}

(1) Ogawa, S. Magnetic-Properties of 3d Transition-Metal Dichalcogenides with the Pyrite Structure. J Appl Phys 1979, 50, 2308.

(2) Shishidou, T.; Freeman, A. J.; Asahi, R. Effect of GGA on the halfmetallicity of the itinerant ferromagnet CoS2. Phys Rev B 2001, 64, 180401.

(3) Tributsch, H. Solar Energy-Assisted Electrochemical Splitting of Water. Z Naturforsch A 1977, 32, 972.

(4) Coleman, J. N.; Lotya, M.; O'Neill, A.; Bergin, S. D.; King, P. J.; Khan, U.; Young, K.; Gaucher, A.; De, S.; Smith, R. J.; Shvets, I. V.; Arora, S. K.; Stanton, G.; Kim, H. Y.; Lee, K.; Kim, G. T.; Duesberg, G. S.; Hallam, T.; Boland, J. J.; Wang, J. J.; Donegan, J. F.; Grunlan, J. C.; Moriarty, G.; Shmeliov, A.; Nicholls, R. J.; Perkins, J. M.; Grieveson, E. M.; Theuwissen, K.; McComb, D. W.; Nellist, P. D.; Nicolosi, V. Two-Dimensional Nanosheets Produced by Liquid Exfoliation of Layered Materials. Science 2011, 331, 568.

(5) Ataca, C.; Sahin, H.; Ciraci, S. Stable, Single-Layer MX2 TransitionMetal Oxides and Dichalcogenides in a Honeycomb-Like Structure. Journal of Physical Chemistry C 2012, 116, 8983.

(6) Rasmussen, F. A.; Thygesen, K. S. Computational 2D Materials Database: Electronic Structure of Transition-Metal Dichalcogenides and Oxides. Journal of Physical Chemistry C 2015, 119, 13169.

(7) Butler, S. Z.; Hollen, S. M.; Cao, L. Y.; Cui, Y.; Gupta, J. A.; Gutierrez, H. R.; Heinz, T. F.; Hong, S. S.; Huang, J. X.; Ismach, A. F.; Johnston-Halperin, E.; Kuno, M.; Plashnitsa, V. V.; Robinson, R. D.; Ruoff, R. S.; Salahuddin, S.; Shan, J.; Shi, L.; Spencer, M. G.; Terrones, M.; Windl, W.; Goldberger, J. E. Progress, Challenges, and Opportunities in Two-Dimensional Materials Beyond Graphene. Acs Nano 2013, 7, 2898.

(8) McDonnell, S.; Addou, R.; Buie, C.; Wallace, R. M.; Hinkle, C. L. DefectDominated Doping and Contact Resistance in MoS2. Acs Nano 2014, 8, 2880. 
(9) Gong, C.; Colombo, L.; Wallace, R. M.; Cho, K. The Unusual Mechanism of Partial Fermi Level Pinning at Metal-MoS2 Interfaces. Nano Lett 2014, 14, 1714.

(10) Ganatra, R.; Zhang, Q. Few-Layer MoS2: A Promising Layered Semiconductor. Acs Nano 2014, 8, 4074.

(11) Radisavljevic, B.; Radenovic, A.; Brivio, J.; Giacometti, V.; Kis, A. Single-layer MoS2 transistors. Nat Nanotechnol 2011, 6, 147.

(12) Lopez-Sanchez, O.; Lembke, D.; Kayci, M.; Radenovic, A.; Kis, A. Ultrasensitive photodetectors based on monolayer MoS2. Nat Nanotechnol 2013, $8,497$.

(13) Baugher, B. W. H.; Churchill, H. O. H.; Yang, Y. F.; Jarillo-Herrero, P. Optoelectronic devices based on electrically tunable p-n diodes in a monolayer dichalcogenide. Nat Nanotechnol 2014, 9, 262.

(14) Chen, W.; Santos, E. J. G.; Zhu, W. G.; Kaxiras, E.; Zhang, Z. Y. Tuning the Electronic and Chemical Properties of Monolayer MoS2 Adsorbed on Transition Metal Substrates. Nano Lett 2013, 13, 509.

(15) Jaramillo, T. F.; Jorgensen, K. P.; Bonde, J.; Nielsen, J. H.; Horch, S.; Chorkendorff, I. Identification of active edge sites for electrochemical H-2 evolution from MoS2 nanocatalysts. Science 2007, 317, 100.

(16) Huang, W.; Da, H. X.; Liang, G. C. Thermoelectric performance of MX2 (M = Mo, W; X = S, Se) monolayers. J Appl Phys 2013, 113, 104304.

(17) Huang, W.; Luo, X.; Gan, C. K.; Quek, S. Y.; Liang, G. C. Theoretical study of thermoelectric properties of few-layer MoS2 and WSe2. Physical Chemistry Chemical Physics 2014, 16, 10866.

(18) Lee, C.; Hong, J.; Whangbo, M. H.; Shim, J. H. Enhancing the Thermoelectric Properties of Layered Transition-Metal Dichalcogenides 2HMQ(2) (M = Mo, W; Q = S, Se, Te) by Layer Mixing: Density Functional Investigation. Chem Mater 2013, 25, 3745. 
(19) Gu, X. K.; Yang, R. G. Phonon transport in single-layer transition metal dichalcogenides: A first-principles study. Appl Phys Lett 2014, 105, 131903.

(20) Nolas, G. S.; Slack, G. A. Thermoelectric clathrates. Am Sci 2001, 89, 136.

(21) Zou, M. M.; Li, J. F.; Kita, T. Thermoelectric properties of fine-grained FeVSb half-Heusler alloys tuned to p-type by substituting vanadium with titanium. Journal of Solid State Chemistry 2013, 198, 125.

(22) Dyck, J. S.; Chen, W. D.; Uher, C.; Chen, L.; Tang, X. F.; Hirai, T. Thermoelectric properties of the n-type filled skutterudite Ba0.3Co4Sb12 doped with Ni. J Appl Phys 2002, 91, 3698.

(23) Yang, J.; Zhang, W.; Bai, S. Q.; Mei, Z.; Chen, L. D. Dual-frequency resonant phonon scattering in BaxRyCo4Sb12 (R=La, Ce, and Sr). Appl Phys Lett 2007, 90, 192111.

(24) Williamson, I.; Her, L. J. Y.; Su, X. L.; Yan, Y. G.; Wong-Ng, W.; Li, L. Improved thermoelectric performance of $(\mathrm{Fe}, \mathrm{Co}) \mathrm{Sb}$-3-type skutterudites from first-principles. J Appl Phys 2016, 119, 055101.

(25) Tan, G. J.; Wang, S. Y.; Li, H.; Yan, Y. G.; Tang, X. F. Enhanced thermoelectric performance in zinc substituted p-type filled skutterudites CeFe4xZnxSb12. Journal of Solid State Chemistry 2012, 187, 316.

(26) Hicks, L. D.; Dresselhaus, M. S. Effect of Quantum-Well Structures on the Thermoelectric Figure of Merit. Phys Rev B 1993, 47, 12727.

(27) Hicks, L. D.; Dresselhaus, M. S. Thermoelectric Figure of Merit of a OneDimensional Conductor. Phys Rev B 1993, 47, 16631.

(28) Mahan, G. D.; Sofo, J. O. The best thermoelectric. P Natl Acad Sci USA 1996, 93, 7436.

(29) Hochbaum, A. I.; Chen, R. K.; Delgado, R. D.; Liang, W. J.; Garnett, E. C.; Najarian, M.; Majumdar, A.; Yang, P. D. Enhanced thermoelectric performance of rough silicon nanowires. Nature 2008, 451, 163. 
(30) Boukai, A. I.; Bunimovich, Y.; Tahir-Kheli, J.; Yu, J. K.; Goddard, W. A.; Heath, J. R. Silicon nanowires as efficient thermoelectric materials. Nature 2008, $451,168$.

(31) Graedel, T. E.; Harper, E. M.; Nassar, N. T.; Nuss, P.; Reck, B. K. Criticality of metals and metalloids. P Natl Acad Sci USA 2015, 112, 4257.

(32) Blöchl, P. E. Projector augmented-wave method. Phys Rev B 1994, 50, 17953.

(33) Kresse, G.; Joubert, D. From ultrasoft pseudopotentials to the projector augmented-wave method. Phys Rev B 1999, 59, 1758.

(34) Kresse, G.; Furthmuller, J. Efficient iterative schemes for ab initio totalenergy calculations using a plane-wave basis set. Phys Rev B 1996, 54, 11169.

(35) Perdew, J. P.; Burke, K.; Ernzerhof, M. Generalized gradient approximation made simple. Phys Rev Lett 1996, 77, 3865.

(36) Liechtenstein, A. I.; Anisimov, V. I.; Zaanen, J. Density-functional theory and strong interactions: Orbital ordering in Mott-Hubbard insulators. Phys Rev B 1995, 52, R5467.

(37) Parlinski, K.; Li, Z. Q.; Kawazoe, Y. First-principles determination of the soft mode in cubic ZrO2. Phys Rev Lett 1997, 78, 4063.

(38) Togo, A.; Oba, F.; Tanaka, I. First-principles calculations of the ferroelastic transition between rutile-type and $\mathrm{CaCl}(2)$-type $\mathrm{SiO}(2)$ at high pressures. Phys Rev B 2008, 78, 134106.

(39) Grimme, S. Semiempirical GGA-type density functional constructed with a long-range dispersion correction. J Comput Chem 2006, 27, 1787.

(40) Boker, T.; Severin, R.; Muller, A.; Janowitz, C.; Manzke, R.; Voss, D.; Kruger, P.; Mazur, A.; Pollmann, J. Band structure of MoS2, MoSe2, and alphaMoTe2: Angle-resolved photoelectron spectroscopy and ab initio calculations. Phys Rev B 2001, 64, 235305. 
(41) Mak, K. F.; Lee, C.; Hone, J.; Shan, J.; Heinz, T. F. Atomically Thin MoS2: A New Direct-Gap Semiconductor. Phys Rev Lett 2010, 105, 136805.

(42) Sansonetti, J. E.; Martin, W. C.; July 1, 2009 ed.; National Institute of Standards and Technology: http://www.nist.gov/pml/data/handbook/, 2014.

(43) Shannon, R. D. Revised Effective Ionic-Radii and Systematic Studies of Interatomic Distances in Halides and Chalcogenides. Acta Crystallogr A 1976, 32, 751.

(44) Madsen, G. K. H.; Singh, D. J. BoltzTraP. A code for calculating bandstructure dependent quantities. Computer Physics Communications 2006, 175, 67.

(45) Wang, Y.; Chen, X.; Cui, T.; Niu, Y. L.; Wang, Y. C.; Wang, M.; Ma, Y. M.; Zou, G. T. Enhanced thermoelectric performance of PbTe within the orthorhombic Pnma phase. Phys Rev B: Condens. Matter Mater. Phys. 2007, 76, 155127.

(46) Wang, C.; Wang, Y. X.; Zhang, G. B.; Peng, C. X. Electronic Structure and Thermoelectric Properties of ZnO Single-Walled Nanotubes and Nanowires. Journal of Physical Chemistry C 2013, 117, 21037.

(47) Kaasbjerg, K.; Thygesen, K. S.; Jacobsen, K. W. Phonon-limited mobility in n-type single-layer MoS2 from first principles. Phys Rev B 2012, 85, 115317. 


\section{CHAPTER SIX: IMPROVEMENT OF 2D TMD MATERIALS THROUGH METAL-} SITE SUBSTITUTIONAL DOPANTS*

This chapter is comprised of two works: the first is published by Elsevier in the Chemical Physics Letters and the second is submitted for publication in the Journal of Applied Physics. These works should be referenced appropriately.

\section{References:}

I. Williamson, S. Li, A. Correa Hernandez, M. Lawson, Y. Chen, L. Li, "Structural, electrical, phonon, and optical properties of Ti- and V-doped two-dimensional $\mathrm{MoS}_{2}$." Chemical Physics Letters. 674157 (2017).

Reproduced by permission of Elsevier and hosted on Science Direct at <http://www.sciencedirect.com/science/article/pii/S0009261417301707>.

I. Williamson, N. Wilson, M. Lawson, S. Li, Y. Chen, L. Li, “Computational screening of metal-site dopants in two-dimensional $\mathrm{MX}_{2}(\mathrm{M}=\mathrm{Mo}, \mathrm{W} ; \mathrm{X}=\mathrm{S}, \mathrm{Se}, \mathrm{Te})$. . Journal of Applied Physics. submitted (2017).

*This chapter includes modifications from the originally published versions. Optical properties calculations that were included in both works (contributed by Shasha Li and Dr. Yue Chen at The University of Hong Kong) have been omitted from this dissertation. 
Structural, electrical, phonon, and optical properties of Ti- and V-doped twodimensional $\mathrm{MoS}_{2}$

$\begin{gathered}\text { Izaak Williamson } \\ \text { Shasha } \mathrm{Li}^{\mathrm{b}}\end{gathered}$
Andres Correa Hernandez
Matthew Lawson
Yue Chen

Published in:

Chemical Physics Letters

February 2017

${ }^{a}$ Micron School of Materials Science and Engineering, Boise State University, Boise, ID 83725 .

${ }^{b}$ Department of Mechanical Engineering, The University of Hong Kong, Pokfulam Road, Hong Kong SAR, China.

${ }^{c}$ Center for Advanced Energy Studies, Idaho Falls, ID 83401. 
Computational screening of metal-site dopants in two-dimensional $\mathrm{MX}_{2}(\mathrm{M}=\mathrm{Mo}$, $\mathrm{W} ; \mathrm{X}=\mathrm{S}, \mathrm{Se}, \mathrm{Te})$

Izaak Williamson $^{\mathrm{a}}$

Nate Wilson ${ }^{\mathrm{b}}$

Matthew Lawson ${ }^{\mathrm{a}}$

Shasha $\mathrm{Li}^{\mathrm{c}}$

Yue Chen ${ }^{\mathrm{c}}$

Lan $\mathrm{Li}^{\mathrm{a}, \mathrm{d}}$

Submitted for Publication in:

The Journal of Applied Phyics

March 2017

${ }^{a}$ Micron School of Materials Science and Engineering, Boise State University, Boise, ID 83725 .

${ }^{b}$ Department of Chemical Engineering and Materials Science, University of Minnesota, Minneapolis, MN 55455.

${ }^{c}$ Department of Mechanical Engineering, The University of Hong Kong, Hong Kong SAR, China.

${ }^{d}$ Center for Advanced Energy Studies, 
Idaho Falls, ID 83401.

NOTICE: This is the author's version of a work that was submitted for publication in the Journal of Applied Physics. Changes resulting from the publishing process, such as peer review, editing, corrections, structural formatting, and other quality control mechanisms will not be reflected in this document. Changes may have been made to this work since it was submitted for publication. 


\begin{abstract}
2D TMDs, having the chemical formula $\mathrm{MX}_{2}(\mathrm{M}=$ transition metal, $\mathrm{X}=$ chalcogen), have received a lot of attention recently for their unique properties and potential for materials-by-design applications. Having notable electrical, magnetic, optical, and thermoelectric properties, 2D TMDs can be further tuned through the implementation of substitutional dopants. This work utilizes first-principles DFT calculations to explore the effects of metal-site dopant concentration and type on structural, energetic, electrical, transport, and phonon properties. These effects decrease with concentration until they converge at a dopant concentration of 2.083 at. $\%$, where the dopants are effectively isolated. Electronic bandgap calculations reveal W-doped $\mathrm{MoX}_{2}$ and Mo-doped $\mathrm{WX}_{2}$ to be among the only observed semiconductors while Boltzmann transport properties confirm that these six structures have the largest power factors. This work offers an extensive investigation of metal-site dopants and how their concentration and type impact 2D TMD materials, identifying factors for tailoring their performance.
\end{abstract}

\title{
6.1 Introduction
}

There are a variety of potential uses for transition metal dichalcogenides (TMDs) stemming from their unique and tunable properties. Recently, these materials have attracted much interest due to novel magnetic, ${ }^{1,2}$ electrical, ${ }^{3-7}$ thermoelectric, ${ }^{8-11}$ and optical $^{7,12-14}$ properties. The structure of a TMD is a large contributor to the material's versatility and wide range of interesting properties. The chemical formula is $\mathrm{MX}_{2}$ where $\mathrm{M}$ is a transition metal and $\mathrm{X}$ is a chalcogen. The structure is composed of offset stacked layers where each layer is made up of $\mathrm{M}$ atoms sandwiched between two $\mathrm{X}$ sheets. For the $2 \mathrm{H}$-type $\mathrm{MX}_{2}$ system, it is arranged such that $D_{6 h}$ point group symmetry exists about 
the $\mathrm{M}$ atoms. The layers are held together through strong covalent bonds while the forces between the layers are much weaker van der Waals interactions, much like that of graphite and graphene. Through mechanical exfoliation, ${ }^{15}$ the layers can be separated into few-layer or single-layer structures. These nanostructures offer even more interesting opportunities for materials-by-design exploration.

A large advantage for two-dimensional (2D) $\mathrm{MX}_{2}$ structures comes from the reduced dimensionality which can extend the limitations of bulk TMD properties. In field-effect transistors (FETs), for example, the smaller device features have greatly reduced short-channel effects, reduced switching voltage, and increased the on-off ratio. ${ }^{5,12}$ Another example of significant changes in the electronic structure comes in the case of $\mathrm{MoS}_{2}$ which undergoes a transition from having an indirect bandgap of $1.2 \mathrm{eV}$ in bulk $^{16}$ to a direct bandgap of $1.8 \mathrm{eV}$ in $2 \mathrm{D} .{ }^{17}$ This effect is useful for electronic and optoelectronic applications. ${ }^{18}$ Under the photoexcitation condition, the direct bandgap semiconductor would have a high absorption coefficient and efficient electron-hole pair generation, suggesting promising application as photonic and optoelectronic devices. For example, photoluminescence has been found to increase with decreasing thickness of $\mathrm{MoS}_{2},{ }^{19}$ and the luminescence quantum efficiency of a freestanding monolayer is four orders of magnitude stronger than in its bulk counterpart. ${ }^{17}$ The $2 \mathrm{D} \mathrm{MoS}_{2}$ was also reported as ultrasensitive phototransistors because of the improved device mobility and on current. ${ }^{13}$ Furthermore, the photoresponsivity of $2 \mathrm{D} \mathrm{MoS}_{2}$ phototransistors was found to exceed that of a graphene-based device. ${ }^{20}$ Additionally, the quantum confinement effect at lower dimensionalities enhances thermoelectric properties by increasing the Seebeck coefficient, $S$, (the measure of a material's voltage response to temperature 
change) where the thermoelectric performance is determined using the figure of merit, $Z T$ (see Equation 2.1). ${ }^{21-23}$. Another factor that contributes to thermoelectric enhancement in 2D materials is the increase in power factor, $S^{2} \sigma$, due to the change in shape of the density of modes (DoM). ${ }^{24,25}$ Through increased diffusive boundary scattering, it is expected that the thermal conductivity could decrease, further enhancing thermoelectric properties. ${ }^{26,27}$ Such a large potential for properties and transport behavior emphasizes the need to fully understand $2 \mathrm{D} \mathrm{MX}$ materials.

A number of screening methods have been implemented through first-principles density functional theory (DFT) calculations in order to identify more favorable $\mathrm{MX}_{2}$ compositions and potentially highlight a narrower list of promising candidates. The works of Ataca et al. ${ }^{3}$ and Rasmussen $e t$ al. ${ }^{4}$ have explored various compositions, focusing on stability, electrical, and optical properties. Our previous work ${ }^{28}$ combined these findings with transition metal "criticality scores" 29 in order to highlight the environmental impact and materials availability as well as the more stable and promising compositions. Our narrowed list of eighteen $2 \mathrm{D} \mathrm{MX}_{2}$ structures $(\mathrm{M}=\mathrm{Mo}, \mathrm{Ni}, \mathrm{Sc}, \mathrm{Ti}, \mathrm{V}$, and $\mathrm{W} ; \mathrm{X}=\mathrm{S}, \mathrm{Se}$, and $\mathrm{Te}$ ) was then investigated for structural, electrical, and phonon property effects. This identified trends in the structure-property relationship which can lead to the identification of novel compositions and facilitate materials design.

Further property improvements and tuning can be achieved through the use of metal-site substitutional dopants which yield interesting changes in the intrinsic properties of 2D TMDs. These dopants can cause enhancements such as inducing magnetism ${ }^{30-33}$ and tuning of the electrical conductivity ${ }^{33}$ They also offer the ability to modify the bandgap and photoluminescence ${ }^{34}$ and control whether it is a $p$-or $n$-type 
semiconductor. ${ }^{35}$ Previous work has shown that these dopants are stable in 2D TMDs and that they are a substitutional dopant rather than an interstitial impurity. ${ }^{36-39}$ This type of defect tends to be more stable because the dopant inherits the strong covalent bonds within the TMD sheet.

In this work, we investigate the effect of dopant concentration and type in $2 \mathrm{D}$ TMD materials. We investigated substitutional Ti dopants on Mo sites in 2D $\mathrm{MoS}_{2}$ at concentrations of $0.926,1.333,2.083,3.704$, and 8.333 at.\% Ti (or 2.78, 4.0, 6.25, 11.11, and 25.0 Mo-site \% Ti) to determine its effect on various properties. Next, six different transition metals $\left(\mathrm{M}^{\prime}=\mathrm{Mo}, \mathrm{Ni}, \mathrm{Sc}, \mathrm{Ti}, \mathrm{V}\right.$, and $\left.\mathrm{W}\right)$ were incorporated at the metal-site in $\mathrm{MoX}_{2}$ and $\mathrm{WX}_{2}(\mathrm{X}=\mathrm{S}, \mathrm{Se}$, or Te$)$, according to the chemical formula $\mathrm{M}^{\prime} \mathrm{M}_{15} \mathrm{X}_{32}$. These six were chosen based on the screening criteria of previous work. ${ }^{28}$ The structural, energetic, electrical, transport, and phonon properties were analyzed with respect to the physical features of the dopant atoms (e.g., atomic radius, preferred oxidation state) to better understand the doping effects of these 2D TMD structures. This approach offers a means of extensively analyzing the effects of dopant concentration and type in $\mathrm{MoX}_{2}$ and $\mathrm{WX}_{2}$ to identify key factors in improving novel 2D TMD materials.

\subsection{Computational Methods}

All calculations were performed within DFT implemented via the VASP code. ${ }^{40}$ The PBE formalism was employed with spin-polarized GGA. ${ }^{41}$ PAW pseudopotentials were used to expand the plane-wave basis set to a cutoff energy of $500 \mathrm{eV} \cdot{ }^{42,43}$ For the Brillouin zone integration, a $\Gamma$-centered $12 \times 12 \times 1$ mesh was used for the 2x $2 \times 1$ supercell (corresponding to 8.333 at.\% Ti). All other structures used $k$-point meshes proportional to this depending on their supercell size (rounded to the nearest integer). Fermi surface 
broadening was handled using a Gaussian smearing of $0.05 \mathrm{eV}$. Geometries were relaxed until residual forces were reduced to $0.01 \mathrm{eV} / \AA$. To minimize interlayer interaction, $\mathrm{MX}_{2}$ single-layer sheets in the neighboring cells were separated by a distance of at least $20 \AA$.

Electronic structure calculations were performed with denser $k$-point meshes (doubled in $\mathrm{x}$ and $\mathrm{y}$ directions with respect to the geometry optimization calculations). For the systems with dopant concentrations of 2.083 at.\% $\left(\mathrm{M}^{\prime} \mathrm{Mo}_{15} \mathrm{~S}_{32}\right)$, a denser $24 \times 24 \mathrm{x} 1$ k-point mesh was used. The DFT+U scheme ${ }^{44}$ was also implemented to account for strong correlation effects of the transition metals. Convergence-tested values for the onsite Coulomb potentials of $\mathrm{U}=4.38,8.0,3.0,8.0,6.0$, and $8.0 \mathrm{eV}$ were used for $\mathrm{Mo}, \mathrm{Ni}$, $\mathrm{Sc}, \mathrm{Ti}, \mathrm{V}$, and $\mathrm{W}$, respectively while an on-site exchange potential of $\mathrm{J}=1.0 \mathrm{eV}$ was used for all metals. The resulting band energies were used to calculate semi-classic transport coefficients using the BoltzTraP software package. ${ }^{45}$

Phonon structure calculations were performed following the method of ParlinskiLi-Kawazoe where second-order force constants were determined using DFPT. ${ }^{46,47}$ Convergence tests indicated that long-range phonon effects were adequately accounted for by means of the following supercells: $3 \times 3 \times 1,3 \times 3 \times 1,2 \times 2 \times 1$, and $2 \times 2 \times 1$ for the dopant concentrations of $0,8.333,3.704$, and 2.083 at. $\%$, respectively. The 1.333 and 0.926 at.\% systems were found to be sufficiently large to contain long-range effects without the need of further supercell sizes. The $k$-point meshes were likewise adjusted based on supercell sizes for the systems.

Error mitigation was handled for the DFT input parameters through convergence tests similar to those performed in Sections 4.2 and 5.2. The supercell sizes were chosen to represent various dopant concentrations and their affects are discussed in the following 
section. Unlike Sections 4.2 and 5.2, the work of metal-site substitution is relatively new and there were no literature values to compare with at the time the work was conducted. The structures in this chapter initially came from the pure 2D TMD screening work in Chapter Five. The error handling in Section 5.2 helped minimize errors in this chapter.

\subsection{Results}

$\underline{\text { Structure }}$

\section{Dopant Concentration}

In order to determine the substitution concentration effect, we incorporated $\mathrm{Ti}$ substitution with 8.333 at.\% Ti (i.e., $\left.\mathrm{Ti}_{1} \mathrm{Mo}_{3} \mathrm{~S}_{8}\right), 3.704$ at.\% $\mathrm{Ti}\left(\mathrm{Ti}_{1} \mathrm{Mo}_{8} \mathrm{~S}_{18}\right), 2.083$ at.\% Ti $\left(\mathrm{Ti}_{1} \mathrm{Mo}_{15} \mathrm{~S}_{32}\right), 1.333$ at.\% $\mathrm{Ti}\left(\mathrm{Ti}_{1} \mathrm{Mo}_{24} \mathrm{~S}_{50}\right)$, and 0.926 at.\% $\mathrm{Ti}\left(\mathrm{Ti}_{1} \mathrm{Mo}_{35} \mathrm{~S}_{72}\right)$. The presence of Ti dopants in $2 \mathrm{D} \mathrm{MoS}_{2}$ causes minimal structure distortion. An example of the doped 2D TMD structure is depicted in Figure 6.1.

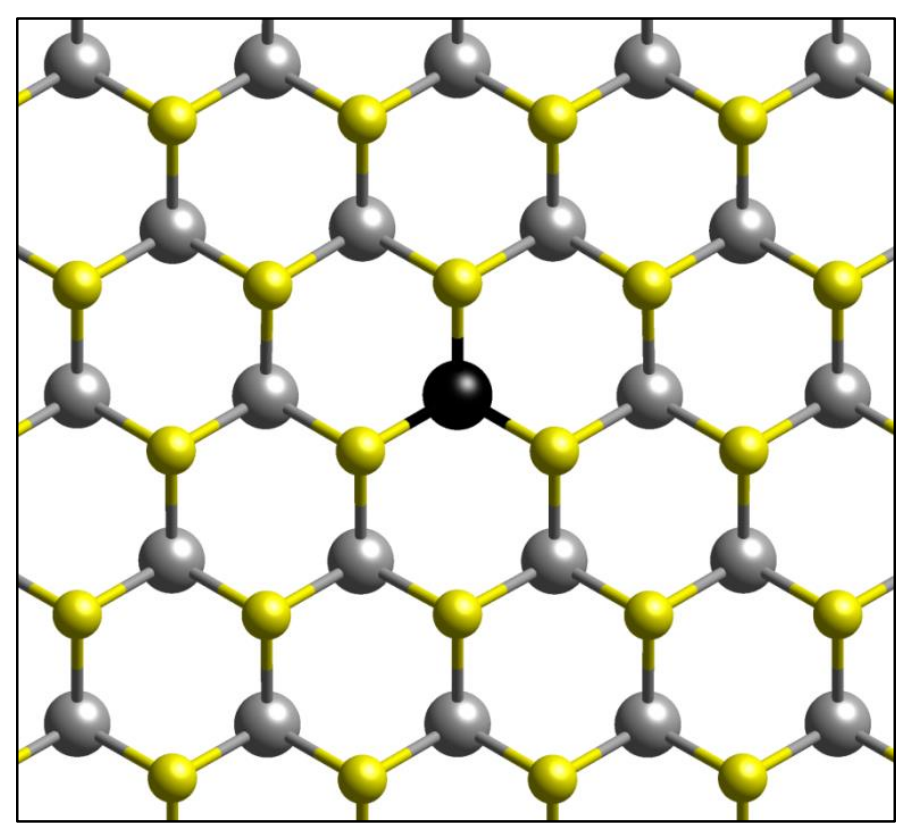

Figure 6.1. Top-down view of $M^{\prime}$ '-doped $\mathrm{MX}_{2}$ where the $M^{\prime}$ dopant atom is depicted with a black sphere, the $M$ atoms are gray spheres, and the $X$ chalcogens are yellow spheres. 
The value $a_{u c}$ (shown in Table 6.1) represents the average lattice parameter with respect to the unit cell of pure $2 \mathrm{D} \mathrm{MoS}_{2}$ and increases with Ti concentration. This minor expansion suggests the presence of small compressive forces around the Ti dopant. To further investigate this distortion, the M-S and S-S bond lengths were also measured, given as $d_{M-S}$ and $d_{S-S}$, respectively (see Figure 6.2). Note that the $\mathrm{S}-\mathrm{S}$ distance value is equivalent to the sheet thickness. The superscript ${ }^{\mathrm{M}}$ 'indicates values taken near the dopant atom, while the superscript ${ }^{\text {Mo }}$ indicates those taken about the Mo atoms farthest from the dopant. For each bond distance calculated, there is less than $1 \%$ difference between any of the doped structures. The S-M-S bond angle is given as $\theta$ and its values differ by no more than $2 \%$. As Ti concentration decreases, each of the ${ }^{\text {Mo }}$ values approach that of the pure $\mathrm{MoS}_{2}$. For instance, the $\mathrm{d}_{\mathrm{S}-\mathrm{S}}^{\mathrm{Mo}}$ and $\theta^{\mathrm{Mo}}$ values for the high concentration system (8.333 at.\% Ti) are much smaller than pure $\mathrm{MoS}_{2}$ values but increase with decreasing concentration until convergence is reached by 2.083 at.\% Ti. Likewise, the $\mathrm{d}_{\mathrm{Mo}-\mathrm{S}}^{\mathrm{Mo}}$ value for the high concentration system is larger than pure $\mathrm{MoS}_{2}$ and decreases with concentration until converging at 2.083 at.\% Ti. This means that for this concentration, the area between neighboring dopants is sufficiently large for the surrounding $\mathrm{MoS}_{2}$ structure to mimic the undoped structure, effectively isolating the dopants from each other. Also, the $\mathrm{a}_{\mathrm{uc}}$ and $\mathrm{d}_{\mathrm{Mo}-\mathrm{S}}^{\mathrm{Mo}}$ values increase with Ti concentration while the $d_{S-S}^{\text {Mo }}$ and $\theta^{\text {Mo }}$ values decrease. The expansion in the $\mathrm{x}$ - and $\mathrm{y}$-directions and compression in the z-direction indicate anisotropic strain. This could be a result of the difference in oxidation state between the Mo and Ti metals. 
Table 6.1. Geometric structure results for pure and $M^{\prime}$-doped $\left(M^{\prime}=T i\right) 2 D$ $\mathrm{MoS}_{2}$. The average lattice parameter with respect to the original unit cell, $a_{u c}$, the M-S and S-S bond distances, $d_{M-S}$ and $d_{S-S}, S-M-S$ bond angles, $\theta$, and binding energies, $E_{b}$, are given. All distances are given in angstroms $(\AA)$ and all angles are given in degrees $\left({ }^{\circ}\right)$. Superscripts ${ }^{M}$ and ${ }^{M o}$ indicate values taken at the dopant site and at the Mo site farthest from the defect, respectively.

\begin{tabular}{|c|c|c|c|c|c|c|c|c|c|}
\hline & at.\% M' & $a_{u c}$ & $d_{M^{\prime}-S}^{M^{\prime}}$ & $d_{M o-S}^{M o}$ & $d_{S-S}^{M^{\prime}}$ & $d_{S-S}^{M o}$ & $\theta^{M^{\prime}}$ & $\theta^{M o}$ & $E_{b}(\mathrm{eV})$ \\
\hline $\begin{array}{c}\text { pure } \\
\mathrm{MoS}_{2}\end{array}$ & 0 & 3.182 & -- & 2.413 & -- & 3.128 & -- & 80.81 & -- \\
\hline & 0.926 & 3.191 & 2.428 & 2.414 & 3.056 & 3.128 & 78.00 & 80.79 & -7.45 \\
\cline { 2 - 10 } & 1.333 & 3.196 & 2.428 & 2.413 & 3.055 & 3.129 & 77.98 & 80.84 & -7.45 \\
\cline { 2 - 10 } & 2.083 & 3.203 & 2.428 & 2.413 & 3.056 & 3.127 & 78.00 & 80.80 & -7.44 \\
\cline { 2 - 9 } doped & 3.704 & 3.216 & 2.429 & 2.414 & 3.057 & 3.103 & 77.99 & 79.97 & -7.31 \\
\cline { 2 - 9 } & 8.333 & 3.236 & 2.420 & 2.429 & 3.060 & 3.098 & 78.43 & 79.25 & -6.94 \\
\hline
\end{tabular}

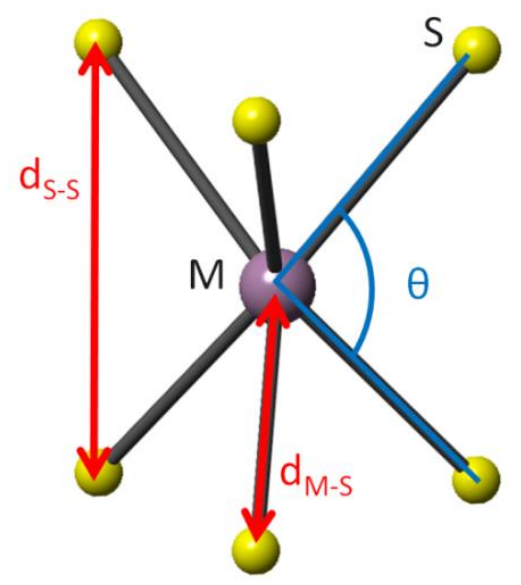

Figure 6.2. Schematic of an $\mathrm{MS}_{2}$ octahedron where the violet and yellow spheres represent $M$ and $S$ atoms, respectively. In this work, the $M$ atom depicts either the dopant, M' (Ti or V), or the constituent, Mo. The bond lengths are represented by red arrows where ds-s and dM-s depict the distance between two $S$ atoms and between the $M$ and $S$ atoms, respectively. The S-M-S bond angle is represented by the blue lines and the symbol $\theta$. 
In order to compare defect stability and confirm the structure convergence for the Ti-doped system, the binding energy $\left(E_{b}\right)$ was calculated according to the following equation:

$$
E_{b}=E_{\text {tot }}^{\text {doped }}-E_{\text {no } \mathrm{M}^{\prime}}^{\text {undoped }}-E_{\mathrm{M}^{\prime}}
$$

Here, the symbol $E_{\text {tot }}^{\text {doped }}$ represents the total energy of the doped system, $E_{n o \mathrm{M}^{\prime}}^{\text {undoped }}$ represents the energy of the undoped system where the dopant atom, $\mathrm{M}^{\prime}$, was removed, and $E_{\mathrm{M}^{\prime}}$ is the chemical potential of the dopant taken from its unary bulk structure. Our approach is similar to that of Zhou et al. ${ }^{30}$ except they also account for the strain energy associated with the dopant-MoS 2 bonds. Our $E_{n o \mathrm{M}^{\prime}}^{\text {undoped }}$ term is not relaxed, so the resulting $E_{b}$ only accounts for the energy associated with the $\mathrm{M}^{\prime}-\mathrm{S}$ bond. Furthermore, our $E_{\mathrm{M}^{\prime}}$ term is taken from the bulk $\mathrm{M}^{\prime}$ system rather than isolated $\mathrm{M}^{\prime}$. These results are given in Table 6.1. The system with the highest $E_{b}$ (i.e., the least negative value) is the least stable, occurring at the highest dopant concentration, 8.333 at. $\% \operatorname{Ti}\left(E_{b}=-6.94 \mathrm{eV}\right)$. As Ti concentration decreases, the $E_{b}$ decreases until it converges at 2.083 at. $\%$ Ti (-7.44 $\mathrm{eV})$, consistent with the structural convergence.

\section{Dopant Type}

To explore a wide range of dopant effects on 2D MoX 2 and 2D WX 2 systems, six different transition-metal-site dopants were considered: $\mathrm{Mo}, \mathrm{Ni}, \mathrm{Sc}, \mathrm{Ti}, \mathrm{V}$, and $\mathrm{W}$. The chemical formula for these compounds is $\mathrm{M}^{\prime} \mathrm{M}_{15} \mathrm{X}_{32}(\mathrm{M}=\mathrm{Mo}$ or $\mathrm{W}$; $\mathrm{X}=\mathrm{S}, \mathrm{Se}$, or Te). DFT-based structural relaxation calculations were employed to obtain ground state configurations for each doped system. Structural property results are given in Table 6.2. In most cases, the structural distortion is minimal for 2.083 at. $\%$ dopant on $2 \mathrm{D} \mathrm{MoX}_{2}$ and 2D WX 2 . For both structures, the Ni and Sc dopants cause the largest distortions (as 
much as $10.66 \%$ and $12.78 \%$ reductions in monolayer thickness (X-X bond lengths) for $\mathrm{Ni}$ in $\mathrm{MoTe}_{2}$ and $\mathrm{WTe}_{2}$, respectively). These two dopants have the lowest oxidation states of any metals investigated ( +2 for $\mathrm{Ni}$ and +3 for $\mathrm{Sc}$ ) and have respectively, the smallest and largest atomic radii. The tellurides consistently exhibit larger distortions than the other dichalcogenides. These distortions are most noticeable in the layer thickness, corresponding to $X-X$ bond length $\left(\mathrm{d}_{\mathrm{X}-\mathrm{X}}\right)$, and $\mathrm{X}-\mathrm{M}-\mathrm{X}$ bond angle $(\theta)$. The least distorted structures are $\mathrm{W}$-doped $\mathrm{MoX}_{2}$ and Mo-doped $\mathrm{WX}_{2}$. This is consistent with the fact that Mo and W have similar atomic radii $(0.79 \AA$ and $0.80 \AA$, respectively) and tend to have similar oxidation states (+6). Overall, the lattice constant, auc, experiences the least distortion of all measured geometries ( $\leq|1.00 \%|$ change). This implies that differences in layer thickness, rather than lattice mismatch, would be the primary cause for any potential dopant instability.

Table 6.2. Structure properties of metal-site doped $M X_{2}$ (having the formula $M^{\prime} M_{15} X_{32}$ where $M^{\prime}=$ dopant atom Mo, $\mathrm{Ni}, \mathrm{Sc}, \mathrm{Ti}, \mathrm{V}$, or $\mathrm{W}$; $\mathrm{M}=\mathrm{Mo}$ or $\mathrm{W}$; and $\mathrm{X}=$ $\mathrm{S}$, Se, or Te) compared to the undoped values. The average lattice parameter per unit cell ( $4 \times 4$ unit cells per structure), auc, $M^{\prime}-X$ bond length, $X-X$ bond length, and $X-M-X$ bond angle are given in units of either angstroms $(\AA)$ or degrees $\left({ }^{\circ}\right)$. The percent difference from the corresponding undoped value is listed as $\Delta \%$.

\begin{tabular}{|c|c|c|c|c|c|c|c|c|c|}
\hline \multirow{2}{*}{$\begin{array}{c}\mathrm{MX}_{2} \\
\text { matrix }\end{array}$} & $\begin{array}{c}\text { M, } \\
\text { dopant }\end{array}$ & \multicolumn{2}{|c|}{$\mathrm{a}_{\text {uc }}$ (avg./unitcell) } & \multicolumn{2}{|c|}{$\mathrm{d}_{\mathrm{M}-\mathrm{X}}$} & \multicolumn{2}{|c|}{$\mathrm{d}$ X-X } & \multicolumn{2}{|c|}{$\theta$} \\
\cline { 3 - 10 } & $\AA$ & $\Delta \%$ & $\AA$ & $\Delta \%$ & $\AA$ & $\Delta \%$ & $\circ$ & $\Delta \%$ \\
\hline \multirow{3}{*}{} & undoped & 3.182 & -- & 2.413 & -- & 3.128 & -- & 80.81 & -- \\
\cline { 2 - 11 } & $\mathrm{Ni}$ & 3.189 & $0.23 \%$ & 2.417 & $0.16 \%$ & 3.078 & $-1.60 \%$ & 79.10 & $-2.11 \%$ \\
\cline { 2 - 11 } $\mathrm{MoS}_{2}$ & $\mathrm{Sc}$ & 3.213 & $0.97 \%$ & 2.526 & $4.70 \%$ & 3.115 & $-0.40 \%$ & 76.13 & $-5.79 \%$ \\
\cline { 2 - 11 } & $\mathrm{Ti}$ & 3.203 & $0.66 \%$ & 2.428 & $0.63 \%$ & 3.056 & $-2.30 \%$ & 77.99 & $-3.48 \%$ \\
\cline { 2 - 10 } & $\mathrm{V}$ & 3.188 & $0.17 \%$ & 2.362 & $-2.11 \%$ & 3.043 & $-2.71 \%$ & 80.21 & $-0.74 \%$ \\
\cline { 2 - 10 } & $\mathrm{W}$ & 3.182 & $0.00 \%$ & 2.409 & $-0.14 \%$ & 3.128 & $0.00 \%$ & 80.94 & $0.17 \%$ \\
\hline
\end{tabular}




\begin{tabular}{|c|c|c|c|c|c|c|c|c|c|}
\hline \multirow{6}{*}{$\mathrm{MoSe}_{2}$} & undoped & 3.319 & -- & 2.541 & -- & 3.338 & -- & 82.12 & -- \\
\hline & $\mathrm{Ni}$ & 3.327 & $0.25 \%$ & 2.480 & $-2.41 \%$ & 3.190 & $-4.45 \%$ & 80.05 & $-2.52 \%$ \\
\hline & $\mathrm{Sc}$ & 3.341 & $0.68 \%$ & 2.649 & $4.25 \%$ & 3.375 & $1.11 \%$ & 79.15 & $-3.62 \%$ \\
\hline & $\mathrm{Ti}$ & 3.329 & $0.33 \%$ & 2.557 & $0.61 \%$ & 3.301 & $-1.11 \%$ & 80.42 & $-2.07 \%$ \\
\hline & V & 3.316 & $-0.08 \%$ & 2.492 & $-1.93 \%$ & 3.278 & $-1.79 \%$ & 82.27 & $0.18 \%$ \\
\hline & W & 3.318 & $-0.01 \%$ & 2.541 & $0.01 \%$ & 3.341 & $0.07 \%$ & 82.19 & $0.08 \%$ \\
\hline \multirow{6}{*}{$\mathrm{MoTe}_{2}$} & undoped & 3.549 & -- & 2.731 & -- & 3.611 & -- & 82.76 & -- \\
\hline & $\mathrm{Ni}$ & 3.564 & $0.40 \%$ & 2.595 & $-4.99 \%$ & 3.226 & $-10.66 \%$ & 76.87 & $-7.12 \%$ \\
\hline & $\mathrm{Sc}$ & 3.570 & $0.59 \%$ & 2.853 & $4.46 \%$ & 3.653 & $1.17 \%$ & 79.63 & $-3.79 \%$ \\
\hline & $\mathrm{Ti}$ & 3.556 & $0.18 \%$ & 2.757 & $0.94 \%$ & 3.599 & $-0.34 \%$ & 81.49 & $-1.53 \%$ \\
\hline & V & 3.549 & $-0.02 \%$ & 2.693 & $-1.41 \%$ & 3.547 & $-1.78 \%$ & 82.39 & $-0.45 \%$ \\
\hline & W & 3.550 & $0.01 \%$ & 2.734 & $0.12 \%$ & 3.614 & $0.08 \%$ & 82.72 & $-0.05 \%$ \\
\hline \multirow{6}{*}{$\mathrm{WS}_{2}$} & undoped & 3.181 & -- & 2.416 & -- & 3.139 & -- & 81.03 & -- \\
\hline & Mo & 3.182 & $0.02 \%$ & 2.420 & $0.14 \%$ & 3.138 & $-0.06 \%$ & 80.84 & $-0.24 \%$ \\
\hline & $\mathrm{Ni}$ & 3.191 & $0.30 \%$ & 2.419 & $0.13 \%$ & 3.075 & $-2.06 \%$ & 78.92 & $-2.61 \%$ \\
\hline & $\mathrm{Sc}$ & 3.213 & $1.00 \%$ & 2.525 & $4.49 \%$ & 3.114 & $-0.81 \%$ & 76.16 & $-6.02 \%$ \\
\hline & $\mathrm{Ti}$ & 3.202 & $0.64 \%$ & 2.432 & $0.65 \%$ & 3.066 & $-2.35 \%$ & 78.15 & $-3.56 \%$ \\
\hline & V & 3.186 & $0.15 \%$ & 2.369 & $-1.94 \%$ & 3.058 & $-2.60 \%$ & 80.37 & $-0.82 \%$ \\
\hline \multirow{6}{*}{$\mathrm{WSe}_{2}$} & undoped & 3.317 & -- & 2.546 & -- & 3.355 & -- & 82.43 & -- \\
\hline & Mo & 3.316 & $-0.01 \%$ & 2.545 & $-0.02 \%$ & 3.351 & $-0.13 \%$ & 82.33 & $-0.13 \%$ \\
\hline & $\mathrm{Ni}$ & 3.326 & $0.27 \%$ & 2.528 & $-0.68 \%$ & 3.258 & $-2.89 \%$ & 80.22 & $-2.69 \%$ \\
\hline & $\mathrm{Sc}$ & 3.348 & $0.94 \%$ & 2.649 & $4.04 \%$ & 3.326 & $-0.85 \%$ & 77.80 & $-5.62 \%$ \\
\hline & $\mathrm{Ti}$ & 3.329 & $0.36 \%$ & 2.559 & $0.51 \%$ & 3.304 & $-1.50 \%$ & 80.44 & $-2.42 \%$ \\
\hline & V & 3.315 & $-0.07 \%$ & 2.497 & $-1.93 \%$ & 3.290 & $-1.93 \%$ & 82.43 & $0.00 \%$ \\
\hline
\end{tabular}




\begin{tabular}{|c|c|c|c|c|c|c|c|c|c|}
\hline \multirow{5}{*}{$\mathrm{WTe}_{2}$} & undoped & 3.552 & -- & 2.736 & -- & 3.623 & -- & 82.91 & - \\
\cline { 2 - 10 } & $\mathrm{Mo}$ & 3.552 & $0.00 \%$ & 2.732 & $-0.13 \%$ & 3.618 & $-0.13 \%$ & 82.91 & $0.01 \%$ \\
\cline { 2 - 10 } & $\mathrm{Ni}$ & 3.566 & $0.39 \%$ & 2.592 & $-5.26 \%$ & 3.160 & $-12.78 \%$ & 75.10 & $-9.42 \%$ \\
\cline { 2 - 10 } & $\mathrm{Sc}$ & 3.578 & $0.74 \%$ & 2.848 & $4.08 \%$ & 3.608 & $-0.41 \%$ & 78.61 & $-5.18 \%$ \\
\cline { 2 - 10 } & $\mathrm{Ti}$ & 3.561 & $0.25 \%$ & 2.755 & $0.69 \%$ & 3.567 & $-1.53 \%$ & 80.69 & $-2.67 \%$ \\
\cline { 2 - 10 } & $\mathrm{V}$ & 3.551 & $-0.01 \%$ & 2.693 & $-1.58 \%$ & 3.541 & $-2.24 \%$ & 82.23 & $-0.82 \%$ \\
\hline
\end{tabular}

The resulting $E_{b}$ values for each of the doped 2D MoX 2 and 2D WX 2 systems, given in Figure 6.3, are depicted graphically versus the dopant atom. The dopant atoms are listed in order of increasing atomic radius. Here we can see that the Ni dopant has the least negative binding energy among all systems (ranging from $-1.60 \mathrm{eV}$ in $\mathrm{WTe}_{2}$ to -2.50 $\mathrm{eV}$ in $\mathrm{MoS}_{2}$ ), indicating that it is the least stable dopant observed. This could be due to a number of factors including: $\mathrm{Ni}$ is the only magnetic dopant investigated, it has the lowest average oxidation state $(+2)$, and it has the smallest atomic radius $(0.62 \AA)$. Alternatively, the Sc and Ti dopants consistently exhibit among the most negative binding energies, suggesting good stability in each system. These values range from -4.58 $\mathrm{eV}(\mathrm{Sc})$ and $-4.37 \mathrm{eV}(\mathrm{Ti})$ in both telluride systems to $-6.98 \mathrm{eV}(\mathrm{Sc})$ and $-7.44 \mathrm{eV}(\mathrm{Ti})$ in $\mathrm{MoS}_{2}$. This is in contrast to the fact that the Sc-doped systems have among the largest distortions, suggesting that atomic radius of the dopants plays a larger role in its stability than the oxidation state. All dopants in $\mathrm{MoS}_{2}$, except Ni, have large negative binding energies (around $-7 \mathrm{eV}$ ) suggesting high stability. However, the dopants show noticeably decreasing stability with increasing chalcogen atomic number. Also, the difference in binding energy between the $\mathrm{MoX}_{2}$ and $\mathrm{WX}_{2}$ systems is less than $1 \mathrm{eV}$. The largest difference is $0.86 \mathrm{eV}$, occurring between the $\mathrm{V}$-doped $\mathrm{MSe}_{2}$ systems. 


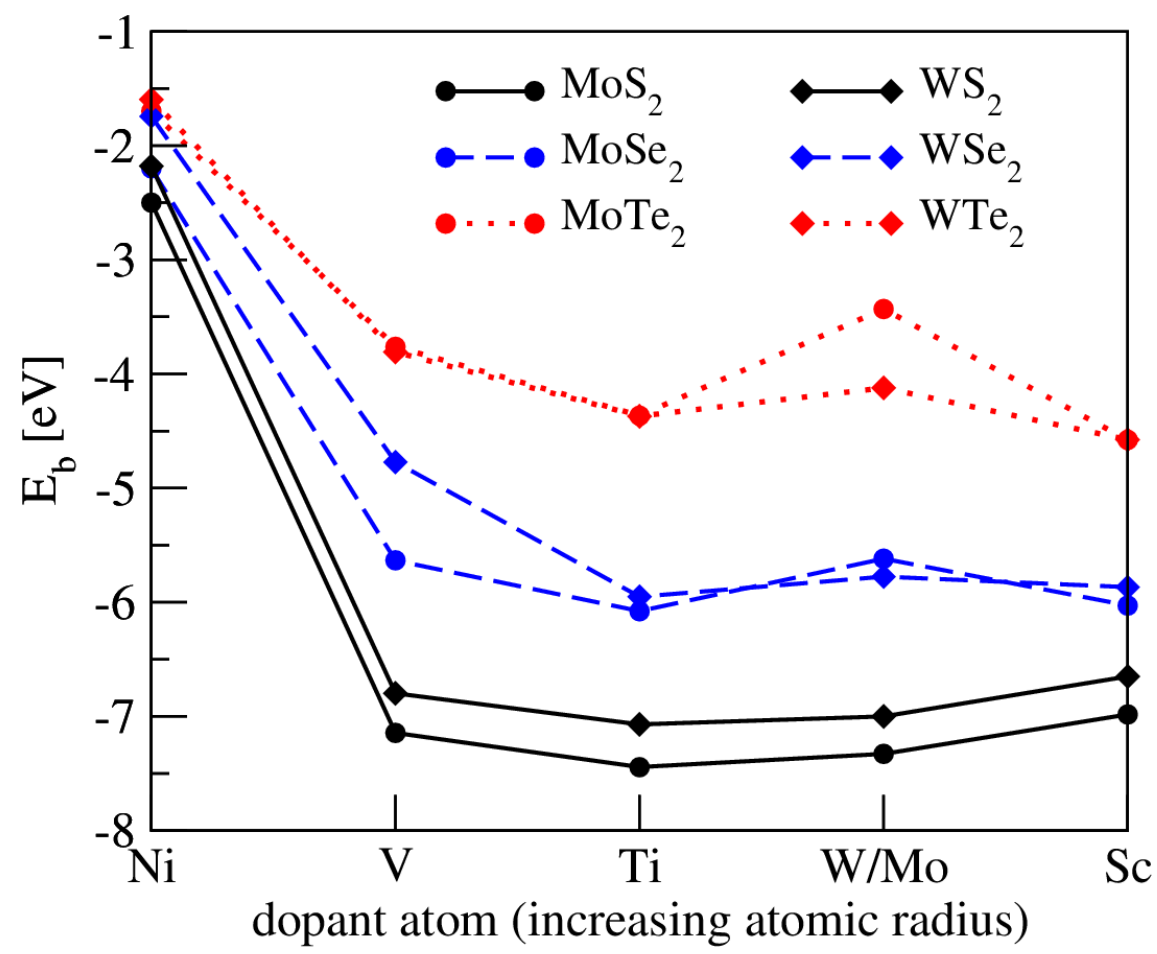

Figure 6.3. Dopant atom binding energy $\left(E_{b}\right)$ for each system listed in order of increasing dopant atomic radius. Solid black, dashed blue, and dotted red lines represent $\mathrm{MS}_{2}, \mathrm{MSe}_{2}$, and $\mathrm{MTe}_{2}$ systems, respectively. Circle-lines depict $\mathrm{MoX}_{2}$ structures while diamond-lines depict $\mathbf{W X}_{2}$ structures. More-negative $E_{b}$ values (greater absolute values) correspond to greater stability.

\section{Electrical Properties}

The incorporation of Ti dopants has a significant impact on the electrical properties of 2D MoS 2 . Figure 6.4 depicts the electronic density of states (DoS) for pure $\mathrm{MoS}_{2}$ (black line), plotted against the DoS for Ti-doped $\mathrm{MoS}_{2}$ (colored lines). In each case, Ti dopants decrease the conduction band minimum (CBM) - although not by much for the case with largest Ti concentration - and introduces a mid-gap peak just above the Fermi level $\left(E_{F}\right)$ making the system behave as a $p$-type semiconductor. The DoS plots also converge as Ti concentration decreases. Each of the three relatively low concentrations $(0.926,1.333$, and 2.083 at.\% Ti) have very similar results, implying that dopant concentrations at or below 2.083 at.\% Ti exhibit similar electronic behavior. This result is consistent with what we found in the structural results. The lowest concentration 
of Ti (0.926 at.\% Ti) has a negligible DoS at $E_{F}$, yet as Ti concentration increases, this value increases until 3.704 at.\% $\mathrm{Ti}$ when the mid-gap peak has essentially blended into the valence band. These results could suggest an increase in electrical conductivity for the Ti-doped system when compared to the pure. The system with the highest Ti concentration (8.333 at.\% Ti) deviates the most from the pure $2 \mathrm{D} \mathrm{MoS}_{2}$ data and has a valence band maximum (VBM) that extends well beyond $E_{F}$. With such a high concentration, the dopant-dopant separation is only about $6.47 \AA$ and the Ti is more of a constituent atom than a dopant.

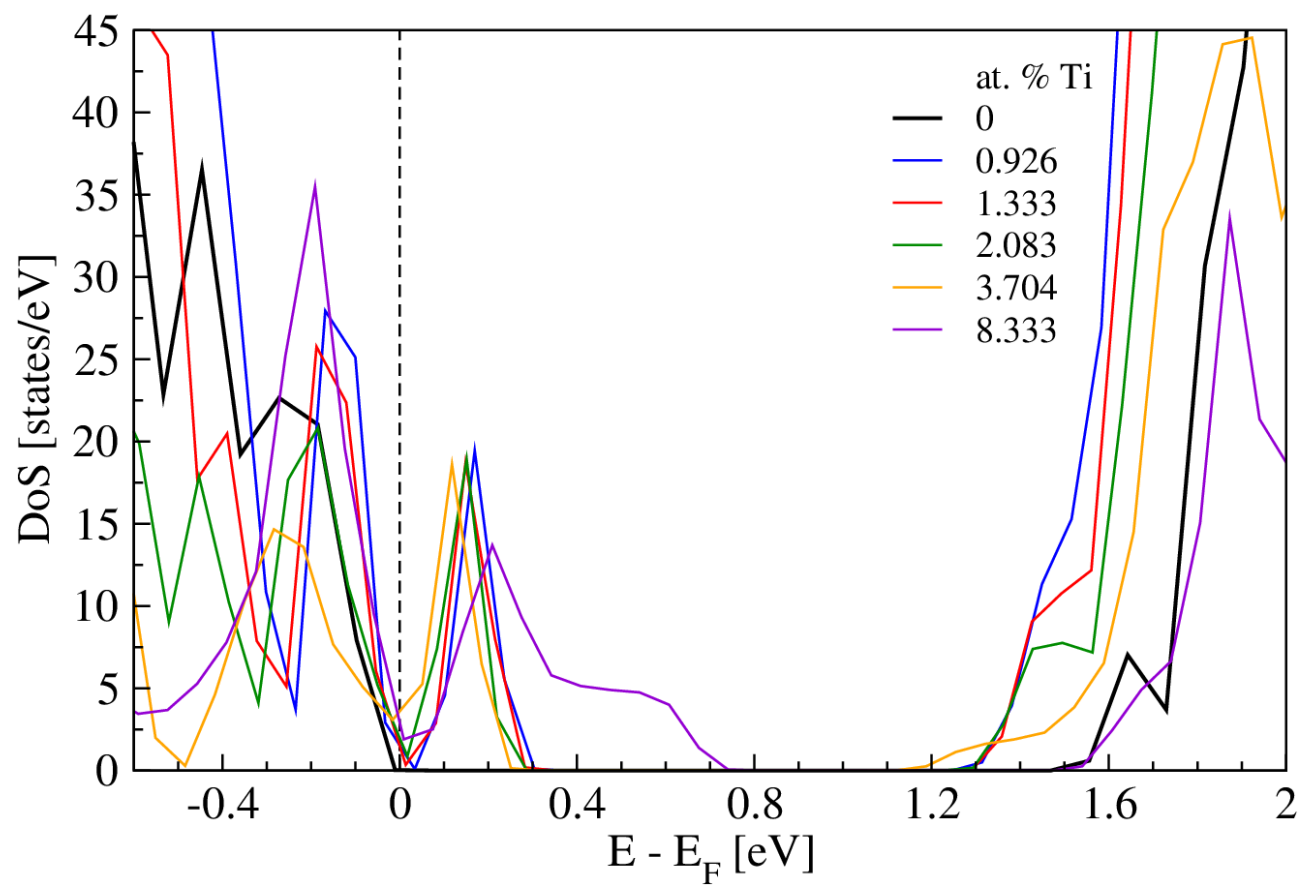

Figure 6.4. Electronic $\mathrm{DoS}$ for $2 \mathrm{D} \mathrm{MoS}_{2}$ having increasing atomic percentage concentrations of Ti dopants. These concentrations include 0.926 (blue), 1.333 (red), 2.083 (green), 3.704 (orange), and 8.333 (violet) at.\% Ti. The pure $\mathrm{MoS}_{2}$ data is shown for comparison and depicted with a black line. All data is shifted to align each plot's respective Fermi levels ( $E_{F}$ 's) to $x=0$ (represented by the vertical dashed black line). Inset focuses on the area immediately surrounding $E_{F}$. 
Similarly, the electronic structure for the V doped system was calculated for comparison. Figure 6.5 depicts the local density of states (LDoS) for the individual atomic species' contribution to the DoS. These were plotted against the DoS for pure $\mathrm{MoS}_{2}$ (black) for comparison. Both dopants have similar effects on the DoS such as a slight reduction in the CBM position and the addition of a mid-gap peak near the VBM. The V-doped system has more DoS near $E_{F}$, but the mid-gap peak is less substantial than that of the Ti-doped system. The LDoS results show that while this peak is primarily contributed by the dopant atom for both systems, there are still significant contributions from the Mo and $\mathrm{S}$ atoms. This implies a change in electronic structure for the overall system. These results indicate a $p$-type semiconducting behavior for both dopant types but the effect will likely be more prominent for Ti. 


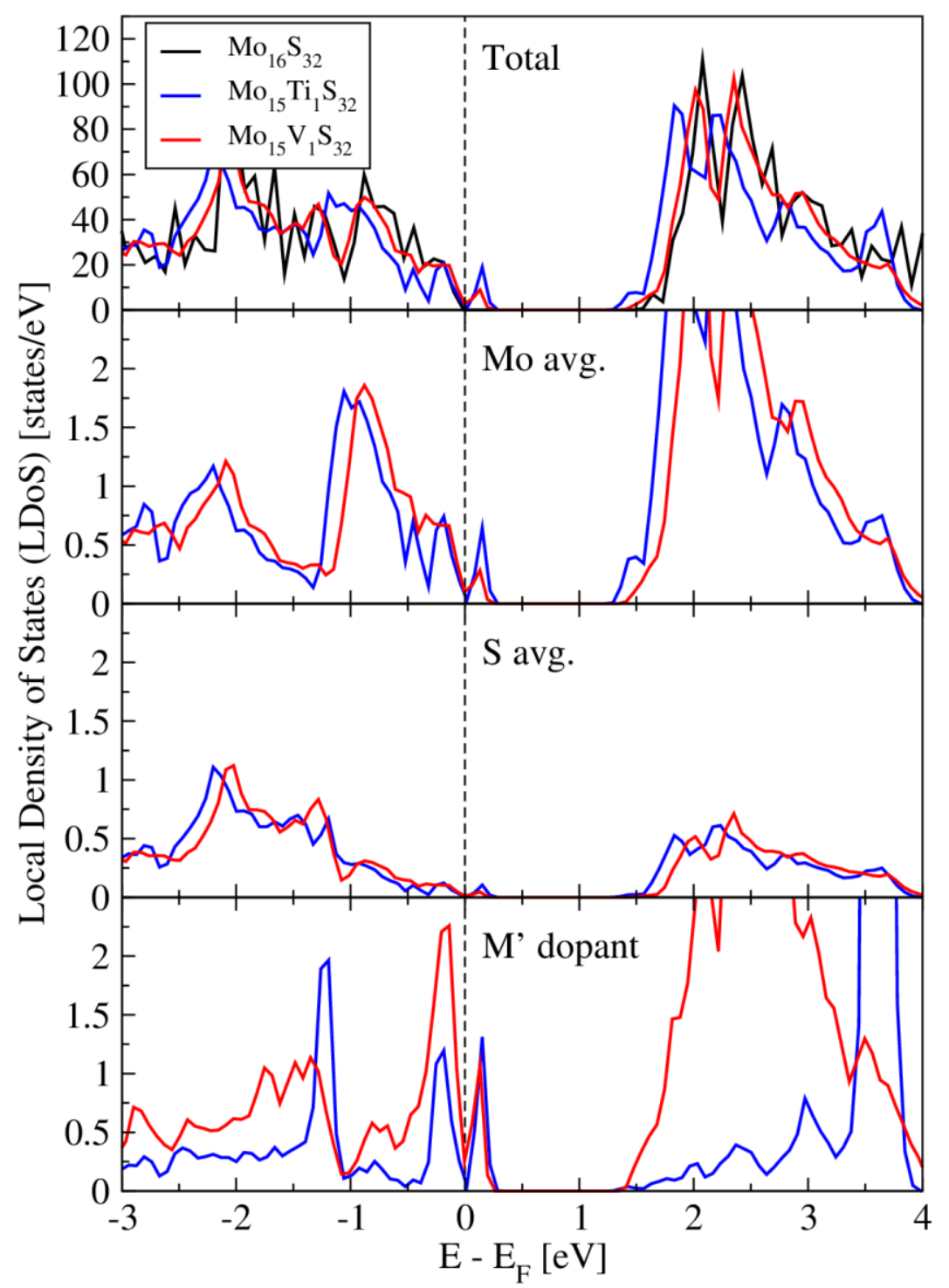

Figure 6.5. LDoS calculated as a function of electron energy ( $\operatorname{shifted}$ to $E_{F}=0 \mathrm{eV}$ ) for the Mo15M' ${ }_{1} S_{32}$ system (2.083 at.\% M' where M' = Ti or V). The black line represents pure $\mathrm{MoS}_{2}$ while the blue and red lines depict Ti-doped and $\mathrm{V}$-doped $\mathrm{MoS}_{2}$ systems, respectively. The top plot has the total DoS for each system while the bottom three show local contributions per atom (avg.) from each of the Mo, S, and M' atoms, respectively. The vertical dashed black line indicates $E_{F}$.

The electronic structures were calculated for each doped system and compared with that of the undoped systems. All undoped 2D MX 2 systems were found to be direct bandgap semiconductors with gaps of 1.69, 1.84, 1.09, 1.84, 1.57, and $1.07 \mathrm{eV}$ for $\mathrm{MoS}_{2}$, $\mathrm{MoSe}_{2}, \mathrm{MoTe}_{2}, \mathrm{WS}_{2}, \mathrm{WSe} 2$, and $\mathrm{WTe}_{2}$, respectively. In Figure 6.6, the electronic density of states (DoS) is plotted for each dopant in each system while the undoped DoS data is 
included for comparison. For both $\mathrm{MoX}_{2}$ and $\mathrm{WX}_{2}$ systems, the bandgaps generally decrease with increasing chalcogen atomic number. In all cases, the presence of the transition metal dopant reduces the effective bandgaps by some amount. For some doped $\mathrm{MoS}_{2}$ and all doped $\mathrm{MoSe}_{2}$, there is a reduction in the conduction band minimum (CBM) whereas the dopants in $\mathrm{WS}_{2}$ and $\mathrm{WSe}_{2}$ have CBMs at or above that of the undoped structures (see Figure 6.6a - Figure 6.6d). Both telluride structures have a wide range of CBM values (see Figure 6.6e and Figure 6.6f). Most dopants also introduce mid-gap states which would increase electrical conductivity. In each system, these states are most prevalent near the top of the valence band, suggesting $p$-type semiconducting behavior. The Ni- and Sc-doped systems have the most mid-gap states while the Ti- and V-doped systems typically only show small mid-gap peaks. All Mo- and W-doped systems exhibit direct bandgap semiconducting behavior. In fact, with the exception of Sc-doped $\mathrm{MoSe}_{2}$ (which has a small gap of about $0.14 \mathrm{eV}$ ), these were the only doped systems found to be semiconducting. 

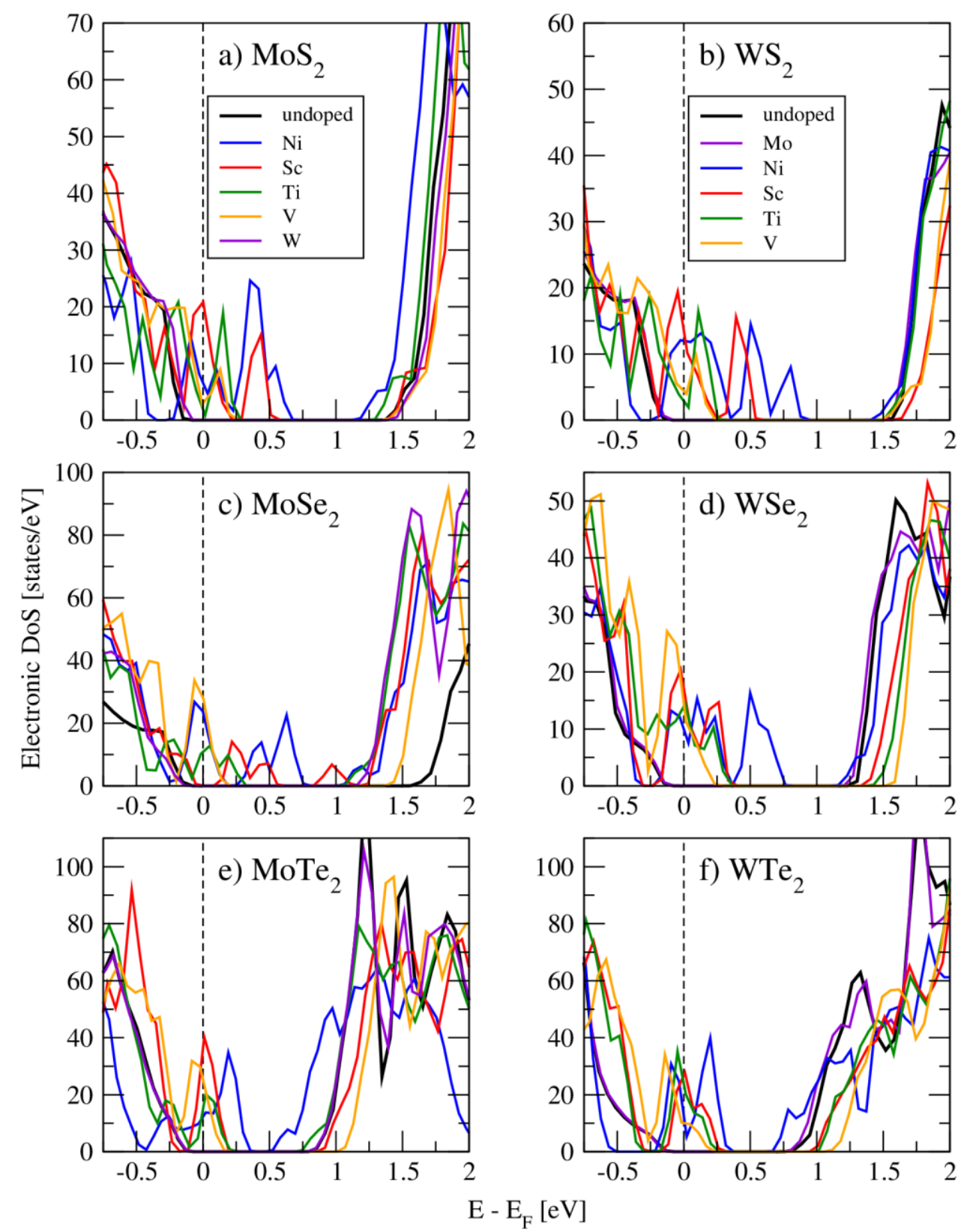

Figure 6.6. Electronic DoS for each doped system. Column 1 (a, c, and e) contains the $\mathrm{MoX}_{2}$ data while column 2 (b, d, and f) contains the $\mathrm{WX}_{2}$ data. The rows depict sulfide ( $a$ and $b$ ), selenide (c and $d$ ), and telluride (e and $f$ ) systems, respectively. Undoped, Ni-, Sc-, Ti-, and V-doped systems are represented by black, blue, red, green, and orange lines, respectively. Both the $\mathrm{W}$-doped systems in column 1 (a, c, and $e$ ) and the Mo-doped systems in column 2 (b, d, and f) are shown as violet lines.

\section{Electrical Transport Properties}

The semi-classic transport properties were calculated by solving the Boltzmann transport equation. ${ }^{45}$ The resulting $p$-type $S$, electrical conductivity, $\sigma$, and power factor 
$S^{2} \sigma$ are plotted in Figure 6.7. With the electronic density of states suggesting $p$-type behavior, we focused these calculations on $p$-type properties as holes are likely the majority carrier for these doped materials. For the sake of brevity, we focused on the only doped systems to have a sizeable bandgap with no mid-gap peaks which are W-doped $\mathrm{MoX}_{2}$ and the Mo-doped $\mathrm{WX}_{2}$. The difference in $S$ between Mo (Figure 6.7a) and W (Figure 6.7b) systems is small, thus they have comparable $S^{2} \sigma$ values (Figure 6.7e and Figure 6.7f). The largest difference between these two systems comes from their $\sigma$ values which were found to be larger for Mo-doped $\mathrm{WX}_{2}$ (Figure 6.7d) than for W-doped MoX 2 (Figure 6.7c). In all cases, $S^{2} \sigma$ is significantly reduced upon doping. This is largely due to $S$ which is reduced by as much as $15 \%$ for the $\mathrm{MoX}_{2}$ system (Figure $6.7 \mathrm{a}$ ) and $24 \%$ for the $\mathrm{WX}_{2}$ system (Figure 6.7b). In the case of the sulfides, the $\sigma$ is also reduced which leads to further reductions in $S$. For the selenides and tellurides, however, the $\sigma$ of the doped systems increases (by as much as $60 \%$ for the Mo-doped $\mathrm{WSe}_{2}$ compound at carrier concentration, $N=10^{14}$ holes $/ \mathrm{cm}^{2}$ ) which offsets the reduction in $S$. Therefore, the selenides and tellurides experience less of a reduction in $S^{2} \sigma$ upon doping than the sulfides for these systems. The systems that experience the least reduction are W-doped $\mathrm{MoSe}_{2}$ and $\mathrm{MoTe}_{2}$ which have $S^{2} \sigma$ values only $13 \%$ and $10 \%$ lower than undoped, respectively. 

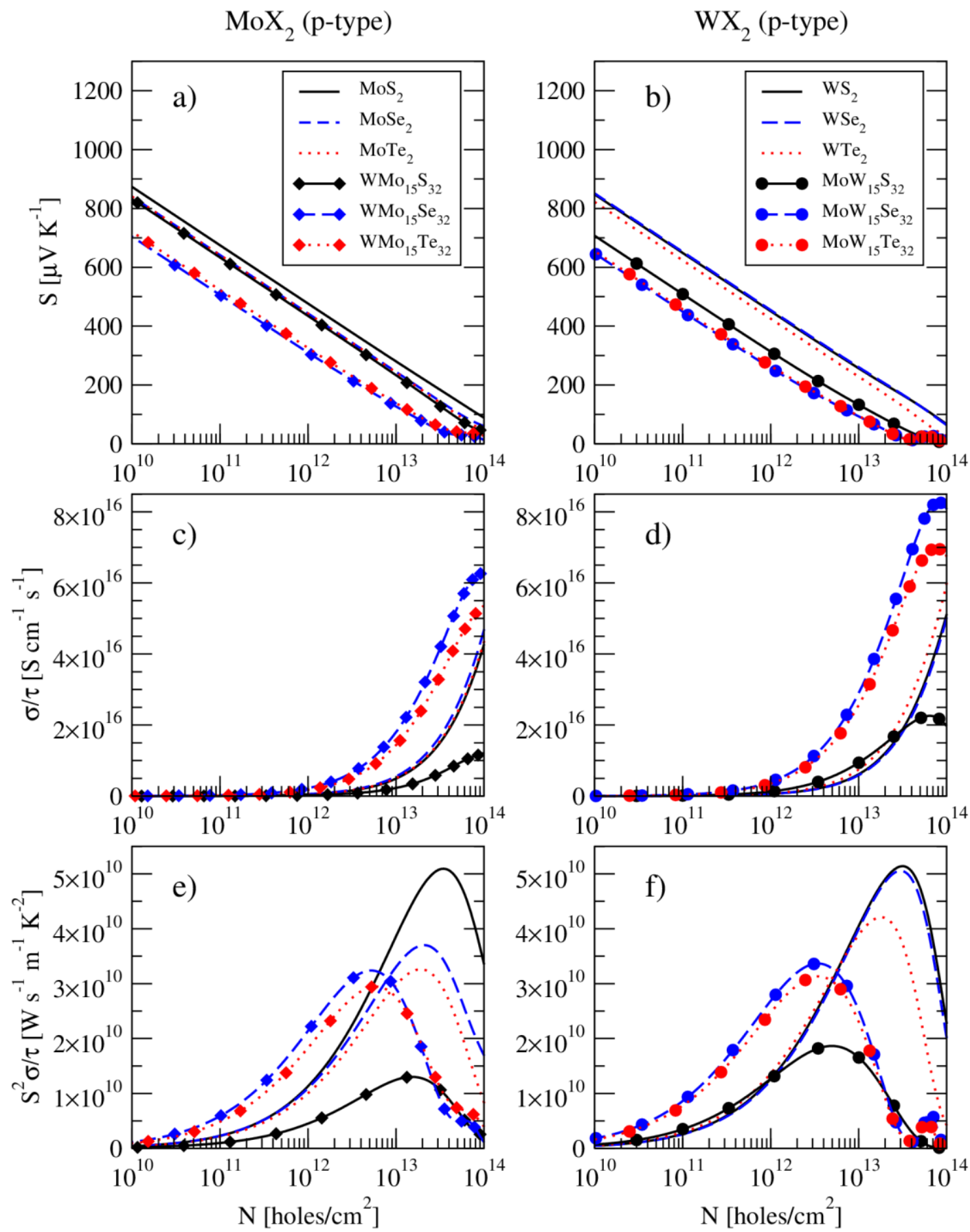

Figure 6.7. Boltzmann transport properties calculated as a function of carrier concentration, $N$, for W-doped $\mathrm{MoX}_{2}$ (plots a, c, and e) and Mo-doped WX2 (plots b, $d$, and f). The Seebeck coefficient, $S$, electrical conductivity, $\sigma / \tau$, and power factor, $S^{2} \sigma / \tau$, are shown in rows (a-b), (c-d), and (e-f), respectively. Sulfide, selenide, and telluride systems are represented by solid black, dashed blue, and dotted red plots, respectively, while diamond-line plots and circle-line plots depict $\mathrm{W}$ - and Mo-doped systems, respectively. 


\section{$\underline{\text { Phonon Properties }}$}

In semiconductors, heat conduction is typically dominated by phonon transport.

Therefore, it is necessary to investigate the effects of dopants on the phonon properties of 2D $\mathrm{MoS}_{2}$. The phonon DoS was calculated for each of the Ti-substituted 2D MoS 2

systems and plotted in Figure 6.8. Like the geometric and electronic structure results, the general shape, peak positions, and frequency gaps for the three lowest Ti concentrations are similar. Each of these has a mid-gap peak, effectively reducing the gap between acoustic and optical bands. The phonon DoS for the relatively high concentrations of Ti (3.704 and 8.333 at.\% Ti) start to deviate from that of the other systems. The reason for that is the significant structural distortion where the dopants are not adequately isolated. Similar to the electronic DoS results, the system with 8.333 at.\% Ti deviates the most and even has a larger frequency gap than the pure $\mathrm{MoS}_{2}$.

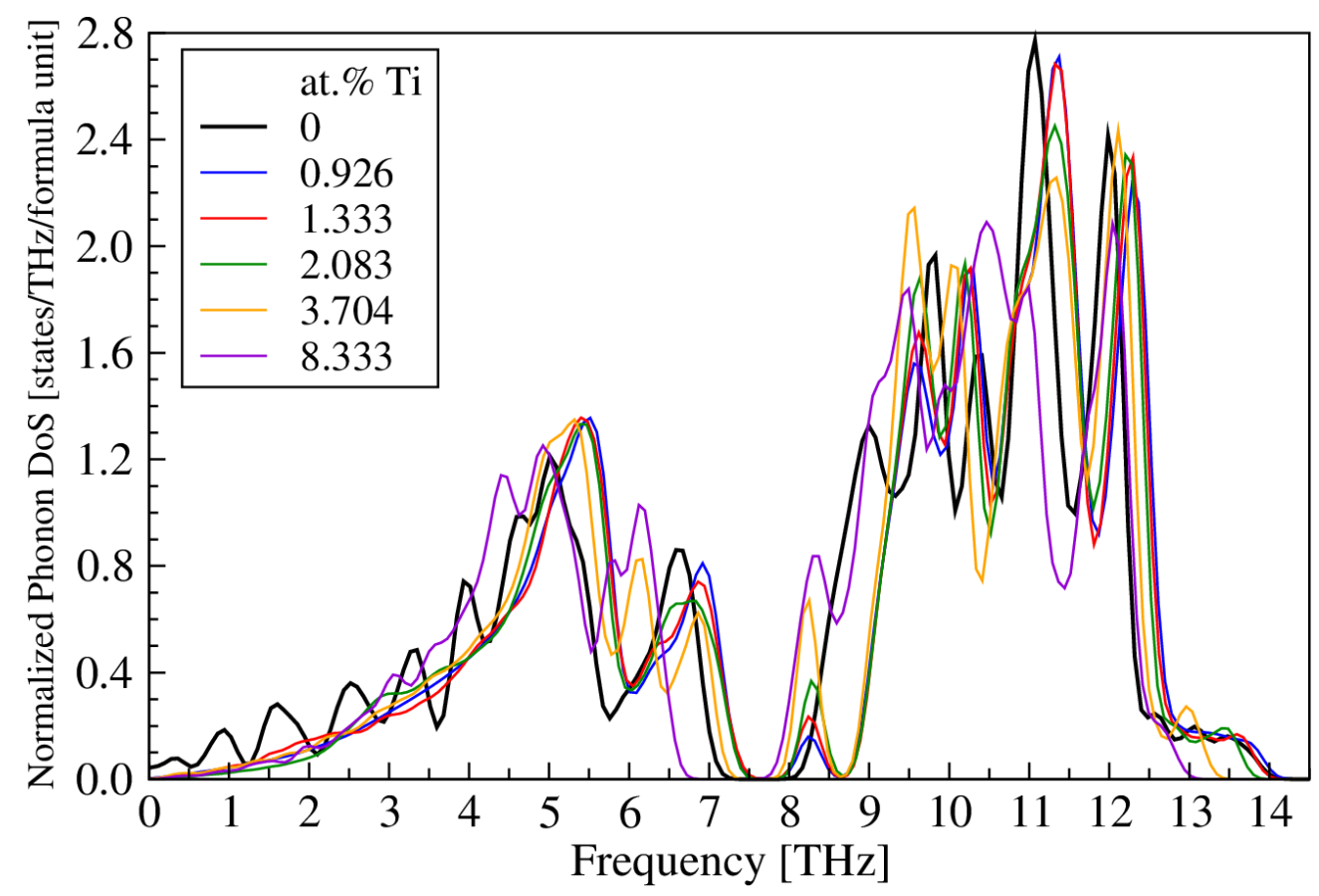

Figure 6.8. Phonon DoS calculated for various concentrations of Ti dopants in 2D MoS2. Data is shown normalized per formula unit. The black line represents pure 


\section{$\mathrm{MoS}_{2}$ with 0 at. \% Ti while the colored lines represent 0.926 (blue), 1.333 (red), 2.083 (green), 3.704 (orange), and 8.333 (violet) at. \% Ti.}

To compare the phonon properties of doped and undoped $\mathrm{MoS}_{2}$, the corresponding phonon spectra are qualitatively analyzed. This approach allows the effects of these dopants to be screened without necessitating rigorous Boltzmann transport equation (BTE) calculations. The results in Figure 6.8 show three primary alterations to the $\mathrm{MoS}_{2}$ phonon DoS upon Ti-doping: smoother features in the acoustic region, a reduced frequency gap, and a reduction in optical phonon mode frequency. For all concentrations of $\mathrm{Ti}$, the acoustic region of the phonon DoS is smoother than that of the pure $\mathrm{MoS}_{2}$ which has several peaks and deep valley-shaped curves. These peaks broaden and fill in valleys upon Ti-doping. In the work of Zhang et al., they suggested that phonon mode broadening indicated a reduction in phonon lifetimes and subsequently shorter mean free paths for modes in this region. ${ }^{48}$ The mid-gap peak causes the gap between acoustic and optical bands to decrease from $1.45 \mathrm{THz}$ for pure $\mathrm{MoS}_{2}$ down to 1.11 THz for 2.083 at.\% Ti. Previous first-principles calculations showed a correlation between smaller frequency gaps and reduced lattice thermal conductivity, $\kappa_{l}$, which is attributed to an increase in both the number and strength of scattering events between acoustic and optical phonon modes. ${ }^{11}$ Therefore, it is reasonable to assume that the presence of substitutional Ti here is likewise increasing acoustic-optical phonon scattering. Finally, in the optical band, the highest frequency modes decrease with increasing Ti concentration from about $14.3 \mathrm{THz}$ (at $0,0.926$, and 1.333 at.\% $\mathrm{Ti}$ ) down to about $13.1 \mathrm{THz}$ (at 8.333 at.\% Ti). This implies a lower dispersion gradient and therefore lower phonon group velocities for these modes. These alterations to the phonon 
properties of $2 \mathrm{D} \mathrm{MoS} 2$ indicate potential alterations to phonon transport in the material due to Ti doping.

The phonon DoS was also calculated for the V-doped system in comparison with the Ti-doped system (see Figure 6.9). The acoustic band region is smooth for both substitutions, implying an increase in phonon-phonon interaction. However, the optical band is not shifted to the lower frequencies as in the Ti case. The most significant difference between the Ti- and V-doped phonon DoS results is in the mid-gap peak which is shifted to higher frequencies for the V case. This shift causes the V-doped system to have effectively the same frequency gap $(1.43 \mathrm{THz})$ as that of the pure $\mathrm{MoS}_{2}(1.45 \mathrm{THz})$. Therefore, it might be expected that $\mathrm{V}$ dopants do not increase acoustic-optical phonon scattering in $2 \mathrm{D} \mathrm{MoS}$ as greatly as the Ti dopants. However, the bond lengths about the V dopant differ from that of both the Ti dopant and the Mo in the matrix. The work of Yildirim et al. showed a correlation between differing bond lengths and increased anharmonicity of phonon modes. ${ }^{49}$ This suggested that the V has greater anharmonicity than Ti. The V dopant also has a weaker binding energy than Ti (-7.15 eV compared to $7.44 \mathrm{eV}$ ) implying a lower sound velocity. For these reasons, we expect the nature of phonon transport in these two systems to differ. 


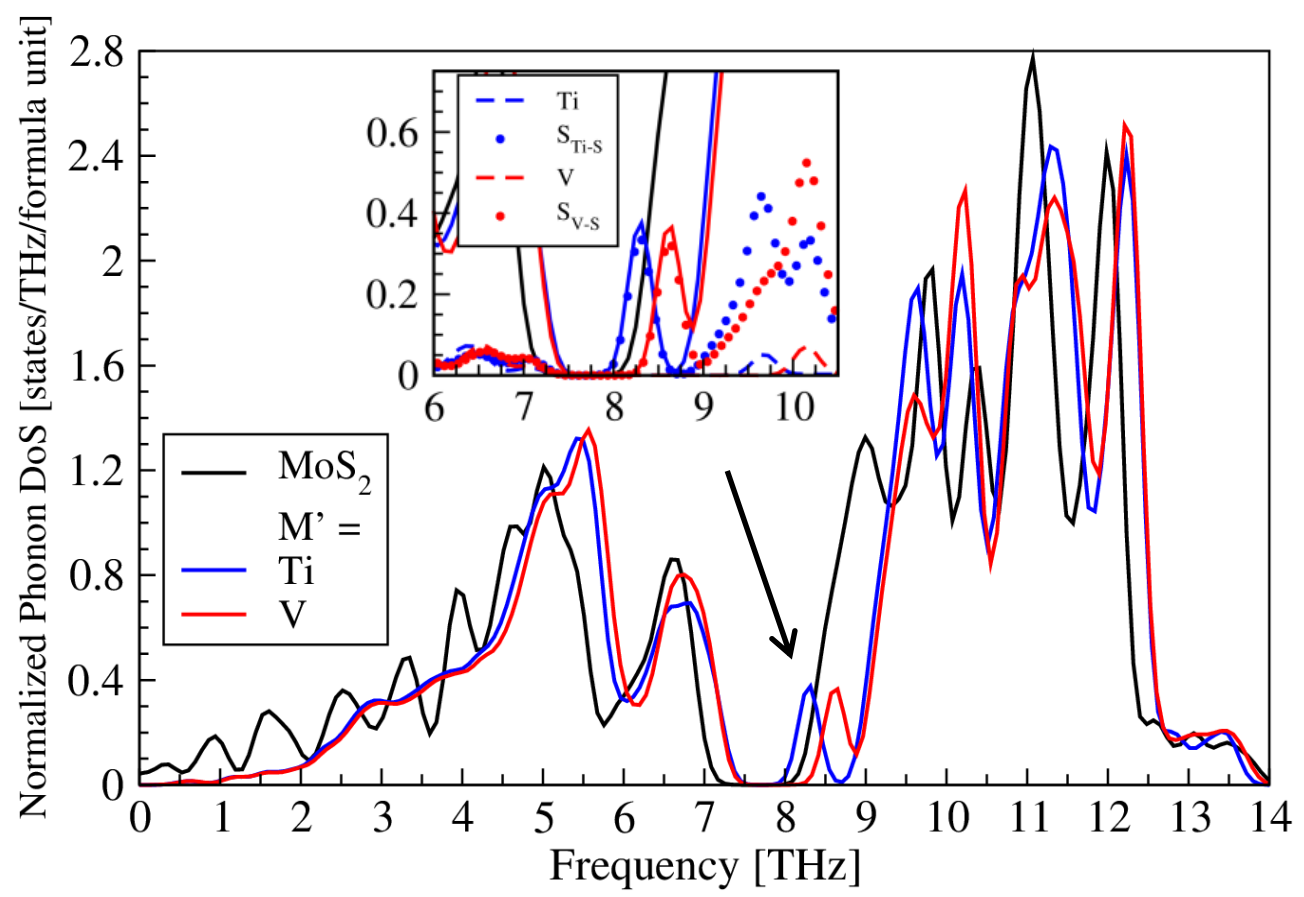

Figure 6.9. Total phonon DoS calculated for $\mathrm{MoS}_{2}$ doped with 2.083 at. \% Ti (blue) and 2.083 at.\% $\mathrm{V}$ (red). The black plot represents pure MoS2. Data is shown normalized per formula unit. Insert compares these results against the partial contributions from the dopant atoms (dashed lines) and $S$ atoms bonded to the dopants (circles), focusing on the frequency gap region.

Interestingly, the source of the mid-gap state is actually not the $\mathrm{V}$ and $\mathrm{Ti}$ atoms but instead the $\mathrm{S}$ atoms immediately neighboring the dopant site. The insert in Figure 6.9 shows the partial phonon DoS contributions from the dopant atoms individually as well as the $6 \mathrm{~S}$ atoms coordinated about the dopant. In both cases, the mid-gap peak is predominantly composed of those neighboring $\mathrm{S}$ atoms. This indicates that the nature of the M'-S bond differs from that of the Mo-S bond. Each of these results reveals significant alterations to the phonon properties of $2 \mathrm{D} \mathrm{MoS} 2$ upon Ti-/V-doping. With the potential increase in phonon mode scattering and reduction in phonon lifetimes, one could infer a corresponding reduction in $\kappa_{l}$. 


\subsection{Conclusion}

DFT-based calculations were conducted to investigate the effects of metal-site substitutional dopants on structural, energetic, electrical, transport, and phonon properties of $2 \mathrm{D} \mathrm{MX}$. The supercell approach was employed to simulate various concentrations of Ti dopants on $\mathrm{MoS}_{2}$. We found that increasing dopant concentration (8.333 at.\% Ti) does not greatly distort the $2 \mathrm{D} \mathrm{MoS}$ structure. The defects were found to be effectively isolated at a concentration of 2.083 at.\% Ti when their separation was about $12.8 \AA$. The effect of various dopants were screened at this concentration having the chemical formula $\mathrm{M}^{\prime} \mathrm{M}_{15} \mathrm{X}_{32}\left(\mathrm{M}^{\prime}=\mathrm{Mo}, \mathrm{Ni}, \mathrm{Sc}, \mathrm{Ti}, \mathrm{V}\right.$, or $\mathrm{W} ; \mathrm{M}=\mathrm{Mo}$ or $\mathrm{W}$; and $\mathrm{X}=\mathrm{S}$, Se, or Te). The dopants mostly exhibit minimal structural distortion on $2 \mathrm{D} \mathrm{MX}_{2}$ with the exception of $\mathrm{Ni}$ and Sc. Comparison of their geometry effects and binding energies suggests that their atomic radii may have a larger impact on dopant stability than their oxidation states. Binding energy results also indicate decreasing overall dopant stability with increasing chalcogen atomic number. Electronic bandgaps generally decrease with increasing $\mathrm{Ti}$ concentration, suggesting an increase in electrical conductivity. The general shape of the electronic DoS and phonon DoS results converge with decreasing Ti concentration until 2.083 at.\% Ti. Only the $\mathrm{W}$-doped $\mathrm{MoX}_{2}$ and Mo-doped $\mathrm{WX}_{2}$ are direct bandgap semiconductors. All other doped systems (with the exception of Sc-doped $\mathrm{MoSe}_{2}$ which has a very small bandgap) introduce mid-gap states on and above $E_{F}$ implying $p$-type semiconducting behavior and an increased electrical conductivity. This goes against the doping propensity of $\mathrm{MoS}_{2}$ which is experimentally determined to be $n$-type. Even the semiconducting Mo- and W-doped systems mostly have reduced bandgap sizes compared to the undoped systems. The $p$-type Boltzmann transport properties reveal a significant 
reduction in $S^{2} \sigma$ upon doping, despite many of these structures having small increases in $\sigma$. This is due to their reduced $S$ values. Features of the phonon DoS, e.g., acoustic region peak-broadening and narrower frequency gaps, indicate that Ti dopants cause more of a reduction in the lattice thermal conductivity of $2 \mathrm{D} \mathrm{MoS}_{2}$ than the $\mathrm{V}$ dopants. However, the shorter bond lengths about the V dopant and weaker covalent bonds suggest greater phonon anharmonicity, reducing the lattice thermal conductivity. This work explores various dopants and their effects on $2 \mathrm{D} \mathrm{MX}_{2}$ structures which is useful for guiding development of these materials for electronic and energy applications.

\section{Acknowledgements}

Computing facilities were provided by Boise State University's R1 cluster and the Idaho National Laboratory's high performance computing (HPC) center. The authors would like to thank the Research Experience for Undergraduates (REU) program at Boise State University as well as Ken Blair, Tyler Bevan, and Cormac Garvey for technical support. 


\section{References}

(1) Shishidou, T.; Freeman, A. J.; Asahi, R. Effect of GGA on the halfmetallicity of the itinerant ferromagnet CoS2. Phys Rev B 2001, 64, 180401.

(2) Ogawa, S. Magnetic-Properties of 3d Transition-Metal Dichalcogenides with the Pyrite Structure. J Appl Phys 1979, 50, 2308.

(3) Ataca, C.; Sahin, H.; Ciraci, S. Stable, Single-Layer MX2 TransitionMetal Oxides and Dichalcogenides in a Honeycomb-Like Structure. Journal of Physical Chemistry C 2012, 116, 8983.

(4) Rasmussen, F. A.; Thygesen, K. S. Computational 2D Materials Database: Electronic Structure of Transition-Metal Dichalcogenides and Oxides. Journal of Physical Chemistry C 2015, 119, 13169.

(5) Butler, S. Z.; Hollen, S. M.; Cao, L. Y.; Cui, Y.; Gupta, J. A.; Gutierrez, H. R.; Heinz, T. F.; Hong, S. S.; Huang, J. X.; Ismach, A. F.; Johnston-Halperin, E.; Kuno, M.; Plashnitsa, V. V.; Robinson, R. D.; Ruoff, R. S.; Salahuddin, S.; Shan, J.; Shi, L.; Spencer, M. G.; Terrones, M.; Windl, W.; Goldberger, J. E. Progress, Challenges, and Opportunities in Two-Dimensional Materials Beyond Graphene. Acs Nano 2013, 7, 2898.

(6) Gong, C.; Colombo, L.; Wallace, R. M.; Cho, K. The Unusual Mechanism of Partial Fermi Level Pinning at Metal-MoS2 Interfaces. Nano Lett 2014, 14, 1714.

(7) Wang, Q. H.; Kalantar-Zadeh, K.; Kis, A.; Coleman, J. N.; Strano, M. S. Electronics and optoelectronics of two-dimensional transition metal dichalcogenides. Nat Nanotechnol 2012, 7, 699.

(8) Huang, W.; Da, H. X.; Liang, G. C. Thermoelectric performance of MX2 (M = Mo, W; X= S, Se) monolayers. J Appl Phys 2013, 113, 104304.

(9) Huang, W.; Luo, X.; Gan, C. K.; Quek, S. Y.; Liang, G. C. Theoretical study of thermoelectric properties of few-layer MoS2 and WSe2. Physical Chemistry Chemical Physics 2014, 16, 10866. 
(10) Lee, C.; Hong, J.; Whangbo, M. H.; Shim, J. H. Enhancing the Thermoelectric Properties of Layered Transition-Metal Dichalcogenides 2HMQ(2) (M = Mo, W; Q = S, Se, Te) by Layer Mixing: Density Functional Investigation. Chem Mater 2013, 25, 3745.

(11) Gu, X. K.; Yang, R. G. Phonon transport in single-layer transition metal dichalcogenides: A first-principles study. Appl Phys Lett 2014, 105, 131903.

(12) Ganatra, R.; Zhang, Q. Few-Layer MoS2: A Promising Layered Semiconductor. Acs Nano 2014, 8, 4074.

(13) Lopez-Sanchez, O.; Lembke, D.; Kayci, M.; Radenovic, A.; Kis, A.

Ultrasensitive photodetectors based on monolayer MoS2. Nat Nanotechnol 2013, $8,497$.

(14) Baugher, B. W. H.; Churchill, H. O. H.; Yang, Y. F.; Jarillo-Herrero, P. Optoelectronic devices based on electrically tunable $\mathrm{p}$-n diodes in a monolayer dichalcogenide. Nat Nanotechnol 2014, 9, 262.

(15) Coleman, J. N.; Lotya, M.; O'Neill, A.; Bergin, S. D.; King, P. J.; Khan, U.; Young, K.; Gaucher, A.; De, S.; Smith, R. J.; Shvets, I. V.; Arora, S. K.; Stanton, G.; Kim, H. Y.; Lee, K.; Kim, G. T.; Duesberg, G. S.; Hallam, T.; Boland, J. J.; Wang, J. J.; Donegan, J. F.; Grunlan, J. C.; Moriarty, G.; Shmeliov, A.; Nicholls, R. J.; Perkins, J. M.; Grieveson, E. M.; Theuwissen, K.; McComb, D. W.; Nellist, P. D.; Nicolosi, V. Two-Dimensional Nanosheets Produced by Liquid Exfoliation of Layered Materials. Science 2011, 331, 568.

(16) Kam, K. K.; Parkinson, B. A. Detailed Photocurrent Spectroscopy of the Semiconducting Group-Vi Transition-Metal Dichalcogenides. J Phys Chem-Us 1982, $86,463$.

(17) Mak, K. F.; Lee, C.; Hone, J.; Shan, J.; Heinz, T. F. Atomically Thin MoS2: A New Direct-Gap Semiconductor. Phys Rev Lett 2010, 105, 136805.

(18) Kuc, A.; Zibouche, N.; Heine, T. Influence of quantum confinement on the electronic structure of the transition metal sulfide TS2. Phys Rev B 2011, 83, 245213. 
(19) Splendiani, A.; Sun, L.; Zhang, Y. B.; Li, T. S.; Kim, J.; Chim, C. Y.; Galli, G.; Wang, F. Emerging Photoluminescence in Monolayer MoS2. Nano Lett 2010, 10, 1271.

(20) Yin, Z. Y.; Li, H.; Li, H.; Jiang, L.; Shi, Y. M.; Sun, Y. H.; Lu, G.; Zhang, Q.; Chen, X. D.; Zhang, H. Single-Layer MoS2 Phototransistors. Acs Nano 2012, 6,74 .

(21) Hicks, L. D.; Dresselhaus, M. S. Effect of Quantum-Well Structures on the Thermoelectric Figure of Merit. Phys Rev B 1993, 47, 12727.

(22) Hicks, L. D.; Dresselhaus, M. S. Thermoelectric Figure of Merit of a OneDimensional Conductor. Phys Rev B 1993, 47, 16631.

(23) Mahan, G. D.; Sofo, J. O. The best thermoelectric. P Natl Acad Sci USA 1996, 93, 7436 .

(24) Zahid, F.; Lake, R. Thermoelectric properties of Bi2Te3 atomic quintuple thin films. Appl Phys Lett 2010, 97, 212102.

(25) Wickramaratne, D.; Zahid, F.; Lake, R. K. Electronic and thermoelectric properties of few-layer transition metal dichalcogenides. J Chem Phys 2014, 140, 124710.

(26) Hochbaum, A. I.; Chen, R. K.; Delgado, R. D.; Liang, W. J.; Garnett, E. C.; Najarian, M.; Majumdar, A.; Yang, P. D. Enhanced thermoelectric performance of rough silicon nanowires. Nature 2008, 451, 163.

(27) Boukai, A. I.; Bunimovich, Y.; Tahir-Kheli, J.; Yu, J. K.; Goddard, W. A.; Heath, J. R. Silicon nanowires as efficient thermoelectric materials. Nature 2008, $451,168$.

(28) Williamson, I.; Correa Hernandez, A.; Wong-Ng, W.; Li, L. HighThroughput Computational Screening of Electrical and Phonon Properties of Two-Dimensional Transition Metal Dichalcogenides. JOM 2016, 68, 2666. (29) Graedel, T. E.; Harper, E. M.; Nassar, N. T.; Nuss, P.; Reck, B. K. Criticality of metals and metalloids. P Natl Acad Sci USA 2015, 112, 4257. 
(30) Zhou, Y. G.; Su, Q. L.; Wang, Z. G.; Deng, H. Q.; Zu, X. T. Controlling magnetism of MoS2 sheets by embedding transition-metal atoms and applying strain. Physical Chemistry Chemical Physics 2013, 15, 18464.

(31) Cheng, Y. C.; Zhu, Z. Y.; Mi, W. B.; Guo, Z. B.; Schwingenschlogl, U. Prediction of two-dimensional diluted magnetic semiconductors: Doped monolayer MoS2 systems. Phys Rev B 2013, 87, 100401.

(32) Ramasubramaniam, A.; Naveh, D. Mn-doped monolayer MoS2: An atomically thin dilute magnetic semiconductor. Phys Rev B 2013, 87, 195201.

(33) Saab, M.; Raybaud, P. Tuning the Magnetic Properties of MoS2 Single Nanolayers by 3d Metals Edge Doping. Journal of Physical Chemistry C 2016, $120,10691$.

(34) Chen, Y. F.; Xi, J. Y.; Dumcenco, D. O.; Liu, Z.; Suenaga, K.; Wang, D.; Shuai, Z. G.; Huang, Y. S.; Xie, L. M. Tunable Band Gap Photoluminescence from Atomically Thin Transition-Metal Dichalcogenide Alloys. Acs Nano 2013, 7, 4610 .

(35) Suh, J.; Park, T. E.; Lin, D. Y.; Fu, D. Y.; Park, J.; Jung, H. J.; Chen, Y. B.; Ko, C.; Jang, C.; Sun, Y. H.; Sinclair, R.; Chang, J.; Tongay, S.; Wu, J. Q. Doping against the Native Propensity of MoS2: Degenerate Hole Doping by Cation Substitution. Nano Lett 2014, 14, 6976.

(36) Hsu, W. K.; Zhu, Y. Q.; Yao, N.; Firth, S.; Clark, R. J. H.; Kroto, H. W.; Walton, D. R. M. Titanium-doped molybdenum disulfide nanostructures. Advanced Functional Materials 2001, 11, 69.

(37) Deepak, F. L.; Cohen, H.; Cohen, S.; Feldman, Y.; Popovitz-Biro, R.; Azulay, D.; Millo, O.; Tenne, R. Fullerene-like (IF) NbxMo1-xS2 nanoparticles. J Am Chem Soc 2007, 129, 12549.

(38) Sun, Q. C.; Yadgarov, L.; Rosentsveig, R.; Seifert, G.; Tenne, R.; Musfeldt, J. L. Observation of a Burstein-Moss Shift in Rhenium-Doped MoS2 Nanoparticles. Acs Nano 2013, 7, 3506. 
(39) Yadgarov, L.; Rosentsveig, R.; Leitus, G.; Albu-Yaron, A.; Moshkovich, A.; Perfilyev, V.; Vasic, R.; Frenkel, A. I.; Enyashin, A. N.; Seifert, G.; Rapoport, L.; Tenne, R. Controlled Doping of MS2 (M=W, Mo) Nanotubes and Fullerenelike Nanoparticles. Angew Chem Int Edit 2012, 51, 1148.

(40) Kresse, G.; Furthmuller, J. Efficient iterative schemes for ab initio totalenergy calculations using a plane-wave basis set. Phys Rev B 1996, 54, 11169.

(41) Perdew, J. P.; Burke, K.; Ernzerhof, M. Generalized gradient approximation made simple. Phys Rev Lett 1996, 77, 3865.

(42) Blöchl, P. E. Projector augmented-wave method. Phys Rev B 1994, 50, 17953.

(43) Kresse, G.; Joubert, D. From ultrasoft pseudopotentials to the projector augmented-wave method. Phys Rev B 1999, 59, 1758.

(44) Liechtenstein, A. I.; Anisimov, V. I.; Zaanen, J. Density-functional theory and strong interactions: Orbital ordering in Mott-Hubbard insulators. Phys Rev B 1995, 52, R5467.

(45) Madsen, G. K. H.; Singh, D. J. BoltzTraP. A code for calculating bandstructure dependent quantities. Computer Physics Communications 2006, 175, 67. (46) Parlinski, K.; Li, Z. Q.; Kawazoe, Y. First-principles determination of the soft mode in cubic ZrO2. Phys Rev Lett 1997, 78, 4063.

(47) Togo, A.; Tanaka, I. First principles phonon calculations in materials science. Scripta Mater 2015, 108, 1.

(48) Zhang, H. J.; Lee, G.; Cho, K. Thermal transport in graphene and effects of vacancy defects. Phys Rev B 2011, 84, 115460.

(49) Yildirim, T.; Gulseren, O.; Lynn, J. W.; Brown, C. M.; Udovic, T. J.; Huang, Q.; Rogado, N.; Regan, K. A.; Hayward, M. A.; Slusky, J. S.; He, T.; Haas, M. K.; Khalifah, P.; Inumaru, K.; Cava, R. J. Giant anharmonicity and nonlinear electron-phonon coupling in $\mathrm{MgB} 2$ : A combined first-principles calculation and neutron scattering study. Phys Rev Lett 2001, 87, 037001. 


\section{CHAPTER SEVEN: HETEROSTRUCTURE EFFECTS ON STRUCTURAL, ELECTRICAL, AND PHONON PROPERTIES OF TWO-DIMENSIONAL TRANSITION METAL DICHALCOGENIDES}

The formation of a lateral heterostructure between 2D TMDs has promising optical and electronic applications and remains a relatively unexplored area for materialsby-design. ${ }^{1}$ First-principles calculations ${ }^{2}$ and experimental results ${ }^{3,4}$ show a type II (staggered) band alignment between $2 \mathrm{D} \mathrm{MoS}_{2}$ and $\mathrm{WS}_{2}$ which has potential for optoelectronic applications. In this chapter, the heterostructure interface between 2D $\mathrm{MoS}_{2}$ and $2 \mathrm{D} \mathrm{MS} 2(\mathrm{M}=\mathrm{Cr}, \mathrm{Sc}, \mathrm{Ti}$, and $\mathrm{W})$ are investigated for structural, energetic, electronic, and phonon effects. The physical features of the transition metals along with the geometric and electronic structures of the pure constituent 2D TMDs play large roles in the stability of the heterostructure and the interfacial properties.

\subsection{Computational Methods}

Structural and energetic calculations were performed using VASP within the framework of DFT. ${ }^{5}$ PAW pseudopotentials ${ }^{6,7}$ were used along with spin-dependent GGA exchange correlation functionals defined by the PBE formalism. ${ }^{8}$ The plane-wave basis sets were expanded to a cutoff of $400 \mathrm{eV}$. Fermi surface broadening was handled with a Gaussian smearing of $0.05 \mathrm{eV}$ and structures were relaxed until residual forces were reduced to $0.01 \mathrm{eV} / \AA$. To replicate the ideal $2 \mathrm{D}$ structure and avoid spurious image interaction, a vacuum space $>16 \AA$ was inserted between periodic sheets. $\mathrm{DFT}+\mathrm{U}^{9}$ was 
employed to handle strong correlation effects in the electronic structure calculations. Onsite Coulomb potentials of $\mathrm{U}=5.0,4.38,3.0,8.0$, and $8.0 \mathrm{eV}$ were used for $\mathrm{Cr}$, Mo, Sc, $\mathrm{Ti}$, and $\mathrm{W}$, respectively, while $1.0 \mathrm{eV}$ was used for all $\mathrm{J}$ values. Brillouin zone integration was handled using a $\Gamma$-centered $3 \times 9 \times 1 k$-point mesh for electronic structure calculations.

To calculate the phonon properties of a material, second-order force constants must first be generated. This was handled using DFPT within the VASP code.$^{10}$ These force constants were then used to generate phonon DoS plots ${ }^{11}$ and used as inputs into the Atomistic Green's Function (AGF) code. The AGF code calculates the phonon transmission function $\Xi(\omega)$ for the interface of two materials and the transmission coefficient for individual phonon modes. To calculate the phonon transmission function, the system is partitioned into three components: the semi-infinite left lead, the interface region and the semi-infinite right lead. The AGF describes phonon propagation across the interface region and is calculated from the force constant matrices associated with the aforementioned components. The transmission function $\Theta(\omega)$, which corresponds to the total phonon transmission across the interface region, is computed from the AGF and depends on the phonon dispersion of the leads and the atomistic structure of the interface. Given $\Theta(\omega)$, the thermal boundary conductance (TBC) can be computed using the following expression ${ }^{12}$

$$
G(T)=\frac{1}{2 \pi} \int_{0}^{\infty} d \omega \hbar \omega \frac{d N(\omega, T)}{d T} \Theta(\omega)
$$

where $N$ and $T$ are the Bose-Einstein function and the temperature, respectively. In addition, recent extensions to the AGF method by Ong and Zhang ${ }^{12}$ have also enabled the efficient computation of transmission coefficients for individual phonon modes. These extensions allow for a more detailed and deeper analysis of the distribution of transmitted 
phonon modes and its connection to the phonon dispersion of the constituent materials on both sides of the interface.

To minimize error in this chapter, DFT input parameters were optimized through convergence tests similar to that of Sections 4.2, 5.2, and 6.2. The only literature values available for result validation came from pure $\mathrm{MS}_{2}$ band alignments ${ }^{13,14}$ and $\mathrm{MoS}_{2}-\mathrm{WS}_{2}$ heterostructure bandgap type. ${ }^{15}$

\subsection{Results}

To broaden the understanding of 2D TMD heterostructures and the factors that contribute to their structural stability, electronic properties, and phonon properties, each result in this work was compared to the physical features of the transition metals. Similar to our previous work, ${ }^{16}$ we focused on the most common oxidation state, atomic masses ${ }^{17}$ and atomic radii ${ }^{18}$ of the individual transition metals. By comparing these physical features between Mo and M, and analyzing their effects on structure, electronic, and phonon properties, we identified key parameters that govern 2D TMD lateral heterostructures.

\section{$\underline{\text { Structural Features }}$}

DFT-based calculations were conducted to determine the structural effects of forming a heterostructure between $2 \mathrm{D} \mathrm{MoS} 2$ and $2 \mathrm{D} \mathrm{MS} 2(\mathrm{M}=\mathrm{Cr}, \mathrm{Sc}, \mathrm{Ti}$, and $\mathrm{W})$, see Figure 7.1Figure 7.. Table 7.1 lists the structural mismatch values, $\Delta a$ and $\Delta d_{S-s}$, between adjacent structures. Each of these values were derived from the pure 2D TMD lattice constant, $a$, and S-S separation distance, $d_{S-S}$ (refer to Figure 6.2), according to the following equations.

$$
\Delta a=\left|\frac{a_{M o S 2}-a_{M S 2}}{a_{M o S 2}}\right| * 100 \%
$$




$$
\Delta d_{S-S}=\left|\frac{d_{S-S}^{M o S}-d_{S-S}^{M S 2}}{d_{S-S}^{M o S 2}}\right| * 100 \%
$$

The W system has the lowest $\Delta a$ and $\Delta d_{S-S}$ values meaning its interface will have the least amount of strain compared to the other transition metals in this work. The $\mathrm{Cr}$ and $\mathrm{Ti}$ systems each had similar $\Delta a$ values and the $\mathrm{Cr}$ had lower $\Delta d_{S-S}(6.09 \%$ compared to $8.74 \%$ ) so the $\mathrm{MoS}_{2}-\mathrm{CrS}_{2}$ interface has slightly less strain than that of the $\mathrm{MoS}_{2}-\mathrm{TiS}_{2}$.

The pure $\mathrm{ScS}_{2}$ system has a large difference in lattice constant and $\mathrm{S}-\mathrm{S}$ distance compared to $\mathrm{MoS}_{2}$ and likewise has the largest mismatch values. Sc has the lowest atomic mass and largest atomic radius of all transition metals investigated. However, compared to the features of Mo, $\mathrm{W}$ has a larger mass difference and $\mathrm{Cr}$ has a larger radius difference than Sc suggesting that the size and mass of the metal are not the critical features for minimizing mismatch. The Sc does have the largest difference in oxidation state $(+3$ compared to Mo's +6) which is likely a dominant factor for finding a stable heterostructure.

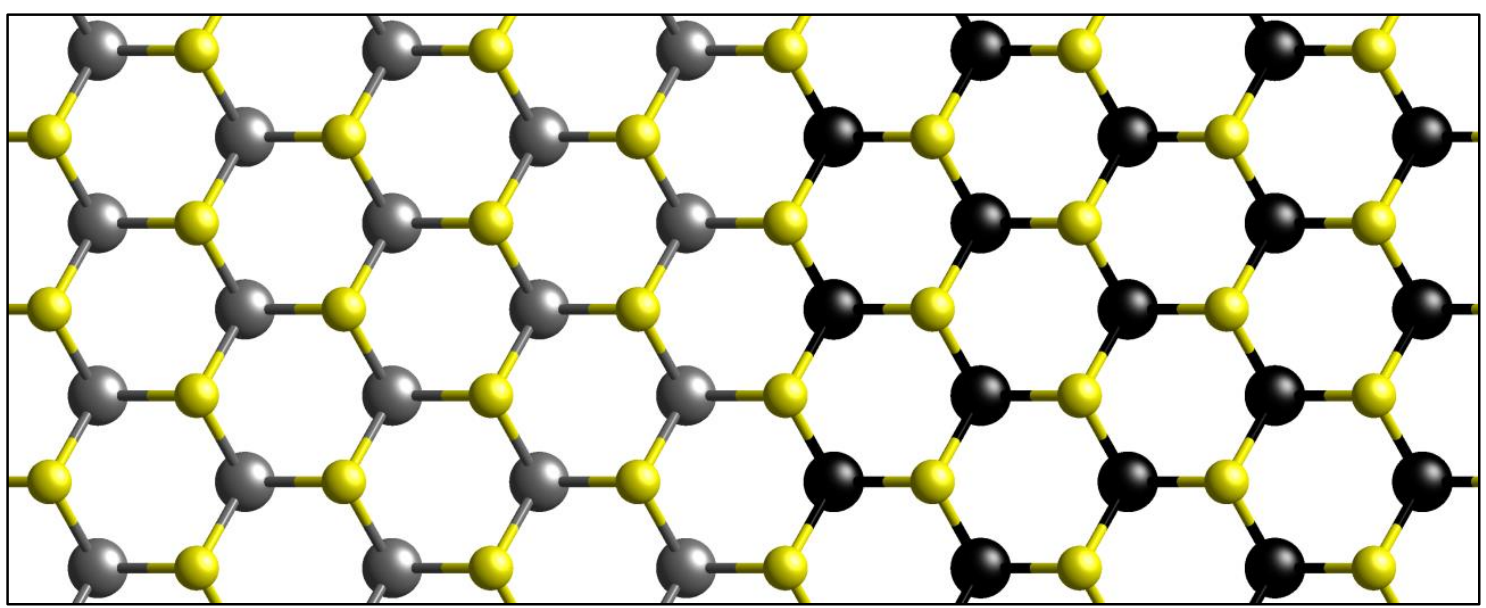

Figure 7.1. Schematic of the $\mathrm{MoS}_{2}-\mathrm{MS}_{2}(\mathrm{M}=\mathrm{Cr}, \mathrm{Sc}, \mathrm{Ti}$, or W) heterostructure where the Mo, S, and $M$ atoms are depicted using gray, yellow, and black spheres, respectively 
Table 7.1. Physical features of the transition metals, $\mathrm{M}$, in $2 \mathrm{D} \mathrm{MoS}_{2}-\mathrm{MS}_{2}$ lateral heterostructures. The lattice, $\Delta a$, and S-S distance, $\Delta d_{S-S}$, mismatches are based off of the pure 2D MoS 2 and $\mathrm{MS}_{2}$ structures. The formation energy and electronic bandgap of the heterostructure is given as $E_{f}$ and $E_{g}$, respectively.

\begin{tabular}{|c|c|c|c|c|c|c|c|}
\hline \multicolumn{4}{|c|}{$\mathbf{M}$} & \multicolumn{2}{|c|}{ Mismatch } & \multirow[b]{2}{*}{$E_{f}(\mathrm{eV})$} & \multirow[b]{2}{*}{$E_{g}(\mathrm{eV})$} \\
\hline symbol & $\begin{array}{c}\text { common } \\
\text { oxidation } \\
\text { state }\end{array}$ & $\begin{array}{c}\text { atomic } \\
\operatorname{mass}^{\mathrm{a}}(\mathrm{u})\end{array}$ & $\begin{array}{c}\text { atomic } \\
\text { radius }^{\mathrm{b}}(\AA)\end{array}$ & $\Delta a$ & $\Delta d_{S-S}$ & & \\
\hline $\mathrm{Cr}$ & +6 & 51.996 & 0.69 & $4.44 \%$ & $6.09 \%$ & -0.02 & 0.97 \\
\hline $\mathrm{Sc}$ & +3 & 44.956 & $0.885^{\mathrm{c}}$ & $18.73 \%$ & $13.42 \%$ & 4.29 & -- \\
\hline $\mathrm{Ti}$ & +4 & 47.88 & 0.745 & $4.80 \%$ & $8.74 \%$ & 1.54 & -- \\
\hline W & +6 & 183.85 & 0.80 & $0.03 \%$ & $0.38 \%$ & -0.32 & 1.59 \\
\hline Mo & +6 & 95.94 & 0.79 & & & & \\
\hline
\end{tabular}

${ }^{\mathrm{a}}$ Reference [14]

${ }^{\mathrm{b}}$ Reference [15]. Values taken from crystal radii with +4 charge and 6-fold coordination. ${ }^{c}$ Value for +3 charge

To analyze the thermodynamic stability of these heterostructures, the formation energy, $E_{f}$, was calculated for each system. This was done according to the following equation:

$$
E_{f}=E_{t o t}^{h e t}-n_{u c}^{M o S 2} E_{u c}^{M o S 2}-n_{u c}^{M S 2} E_{u c}^{M S 2}
$$

where $E_{\text {tot }}^{\text {het. }}$ is the total energy of the $\mathrm{MoS}_{2}-\mathrm{MS}_{2}$ heterostructure, $E_{u c}^{M o S 2}$ and $E_{u c}^{M S 2}$ are the energies of a single unit cell of pure $2 \mathrm{D} \mathrm{MoS} 2$ and $\mathrm{MS}_{2}$, respectively, and $n_{u c}^{M o S 2}$ and $n_{u c}^{M S 2}$ are the number of unit cells of each material present in the heterostructure. The system with the largest $E_{f}$ value is the Sc with $4.29 \mathrm{eV}$. This indicates that the individual pure substances are more stable than the heterostructure, which requires energy in order to form. This is expected given the large difference in oxidation state between $\mathrm{Mo}$ and $\mathrm{Sc}$ as well as large mismatch in both lattice constant and S-S distance. With a negligible $E_{f}$ value, the $\mathrm{MoS}_{2}-\mathrm{CrS}_{2}$ heterostructure is predicted to be stable whereas $\mathrm{MoS}_{2}-\mathrm{TiS}_{2}$, which 
has an $E_{f}$ of $1.54 \mathrm{eV}$, is not. With relatively similar mismatch values and atomic mass and weight, the improved stability of the Cr system is likely due to its oxidation state. The only heterostructures that do not yield positive $E_{f}$ values are $\mathrm{Cr}$ and $\mathrm{W}$ which are also those that have the same oxidation state as Mo (+6). These results indicate that the formation of stable $2 \mathrm{D} \mathrm{MS}$ lateral heterostructures is strongly dependent on the oxidation states between transition metals in two compositions.

\section{Electronic Properties}

Electronic DoS calculations were performed for each $\mathrm{MoS}_{2}-\mathrm{MS}_{2}(\mathrm{M}=\mathrm{Cr}, \mathrm{Sc}, \mathrm{Ti}$, and $\mathrm{W}$ ) heterostructure and their bandgaps are listed in Table 7.1. The two most stable structures, the systems having $\mathrm{Cr}$ and $\mathrm{W}$, are also the only heterostructures that have a bandgap. A more detailed view is provided in Figure 7.2 which depicts the electronic band structure diagrams for the $\mathrm{Cr}$ and $\mathrm{W}$ systems. The VBM for both structures is similar while the CBM for the $\mathrm{W}$ system is about $0.6 \mathrm{eV}$ higher. The $\mathrm{MoS}_{2}-\mathrm{WS}_{2}$ heterostructure is also a direct bandgap semiconductor with VBM and CBM both occurring at the $\Gamma$ point. Alternatively, the $\mathrm{CBM}$ in the $\mathrm{MoS}_{2}-\mathrm{CrS}_{2}$ system shifts away from the $\Gamma$ point (as indicated by the blue arrow in Figure 7.2) towards the K point. This transition from direct to indirect semiconducting behavior must originate from the interface since the pure $2 \mathrm{D} \mathrm{MoS}, \mathrm{WS}_{2}$, and $\mathrm{CrS}_{2}$ are all direct bandgap semiconductors. ${ }^{14,16}$ 

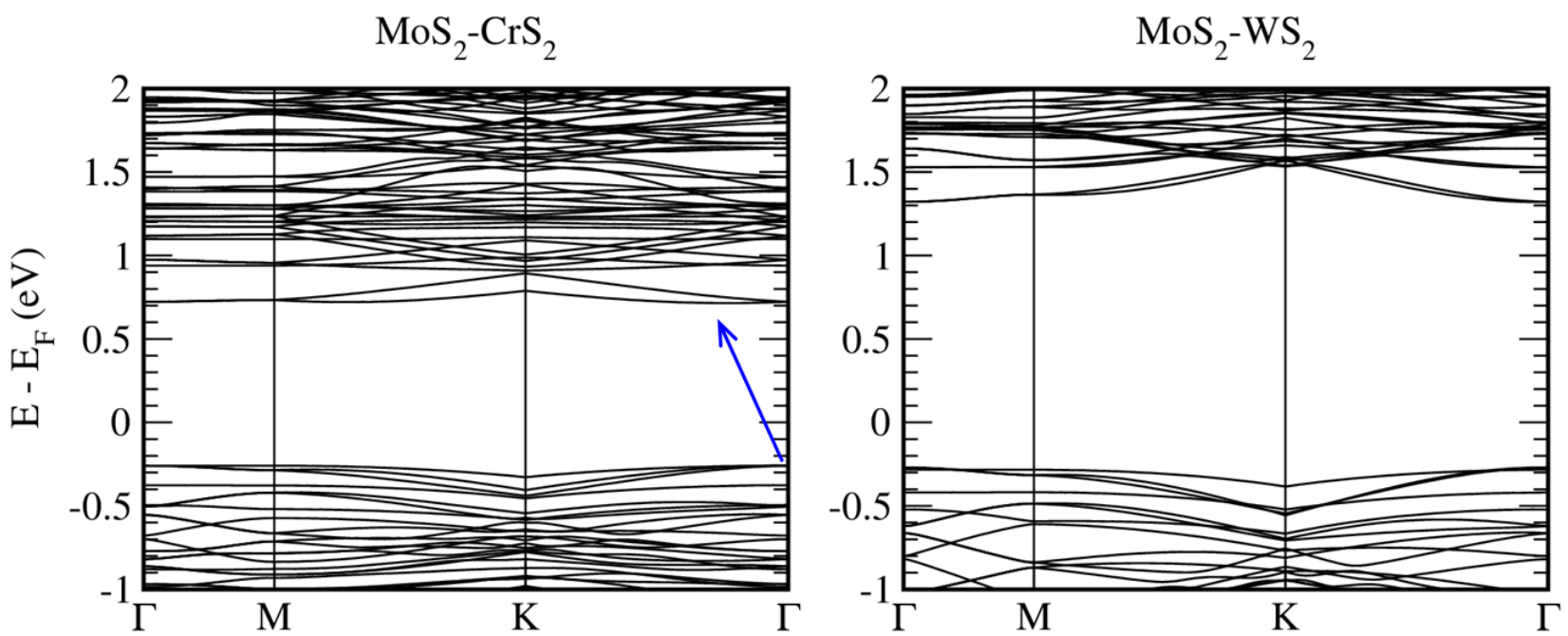

Figure 7.2. Electronic band structure diagrams for $\mathrm{MoS}_{2}-\mathrm{CrS}_{2}$ and $\mathrm{MoS}_{2}-\mathrm{WS}_{2}$ lateral heterostructures. The $y$-axis for both plots has been shifted to $E_{F}=0 \mathrm{eV}$. The $\mathrm{MoS}_{2}-W_{2}$ structure is determined to be a direct semiconductor at the $\Gamma$ point whereas the lowest conduction band in the $\mathrm{MoS}_{2}-\mathrm{CrS}_{2}$ structure has shifted slightly towards the $K$ point exhibiting indirect semiconducting behavior (as indicated by the blue arrow).

The bandgap for the $\mathrm{MoS}_{2}-\mathrm{CrS}_{2}$ system $(0.97 \mathrm{eV})$ is similar to that of the pure 2D $\mathrm{CrS}_{2}$ compound $(0.95 \mathrm{eV})$ while the $\mathrm{MoS}_{2}-\mathrm{WS}_{2}$ system $(1.59 \mathrm{eV})$ is smaller than either the pure $2 \mathrm{D} \mathrm{MoS}_{2}(1.69 \mathrm{eV})$ or pure $2 \mathrm{D} \mathrm{WS} 2(1.85 \mathrm{eV})$. To clarify this, the band alignments for each of the pure $2 \mathrm{D} \mathrm{MS}$ 2 systems were plotted in Figure 7.3. The VBM for both $\mathrm{CrS}_{2}$ and $\mathrm{MoS}_{2}$ are about $-5.80 \mathrm{eV}$ while the $\mathrm{CBM}$ of the $\mathrm{CrS}_{2}$ is significantly lower than that of the $\mathrm{MoS}_{2}(-4.84 \mathrm{eV}$ compared to $-4.13 \mathrm{eV})$. This suggests that the $\mathrm{CrS}_{2}$ layer in the heterostructure contributes more to the CBM than the $\mathrm{MoS}_{2}$ layer. It also explains how the bandgap for $\mathrm{MoS}_{2}-\mathrm{CrS}_{2}$ heterostructure is limited by the bandgap of the pure $\mathrm{CrS}_{2}$. The $\mathrm{MoS}_{2}-\mathrm{WS}_{2}$ heterostructure exhibits type II (staggered) band alignment with a bandgap of $1.58 \mathrm{eV} .^{2,15}$ This means that the CBM and VBM of one system are each higher than that of the adjacent system. In this case, the CBM/VBM levels for $\mathrm{WS}_{2}(-$ 3.50/-5.35 eV) are each higher than that of $\mathrm{MoS}_{2}(-4.13 /-5.82 \mathrm{eV})$. The VBM of the resulting heterostructure is contributed mostly from the $\mathrm{WS}_{2}$ layer while the CBM is 
contributed from the $\mathrm{MoS}_{2}$ layer. This results in a reduced bandgap for the heterostructure compared to the individual layers. The difference between the pure $\mathrm{WS}_{2}$ $\operatorname{VBM}(-5.35 \mathrm{eV})$ and the pure $\mathrm{MoS}_{2} \mathrm{CBM}(-4.13 \mathrm{eV})$ is only $1.22 \mathrm{eV}$, so it was expected that the calculated bandgap of the heterostructure would be around $1.22 \mathrm{eV}$. However, we calculated a bandgap for the $\mathrm{MoS}_{2}-\mathrm{WS}_{2}$ heterostructure of $1.59 \mathrm{eV}$. The orbital overlap for the interface is likely different from that of the pure substances - which have a CBM and VBM originating from the repulsion between anion $p$ and cation $d$ orbitals. ${ }^{15}$ The bonding character at the interface plays a crucial role in the electrical properties of the heterostructure. Understanding the correlation between physical features of constituent atoms and the resulting 2D TMD band alignments can guide materials design. 


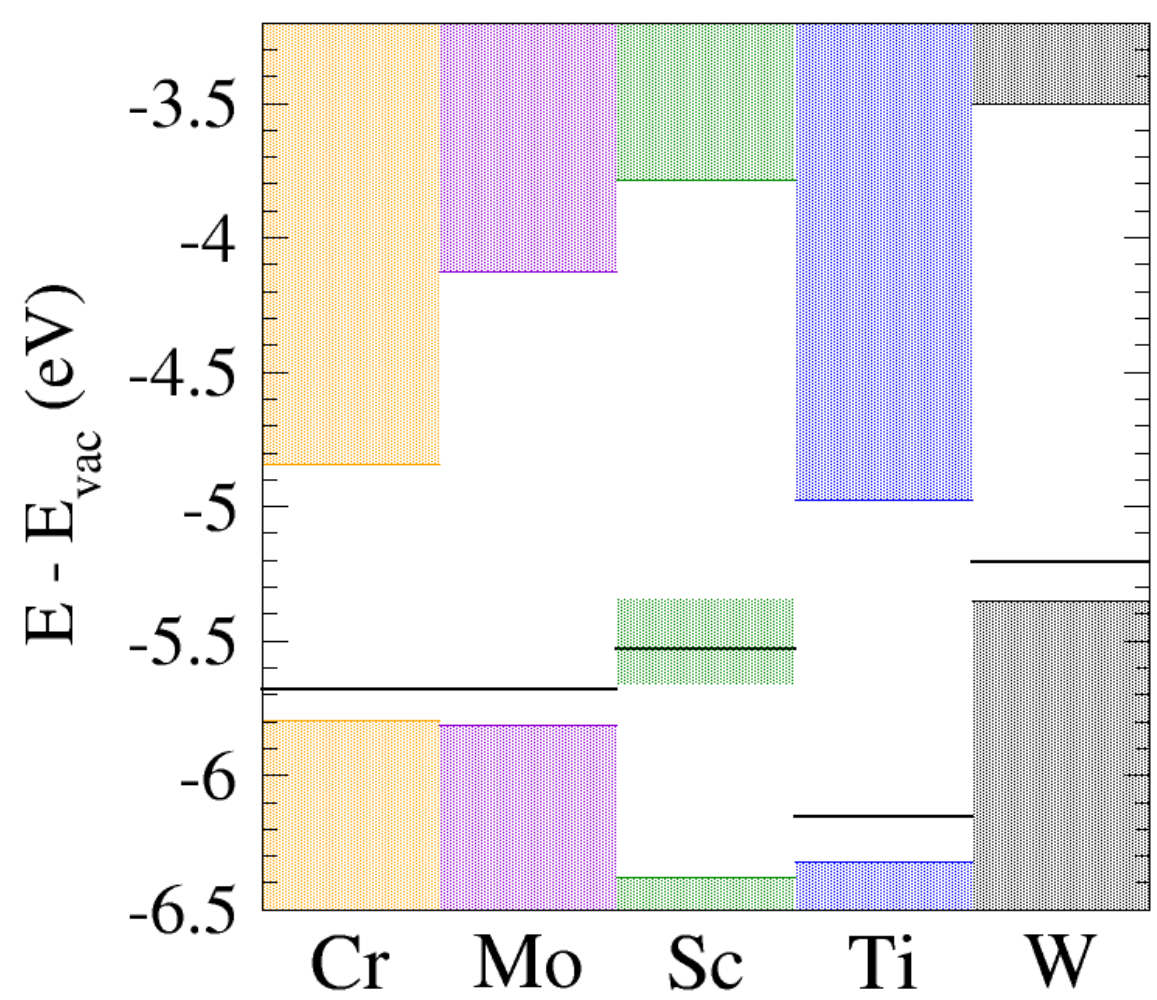

Figure 7.3. Band alignments for each of the pure 2D $\mathrm{MS}_{2}(\mathrm{M}=\mathrm{Cr}, \mathrm{Mo}, \mathrm{Sc}, \mathrm{Ti}$, and W) structures shifted to the vacuum level. Horizontal black lines represent the Fermi level $\left(E_{F}\right)$ for each structure.

\section{$\underline{\text { Phonon Properties }}$}

Phonon DoS spectra, plotted in Figure 7.4, reveals that the Sc and Ti systems have significant negative (imaginary) phonon frequencies suggesting instability in the structures. These findings are consistent with our $E_{f}$ results in Table 7.1. Alternatively, the $\mathrm{Cr}$ and $\mathrm{W}$ heterostructures have negligible negative frequencies and are stable. The $\mathrm{Cr}$ has the highest-frequency optical bands among the heterostructures investigated in this work. The $\mathrm{W}$ has sharper features and is the only system that has a significant gap between acoustic and optical phonon bands. Previous first-principles calculations have shown a correlation between phonon frequency gaps and high thermal conductivity. ${ }^{19}$ This is attributed to fewer and weaker scattering events between acoustic and optical phonon modes that have a large separation in frequency. By this reasoning, the Cr system 
which has no frequency gap would be expected to have greater acoustic-optical phonon scattering than $\mathrm{W}$. The calculation of third-order force constants would be required to estimate lattice thermal conductivity and confirm this observation.

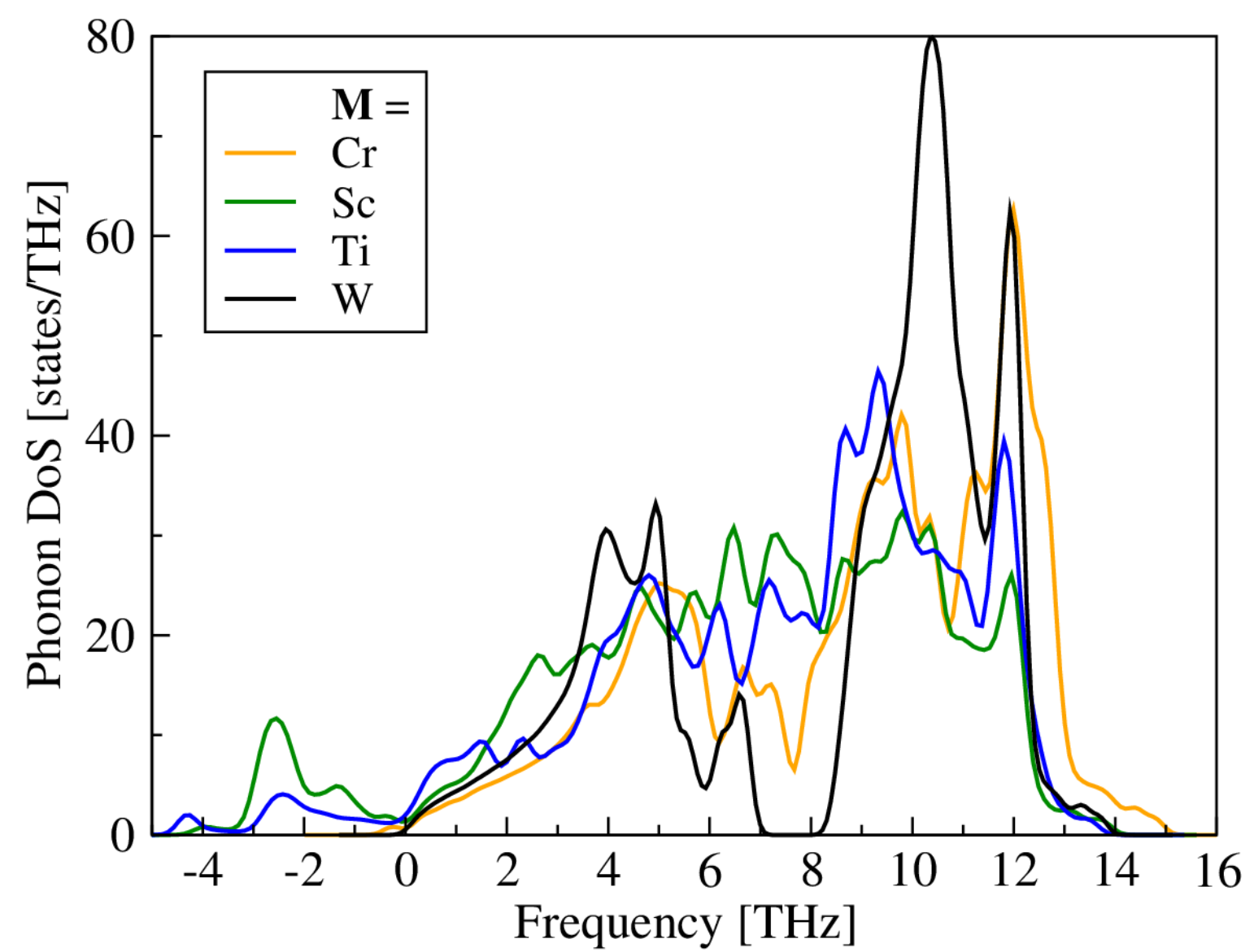

Figure 7.4. Phonon density of states calculated for each 2D $\mathrm{MoS}_{2}-\mathrm{MS}_{2}$ heterostructure where the yellow $(\mathrm{Cr})$, green $(\mathrm{Sc})$, blue (Ti), and black $(\mathrm{W})$ plots represent each respective transition metal, $M$. Negative (imaginary) phonon frequencies are found for the two least stable (lowest formation energy) heterostructures: $\mathrm{M}=\mathrm{Sc}$ and $\mathrm{Ti}$.

\section{Atomistic Green's Function (AGF) Results}

Another approach for determining the thermal effects of a heterostructure is through the investigation of the interfacial thermal resistance. This is dependent on the transmission of phonons across the heterojunction. We use the extended AGF to investigate interfacial thermal transport at the $\mathrm{MoS}_{2}-\mathrm{WS}_{2}$ interface and the underlying 
phonon mechanisms. Using second-order force constants as input into the AGF code, the phonon transmission coefficients can be calculated for each phonon mode in the first Brillouin zone. Each data point in Figure 7.5, which shows the transmission coefficient spectra for the acoustic phonons in $\mathrm{MoS}_{2}$ and $\mathrm{WS}_{2}$, represents a phonon mode while the color indicates the transmission coefficient or fraction of energy transmitted across the interface. The gray-shaded points correspond to phonon modes that do not contribute to the interfacial head flux because their group velocities are in the direction away from the interface. Most of the transmission in this heterostructure is contributed by the acoustic phonon modes so, for the sake of brevity, only the transmissions from the three acoustic modes are provided. The left column in Figure 7.5 represents the rightward interfacial heat flux contribution from $\mathrm{MoS}_{2}$ phonons while the right column represents the leftward interfacial heat flux contribution from $\mathrm{WS}_{2}$ phonons. 

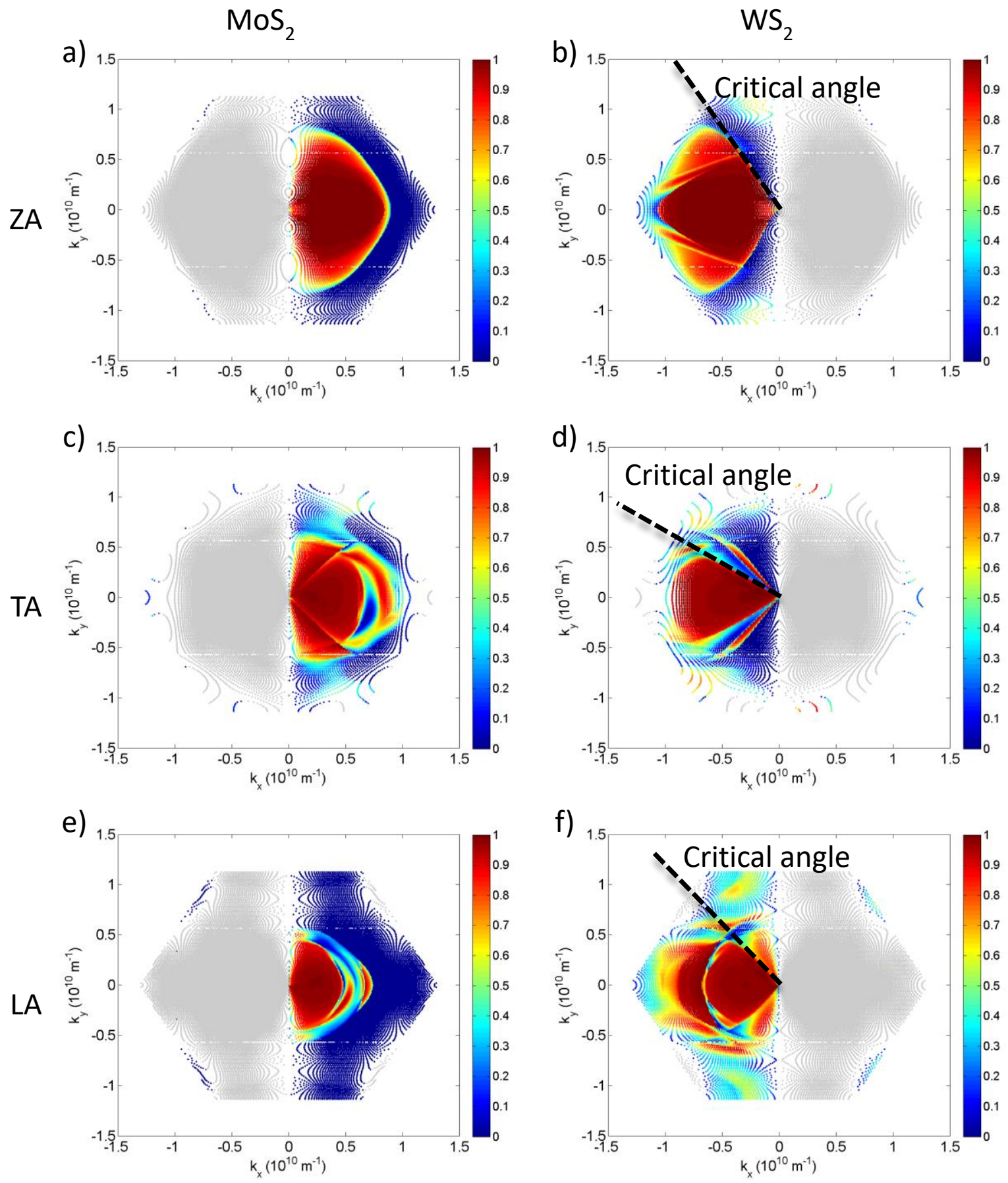

Figure 7.5. Phonon transmission coefficients across the $\mathrm{MoS}_{2}-\mathrm{WS}_{2}$ interface for the ZA (a and b), TA (c and d), and LA (e and f) acoustic phonon modes. The left column (a, c, and e) represents $\mathrm{MoS}_{2}$ phonons contributing to leftward interfacial heat flux whereas the right column (b, $d$, and f) represents $W_{2}$ phonons contributing to rightward interfacial heat flux. 
In general, the transmission coefficients converge to unity when the wave vectors for the phonons are small, corresponding to long wavelengths and low frequencies. This implies that the interface is highly permeable to the low-frequency, long-wavelength acoustic phonons. However, as we move radially away from the center of the Brillouin zone towards the zone boundaries, the phonon frequencies increase and the associated phonon transmission coefficients become smaller since higher-frequency phonons are strongly scattered by the interface. In addition, a 'critical angle' is observed for phonon transmission from the $\mathrm{WS}_{2}$ phonons while no such phenomenon is observed for $\mathrm{MoS}_{2}$ phonons. Beyond this angle, there is no transmission on the $\mathrm{WS}_{2}$ side. This manifestation of the critical angle is analogous to the critical angle in the refraction of light between two media of different refractive indices and is due to the lower acoustic phonon group velocity in $\mathrm{WS}_{2}$ which is denser because of the heavier $\mathrm{W}$ atoms. The transmission coefficient also depends on the frequency alignment of phonons with similar polarizations in $\mathrm{MoS}_{2}$ and $\mathrm{WS}_{2}$. In $\mathrm{MoS}_{2}$, because its phonon velocities are generally higher, a wider angular distribution of phonon modes contribute to the heat flux while in $\mathrm{WS}_{2}$, a lower angular distribution of phonon modes are involved in interfacial thermal transport because of the critical angle. Although, a larger portion of the phonon modes with the arc defined by that critical angle are transmitted across the interface.

The TBC, $G$, relates the interfacial heat flux, $Q$, to the temperature drop across a boundary, $\Delta \mathrm{T}$, according to the following equation:

$$
Q=G \Delta T
$$

For large values of $G$, the temperature drop across the boundary is small. In Figure 7.6, the total $\mathrm{TBC}$ is plotted as a function of temperature up to $500 \mathrm{~K}$. We note that the TBC 
increases as a function of temperature because more phonon modes become populated and contribute to interfacial thermal transport at higher temperatures. One advantage of our use of the extended AGF method is that it also allows us to distinguish and quantify the contribution of the phonons by their band indices. In $\mathrm{MoS}_{2}$ and $\mathrm{WS}_{2}$, there are 3 atoms in the primitive unit cell and thus a total of $3 \times 3=9$ phonon bands $(3$ acoustic and 6 optical) since each band corresponds to an atomic degree of freedom. Acoustic phonons are expected to contribute more disproportionately to interfacial thermal transport because they have much higher group velocities and are more easily transmitted across the interface. To verify this, we computed the TBC from only the acoustic phonons and from the acoustic and optical phonons (see Figure 7.6). The contribution from the acoustic phonon modes dominates the TBC for all temperatures. At room temperature $(300 \mathrm{~K})$, the contribution from the optical phonons, which accounts for the difference between the two curves in Figure 7.6, only comprises about $16 \%$ of the TBC, confirming the commonly held assumption that interfacial heat flux is primarily mediated by acoustic phonons. ${ }^{20}$ Nonetheless, the optical phonon contribution is not insignificant and can be a source of discrepancy if not included in the computation of the TBC, especially at high temperatures. We also note that the TBC for the $\mathrm{MoS}_{2}-\mathrm{WS}_{2}$ interface is much lower than that of the graphene/ $h$-BN interface. ${ }^{12,21}$ At room temperature, the $\mathrm{MoS}_{2}-\mathrm{WS}_{2}$ TBC is $\sim 351 \mathrm{MWK}^{-1} \mathrm{~m}^{-2}$ while the graphene/ $h$-BN TBC is $2.5 \mathrm{GWK}^{-1} \mathrm{~m}^{-2}$, almost an order of magnitude larger. This is due to the lower acoustic phonon velocities in $\mathrm{MoS}_{2}$ and $\mathrm{WS}_{2}$, which limit their maximum interfacial heat flux, compared to graphene and $h$-BN.

The use of this extended AGF method allows for a thorough analysis of the heat flux across a heterojunction and can further the understanding of transport properties 
within these nanostructures. The expansion of this technique towards other 2D TMD heterostructures will be crucial to develop these materials for energy applications.

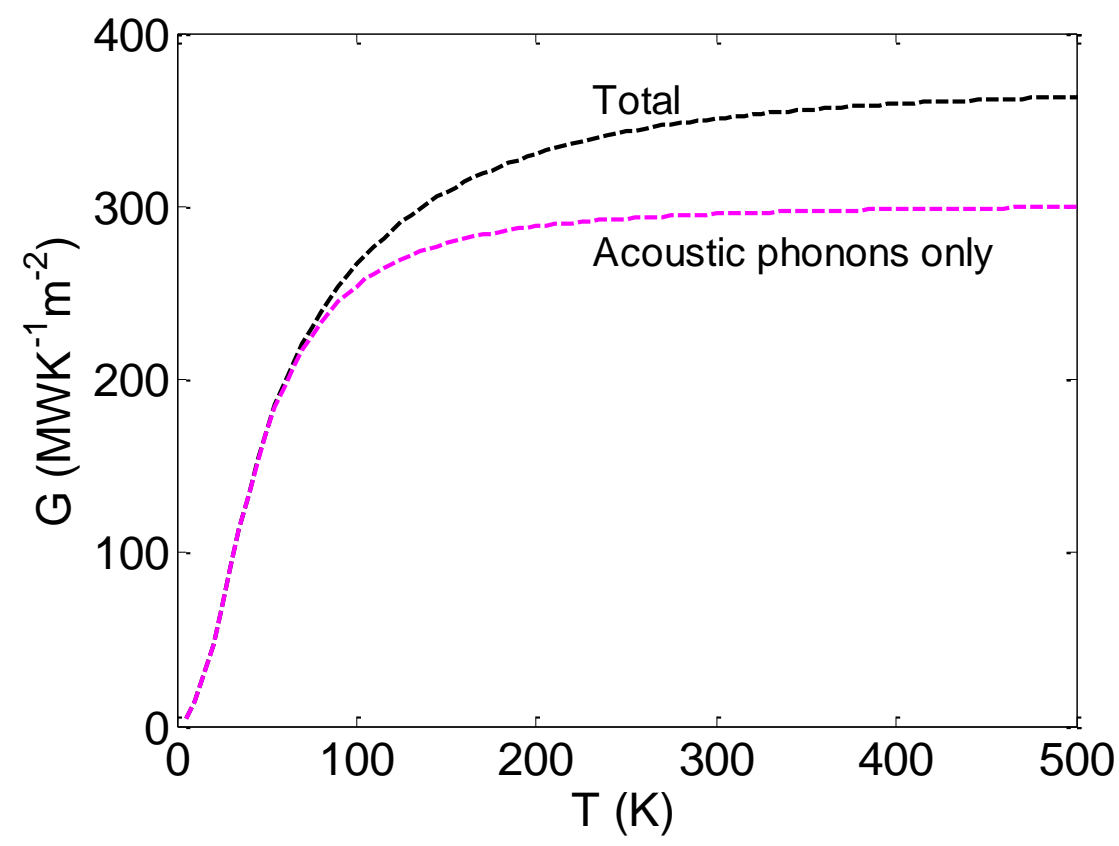

Figure 7.6. Thermal boundary conductance (TBC) for $\mathrm{MoS}_{2}-\mathrm{WS}_{2}$ lateral interface. The dotted black plot represents the total TBC for the system and the dotted pink plot represents the TBC contribution from the acoustic phonon modes.

\subsection{Conclusion}

DFT calculations were performed to analyze the structural, energetic, electronic, and phonon effects of a lateral heterostructure formation between $2 \mathrm{D} \mathrm{MoS}$ and 2D $\mathrm{MS}_{2}$ $(\mathrm{M}=\mathrm{Cr}, \mathrm{Sc}, \mathrm{Ti}$, or $\mathrm{W})$. The choice of transition metals having similar oxidation states had a much greater effect on the stability of the heterostructure than the atomic mass, atomic radius, or mismatch between the TMDs $\left(\Delta a\right.$ or $\left.\Delta d_{s-S}\right)$. The $\mathrm{MoS}_{2}-\mathrm{ScS}_{2}$ and $\mathrm{MoS}_{2-}$ $\mathrm{TiS}_{2}$ heterostructures were determined to be the least stable systems due to their positive formation energies $\left(E_{f}\right)$ and negative phonon frequencies. The $E_{f}$ values for both the $\mathrm{MoS}_{2}-\mathrm{CrS}_{2}$ and $\mathrm{MoS}_{2}-\mathrm{WS}_{2}$ heterostructures are low and neither exhibit significant negative phonon frequencies, suggesting that both heterostructures are stable. The $\mathrm{Cr}$ and 
W systems are also the only systems to exhibit an electronic bandgap. The heterostructure bandgaps are determined by the VBM and CBM values from the constituent pure TMDs. The VBMs for both pure $\mathrm{CrS}_{2}$ and $\mathrm{MoS}_{2}$ are aligned but the $\mathrm{CrS}_{2} \mathrm{CBM}$ is lower than the $\mathrm{MoS}_{2}$, so the resulting heterostructure has a bandgap that matches that of the pure $\mathrm{CrS}_{2}$. However, the $\mathrm{MoS}_{2}-\mathrm{WS}_{2}$ forms a type II (staggered) alignment so the resulting heterostructure bandgap is smaller than that of either TMD. The $\mathrm{MoS}_{2}-\mathrm{WS}_{2}$ phonon DoS was found to be the only system with a frequency gap between acoustic and optical bands, suggesting that it experiences the least amount of acoustic-optical phonon scattering among these heterostructures.

Our simulation of the thermal boundary conductance at the $\mathrm{MoS}_{2}-\mathrm{WS}_{2}$ interface shows that the $\mathrm{TBC}$ is dominated by the acoustic phonons and primarily limited by the differences in acoustic phonon velocities between $\mathrm{MoS}_{2}$ and $\mathrm{WS}_{2}$. Our results also indicate that the optical phonons have a small but significant contribution to the TBC especially at high temperatures. Compared to the graphene/ $h-\mathrm{BN}$ interface, our calculated TBC for the $\mathrm{MoS}_{2}-\mathrm{WS}_{2}$ system is almost an order of magnitude smaller. This is due to the lower acoustic phonon velocities in $\mathrm{MoS}_{2}$ and $\mathrm{WS}_{2}$. This may potentially be a source of impediment to efficient heat dissipation in nanoelectronic devices with such interfaces.

\section{Acknowledgements}

The authors would like to thank Jason Cook, Tyler Bevan, and Cormac Garvey for technical support. High performance computing facilities were provided by Boise State University's R1 computing cluster and the Idaho National Laboratory's high performance computing (HPC) center. 


\section{References}

(1) Wang, H. T.; Yuan, H. T.; Hong, S. S.; Li, Y. B.; Cui, Y. Physical and chemical tuning of two-dimensional transition metal dichalcogenides. Chem Soc Rev 2015, 44, 2664.

(2) Wang, Q. W.; Wu, P.; Cao, G. Y.; Huang, M. First-principles study of the structural and electronic properties of MoS2-WS2 and MoS2-MoTe2 monolayer heterostructures. J Phys D Appl Phys 2013, 46, 505308.

(3) Huang, C. M.; Wu, S. F.; Sanchez, A. M.; Peters, J. J. P.; Beanland, R.; Ross, J. S.; Rivera, P.; Yao, W.; Cobden, D. H.; Xu, X. D. Lateral heterojunctions within monolayer MoSe2-WSe2 semiconductors. Nature Materials 2014, 13, 1096.

(4) Chen, K.; Wan, X.; Wen, J. X.; Xie, W. G.; Kang, Z. W.; Zeng, X. L.; Chen, H. J.; Xu, J. B. Electronic Properties of MoS2-WS2 Heterostructures Synthesized with Two-Step Lateral Epitaxial Strategy. Acs Nano 2015, 9, 9868.

(5) Kresse, G.; Furthmuller, J. Efficient iterative schemes for ab initio totalenergy calculations using a plane-wave basis set. Phys Rev B 1996, 54, 11169.

(6) Blöchl, P. E. Projector augmented-wave method. Phys Rev B 1994, 50, 17953.

(7) Kresse, G.; Joubert, D. From ultrasoft pseudopotentials to the projector augmented-wave method. Phys Rev B 1999, 59, 1758.

(8) Perdew, J. P.; Burke, K.; Ernzerhof, M. Generalized gradient approximation made simple. Phys Rev Lett 1996, 77, 3865.

(9) Liechtenstein, A. I.; Anisimov, V. I.; Zaanen, J. Density-functional theory and strong interactions: Orbital ordering in Mott-Hubbard insulators. Phys Rev B 1995, 52, R5467.

(10) Parlinski, K.; Li, Z. Q.; Kawazoe, Y. First-principles determination of the soft mode in cubic ZrO2. Phys Rev Lett 1997, 78, 4063. 
(11) Togo, A.; Oba, F.; Tanaka, I. First-principles calculations of the ferroelastic transition between rutile-type and $\mathrm{CaCl}(2)$-type $\mathrm{SiO}(2)$ at high pressures. Phys Rev B 2008, 78, 134106.

(12) Ong, Z. Y.; Zhang, G. Efficient approach for modeling phonon transmission probability in nanoscale interfacial thermal transport. Phys Rev B 2015, 91, 174302.

(13) Ataca, C.; Sahin, H.; Ciraci, S. Stable, Single-Layer MX2 TransitionMetal Oxides and Dichalcogenides in a Honeycomb-Like Structure. Journal of Physical Chemistry C 2012, 116, 8983.

(14) Rasmussen, F. A.; Thygesen, K. S. Computational 2D Materials Database: Electronic Structure of Transition-Metal Dichalcogenides and Oxides. Journal of Physical Chemistry C 2015, 119, 13169.

(15) Kang, J.; Tongay, S.; Zhou, J.; Li, J. B.; Wu, J. Q. Band offsets and heterostructures of two-dimensional semiconductors. Appl Phys Lett 2013, 102, 012111.

(16) Williamson, I.; Hernandez, A. C.; Wong-Ng, W.; Li, L. High-throughput computational screening of electrical and phonon properties of two-dimensional transition metal dichalcogenides. JOM 2016.

(17) Sansonetti, J. E.; Martin, W. C.; July 1, 2009 ed.; National Institute of Standards and Technology: http://www.nist.gov/pml/data/handbook/, 2014.

(18) Shannon, R. D. Revised Effective Ionic-Radii and Systematic Studies of Interatomic Distances in Halides and Chalcogenides. Acta Crystallogr A 1976, 32,751 .

(19) Gu, X. K.; Yang, R. G. Phonon transport in single-layer transition metal dichalcogenides: A first-principles study. Appl Phys Lett 2014, 105, 131903.

(20) Swartz, E. T.; Pohl, R. O. Thermal-Boundary Resistance. Reviews of Modern Physics 1989, 61, 605. 
(21) Zhu, T. S.; Ertekin, E. Phonon transport on two-dimensional graphene/boron nitride superlattices. Phys Rev B 2014, 90, 195209. 


\section{CHAPTER EIGHT: CONCLUSIONS}

The research goal for this dissertation is to generate large data sets and analyze trends and relationships between structure, properties, and performance of skutterudite and 2D TMD compounds for electronic and energy applications. By understanding these design principles, we can tailor the structures to enhance and optimize material properties and performance. These materials are known to have good intrinsic electrical properties and tailorable structures making them excellent candidates for a number of energy and electrical applications. First-principles DFT calculations were employed to investigate skutterudites and 2D TMDs and how their electrical and phonon properties can be enhanced by two main structural changes: (1) doping and (2) the formation of a heterojunction.

Chapter One provides an overview of the goal and motivation for this dissertation. Four main objectives were used to achieve the goal including: (1) understanding dopant effects on skutterudite materials, (2) screening 2D TMD materials compositions, (3) understanding the effects of substitutional dopants in 2D TMDs, and (4) evaluating the impact of a lateral heterojunction between 2D TMDs. A summary of the dissertation, including the published works produced from this work, is also included in this chapter.

Chapter Two explains the background for each materials system investigated and highlights their applications and current state of research. Skutterudites are a widely studied class of materials because of their low-cost, easy processing, and intrinsic transport properties. Currently of interest for thermoelectric applications, their open, 
cage-like structures offer a unique opportunity for materials-by-design exploration. Much work has been done to investigate the effects of single-, double-, and multi-filled skutterudites which show promising enhancement in electrical and thermal properties. Substitutional doping has been shown to further enhance these properties, yet a fundamental investigation of fillers and substitutions is still required to fully optimize skutterudite compounds. TMDs also have large degrees of freedom in their structure including structure type, dimensionality, and composition. Single-layer, 2D TMD sheets have attracted interest as candidates for magnetics, electronics, optoelectronics, catalysis, and thermoelectric applications. Quantum confinement effects and diffusive boundary scattering can be exploited for improved electrical and transport properties. Smaller device features have shown promising enhancement of FETs. There remain three key areas for property improvement for these materials: identification of optimum compositions, substitutional doping, and heterostructure formation. A thorough investigation of these factors is necessary for further development of 2D TMD materials.

Chapter Three reviews the theoretical and computational methods employed in this dissertation. It explains how DFT is used to calculate the electronic and geometric ground state of a material through total energy minimization calculations. The resulting geometric, electrical, and phonon structures can be used to investigate the effects of dopants and heterostructures. Band energies can be expanded through the use of star functions to determine semi-classic transport coefficients while DFPT-based force constant calculations can be used to calculate phonon DoS, transmission coefficients across an interface, and thermal boundary conductance. 
Chapter Four investigates the structural, electrical, and thermal properties of two dopant types in skutterudite compounds: filler atoms and Sb-site substitution. Both the incorporation of $\mathrm{Ca} / \mathrm{Ce}$ filler atoms in $\mathrm{FeSb}_{3}$ and $\mathrm{Te} / \mathrm{Ge}$ substitution in $\mathrm{CoSb}_{3}$ decrease the lattice constant for the compounds. Te/Ge substitution leads to distortion in the pnictogen rings and a break in the cubic symmetry of the compound. Both dopants cause an increase in electrical conductivity through the reduction in electronic bandgap while Te/Ge substitution also causes a transition from direct to indirect semiconducting behavior for $x=3$. Phonon band broadening is observed in both doped systems and each are assumed to exhibit acoustic-mode dominated lattice thermal conductivity. Of the substitution concentrations, $x=3$ has the lowest acoustic phonon dispersion gradient implying that it experiences the lowest lattice thermal conductivity. This is consistent with experimental measurements. The presence of either dopant has shown enhancements in the thermoelectric properties of these skutterudite compounds. This computational study provided insight into experimental observations, advanced the understanding of dopant effects on skutterudite materials.

Chapter Five covers high throughput screening of 2D TMD compositions based on electrical and phonon properties. With the large number of potential compositions, focus was narrowed to three chalcogens $(\mathrm{S}, \mathrm{Se}, \mathrm{Te})$ and six transition metals $(\mathrm{Mo}, \mathrm{Ni}, \mathrm{Sc}$, Ti, V, and W) based on environmental implications, supply risk, and vulnerability to supply restrictions known as "criticality scores." These scores allowed for the exploration of compositional effects while focusing on materials that are better-suited for development. The physical features of constituent transition metals (e.g., atomic mass, atomic radius, and oxidation state) have significant impact on the overall properties of the 
2D TMD. For instance, as the metal mass increases, the $n$-type power factor, $S^{2} \sigma$, and phonon frequency gap increases. Also, increasing the radius causes an increase in 2D TMD layer thickness and Seebeck coefficient, $S$. The results of Chapter Five help identify principles for optimizing 2D TMD compositions for desired performance.

Chapter Six investigates the metal-site substitution effects on 2D TMD properties. These were analyzed with respect to their effect on structural, energetic, electrical, transport, and phonon properties. The overall dopant effect was found to decrease with concentration until they converge at 2.083 at.\% where the dopants are effectively isolated. Structural distortion, dopant binding energy, electron DoS, and phonon DoS confirm this convergence at 2.083 at.\%. Therefore, this concentration was used to investigate dopant type with a large number of different compositions corresponding to the formula, $\mathrm{M}^{\prime} \mathrm{M}_{15} \mathrm{X}_{32}\left(\mathrm{M}^{\prime}=\mathrm{Mo}, \mathrm{Ni}, \mathrm{Sc}, \mathrm{Ti}, \mathrm{V}\right.$, or $\mathrm{W} ; \mathrm{M}=\mathrm{Mo}$ or $\mathrm{W}$; and $\mathrm{X}=\mathrm{S}$, Se, or Te). Most structures exhibit minimal distortion upon doping except those doped with $\mathrm{Ni}$ or Sc. These are also the metals with the lowest oxidation states ( +2 and +3 , respectively, compared to the host metals' +6 ). Only the W-doped $\mathrm{MoX}_{2}$ and the Mo-doped $\mathrm{WX}_{2}$ compounds consistently exhibit semiconducting behavior and have the largest $p$-type $S^{2} \sigma$. Also, the presence of $\mathrm{W}$ and Mo dopants in the selenide and telluride compounds increase $\sigma$ by as much as $60 \%$ (at carrier concentration $N=10^{14}$ holes $/ \mathrm{cm}^{2}$ ). However, due to the reduction in $S$, the $S^{2} \sigma$ decreases for each doped system investigated. Phonon DoS results show acoustic region peak-broadening and narrower frequency gaps for Tidoped $\mathrm{MoS}_{2}$ suggesting a reduction in phonon lifetimes and increased acoustic-optical phonon scattering. A large amount of information on doping effects was discussed in this chapter which is useful for guiding the development of doped 2D TMD materials. 
Chapter Seven highlights the formation of a lateral heterostructure between 2D $\mathrm{MoS}_{2}$ and $2 \mathrm{D} \mathrm{MS} 2(\mathrm{M}=\mathrm{Cr}, \mathrm{Sc}, \mathrm{Ti}$, and $\mathrm{W})$. The structural, energetic, electronic, and phonon effects of this interface were investigated. The difference in oxidation state between the Mo and the M atoms plays a larger role in the heterostructure stability than the atomic mass or radius. The least stable systems - the ones having the highest formation energy and presence of negative (imaginary) phonon frequencies - involved Sc $(+3)$ and $\mathrm{Ti}(+4)$ which had the largest difference in oxidation state compared to Mo (+6). Alternatively, the $\mathrm{Cr}$ and $\mathrm{W}$, which both have +6 oxidation states, had low formation energies and no significant negative phonon frequencies suggesting mechanical stability. These were also the only systems to exhibit semiconducting behavior. The VBM and CBM for the heterostructures were found to be determined by the band alignment of the pure 2D TMD constituents. For the Cr system, the pure $\mathrm{CrS}_{2} \mathrm{VBM}$ is aligned with that of the pure $2 \mathrm{D} \mathrm{MoS}$ while the $\mathrm{CrS}_{2} \mathrm{CBM}$ is significantly lower. This causes the resulting bandgap of the heterostructure to be equivalent to the pure $\mathrm{CrS}_{2}$. The $\mathrm{W}$ system, however, forms a type II (staggered) alignment making the resulting heterostructure bandgap (1.59 $\mathrm{eV}$ ) smaller than the bandgap of either constituent (1.69 eV for $2 \mathrm{D} \mathrm{MoS}_{2}$ and $1.85 \mathrm{eV}$ for $2 \mathrm{D} \mathrm{WS}_{2}$ ). The phonon DoS results for the $\mathrm{W}$ system are also the only ones that exhibit a frequency gap between acoustic and optical phonon bands, suggesting that the $\mathrm{MoS}_{2}-\mathrm{WS}_{2}$ heterostructure experiences the lowest acoustic-optical phonon mode scattering among these heterostructures. The TBC of the $\mathrm{MoS}_{2}-\mathrm{WS}_{2}$ interface was found to be dominated by the acoustic phonons and limited by the difference in acoustic phonon velocities between the $\mathrm{MoS}_{2}$ and $\mathrm{WS}_{2}$. Our calculated TBC for this system is almost an order of 
magnitude smaller than that of the graphene $/ h-\mathrm{BN}$ heterostructure due to the reduced acoustic phonon velocities of the TMDs.

This dissertation offers an analysis of the structure-property relationship for a wide range of properties on bulk skutterudite and 2D TMD materials. An investigation of filler and substitutional dopant effects on skutterudite compounds provide insight to advance the understanding of electrical and phonon properties that experiments could not measure. This also offered a good benchmark material for developing a modeling scheme that was employed for 2D TMD materials. The three key outcomes of this work are: (1) a high throughput approach to compute and analyze electrical and phonon properties, (2) a screening method for investigating 2D TMD materials and highlighting preferred compositions, and (3) design principles for predicting structures and properties to guide experiments. The high throughput approach include: DFT-based total energy minimization calculations to investigate the geometric, energetic, and electronic structure data; Boltzmann transport theory, in combination with electronic band energies, to estimate $\sigma, S$, and $S^{2} \sigma$ values; DFPT-based second-order force constant calculations to determine phonon dispersion and DoS spectra; and the AGF method, using force constants as input, to compute interfacial heat flux, phonon transmission coefficients, and TBC. The input parameters and structures for each model were optimized prior to calculation. DFT-based input parameters include cutoff energies, $k$-point meshes, partialoccupancy smearing widths, and on-site DFT+U terms. For phonon calculations, supercell sizes were similarly determined through convergence tests. Literature values were used where available to compare resultant lattice constants, bond lengths/angles, bandgaps, and phonon dispersion data. This approach mitigated errors in the model, 
which can now be used to analyze electrical and phonon properties of similar systems, in a fraction of the time. Due to this work, dozens of 2D TMD structures can be analyzed within days, whereas initial optimization calculations for each structure took up to one week to compute. The design principles determined in this dissertation compare known literature values for common oxidation states, atomic masses, and atomic radii of constituent transition metal atoms to the properties of resulting 2D TMD compounds. These principles include:

- Metal-site dopant stability is dependent on matching atomic radii between the dopant and solvent metal

- Dopants can increase electrical conductivity in TMD selenides and tellurides but decrease $S^{2} \sigma$

- The choice of similar oxidation states between transition metals in adjacent TMDs is crucial for lateral heterostructure stability.

Future work includes a broader investigation of 2D TMD heterostructures and the effects of structure, composition, and transition metal physical features on stability, electrical, and phonon properties. The primary areas of interest for this investigation include the effects of: (i) structure type (i.e., 2H- vs. 1T-type), (ii) composition including choice of transition metal and chalcogen, (iii) heterostructure interface between TMDs and other 2D structures such as graphene, and (iv) alternate heterostructure interfaces such as vertical 2D TMD stacking. Further property improvements can be achieved by combining these structures with substitutional doping. Overall, these materials offer great potential for materials-by-design exploration and understanding their 
structural, electrical, and phonon properties are essential for advancement towards commercial applications. 